\title{
The Regulated Expression of Focal Adhesion Kinase (FAK) Family Kinases Modulates Mononuclear Phagocyte Differentiation, Migration, and Breast Tumor Progression
}

\author{
Michael F. Gutknecht \\ Plainwell, Michigan \\ B.S. Duke University 1997 \\ M.S. Georgetown University 2002
}

A Dissertation presented to the Graduate Faculty

of the University of Virginia in Candidacy for the Degree of

Doctor of Philosophy

Department of Microbiology, Immunology, and Cancer Biology

University of Virginia

November, 2013

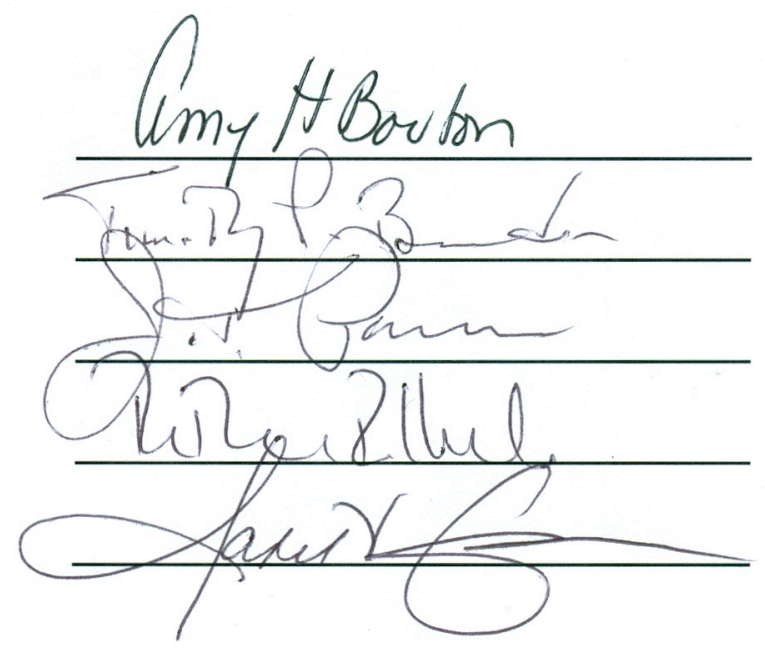




\section{Abstract}

A hallmark of all unicellular and multicellular organisms is the maintenance of homeostasis, which is achieved through the interpretation of extracellular stimuli coordinated with the appropriate effector response to these cues. This quality is exquisitely evident in cells of the mononuclear phagocyte system (MPS). Sharing lineage with primitive phagocytic cells and amoebocytes that reside in invertebrates, the MPS has surfaced in more evolved organisms to populate every tissue. The MPS is tightly regulated, which is reflected in the heterogeneity of cell populations that comprise this system and the effector capabilities that these cells harbor. Evidence indicates that, during steady state and disease conditions, the essential characteristics of phenotypic plasticity and migration associated with to these cells are utilized to maintain balance as well as potentiate pathologies.

Intracellular signaling cascades have evolved to link the receptors that sense the microenvironment with intrinsic cell properties that permit effector function. My thesis research has focused on the role of the related non-tyrosine kinases focal adhesion kinase (FAK) and proline rich tyrosine kinase $2(\mathrm{Pyk} 2)$ in the regulation of MPS development and function. Data from these studies have revealed that expression of FAK and Pyk2 are regulated during MPS differentiation, and that Pyk2 is expressed prior to FAK as lineage-committed monocytic cells differentiate. This is evident during the generation of monocyte cell populations in response to differentiation factors, where FAK can be seen to enhance monocyte differentiation and/or survival while Pyk2 blocks differentiation and/or enhances survival under certain conditions. This work has implications for a wide array of biological processes, including hematopoiesis in the bone marrow, the maintenance of circulating Ly6 $\mathrm{C}^{\text {high }}$ and $\mathrm{Ly} 6 \mathrm{C}^{\mathrm{low}}$ monocyte populations, and the generation of MPS populations in peripheral tissues during steady state and disease. 
Prior studies have shown that FAK regulates the migration of fully differentiated macrophages toward chemokines and growth factors in vitro. In addition, FAK is necessary to generate macrophage accumulation in the peritoneal cavity during inflammation. We demonstrate here that the regulated expression of FAK in monocytic cells has functional implications. Our work revealed that FAK is nearly undetectable in Ly6 $\mathrm{C}^{\text {high }}$ monocytes but that expression increases significantly as monocytes differentiate and downregulate Ly6C. Utilizing monocytes derived from wild type (WT) and conditional FAK knockout mice $\left(\mathrm{FAK}^{\Delta \text { myeloid }}\right)$, we demonstrated that Ly6C ${ }^{\text {low }}$ monocytes lacking FAK migrated less efficiently toward macrophage colony stimulating factor (M-CSF) than those from WT mice. Additionally, Ly6 $\mathrm{C}^{\text {high }}$ monocytes displayed reduced migration relative to Ly6 $\mathrm{C}^{\text {low }}$ monocytes, and the Ly6 $\mathrm{C}^{\text {high }}$ populations derived from $\mathrm{WT}$ and $\mathrm{FAK}^{\Delta \text { myeloid }}$ migrated equivalently. This suggests that migratory properties associated with monocytes are enhanced in congruence with FAK expression, and that efficient migration of these cells is FAK-dependent.

Evidence from human cancer patients and murine tumor models indicate that mononuclear phagocytes localize to the tumor microenvironment and influence tumor progression. We assessed if FAK expression in monocyte populations may also regulate progression of the MMTV-PyVmT murine breast carcinoma model. The rate of early stage tumor outgrowth was enhanced in $\mathrm{FAK}^{\Delta \text { myeloid }}$ mice crossed onto this background relative to WT MMTV-PyVmT mice, indicating either that FAK in myeloid lineage cells is necessary for an efficient anti-tumor response and/or that it blocks pro-tumor functions of these cells. Our evidence indicates that this regulation is limited to early stage events, as tumor vasculature and metastatic tumor burden are similar in both cohorts. We are currently investigating whether the migratory or functional capacity of tumor associated Ly6C ${ }^{\text {low }}$ monocytes is involved in orchestrating MPS control of early tumor outgrowth. 


\section{Acknowledgements}

Given that the day I hand in this thesis happens to coincide with Veterans Day, I think it presents an opportunity to begin my acknowledgments by thanking the Department of Defense (DoD) for funding a significant portion of my graduate work. The DoD pre-doctoral breast cancer training fellowship has not only supported me but gave me the opportunity to visit with breast cancer patients during the Era of Hope conference. This was an impactful event and added significance to what we all do in the lab. I am also grateful for funding received through the NIH cancer training grant and the Wagner fellowship, which in addition to financial support provided a boost in confidence during the early years of my study. Additionally, I would like to acknowledge the University of Virginia as a whole, and the people associated with the institution and community. This is a special place.

My tenure as a graduate student has made me more aware and appreciative of what goes into the job of mentor and principle investigator. From my perspective, an essential quality that must be maintained every day is a relentless approach to life in the lab. Amy has served as a model in this regard, and I attempt to incorporate this trait into the way I practice science. Her diligence comes through at different moments, ranging for data analysis to keeping the next experiment on track with the big picture. I feel this may be most evident in her writing, an area in which I have benefitted greatly due to her guidance. Perhaps what I may be most thankful for is her ability to bring out the best attributes in people. I think she fosters this and recognizes the positive characteristics that each of us in lab brings to the table. Thank you Amy.

On that note, the members of the Bouton lab have had a tremendous impact on my science and on

my life. I think we all appreciate that we are in this together and work our best to help make 
success achievable. Every day is revealing and provides an opportunity to learn. Thank you Bouton Lab.

I also want to thank my committee, Drs. Bullock, Bender, Cross, and Parsons, who have without question improved my data analysis and methodology of conducting experiments. Their support of my project at times when it was needed will forever be recognized and appreciated.

As graduate students, we are expected to be proficient in numerous disciplines that allow us to complete our experiments. When expertise is needed, we are surrounded by people in the core facilities that provide essential support. They cannot be given ample recognition for what they do.

I also want to recognize Sandy Weirich, who provides daily support to all graduate students in the department yet maintains the ability to connect with individuals and listen. She always finds a way to come through with the right information at the right time.

This could not have been possible without the support of my parents and sister. Even though we are spread out around this country, I always know that my back is covered. The same is true for Pono. 


\section{Table of Contents}

Preamble..... 1

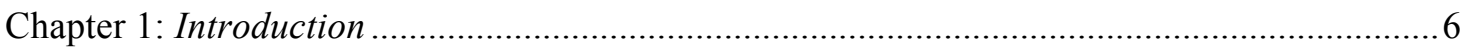

1.1 Perspective on the mononuclear phagocyte system ................................................ 6

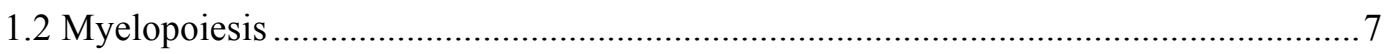

1.3 Differentiation of progenitor cells to committed monocytes ........................................ 10

1.3.1 Regulated expression of surface antigens................................................... 10

1.3.2 Establishment of two distinct monocyte populations ....................................13

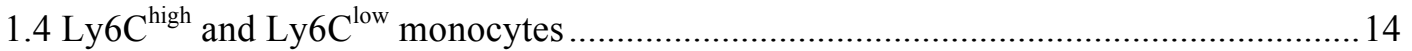

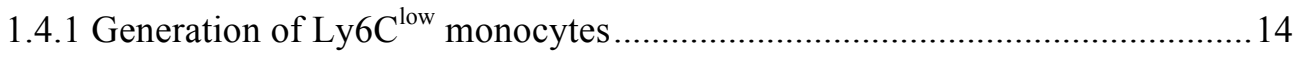

1.4.2 Ly6 $\mathrm{C}^{\text {high }}$ monocyte trafficking to peripheral sites .........................................17

1.4.3 Ly6 $\mathrm{C}^{\text {low }}$ monocyte function in the vasculature ............................................... 18

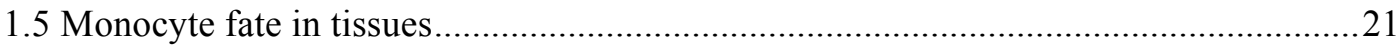

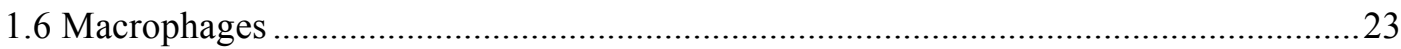

1.6.1 Derivation of tissue resident macrophages ...............................................23

1.6.2 Regulation of MPS populations by colony stimulating factors .......................24

1.6.3 Coordinated effect of multiple stimuli on the MPS.......................................26

1.7 Perspective on mononuclear phagocytes and tumors ..............................................27

1.8 Tumor associated macrophages (TAM) and myeloid derived suppressor cells (MDSC)

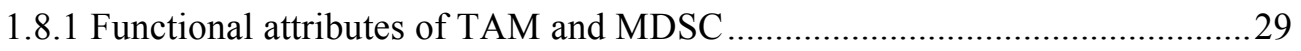

1.8.2 Characterization of tumor associated monocyte populations ..........................32

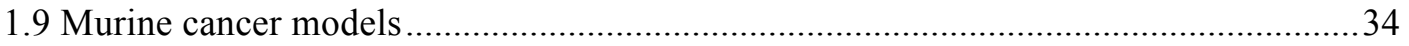

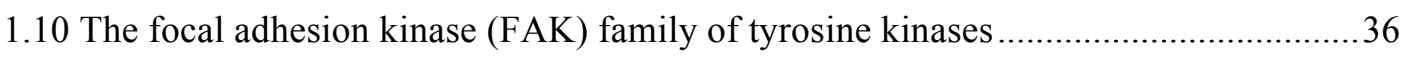

1.10.1 Identification and characterization of focal adhesion kinase (FAK) ..............36

1.10.2 Identification and characterization of proline rich tyrosine kinase 2 (Pyk2)37 


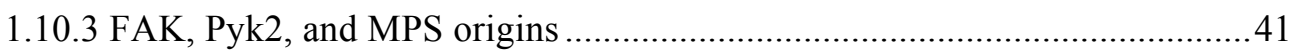

1.10.4 Functional relationship of FAK and Pyk2 .............................................. 41

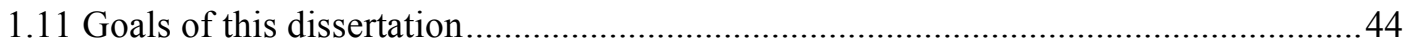

Chapter 2: The regulated expression of FAK and Pyk2 modulates monocyte differentiation .....49

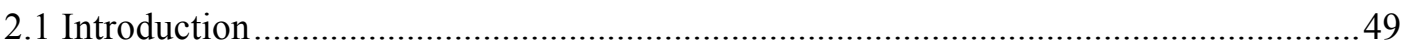

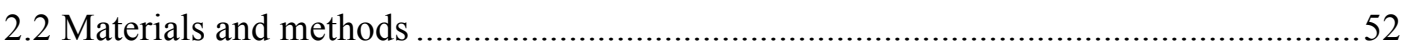

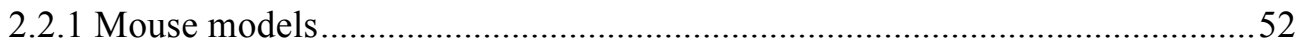

2.2.2 Harvest and preparation of cell suspensions from mouse tissues....................52

2.2.3 Flow cytometry to distinguish $\operatorname{Lin}^{-} \mathrm{CD} 11 \mathrm{~b}^{+}$monocyte populations.................52

2.2.4 Immnuomagnetic column separation of cell populations ...............................53

2.2.5 In vitro derived monocyte/macrophage populations from BM ......................54

2.2.6 Separation of Ly6C $\mathrm{C}^{\text {high }}$ and Ly6 $\mathrm{C}^{\text {low }}$ monocyte populations ...........................54

2.2.7 Immunoblot detection of FAK and Pyk2 ….............................................55

2.2.8 In vitro differentiation of FACS isolated Ly6 $\mathrm{C}^{\text {high }} \mathrm{BM}$ monocytes .................55

2.2.9 Isolation of in vitro derived monocyte populations by FACS........................56

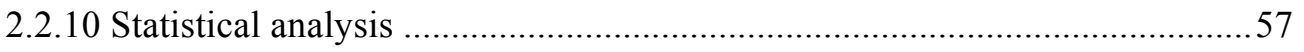

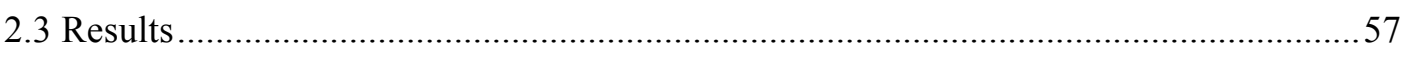

2.3.1 FAK and Pyk2 protein expression is elevated in more differentiated $\mathrm{CD} 11 \mathrm{~b}^{+}$

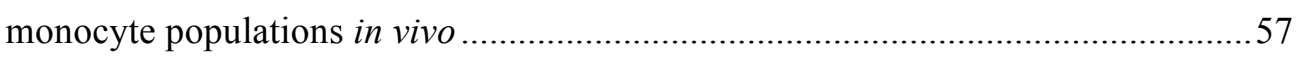

2.3.2 Pyk2 is required for the maintenance of $\mathrm{Ly} 6 \mathrm{C}^{+} \mathrm{F} 4 / 80^{+}$mononuclear phagocytes

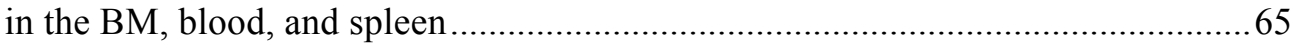

2.3.3 FAK is expressed predominantly in Ly6 $\mathrm{C}^{\mathrm{low}}$ monocytes ...............................70

2.3.4 FAK expression increases with the loss of Ly6C and Pyk2 expression increases

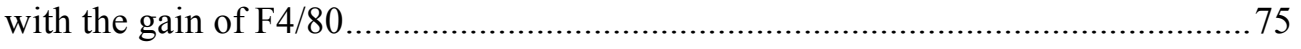

2.3.5 FAK and Pyk2 regulate monocyte differentiation in response to GM-CSF + IL-

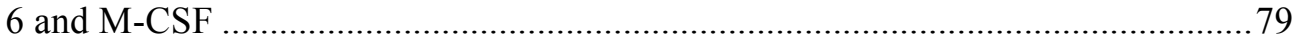

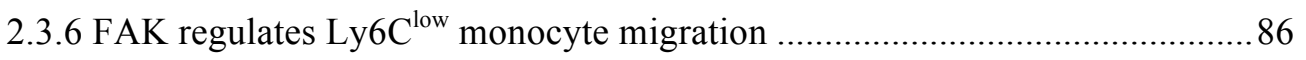

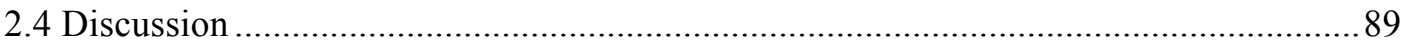


Chapter 3: The regulation of murine breast tumor outgrowth and mononuclear phagocyte

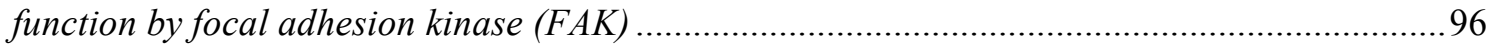

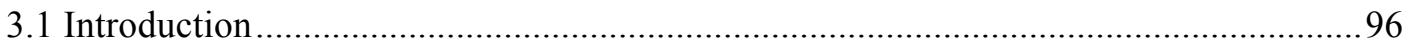

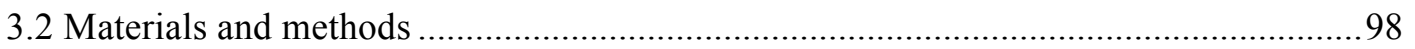

3.2.1 Generation of mouse models .....................................................................98

3.2.2 Measurement of MMTV-PyVmT primary tumor outgrowth ..........................101

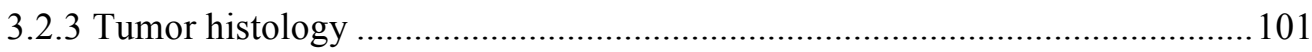

3.2.4 Quantification of vessel density in day 105 tumor specimens .......................103

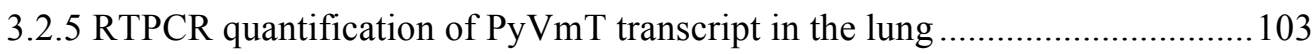

3.2.6 Determination of lung metastatic burden by histology ..................................103

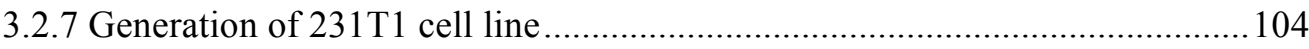

3.2.8 In vitro induction of macrophage polarization ................................................ 104

3.2.9 Analysis of BMDM for pro-MMP9 expression ...........................................105

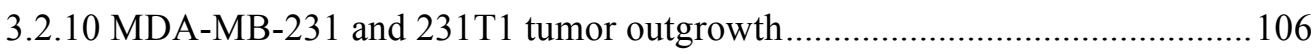

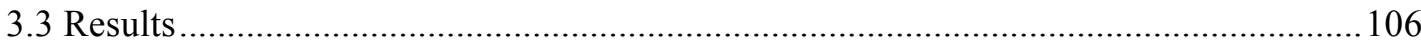

3.3.1 FAK expression in myeloid lineage cells regulates early stages of tumor initiation/growth but not later stage growth or vascularity ....................................106

3.3.2 FAK does not regulate macrophage behavior critical for lung metastasis ......116

3.3.3 FAK does not regulate macrophage polarization in response to classical Th1

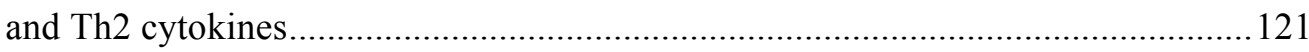

3.3.4 FAK regulates the compartmentalization of pro-MMP9...............................122

3.3.5 FAK regulates tumor growth in the MDA-MB-231 orthotopic tumor model.130

3.4 Discussion

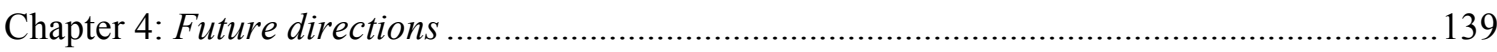

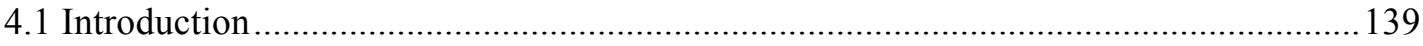

4.2 What mononuclear phagocyte populations express FAK and Pyk2? ..........................139 
4.3 How does FAK coordinate with other molecules that regulate differentiation of Ly6C ${ }^{\text {high }}$

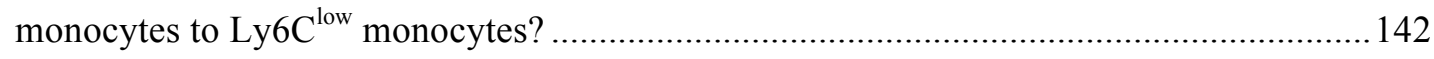

4.4 What is the role of FAK and Pyk2 in the effector functions of mononuclear phagocyte

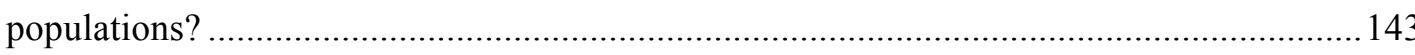

4.5 Perspective on FAK and Pyk2 in mononuclear phagocytes ....................................... 152

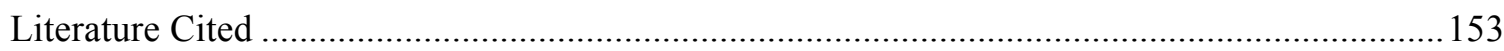




\section{Index of Figures}

\section{Chapter 1}

Figure 1.1: FAK expression in myeloid lineage cell populations is not equivalent ............5

Figure 1.2: Myeloid lineage commitment in the bone marrow..........................................

Figure 1.3: Monocyte differentiation occurs independently in the BM and blood..............12

Figure 1.4: Surface antigens distinguish Ly6C $\mathrm{C}^{\text {high }}$ and Ly6C $\mathrm{C}^{\text {low }}$ monocyte populations .......16

Figure 1.5: Ly6 $\mathrm{C}^{\text {high }}$ and Ly6 $\mathrm{C}^{\text {low }}$ monocytes utilize different mechanisms for attachment and trafficking on the endothelium

Figure 1.6: The non-receptor tyrosine kinases FAK and Pyk2 display a high degree of sequence homology and mediate signaling through cell surface receptors ..... .39

Figure 1.7: Potential FAK regulated interactions between the primary tumor microenvironment and macrophages 48

\section{Chapter 2}

Figure 2.1: Isolation of $\mathrm{CD} 1 \mathrm{bb}^{+}$monocytes from murine bone marrow by immunomagnetic column separation .59

Figure 2.2: FAK and Pyk2 protein expression is regulated in in BM, blood, and splenic monocytes

Figure 2.3: Identification of $\operatorname{Lin}^{-} \mathrm{CD} 11 \mathrm{~b}^{+}$monocytes in BM, blood, and spleen by flow cytometry

Figure 2.4: Loss of Pyk2 results in reduced proportion of Ly $6 \mathrm{C}^{+} \mathrm{F} 4 / 80^{+}$monocytes in blood and spleen.

Figure 2.5: Ly6C ${ }^{\text {high }}$ monocytes in the BM display equivalent levels of BrdU incorporation

Figure 2.6: FAK and Pyk2 expression increase during monocyte differentiation in vitro.

FAK expression is elevated in monocytes that have reduced Ly6C surface expression ......72 Figure 2.7: FAK expression is elevated in Ly6 $\mathrm{C}^{\text {low }}$ monocytes derived from M-CSF treated $\mathrm{BM}$ 
Figure 2.8: Ly6C $\mathrm{C}^{\text {high }} \mathrm{BM}$ monocytes differentiate into $\mathrm{Ly}^{6} \mathrm{C}^{+} \mathrm{F} 4 / 80^{+}$and Ly6C-F4/80 monocytes. FAK and Pyk2 expression are regulated during monocyte differentiation .......77

Figure 2.9: FAK and Pyk2 regulate monocyte differentiation ........................................... 81

Figure 2.10: BrdU incorporation is equivalent in Ly6 $\mathrm{C}^{+}$monocytes derived GM-CSF + IL-6

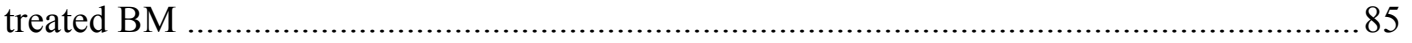

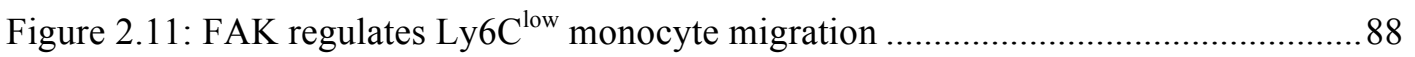

Figure 2.12: Potential mechanisms of FAK and Pyk2 regulation of monocyte differentiation,

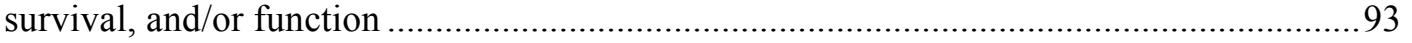

\section{Chapter 3}

Figure 3.1: Genetic recombination at the FAK locus and deletion of FAK protein in myeloid lineage cells from $\mathrm{FAK}^{\Delta \text { myeloid }}$ mice 100

Figure 3.2: Tumor initiation in the MMTV-PyVmT model is not regulated by FAK in myeloid lineage cells. Initiation of tumor growth in mammary glands 2-4 (MG 2-4) is significantly delayed relative to MG1 108

Figure 3.3: MG1 tumors at day 91 are significantly larger in $\mathrm{FAK}^{\Delta \text { myeloid }}$ mice relative to WT mice and display characteristics indicative of mammary intraepithelial neoplasia (MIN)/early carcinoma tumor stage.

Figure 3.4: MG1 tumor burden at day 105 is significantly greater in $\mathrm{FAK}^{\Delta \text { myeloid }}$ mice relative to WT mice and displays hallmark features of advanced disease......

Figure 3.5: Vessel density is equivalent in day $105 \mathrm{MG} 1$ tumors from WT and FAK $\mathrm{K}^{\Delta \text { myeloid }}$

mice.

Figure 3.6: Metastatic tumor burden is equivalent in $\mathrm{WT}$ and $\mathrm{FAK}^{\Delta \text { myeloid }}$ mice

Figure 3.7: PyVmT transcript levels in the lungs of day $105 \mathrm{WT}$ and $\mathrm{FAK}^{\Delta \text { myeloid }}$ mice are equivalent

Figure 3.8: Macrophage polarization to IFN- $\gamma+$ LPS is not regulated by FAK

Figure 3.9: FAK does not regulate polarization to the M2 activation state in response to IL-4 treatment 126 
Figure 3.10: FAK regulates compartmentalization of pro-MMP9 in BMDM 129

Figure 3.11: Tumorigenesis is enhanced with $231 \mathrm{~T} 1$ cells compared to parental MDA-MB231 cells 132

Figure 3.12: Tumor doubling time is greater in $\mathrm{FAK}^{\Delta \text { myeloid }}$ mice relative to WT mice ....... 134

\section{Chapter 4}

Figure 4.1: Potential FAK-dependent mechanisms utilized by tumor associated mononuclear phagocytes to regulate primary tumor outgrowth...... 150 


\section{Index of Abbreviations}

ATCC: American Type Culture Collection

BM: bone marrow

BMDM: bone marrow derived macrophage

BSA: bovine serum albumin

CD: cluster of differentiation

CFKO: Cre recombinase induced FAK knockout

cMoP: common monocyte progenitor

CM: conditioned media

CMP: common myeloid progenitor

Cre: cre recombinase

CSF-1: colony stimulating factor 1

DC: dendritic cell

EC: endothelial cell

ECM: extracellular matrix

EGF: epidermal growth factor

EGFP: enhanced green fluorescent protein

ER: estrogen receptor

ERK: extra cellular regulated kinase

FACS: fluorescence-activated cell sorting

FAK: focal adhesion kinase

FBS: fetal bovine serum

FKN: fractalkine 
FMO: fluorescence minus one

FN: fibronectin

GAPDH: glyceraldehyde phosphate dehydrogenase

G-CSF: granulocyte colony stimulating factor

GFP: green fluorescent protein

GM-CSF: granulocyte/macrophage colony stimulating factor

GMP: granulocyte/macrophage progenitor

gp130: glycoprotein 130

G-MDSC: granulocytic myeloid derived suppressor cell

GPI: glycosyl phosphatidyl inositol

Grb2: growth factor receptor binding protein 2

HBSS: Hanks balanced salt solution

HIF-1 $\alpha$ : hypoxia inducible factor $1 \alpha$

HLA: human leukocyte antigen

HSC: hematopoietic stem cell

IFN- $\gamma$ : interferon gamma

IHC: immunohistochemistry

IL: interleukin

iMacs: inhibitory macrophages

ImC: immature cells

JAK: janus kinase

JNK: c-Jun $\mathrm{N}$ terminal kinase

LFA: lymphocyte function associated antigen

Lin: lineage 
LPS: lipopolysaccharide

LTR: long terminal repeat

Ly: lymphocyte antigen

LysM: lysozyme M

MACS: magnetic-activated cell sorting

MAPK: mitogen activated protein kinase

MCP-1: monocyte chemoattractant protein-1

M-CSF: macrophage colony stimulating factor

M-CSFR: macrophage colony stimulating factor receptor

MDA-MB-231: breast cancer cell line

MDP: monocyte dendritic cell progenitor

MDSC: myeloid derived suppressor cell

MEP: megakaryocyte/erythrocyte progenitor

MG: mammary gland

MHC: major histocompatibility complex

MIN: mammary intraepithelial neoplasia

MIP-1 $\alpha$ : macrophage inflammatory protein $1 \alpha$

M-MDSC: monocytic-myeloid derived suppressor cell

MMP: matrix metalloproteinase

MMTV: mouse mammary tumor virus

MPS: mononuclear phagocyte system

NIH: National Institutes of Health

NK: natural killer

NO: nitric oxide 
PBS: phosphate buffered saline

PCR: polymerase chain reaction

PDGF: platelet derived growth factor

PI: protease inhibitors

PI3K: phosphatidyl-inositol 3 kinase

PKC: protein kinase $\mathrm{C}$

PR: progesterone receptor

Pyk2: proline rich tyrosine kinase 2

PyVmT: polyoma virus middle $\mathrm{T}$ antigen

RAFTK: related adhesion focal tyrosine kinase

RES: reticuloendothelial system

RSMC: rat smooth muscle cells

ROS: reactive oxygen species

RT: room temperature

SCF: stem cell factor

SCID: severe combined immunodeficiency

SDF-1 $\alpha$ : stromal cell-derived factor-1 alpha

SH: Src homology

STAT: signal transducer and activator of transcription

TAM: tumor associated macrophage

TED: transepithelial dendrites

TEM: Tie2-expressing monocytes

Th: T helper lymphocyte

TLR: Toll like receptor 
TMEM: tumor microenvironment of metastasis

TNF- $\alpha$ : tumor necrosis factor $\alpha$

UVA: University of Virginia

VEGF: vascular endothelial growth factor

VEGFR1: vascular endothelial growth factor receptor -1

VLA: very late antigen

WT: wild type 


\section{Preamble}

Innate immunity is a conserved biological process present throughout the plant and animal kingdoms (Hoffmann et al., 1999). Cells that comprise the innate immune system harbor the requisite tools for direct interaction with target cells as well as to mediate adaptive immune responses (Galli et al., 2011). Macrophages have long been front and center as one of the major components of the innate immune system due to their ability to coordinate directional movement with the detection and elimination of foreign entities (Gordon, 2007). However, more recent work has shown that macrophages can also mediate additional outcomes that reflect the full complement of their activity: tissue repair and remodeling, inflammation, the resolution of inflammation, homeostasis, and disease progression (Gordon and Taylor, 2005; Wynn et al., 2013; Murray and Wynn, 2011; Pollard, 2009).

My research has focused on terminally differentiated macrophages and the precursor cells from which they derive. It was initiated at a time when research in our lab intersected with new knowledge about how macrophages and tumors interact. Reports from Mantovani and colleagues illuminated macrophage plasticity in the context of tumors and provided the foundation for the M1 and M2 tumor associated macrophage polarization states that dominated the literature (Martinez et al., 2006; Mantovani et al., 2005). The data showed that macrophages could acquire a gene expression profile consistent with an anti-tumor effector function. These M1, or inflammatory, macrophages were opposed by the tumor-promoting attributes of M2, or alternatively activated, macrophages. This subset promoted angiogenesis and was characterized as having a wound healing phenotype. The dichotomy was not unique to a tumor setting, as the maintenance of homeostasis relied on the activities of both subsets. Research has since softened this rigid distinction; macrophages are now viewed as having the capability of falling within all 
regions of the activation state spectrum (Mosser and Edwards, 2008). The appreciation that this cell lineage possesses heightened phenotypic plasticity has clearly been strengthened during this period. Near the same time, research from the Condeelis and Pollard groups indicated that the interactions of tumor cells and macrophages were intimate and critical for metastasis (Wyckoff et al., 2007). Perhaps their greatest contribution to the field were live images of these associations in real time and the elucidation of communication networks between the two cell types (Sidani et al., 2006). Their data showed that tumor cells co-opted macrophage behavior to leave the primary microenvironment and metastasize.

Concurrently in our lab, then graduate student Kate Owen was finalizing a manuscript that showed focal adhesion kinase (FAK) regulated macrophage cytoskeletal dynamics and migration toward chemokines, cytokines, and growth factors (Owen et al., 2007). Accumulation of macrophages to sites of inflammation was also compromised with the loss of FAK. Her results had two important broad implications. First, FAK regulated macrophage migration in response to a variety of factors and was shown to be relevant in vivo. These results were consistent with FAK functions in other cell types. Second, her work substantiated a role for FAK in mononuclear phagocytes. Placing these results in context, much of the field at the time was focused on the activity of proline rich tyrosine kinase 2 (Pyk2), a FAK family member that was known to be expressed predominantly in cells of the nervous and hematopoietic systems. A manuscript published a few years earlier had addressed the role of Pyk2 in the regulation of macrophage function, but little was known about FAK function in these cells (Okigaki et al., 2003). Additionally, discrepancies existed in the literature regarding FAK expression in macrophages. In fact, five manuscripts published between 1994 and 2008 reported that monocytes and/or macrophages did not express FAK (Lin et al., 1994; De Nichilo and Yamada, 1996; Li et al., 1998; Kedzierska et al., 2001; Park et al., 2008). The work by our lab unequivocally placed FAK on the macrophage map. 
To complete her studies, Dr. Owen developed a mouse model in which FAK was conditionally deleted in cells of the myeloid lineage, which includes all monocytic cells. This provided a means to determine if FAK played a role in monocyte-mediated tumor progression. Synergizing the ongoing research in the tumor associated macrophage field with our results, we proposed the hypothesis that FAK regulates critical aspects of macrophage behavior that support tumor progression and metastasis. In collaboration with Dan Hershey, we crossed the conditional FAK knockout mice onto an immunodeficeint background, which permitted orthotopic transplantation of human breast carcinoma cells, and with a strain that was genetically engineered to develop spontaneous mammary tumors. As the tumor studies were ongoing, it became clear that FAK expression in different myeloid cell populations was not equivalent, and that FAK was elevated in differentiated macrophages relative to less differentiated monocytes (Figure 1.1). The literature was suggestive of this finding, and subsequent experiments during the course of my work confirmed this to be the case (Glodek et al., 2007; Kume et al. 1997; Owen et al. 2007). In Chapter 2, I will present research that addresses how the regulated expression of FAK and Pyk2 modulates monocyte differentiation. Chapter 3 of this thesis will provide a summary of the experiments addressing the role of FAK in regulating tumor progression. 
Figure 1.1 FAK expression in myeloid lineage cell populations is not equivalent. Myeloid lineage cells from WT and $\mathrm{FAK}^{\Delta \text { myeloid }}$ (conditional FAK knockout) mice were derived using several techniques. $\mathrm{CD} 11 \mathrm{~b}^{+}$cells from the bone marrow (BM) were isolated by immunomagnetic column separation. Whole BM was cultured in the presence of GM-CSF [40ng/ml] + IL-6 [40ng/ml], GM-CSF [40ng/ml], or L929 conditioned media containing M-CSF for four days to promote monocyte and/or granulocyte differentiation. (A) Cell lysates from the 8 cell populations were normalized for total protein concentration by BCA method and resolved by SDS/PAGE. FAK expression was determined by immunoblot. ERK serves as the loading control. (B) Cytospin preparations from WT CD11 b ${ }^{+}$BM, GM-CSF + IL-6, GM-CSF, and M-CSF cell suspensions were stained with hematoxylin and eosin. Note the small, mixed myeloid population in the BM compared to the uniform morphology and large size of M-CSF treated cells. 
A.

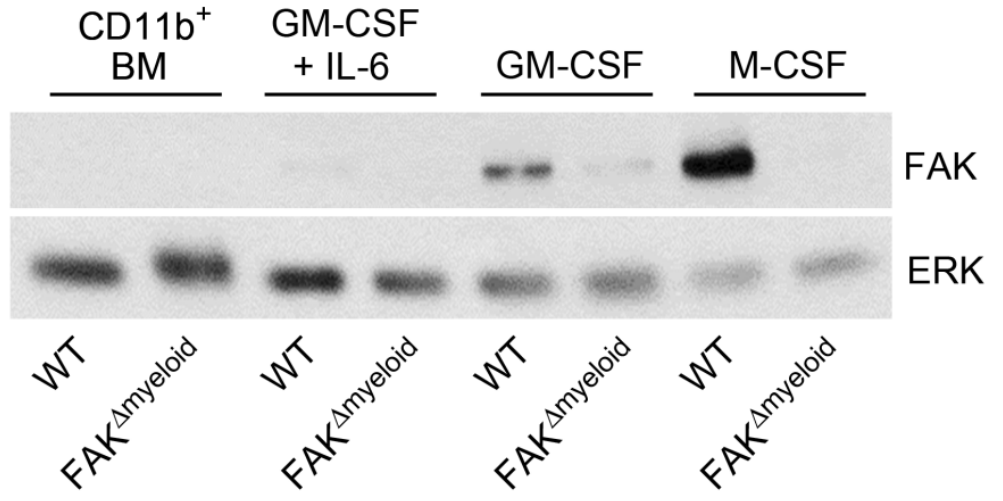

B.

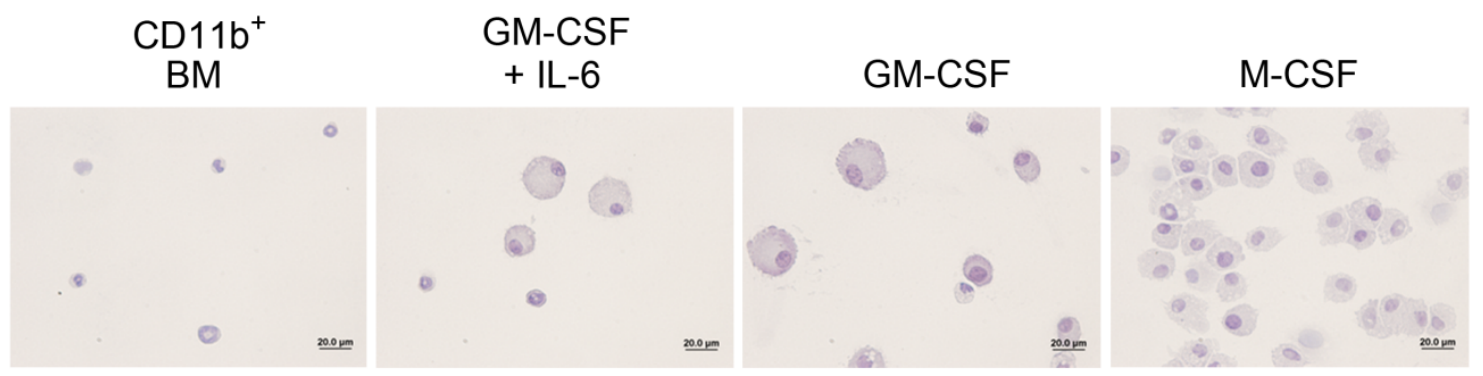




\section{Chapter 1}

Introduction

\subsection{Perspective on the mononuclear phagocyte system}

The mononuclear phagocyte system (MPS) is a heterogeneous population of monocyte lineagecommitted cells. Consisting of monocyte progenitors in the bone marrow, monocytes in circulation, and terminally differentiated macrophages at peripheral sites, mononuclear phagocytes represent a continuum of differentiation and maturation coordinated with precise trafficking (Hume 2006; Hume 2008; Hume et al. 2002). The MPS is a rarity in basic cell biology and tissue structure, as monocytic cells originate anatomically a great distance from where they carry out effector function (a quality they share with other hematopoietic cells) and they persist in steady state as both adherent and non-adherent cells. Consequently, extensive conceptual refinement has occurred to connect tissue resident phagocytes with circulating precursor monocytes. Discoveries in the late 1800 s by Metchnikoff of phagocytic cells in starfish and the water-flea, and the less celebrated work of others, provided the foundation for what we regard today as tissue macrophages (Modlin and Cheng, 2004; Gordon, 2008). The proposal of the reticuloendothelial system (RES) by Aschoff in the 1920s, which viewed histiocytes (tissue macrophages) and reticuloendothelia (phagocytic endothelia) as one system of local origin, was later modified by Van Furth and colleagues in the 1960s through pioneering work on circulating monocyte and macrophage kinetics (Takahashi, 2000; Van Furth and Cohn, 1968). Additional studies in the interim helped to more firmly establish a monocyte progenitor-to-maturemacrophage relationship during the progression from bone marrow to blood to peripheral tissues (Takahashi, 2000). Recent advances utilizing molecular genetics and reporter mice that permit fate mapping of monocyte lineage cells have substantiated the MPS and placed it in context with 
tissue macrophage populations established during development (Chow et al., 2011). What has emerged is a theme that cells of the MPS are highly responsive to external stimuli and migratory in nature.

\subsection{Myelopoiesis}

Monocytes and macrophages are members of the myeloid lineage of blood cells, which also consists of neutrophils, dendritic cells (DC), and osteoclasts (Iwasaki et al., 2007). The origins of this lineage lie predominantly in the bone marrow (BM), where the identification of myeloid progenitor populations has occurred through the isolation of murine cells that express a defined repertoire of surface antigens and the response of these cell populations to colony stimulating factors (CSF) ex vivo (Metcalf, 1989). It is thought that initial decisions of lineage commitment are stochastic, involving the antagonism and coordination of transcription factors present in hematopoietic stem cells (HSC) followed by the proliferation and survival of progressively more committed progenitor cell populations in response to specific growth factors and cytokines that drive lineage specificity (Enver et al., 2009; Orkin and Zon, 2008; Valledor et al., 1998; Lenny et al., 1997). Identification of the common myeloid progenitor (CMP) and the monocyte dendritic cell progenitor (MDP) signaled two key advancements (Akashi et al., 2000; Fogg et al., 2006). Isolation of the CMP distinguished granulocyte/macrophage progenitors (GMP) and megakaryocyte/erythrocyte progenitors (MEP) from cells that were committed to becoming lymphocytes. The subsequent purification of the more restricted MDP population segregated the GMP and placed monocyte and dendritic cell precursors in parallel with granulocyte progenitors. The transcription factors and growth factors that regulate the formation and persistence of these populations are summarized in Figure 1.2. 
Figure 1.2 Myeloid lineage commitment in the bone marrow. Schematic illustrates the progression of myeloid lineage commitment from hematopoietic stem cell (HSC) to common myeloid progenitor (CMP) to granulocyte-macrophage progenitor (GMP) to macrophagedendritic cell progenitor (MDP). The growth factors and transcription factors regulating each stage of progression are listed below the cell populations. Cells of the lymphoid lineage, erythrocytes and megakaryocytes, and granulocytes are excluded as cells commit to MDP. 


Bone Marrow

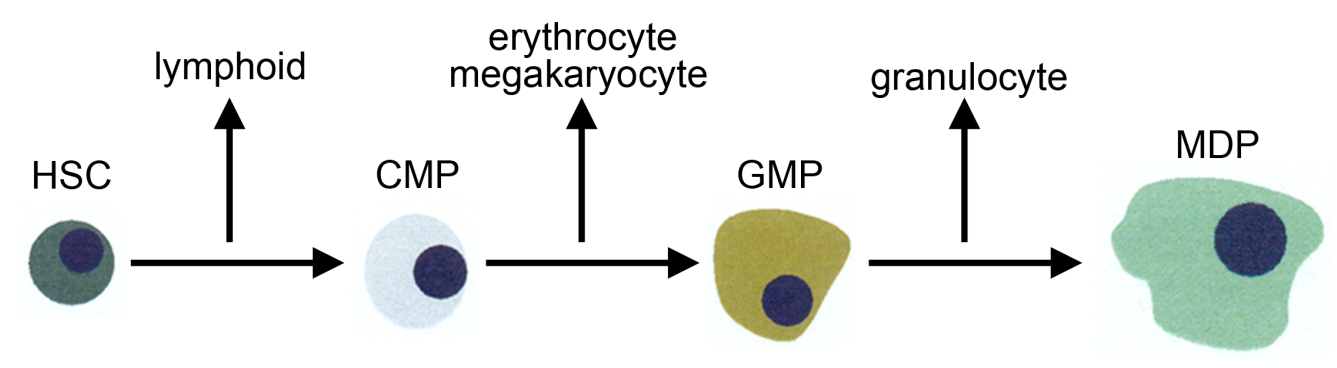

\begin{tabular}{|c|c|c|c|}
\hline $\begin{array}{l}\text { growth } \\
\text { factors }\end{array}$ & $\begin{array}{l}\text { IL-1 } \\
\text { IL-6 } \\
\text { IL-3 }\end{array}$ & $\begin{array}{l}\text { IL-1 } \\
\text { IL-3 }\end{array}$ & $\begin{array}{c}\text { M-CSF } \\
\text { GM-CSF } \\
\text { IL-3 }\end{array}$ \\
\hline $\begin{array}{l}\text { transcription } \\
\text { factors }\end{array}$ & $\begin{array}{c}\text { SCL } \\
\text { GATA-1,2 } \\
\text { c-Myc }\end{array}$ & $\begin{array}{c}\text { SCL } \\
\text { C/EBPa } \\
\text { c-Myb }\end{array}$ & $\begin{array}{l}\text { PU.1 } \\
\text { AML1 } \\
\text { C-Myb } \\
\text { EGR-1 }\end{array}$ \\
\hline
\end{tabular}


Two recent findings have enriched the study of monocyte differentiation. The stochastic quality of lineage commitment has been challenged by data showing that macrophage colony stimulating factor (M-CSF), the primary survival, differentiation, and proliferation factor for monocytes and macrophages, can instruct myeloid cell fate through promoter activation of the transcription factor PU.1 (Mossadegh-Keller et al., 2013). PU.1 is an essential regulator of myeloid lineage commitment (McKercher et al., 1996; DeKoter et al., 1998; Nerlov and Graf, 1998). Second, a population of highly proliferative cells was identified in the bone marrow and spleen that differentiated to monocytes and macrophages but not dendritic cells and granulocytes. Referred to as common monocyte progenitors ( $\mathrm{cMoP})$, this population appears to mark the branch point from dendritic cells and monocytes (Hettinger et al., 2013). Our current understanding places the cMoP population as the immediate precursor to monocytes (Figure 1.3).

\subsection{Differentiation of progenitor cells to committed monocytes}

\subsubsection{Regulated expression of surface antigens}

The progression of monocyte progenitor to monocyte in the BM marks a significant stage of differentiation. Hallmarks of this transition include reduced proliferative capacity, commitment to the monocyte lineage, and the regulated expression of surface antigens, the latter conveying functionality in addition to hierarchy (Hettinger et al., 2013) (Figure 1.3). A gain in the expression of CD11b (integrin $\alpha \mathrm{M} \beta 2$ ), under control of the transcription factor PU.1, is initiated at this stage and serves as a primary marker for all myeloid lineage cells (Pahl et al., 1993). CD11b regulates firm adhesion of blood monocytes to the endothelium in peripheral tissues (Shi and Simon, 2006; Herter and Zarbock, 2013). Expression and clustering of CD11b also leads to downregulation of the transcription factor foxp1, removal of foxp1-mediated transcriptional inhibition at the macrophage colony stimulating factor receptor locus, and subsequent enhanced 
Figure 1.3 Monocyte differentiation occurs independently in the BM and blood. Macrophage-dendritic cell progenitors (MDP) gain lineage commitment during differentiation to common monocyte progenitor cells $(\mathrm{cMoP})$. Ly6 $\mathrm{C}^{\text {high }}$ monocytes are derived from $\mathrm{cMoP}$, and either differentiate in the $\mathrm{BM}$ to $\mathrm{Ly} 6 \mathrm{C}^{\text {low }}$ monocytes or migrate across an endothelial cell barrier to the blood, whereupon they persist or differentiate to Ly $6 \mathrm{C}^{\text {low }}$ monocytes. Surface antigens that define the MDP, cMoP, Ly6C $\mathrm{C}^{\text {high }}$, and $\mathrm{Ly} 6 \mathrm{C}^{\text {low }}$ populations are listed. 


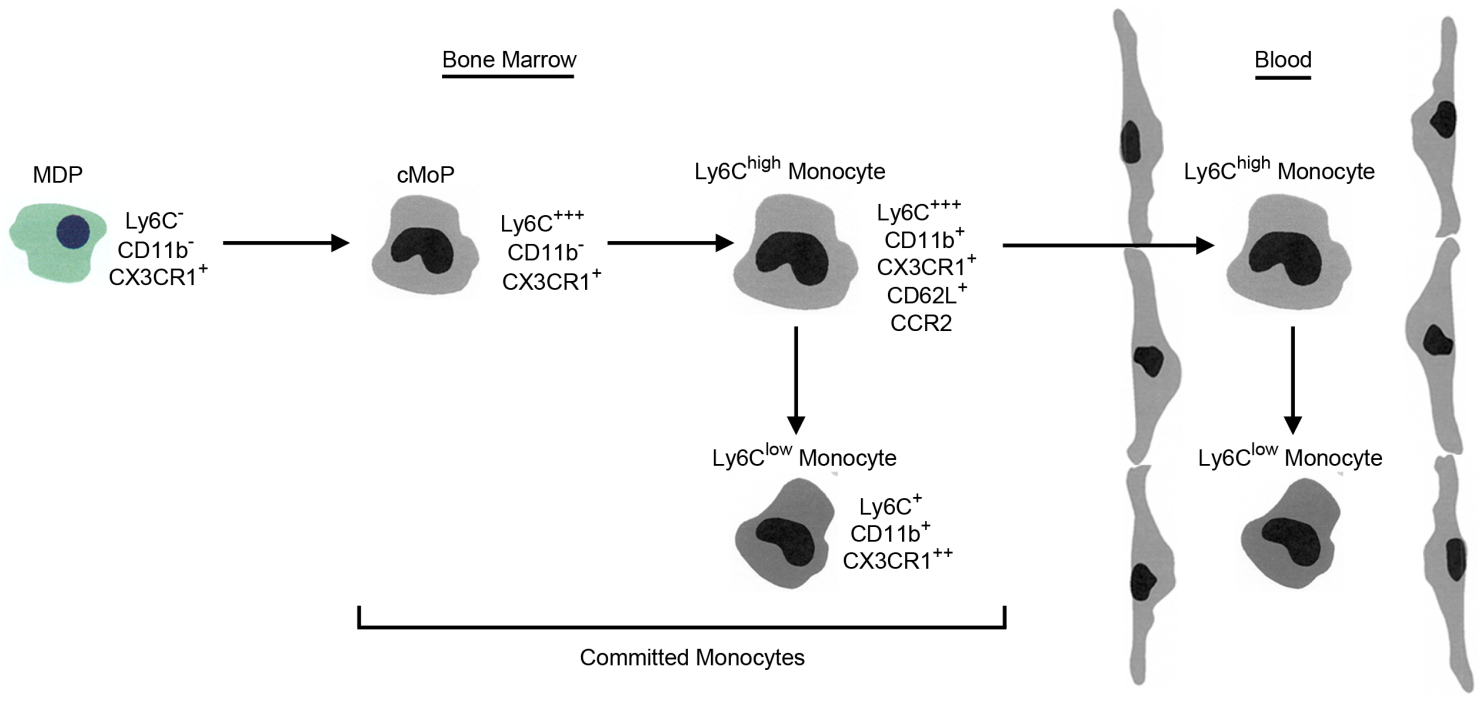


surface expression of M-CSF receptor (M-CSFR) (Shi et al., 2008). This indicates a role not only for $\mathrm{CD} 11 \mathrm{~b}$ in adhesion and trafficking but also for integrin-mediated regulation of monocyte differentiation. Ly6C is glycosyl phosphatidyl inositol (GPI)-anchored surface glycoprotein expressed in monocytes but downregulated during differentiation (Leenen et al., 1994; Jutila et al., 1988). It is also expressed on a subset of $\mathrm{CD}^{+} \mathrm{T}$ lymphocytes and endothelial cells. In purified $\mathrm{CD}^{+} \mathrm{T}$ cells, it was shown that Ly6C mediated-adhesiveness was reduced with the inhibition $\alpha 4 \beta 1$ integrin (Hanninen et al., 1997). CX3CR1, a prototypical 7-transmembrane Gprotein-couple receptor, is expressed in a variety of cell types, including natural killer (NK) cells, T cells, and monocytes (Imai et al., 1997). Binding of CX3CR1 to the ligand fractalkine on activated endothelial cells regulates monocyte adhesion and migration (Goda et al., 2000). The expression of the chemokine receptor CCR2 on a subset of monocytes permits motility toward monocyte chemoattractant protein 1 (MCP-1) (Cambien et al. 2001). This is evident under inflammatory conditions at peripheral sites (Imhof and Aurrand-Lions, 2004; Kamei and Carman, 2010). CD62L (L selectin) mediates monocyte rolling on activated endothelium and is functional on the same population of monocytes that expresses high levels of Ly6C and CCR2 (Auffray et al., 2009; Xu et al., 2008). Combined, these surface antigens provide a means to distinguish monocyte populations.

\subsubsection{Establishment of two distinct monocyte populations}

From this classification scheme has emerged a dichotomy of related monocyte populations. $\mathrm{CD} 11 \mathrm{~b}^{+}$Ly6 $\mathrm{C}^{\text {high }} \mathrm{CX} 3 \mathrm{CR} 1^{\text {low }}$ monocytes in the $\mathrm{BM}$ are committed cells that maintain low proliferative capacity (Hettinger et al., 2013). They persist as functional monocytic cells that, under appropriate stimuli, egress from the BM in a CCR2-dependent manner and repopulate the circulating monocyte pool (Serbina and Pamer, 2006). A seminal series of experiments conducted over a decade ago utilizing reporter mice that had green fluorescent protein (GFP) inserted into

the $\mathrm{CX} 3 \mathrm{CR} 1$ locus $\left(\mathrm{CX} 3 \mathrm{CR} 1^{\mathrm{GFP} /+}\right)$ revealed that circulating murine monocytes were in fact two 
discrete populations (Geissmann et al., 2003) (Figure 1.4). Several observations made at that time have persisted. The $\mathrm{Ly} 6 \mathrm{C}^{\text {high }}$ population was short-lived relative to Ly6 $\mathrm{C}^{\text {low }}$ monocytes. Ly6C ${ }^{\text {high }} \mathrm{CX} 3 \mathrm{CR} 1{ }^{\text {low }} \mathrm{CCR} 2{ }^{+} \mathrm{CD} 62 \mathrm{~L}^{+}$monocytes preferentially homed to inflamed sites, whereas Ly6 ${ }^{\text {low }} \mathrm{CX} 3 \mathrm{CR} 1{ }^{\text {high }} \mathrm{CCR} 2{ }^{-} \mathrm{CD} 62 \mathrm{~L}^{-}$monocytes were found in uninflamed tissues. During inflammation, the Ly6 $\mathrm{C}^{\text {high }}$ population differentiated into functional dendritic cells (DC). A small percentage of Ly6 $\mathrm{C}^{\text {low }}$ monocytes did express markers for DC, although it was not confirmed that these cells were functional. Lastly, CX3CR1 expression was involved in tissue engraftment of Ly6 $\mathrm{C}^{\text {low }}$ monocytes. These experiments addressed the major points that would drive future investigations, including the relationship of $\mathrm{Ly} 6 \mathrm{C}^{\text {high }}$ to $\mathrm{Ly} 6 \mathrm{C}^{\text {low }}$ monocyte populations, the factors that regulate the accumulation of monocytes in tissues, and the fate of tissue resident effector populations. Gene expression profiles of the two murine subsets depicted in Figure 1.4 differ significantly but display a high degree of homology with the two parallel monocyte subsets in humans, suggesting a high degree of evolutionary conservation within each subset (Ingersoll et al., 2010).

\section{4 $\mathrm{Ly6C}^{\text {high }}$ and $\mathrm{Ly6C}^{\text {low }}$ monocytes}

\subsubsection{Generation of $\mathrm{Ly}^{\mathrm{low}} \mathrm{C}^{\text {low }}$ monocytes}

Several studies in the subsequent decade using monocyte depletion techniques and reporter mice indicated that the Ly $6 \mathrm{C}^{\text {low }}$ monocyte pool is established through differentiation of Ly $6 \mathrm{C}^{\text {high }}$ monocytes in vivo (Sunderkotter et al., 2004; Varol et al., 2009). This is consistent with results from the initial experiments that identified and characterized ER-MP20 (Ly6C) (Leenen et al., 1990). Treatment of BM cells with M-CSF led to reduced Ly6C expression over time. Recently, three key conclusions have been made utilizing mouse models that have different fluorescent reporter constructs knocked into the CX3CR1 locus. Use of these mice permitted the tracking of 
Figure 1.4 Surface antigens distinguish $\mathrm{Ly}^{\mathrm{C}} \mathrm{C}^{\text {high }}$ and $\mathrm{Ly}^{\mathrm{low}}$ monocyte populations. Ly6 $\mathrm{C}^{\text {high }}$ monocytes are $\mathrm{CD} 11 \mathrm{~b}^{+} \mathrm{Ly} 6 \mathrm{C}^{+} \mathrm{CCR} 2^{+}$and express the adhesion molecules CD31, LFA-1, VLA-4 and CD62L. Ly6C ${ }^{\text {low }}$ monocytes are comparatively smaller and lack expression of Ly6C, CCR2, and CD62L. They are characterized by a high expression of CX3CR1. 
Ly6C ${ }^{\text {high }}$ Monocyte (inflammatory)

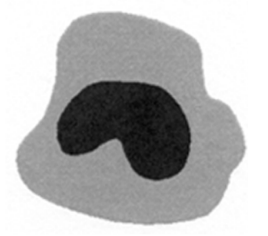

Ly6C ${ }^{\text {low }}$ Monocyte (resident)

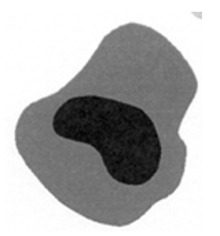

high

low

$+$

Adhesion Molecules

CD31

LFA1

VLA4

L-selectin (CD62L)
$++$

$+$

$+$

$+$
$+$

$++$

$+$ 
steady state monocyte differentiation as a function of CX3CR1 and Ly6C (Yona et al., 2013). First, Ly6C ${ }^{+}$blood monocytes were shown to be the immediate precursors of Ly6 $\mathrm{C}^{-}$monocytes. The generation of a Ly6C blood monocyte pool required the emigration of $\mathrm{Ly}^{-} \mathrm{C}^{+}$monocytes from the BM followed by the differentiation of this population (Figure 1.3). Second, the Ly6C $\mathrm{C}^{+}$ and $\mathrm{Ly}_{6} \mathrm{C}^{-}$monocyte populations in the blood and $\mathrm{BM}$ were shown to be established and maintained independently. Third, $\mathrm{Ly} 6 \mathrm{C}^{+}$monocytes were shown to have a shorter half-life $(\sim 20$ hours) in circulation compared to $\mathrm{Ly}^{-} \mathrm{C}^{-}$monocytes (2.2 days). Also of note was that, upon Ly $6 \mathrm{C}^{+}$monocyte depletion, the half-life of Ly6C monocytes increased to 11 days. Combined with results from an earlier study showing that the $\mathrm{Ly} 6 \mathrm{C}^{-}$population is reduced through neutralization of the M-CSF receptor, it was hypothesized that $\mathrm{Ly}_{6 \mathrm{C}} \mathrm{C}^{+}$monocytes function as an M-CSF sink and limit availability of this survival factor to $\mathrm{Ly}^{6} \mathrm{C}^{-}$monocytes (MacDonald et al., 2010). This would allow persistence of the $\mathrm{Ly} 6 \mathrm{C}^{-}$pool under circumstances when the $\mathrm{Ly} \mathrm{C}^{+}$ precursor monocyte population is reduced. If correct, this would suggest that the MPS evolved to maintain $\mathrm{Ly} 6 \mathrm{C}^{+}$and $\mathrm{Ly} 6 \mathrm{C}^{-}$monocytes concurrently and hence suggests that the populations are functionally distinct.

\subsubsection{Ly6C $^{\text {high }}$ monocyte trafficking to peripheral sites}

Ly6 $\mathrm{C}^{+}$blood monocyte trafficking to peripheral tissues is integral to the inflammatory response and represents the classical model for transmigration of circulating monocytes into peripheral tissue (Shi and Pamer, 2011; Kamei and Carman, 2010) (Figure 1.5A). Initiation of monocyte rolling along the surface of activated endothelium occurs through selectin-mediated interactions, including those involving CD62L (Beekhuizen and Van Furth, 1993; Imhof and Aurrand-Lions, 2004; Spertini et al., 1996; Ley, 2003). The subsequent activation of chemokine receptors on monocytes in response to the local milieu of factors leads to an increase in the affinity, through conformational changes, and avidity, through clustering, of integrins (Laudanna et al., 2002). CCL2 (MCP-1) is the prototypical chemokine involved in inflammation and disease, and binding 
to CCR2 activates the MAP kinase and PI3-kinase pathways (Cambien et al., 2001; Imhof and Aurrand-Lions, 2004). The internal cellular factors that regulate the chemokine-induced activation of integrins remain elusive (Herter and Zarbock, 2013; Laudanna et al., 2002). Rho mediates binding of monocytes to the endothelium and is a strong candidate as a regulator of chemokine receptor-integrin signaling (Wojciak-Stothard et al., 1999; Hotchin and Hall, 1995). Specificity of integrin binding to extracellular matrix (ECM) components or cell-associated receptors is assigned through the arrangement of one $\alpha$ and one $\beta$ chain (Miranti and Brugge, 2002). The avidity of $\beta 1$ and $\beta 2$ integrins can be regulated in response to chemokine stimulation (Weber et al., 1996). Enhanced integrin engagement permits the crawling phenotype required during the initial stages of diapedesis, or monocyte migration across the vascular endothelium. Evidence suggests that very late antigen 4 (VLA-4; $\alpha 4 \beta 1)$ and lymphocyte function associated antigen 1 (LFA-1; $\alpha \mathrm{L} \beta 2$ ) regulate the early and late stages of monocyte arrest and migration, respectively (Weber and Springer, 1998; Herter and Zarbock, 2013; Schenkel et al., 2004). LFA$2(\alpha \mathrm{M} \beta 2)$ is involved in Ly6C $\mathrm{C}^{+}$migration as well. Shear stress has also been implicated in the induction of monocyte adhesion through the modulation of integrin affinity (Gerszten et al., 1999; Alon and Dustin, 2007).

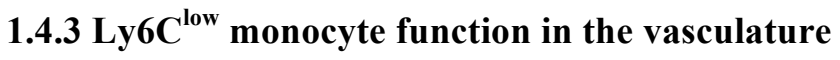

Ly6C $\mathrm{C}^{-}$blood monocytes are distinguished not only by surface antigen expression but by function. Roughly five years after blood monocyte dichotomy was firmly established, Auffray and colleagues first characterized Ly6 $\mathrm{C}^{-}$monocyte behavioral phenotype utilizing CX3CR $1{ }^{\mathrm{GFP} /+}$ mice (Auffray et al., 2007). They documented that, in steady state, this subset appeared to crawl along vessels in the dermis and mesentary as though the monocytes were patrolling the endothelium (Figure 1.5B). Migration along the endothelial barrier could occur counter to blood flow, in various patterns, and rarely resulted in extravasation. The average rate of movement was $12 \mu \mathrm{m} / \mathrm{min}$, which is relatively slow considering the average size of monocytes is $\sim 20 \mu \mathrm{m}$. 
Figure 1.5 $\mathrm{Ly}^{\mathrm{C}} \mathrm{C}^{\text {high }}$ and $\mathrm{Ly}^{\mathrm{low}}$ monocytes utilize different mechanisms for attachment and trafficking on the endothelium. (A) Ly6 $\mathrm{C}^{\text {high }}$ monocyte rolling and adhesion is mediated by CD62L (L-selectin) and CCR2. CCR2, LFA-1, and VLA-1 regulate firm adhesion and migration on the endothelium. CD31 (PECAM) plays a critical role in migration across the endothelial cell barrier. (B) Ly6 $\mathrm{C}^{\text {low }}$ monocytes utilize CX3CR1 and LFA-1 for adhesion. The patrolling behavior of Ly6 $\mathrm{C}^{\text {low }}$ monocytes is regulated by CX3CR1 and LFA-1. 
A.

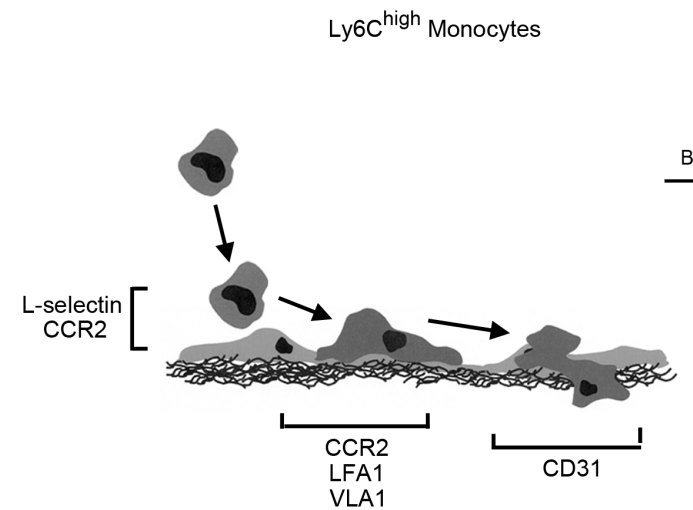

B.

$$
\text { Ly6c }{ }^{\text {low }} \text { Monocytes }
$$

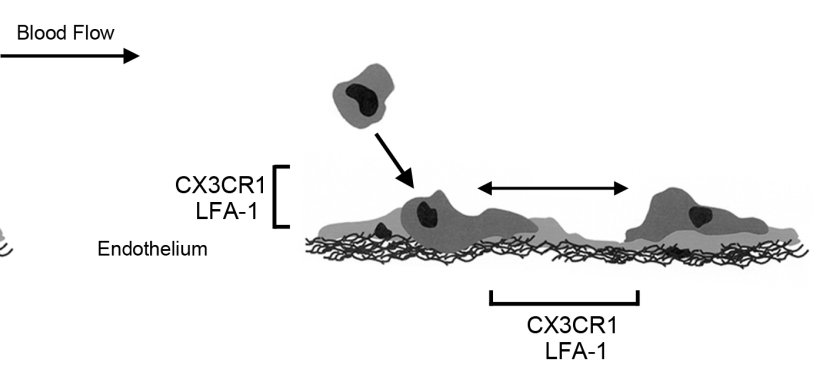


Intravital microscopy beautifully illustrated that after one hour, a significant proportion of the vessel surface had been covered due to the combined efforts of all adherent Ly6 $\mathrm{C}^{-}$monocytes. Signaling through LFA-1 and CX3CR1 were critical for the crawling behavior. The role of VLA1 and VLA-2 in this process was not determined. Following the induction of inflammation, Ly $6 \mathrm{C}^{-}$ monocytes were shown to rapidly extravasate into the tissue, prior to the arrival of neutrophils and Ly $6 \mathrm{C}^{+}$inflammatory monocytes. They produced TNF- $\alpha$, a factor commonly associated with an inflammatory response. Over time, however, the genetic profile of this population switched to one consistent with tissue repair. A subsequent study revealed that in the kidney vasculature, Ly6C $\mathrm{C}^{-}$patrolling monocytes are in flux with the circulating population and have an average dwell time of 9 minutes on the endothelium (Carlin et al., 2013a). It was estimated that there were proportionally three times as many $\mathrm{Ly}^{-} \mathrm{C}^{-}$monocytes in circulation relative to the adherent population. During crawling, the monocytes extended filipodia-like structures and could scavenge microparticles that had been experimentally attached to the endothelium. This population also recruited neutrophils in a toll-like receptor 7 (TLR-7) dependent manner.

\subsection{Monocyte fate in tissues}

The fate of circulating monocytes subsequent to extravasation has been widely studied. Several examples highlight the variability and complexities of monocyte trafficking to peripheral tissues. Epithelial turnover and the presence of commensal gut microbiota are hallmarks of steady state colonic biology. Ly $6 \mathrm{C}^{+}$monocytes are continually recruited to a healthy gut, persist for short term as $\mathrm{CX} 3 \mathrm{CR} 1^{+}$macrophages, and secrete the anti-inflammatory cytokine IL-10 (Zigmond and Jung, 2013). Evidence suggests that recruitment is largely dependent on CCR2 expression, which is likely a result of basal levels of CCL2 due to low grade inflammation, and that formation of transepithelial dendrites (TED), which are presumed to allow sampling of the gut fauna, requires 
CX3CR1. This population is in contradiction with the established paradigm, which suggests that $\mathrm{CCR} 2$ and $\mathrm{CX} 3 \mathrm{CR} 1$ are expressed and functional on $\mathrm{Ly}_{6 \mathrm{C}}^{+}$and $\mathrm{Ly}_{6} \mathrm{C}^{-}$monocyte subsets, respectively. During colitis, $\mathrm{Ly} 6 \mathrm{C}^{+}$monocyte recruitment was seen to increase, the cells expressed toll-like receptors (TLR) and pro-inflammatory cytokines, and over time differentiated into antigen presenting cells (Zigmond et al., 2012). They became functional effector cells appropriately suited to act in the inflammatory microenvironment. These results suggest that, in the colon, monocyte/macrophage phenotype is largely dependent on the local stimuli. Recent data indicate that $\mathrm{Ly} 6 \mathrm{C}^{+}$monocyte populations exist extravascularly in tissues but do not undergo differentiation to become macrophages or antigen presenting cells (Jakubzick et al., 2013). Monocytes were shown to exist in the lung and skin and were capable of migrating from these tissues to lymph nodes via the lymphatics. During this process, the cells never acquired a phenotype consistent with that of macrophages, suggesting that the infiltrating monocytes either did not respond to environmental cues that drive differentiation, or they utilized signaling pathways that dampen differentiation.

The heart has served as a second model to study monocyte trafficking given that it harbors MPS populations that maintain steady state, assist in the resolution of tissue following damage, and contribute to pathology (Nahrendorf and Swirski, 2013; Ley et al., 2011). A study conducted in the healing myocardium following infarct revealed that $\mathrm{Ly} \mathrm{C}^{+}$recruitment preceded the arrival of Ly6C $\mathrm{C}^{-}$monocytes (Nahrendorf et al., 2007). The temporal regulation of this process was mediated through a shift in chemokine expression in the myocardial tissue. During the early phase, elevated levels of the pro-inflammatory chemokines CCL2 and macrophage inflammatory protein $-1 \alpha$ (MIP-1 $\alpha$ ) resulted in the recruitment of Ly6C $\mathrm{C}^{+}$monocytes. These factors were subsequently downregulated while fractalkine expression increased, leading to the second phase marked by Ly6C $\mathrm{C}^{-}$monocyte recruitment. This experiment illustrated the importance of chemokine-mediated signaling to monocyte recruitment. 
Other studies have highlighted the differentiation potential of recruited monocytes. In the lung, it was shown that $\mathrm{Ly} 6 \mathrm{C}^{\text {high }}$ and $\mathrm{Ly} 6 \mathrm{C}^{\text {low }}$ monocytes can differentiate into pulmonary dendritic cells during steady state and inflammation (Landsman et al., 2007). However, only the Ly6C $\mathrm{C}^{\text {low }}$ monocytes, either through recruitment or following the loss of Ly6C, could differentiate into lung macrophages. In the fibrotic kidney, recruited $\mathrm{Ly} 6 \mathrm{C}^{+}$monocytes from the bone marrow lost Ly6C surface expression and persisted as three populations distinguishable by either a pro or antiinflammatory transcriptional profile (Lin et al., 2009). The theme of MPS biology, one comprised of complexity, responsiveness, plasticity, and movement, is illustrated in the preceding examples.

\subsection{Macrophages}

\subsubsection{Derivation of tissue resident macrophages}

The terminal stages of MPS differentiation culminate in the generation of macrophages that reside in peripheral tissues. Studies from the 1960s and the next two decades investigating early stage monocyte differentiation, the kinetics of tissue macrophage repopulation by circulating monocytes, and macrophage proliferation in tissues illuminated the concept of multiple origins for tissue macrophage populations (Takahashi 2000). Data from three independent studies conducted within the last three years have provided evidence that a large proportion of tissue resident populations, including those in the pancreas, liver, brain, and skin are derived from embryonic yolk sac progenitors (Schulz et al., 2012; Ginhoux et al., 2010). In addition, Langerhans cells in the dermis were shown to have fetal liver and embryonic origins (Hoeffel et al., 2012). There is evidence that the generation of tissue macrophage populations is due predominantly to the proliferative effects of colony stimulating factors and not from the recruitment of circulating monocytes (Hashimoto et al., 2013). These studies provide the framework for a system in which steady state tissue homeostasis is maintained by resident 
macrophages established during development, and macrophages derived from circulating monocytes participate predominantly in response to tissue insult and disease resolution.

\subsubsection{Regulation of MPS populations by colony stimulating factors}

The dependence on colony stimulating factors (CSF) for differentiation, survival, and proliferation is one constant across all mononuclear phagocytes (Barreda et al., 2004). This is true in the bone marrow, blood, and peripheral tissues, where regulation of MPS biology is achieved through cell surface receptor binding and the subsequent activation of intracellular signaling cascades and transcriptional activation or repression. This process has been relatively well delineated in the bone marrow during monocyte differentiation, and evidence indicates that control exists both at the level of receptor expression and the availability of soluble growth factors in the local milieu. For cells of the MPS, the most important regulatory factors are macrophage colony stimulating factor (M-CSF), also called colony stimulating factor 1 (CSF1), and granulocyte macrophage colony stimulating factor (GM-CSF). M-CSF is ubiquitously expressed in steady state, and activation of its cognate receptor leads to receptor autophosphorylation and downstream signaling mediated by Src family kinases and phosphatidylinositol 3 kinase (PI3K) (Hamilton, 2008). The severe abnormalities observed in mice that have a homozygous null mutation for $C s f 1\left(C s f 1^{o p / o p}\right)$ highlight a prominent role in tissue homeostasis (Wiktor-Jedrzejczak et al., 1990). These mice are osteopetrotic due to the absence of bone reabsorption by osteoclasts (bone macrophages) and display compromised hematopoiesis. CsflRnull mice present with the loss of microglia (brain macrophages) and Langerhans cells (liver macrophages) in addition to the defects observed in op/op mice (Dai et al., 2002). This is attributed to the role of interleukin 34 (IL-34), a cytokine that binds to CSF1R as well (Lin et al., 2008). Expression of GM-CSF appears to be elevated during inflammation, although it also appears to play a prominent role in regulating hematopoiesis at steady state (Lenzo et al., 2012). The systemic loss of GM-CSF results most notably in a compromised alveolar macrophage pool. 
Ligand binding to its cognate non-kinase transmembrane receptor induces janus kinase 2 (JAK2) and signal transducer and activator of transcription 5 (STAT5) activation, as well as pathways mediated by mitogen-activated protein kinase (MAPK) and PI3K (Hamilton 2008).

It is well established that both M-CSF and GM-CSF induce the differentiation of BM monocyte progenitor cells to macropahges in vitro (Hamilton and Achuthan, 2013). M-CSF is a more potent factor relative to GM-CSF, as the latter also induces differentiation of granulocytic myeloid cells. Additionally, a profile of cytokines produced from BM cultured in the presence of GM-CSF suggests that this factor promotes macrophages to adopt an inflammatory phenotype, whereas MCSF treatment potentiates a comparably more restorative, or growth-oriented, genetic signature (Fleetwood et al., 2007). These two characteristics are revealing when placed in context with results from a study that utilized a CSF1R blocking antibody to assess the role CSF1 on mononuclear phagocyte population dynamics. To track this lineage, the authors utilized MacGreen mice, which express enhanced green fluorescent protein (EGFP) under the control of the $\operatorname{Csf1R}$ promoter (Sasmono et al., 2003). Expression of CsflR occurs early in monocyte differentiation and thus fluorescence in MacGreen mice is a surrogate marker for all mononuclear phagocytes. A caveat to this model is that $C S f 1 R$ mRNA can be transcribed in neutrophils as well (Sasmono et al., 2007). It was shown that $\alpha$-CSF1R antibody treatment did not significantly impact monocytopoiesis or the overall percentage of $\mathrm{GFP}^{+} \mathrm{Ly}_{6 \mathrm{G}^{+}}$and $\mathrm{GFP}^{+} \mathrm{CD} 11 \mathrm{~b}^{+}$populations in the BM, blood, and spleen (MacDonald et al., 2010). However, there was a lower proportion of $\mathrm{GFP}^{+} \mathrm{F} 4 / 80^{\text {high }}$ cells in the blood of mice that received the blocking antibody. Monocyte differentiation to macrophage correlates with increased expression of F4/80. The blood also had fewer $\mathrm{GFP}^{+} \mathrm{GR}-1^{-}$cells in circulation, indicating fewer more differentiated monocytes. The authors concluded that treatment with antibody against CSF1R blocked the maturation of GR $1^{+}$ monocytes into GR-1 $1^{-} 4 / 80^{\text {high }}$ monocytes. In tissues, a similar reduction in the more mature GR1 ${ }^{-}$resident macrophage population was found concomitant with an increase in the percentage 
of $\mathrm{GR}^{+}$inflammatory monocytes. Antibody treatment did not influence recruitment of monocytes during inflammation. Two main conclusions were derived from this study. First, signaling through CSF1R is only necessary for monocyte maturation and thus impacts the generation of $\mathrm{GR}^{-}$blood and tissues monocytes/macrophages. Second, the production of monocytes and the function of less differentiated monocytes is not regulated by CSF 1R signaling. A model incorporating these results places GM-CSF as a factor expressed during inflammatory events that regulate the function of less differentiated, inflammatory monocytes. It induces the early stages of monocyte differentiation in the BM. Conversely, CSF1 is ubiquitously expressed. It is critical for the differentiation of $\mathrm{Ly}_{6 \mathrm{C}} \mathrm{C}^{+}$monocytes to $\mathrm{Ly} 6 \mathrm{C}^{-}$monocytes and tissue resident macrophage function. Expression of CSF1 during steady state is essential, given that macrophages reside in all tissues. If placed on the scale of monocyte differentiation, GM-CSF and M-CSF would predominate in the early and late stages, respectively.

\subsubsection{Coordinated effect of multiple stimuli on the MPS}

Changes in cell phenotype following activation of cell surface receptors are a hallmark of the MPS. The integration of multiple stimuli can dramatically alter MPS biology relative to the outcome achieved in the presence of individual factors. One example of this involves interleukin 6 (IL-6). Signaling through IL-6 is initiated upon ligation with the receptor complex glycoprotein 130 (gp130) and IL-6 receptor $\alpha(\mathrm{IL}-6 \mathrm{R} \alpha)$ (Eulenfeld et al., 2012). This results in activation of the JAKs and downstream signaling through STAT, MAPK, and PI3K. Lineage-specific expression of IL-6R $\alpha$ is a key regulatory checkpoint given the ubiquitous expression of gp 130. It has been documented that IL-6 functions in both a pro and anti-inflammatory capacity and can stimulate monocyte migration (Clahsen and Schaper, 2008). Il-6 has also been shown to

synergize with IL-3 and promote the proliferation of hematopoietic progenitor cells (Ikebuchi et al., 1987). Bone marrow cells cultured with IL-6 alone results in high levels of cell death (Marigo et al., 2010). When added in combination with GM-CSF, IL-6 induces myeloid lineage cell 
differentiation that results in monocytic and granulocytic populations. Culture of BM cells with GM-CSF maintains cell viability but results in proportionally more granulocytic cells than when combined with IL-6. These results suggest that GM-CSF is sufficient to stimulate differentiation but its ability to regulate lineage specificity is altered in the presence of IL-6. The coordination of IL-6 and M-CSF signaling has been documented as well. In co-culture, monocytes induced IL-6 secretion by fibroblasts (Chomarat et al., 2000). Exposure to fibroblast-derived IL-6 increased expression of CSF1R on monocytes, resulting in enhanced CSF1R signaling through autocrine CSF1. This interaction pushed monocyte differentiation to macrophages rather than dendritic cells. In Chapter 2, I will address $\mathrm{Ly} 6 \mathrm{C}^{+} \mathrm{F} 4 / 80^{-}$monocyte differentiation in response to all three factors.

\subsection{Perspective on mononuclear phagocytes and tumors}

Animal models and the controlled manipulation of mononuclear phagocytes in vitro have allowed assessment of monocyte differentiation, trafficking, and effector function during both steady state and disease (Frese and Tuveson, 2007). The examples above illustrate that differentiation state and phenotype of mononuclear phagocytes are driven by exposure to microenvironmental stimuli, and conversely the microenvironment is shaped by the behavior of these cells (Tlsty and Coussens, 2005). During the past few decades, model systems have been developed to study the interaction of mononuclear phagocytes with other components of the tumor microenvironment. This is significant for three reasons. First, we appreciate that, in most instances, solid tumors are complex entities with a significant stromal (non-transformed) compartment. Second, mononuclear phagocytes localize to tumors and persist. Third, as highly plastic, non-transformed cells, they hold the potential to be modulated by therapeutic means (Shiao et al., 2011). 
The documented interaction of leukocytes and tumors is historic. In the mid1800s, the pathologist Rudolph Virchow first noted the presence of non-cancerous phagocytic cells alongside transformed cells in tumor specimens and linked cancer to the inflammatory response (Balkwill and Mantovani, 2001). This seminal observation initiated 150 years of research to determine if blood cells modulate tumor progression. The results overwhelmingly indicate that the innate arm of human immunity dictates cancer progression. Experiments in animal models suggest that the requisite machinery to exert this influence exists throughout the animal kingdom. Drosophila melanogaster hemocytes, which are circulating immune cells and are thought to share a common evolutionary origin with mammalian innate blood cells, were shown to increase in number following the experimental induction of tumor growth, detect basement membrane disruption at the tumor site, and elicit an immune response. JAK/STAT signaling was activated during this process, which is a commonly regulated pathway in cytokine-mediated immunity in humans (Pastor-Pareja et al., 2008). Evidence of neoplastic growth is documented across all metazoans, excluding Porifera (sponges), although it has yet to be verified that a cell-mediated response exists under these conditions (Robert, 2010). This does suggest, however, that all animals have had to address and resolve aberrant growth throughout evolution. Given that invertebrates harbor phagocytic cells and that they contribute to the immune response in the context of more thoroughly studied diseases, it is reasonable to hypothesize that the relationship of tumor and phagocyte is ancient.

The MPS in mice and humans has been implicated in all stages of tumor development (Laoui et al., 2011; Chioda et al., 2011). The regulated progression of tumors results from the accumulative effect of MPS recruitment and effector function. Monocytes in circulation respond to tumorderived factors, adhere to the endothelium, transmigrate across the endothelium, and migrate within the tumor bed to defined sites. This is a directed response dependent on the abilities of tumors to secrete factors and circulating monocytes to effectively respond to these stimuli 
(Balkwill, 2004). Circulating monocytes are not terminally differentiated. MPS cell populations at various stages of differentiation have unique effector capabilities that are concomitant with the acquired stage of differentiation (Gabrilovich et al., 2012). Phenotypic plasticity is a hallmark of terminally differentiated macrophages (Mantovani et al., 2007; Porcheray et al., 2005; Stout et al., 2005; Stout and Suttles, 2004). Gene expression profiles of macrophages in the tumor microenvironment can be similar to those of macrophages involved in development and growth (Ojalvo et al., 2009). The outcome of this is that macrophages can both combat and potentiate tumor progression.

\subsection{Tumor associated macrophages (TAM) and myeloid derived suppressor cells (MDSC)}

\subsubsection{Functional attributes of TAM and MDSC}

Evidence exists that accumulation of MPS populations in the tumor microenvironment correlate with poor patient prognosis or tumor stage. A comprehensive summary of tumor associated macrophage (TAM) accumulation was completed by Bingle in 2002 across a range of tissues (Bingle et al., 2002). Diaz-Montero published a complementary summary of less differentiated myeloid cells in 2009 (Diaz-Montero et al., 2009). These studies in total suggest that differentiation status does not in itself correlate with severity of disease, and indicate rather that the behavior or function of myeloid cell populations is the dominant influencing factor.

Two monocytic cell populations have been targeted for study due to their propensity to accumulate in tumor-bearing animal models and cancer patients. Monocytic myeloid derived suppressor cells (M-MDSC) and tumor associated macrophages (TAM) occupy different regions on the differentiation spectrum. M-MDSC are described as less differentiated, Ly6 $\mathrm{C}^{\text {high }}{ }^{2 y} 6 \mathrm{G}^{-}$ myelomonocytic cells, whereas TAM are characterized as differentiated monocytic cells that have low Ly6C and high F4/80 expression. Numerous examples in mice highlight TAM function 
(Allavena et al., 2008; Pollard, 2004; Qian and Pollard, 2010). TAM can have a direct action on tumors through their ability to mediate phagocytosis of tumor cells. However, TAM are thought in most cases to enhance tumor progression through the secretion of growth factors that have a mitogenic and migratory influence. Work from the Condeelis and Pollard groups has shown that a paracrine loop can develop between breast carcinoma cells and TAM involving the secretion of M-CSF and epidermal growth factor (EGF), respectively (Goswami et al., 2005). This mutual exchange enhances the directed motility and invasion of the tumor cells. Modulation of the extracellular matrix due the secretion of matrix metalloproteases by TAM can stimulate the release and availability of sequestered growth factors. The ability of tumors to grow in volume is dependent upon adequate oxygen perfusion, and there is evidence that TAM secrete high levels of vascular endothelial growth factor (VEGF), a potent angiogenic factor (Pollard 2004). This activity, or angiogenic switch, induces vessel development, permits tumors to grow, and enhances metastasis. M-MDSC are impactful through shared and divergent mechanisms (Sica and Bronte, 2007). In a murine model of breast carcinoma, it was shown that the proportion of MDSC in both peripheral blood and spleen increased with tumor burden (Melani et al., 2003). When isolated from the tumor tissue of tumor bearing mice and re-implanted with carcinoma cells, the MDSC promoted tumor growth and angiogenesis. M-MDSC have gained prominence in the tumor literature due to their potent inhibitory effects on cytotoxic $\mathrm{T}$ cells. Increased arginase expression is one mechanism utilized by M-MDSC, in addition to the production of immune-suppressive cytokines (Youn and Gabrilovich, 2010).

MDSC gained prominence over a decade ago with the observation that a significant number of human cancer patients had reduced levels of professional antigen presenting cells (dendritic cells; DC) that correlated with increased proportions of myeloid cells that lacked surface antigens commonly found on more differentiated hematopoietic cells (Almand et al., 2001). Referred to as lineage negative ( $\left.\mathrm{Lin}^{-}\right)$immature cells $(\mathrm{ImC})$, this population was further distinguished from DC 
by reduced expression of HLA-DR (MHC II). When peripheral blood was sorted and cultured in factors that supported growth of myeloid and erythroid cell colonies, Lin ${ }^{-H L A-D R}{ }^{-}$cells formed ten times as many colonies as the Lin ${ }^{-} \mathrm{HLA}_{-} \mathrm{DR}^{+}$DC population, suggesting that the Lin ${ }^{-H L A}$ $\mathrm{DR}^{-} \mathrm{ImC}$ population harbored more progenitor cells. The vast majority of blood $\operatorname{ImC}$ had differentiated beyond the earliest $\mathrm{CD} 4^{+}$progenitor cell, however, and roughly one third expressed M-CSF receptor, indicating that a significant proportion was of the mononuclear phagocyte lineage but less differentiated. The ex vivo culture of ImC in M-CSF resulted in approximately one third of the cells displaying the human monocyte/macrophage marker CD14 ${ }^{+}$, confirming that a proportion of the ImC was mononuclear phagocytes. Functionally, the HLA$\mathrm{DR}^{-}$population displayed a significantly reduced ability to stimulate allogenic $\mathrm{T}$ cells compared to the HLA-DR ${ }^{+}$DC population. What appeared to be a parallel population of suppressive cells in mice was identified around the same time (Bronte et al., 2000). It had been observed in tumor bearing or vaccine immunized mice that a decreased $\mathrm{CD}^{+} \mathrm{T}$ cell function or proportion correlated with the accumulation of $\mathrm{CD} 11 \mathrm{~b}^{+} \mathrm{Gr}-1^{+}$myeloid cells in secondary lymphoid organs. It was shown that systemic GM-CSF release was responsible for the increased proportion of $\mathrm{CD} 11 \mathrm{~b}^{+} \mathrm{GR}-1^{+}$cells. Termed iMacs, or inhibitory macrophages, this population included both mature myelo-monocytic (polymorphonuclear cells and monocytes) and immature myeloid cells. Ly6C, ER-MP58, and CD31 expression on a population subset indicated a less differentiated, or progenitor cell, phenotype. Additional evidence highlighted the inherent plasticity of the $\mathrm{CD} 11 \mathrm{~b}^{+} \mathrm{Gr}-1^{+}$population and confirmed they were not terminally differentiated cells. It was known at the time that cytokines produced by the T helper 1 (Th1) and T helper 2 (Th2) subsets of $\mathrm{CD} 4^{+} \mathrm{T}$ lymphocytes elicited different responses from macrophages. Th1 derived factors such as IL-12, interferon- $\gamma(\mathrm{IFN}-\gamma)$, and tumor necrosis factor- $\alpha$ (TNF- $\alpha)$ induced macrophages to become potent anti-microbial effectors through the upregulation of molecules for antigen presentation and $\mathrm{T}$ cell costimulation. Conversely, a phenotype consistent with wound healing or the resolution of inflammation was a consequence of mediators such as IL-4 secreted by Th2 
cells. What was revealed is that exposure of the $\mathrm{CD} 11 \mathrm{~b}^{+} \mathrm{Gr}-1^{+}$iMacs to Th1 or Th2 cytokines produced mononuclear phagocytes with divergent effector functions. Cells cultured in the absence of exogenous cytokine or with IL-4 were suppressive, whereas those cultured in Th1 cytokines displayed increased MHCII expression and cytolytic activity of allogeneic targets in mixed lymphocyte assays. Two significant conclusions could be drawn from this set of experiments. First, iMacs were not terminally differentiated. Second, although they were lineage committed, they retained functional plasticity. Additionally, the data suggested that the default pathway of these cells was more in line with immunosuppression. An enhanced cytolytic response was achieved only upon treatment with Th1 cytokines.

\subsubsection{Characterization of tumor associated monocyte populations}

Subsequent research has focused on the factors the drive MDSC accumulation, the mechanisms of MDSC suppressor activity, the delineation of MDSC populations, and the differentiation of MDSC in the context of other myeloid lineage cells. The significance of MDSC in cancer appears to be due to their persistence. These cells participate in the resolution of infection but do not exist at significant levels beyond the time course of the response. Evidence indicates that in tumor bearing animals, a systemic milieu of factors secreted by the tumor microenvironment inhibit MDSC differentiation and potentiate proliferation and survival, thus propagating a continued state of immune suppression (Kusmartsev and Gabrilovich, 2002; Gabrilovich and Nagaraj, 2009). The two main MDSC populations identified to date, granulocytic MDSC (G-MDSC) and monocytic MDSC (M-MDSC), have suppressive capabilities, although they utilize different mechanisms (Dolcetti et al., 2010; Youn et al., 2008). They are commonly distinguished by the relative level of Ly6C and Ly6G expression. Isolated cell populations that are $\mathrm{CD} 11 \mathrm{~b}^{+} \mathrm{Ly} 6 \mathrm{G}^{\text {high }} \mathrm{Ly} 6 \mathrm{C}^{\text {low }}$ are more granulocytic, suppress primarily through reactive oxygen mediated (ROS) mechanisms, and depend on close interaction with $\mathrm{T}$ cells for suppressive activity. CD $11 \mathrm{~b}^{+}$Ly6G $6 y 6 \mathrm{C}^{\text {high }} \mathrm{M}-$ MDSC tend to have greater immunosuppressive strength, function independent of contact with $\mathrm{T}$ 
cells, and frequently localize to the tumor microenvironment. Evidence from murine tumor models suggests that that the proportion of each population is variable. Maturational hierarchy has not been established in these two populations, and currently there is no defined differentiation pathway placing either G-MDSC or M-MDSC in context with other mononuclear phagocytes. As a group they appear to fall in between monocyte progenitors and differentiated tissue macrophages. Their role of shaping tumor biology, either through potentiating angiogenesis or blocking cytotoxic $\mathrm{T}$ cell activity, is broadly viewed as favorable from the perspective of the tumor. It can be problematic when surface antigens used to identify distinct populations of myeloid cells are present on multiple cell populations. For example, high Ly6C and low Ly6G expression on $\mathrm{CD}_{1} 1 \mathrm{~b}^{+}$cells signify a population of lineage committed mononuclear phagocytes that are in the early stages of differentiation. This population could function either as inflammatory monocytes or as M-MDSC, and the nomenclature indicates an anti-tumor or protumor functionality, respectively. Resolution of this complexity is more problematic given that the literature suggests inflammatory monocytes can play both roles (Augier et al., 2010; Pommier et al., 2013; Sanford et al., 2013). These discrepancies may stem from a lack of consistency in defining myeloid cell populations by surface antigen expression and are illustrative of the fact that monocyte differentiation is a continuum. Numerous studies show that M-MDSC can differentiate in the tumor microenvironment and become tumor associated macrophages (Kusmartsev and Gabrilovich, 2005; Van Ginderachter et al., 2006; Corzo et al., 2010; Ma et al., 2011; Movahedi et al., 2008). In a murine model of breast cancer, four mononuclear phagocyte populations distinguished by MHCII and Ly6C expression were derived from the in situ differentiation of Ly6C ${ }^{\text {high }}$ monocytes (Movahedi et al., 2010). This is not surprising considering that solid tumors have been modeled as developing organs, and similar to aforementioned examples in $\mathrm{Ly}_{6 \mathrm{C}} \mathrm{C}^{+}$and $\mathrm{Ly} 6 \mathrm{C}^{-}$monocyte recruitment during steady state and infection, the delineation of monocyte populations requires an understanding of both origin and function (Egeblad et al., 2010). Gene expression profiles indicate that mononuclear phagocyte populations 
do share genetic signatures, and it is suggestive that specific effector function can be assigned to different populations. Tie2-expressing monocytes (TEM) are a subset of pro-angiogenic mononuclear phagocytes that exist in tumors and in circulation (De Palma et al., 2007). Isolated TEM from either compartment promote angiogenesis, which indicates that this population is not dependent on tumor derived factors for function (De Palma et al., 2005). When compared to tumor associated macrophages (TAM), MDSC, endothelial cells, and peritoneal macrophages, the gene profile of TEM and TAM showed the highest level of similarity, and both populations were responsive to Th2 (IL-4) over Th1 (lipopolysaccharide (LPS) + IFN- $\gamma$ ) stimuli (Pucci et al., 2009). TEM, which are primarily Ly6C CD62L', display a similar genetic signature with $\mathrm{Ly}^{-} \mathrm{C}^{-}$ blood monocytes derived from tumor-free mice, which suggests that $\mathrm{Ly}_{6 \mathrm{C}^{-}}$monocytes and TEM may function similarly in steady state and in tumor bearing animals, respectively. In total, these results indicate that function may to a degree be fundamentally encoded in monocyte populations, which can then be modified during exposure to tissue derived factors. This is in contrast and distinct from the concept of macrophage phenotypic plasticity.

\subsection{Murine cancer models}

The dynamic quality attached to mononuclear phagocyte biology systemically is evident in the tumor microenvironment as well. Solid tumors have been characterized as developing organs, consisting of a transformed epithelial component intermixed with non-transformed stromal elements. Models provide a controlled means to study the contribution of individual factors to the formation, growth, persistence, and metastatic potential of tumors (Frese and Tuveson, 2007). Modeling of these interactions commonly occurs through two general methods. The transplant of human cancer cells into immunodeficient mice initiates growth in tissues de novo that can be monitored over a set period of time. This protocol allows flexibility in the selection of tumor cell 
and injection site, and is useful in combination with drug treatment or other manipulations due to the consistency in outgrowth parameters. The signaling pathways in many of the cell lines have been characterized and well documented. Limitations include lack of an adaptive immune system and bypass of the initial events that lead to transformation, however intratumoral interactions can be more directly studied due to the reduced number of contributing factors in the microenvironment. The second type of model involves the genetic manipulation of mice such that tumor formation occurs spontaneously. This system requires no additional manipulation and outgrowth occurs in an otherwise fully intact mouse. Transformation, however, is commonly a result of one oncogenic event, which is abnormal compared to the established double-hit paradigm of tumor formation. Another disadvantage is that the tightly controlled initiation of tumor outgrowth present with transplanted tumor cells is absent in spontaneous models. Tumor formation proceeds at a pace dictated by gene expression and it not necessarily consistent.

Tumor formation in mouse mammary tumor virus polyoma middle T antigen (MMTV-PyVmT) model of breast carcinoma results from the mammary epithelium-targeted expression of the potent oncogene PyVmT (Guy et al., 1992). Expression of the oncogene and subsequent cell membrane incorporation leads to the recruitment and activation of several kinases including Src, mitogen-activated protein kinase (MAPK), and phophatidyl inositol 3 kinase (PI3K) (Novosyadlyy et al., 2009). Hallmarks of this model include $100 \%$ penetrance and disease progression with discernible stages that resemble human breast carcinomas. Metastasis, primarily to the lung, occurs in a high percentage of mice. Additionally, macrophage accumulation correlates with disease progression. Work from Pollard utilizing this model has revealed that the loss of M-CSF significantly delays macrophage accumulation at the tumor and progression to a malignant carcinoma (Lin et al., 2001). They revealed that macrophages regulated an angiogenic switch which increased tumor-associated vasculature and was required for advancement to carcinoma stage. Other groups have utilized the lysozyme M (LysM)-Cre recombinase system in 
the PyVmT model to specifically target vascular endothelial growth factor A (VEGF-A) and hypoxia inducible factor-1 $\alpha$ (HIF-1 $\alpha$ ) for deletion in myeloid lineage cells (Stockmann et al., 2008; Doedens et al., 2010). With the loss of VEGF-A in this lineage, primary tumors grew larger and displayed an accelerated rate of progression to late carcinoma. This occurred along with a reduction in vascularity compared to WT tumors. This result contrasted with the outcome following myeloid-specific deletion of HIF-1 $\alpha$, which led to an increase in tumor apoptosis and a decrease in tumor mass. In Chapter 3 I will present results from our research that addressed the role of focal adhesion kinase (FAK) in myeloid lineage cells in the regulation of breast tumor outgrowth.

\subsection{The focal adhesion kinase (FAK) family of tyrosine kinases}

Cells of the MPS are not sessile. Tracking an individual monocytic cell to terminal fate would show that it traverses three different microenvironments, BM, blood, and peripheral tissue, and breaches two endothelial cell barriers. Experimental evidence has indicated a role for chemokines, cytokines, growth factors, and ECM in the regulation of this process. Intracellular signaling pathways must integrate the complex array of stimuli during this passage and regulate survival, proliferation, differentiation, and migration concurrently.

\subsubsection{Identification and characterization of focal adhesion kinase (FAK)}

Focal adhesion kinase (FAK) was first identified by a monoclonal antibody screen of proteins that displayed enhanced phosphorylation in $\mathrm{pp} 60^{\mathrm{src}}$ transformed embryonic fibroblasts (Kanner et al., 1990). Soon thereafter FAK was cloned and named due to its localization at focal adhesions in cells plated on fibronectin (Schaller et al., 1992). Sequence analysis indicated that FAK was not membrane-associated, did not contain Src-homology 2 (SH2) or SH3 domains, and had prolinerich regions in the C-terminus (Figure 1.6A). In the same year, it was shown that FAK is 
phosphorylated following integrin clustering or adhesion to fibronectin, laminin, and collagen (Kornberg et al., 1992). Carcinoma cells plated on polylysine or held in suspension did not display FAK phosphorylation, indicating that integrin engagement was critical for activation of FAK. Studies in the following two years revealed that the carboxy terminal domain is critical for targeting FAK to focal adhesions and that FAK auto-phosphorylation is necessary for $\mathrm{pp} 60^{\mathrm{src}}$ binding (Hildebrand et al., 1993; Schaller et al., 1994). The characterization of FAK-deficient mice illustrated a role for FAK regulation in fundamental cellular processes (Ilic et al., 1995). These mice displayed general mesodermal defects during development, similar to the phenotype of fibronectin knockout mice, and did not survive beyond embryonic d8.5. In vitro analysis of mesodermal cells from d8.0 embryos indicated that proliferation and differentiation were likely not the cause of the observed abnormalities. Migration of $\mathrm{d} 7.5 \mathrm{FAK}^{-/-}$fibroblast-like cells, however, was severely impaired as they were unable to polarize. Staining for the focal adhesion proteins talin and vinculin indicated that the $\mathrm{FAK}^{-/-}$cells had a reduced number of focal adhesions at the cell periphery compared to wild type (WT) cells but a higher number overall. Thus, over a period of four years, FAK was established as a regulator of cell migration. Experimental evidence in the subsequent years would reveal the mechanisms behind the FAK-regulated alterations in cytoskeletal dynamics required for the establishment of a migratory phenotype, implicate FAK in many prominent intracellular signaling cascades, and suggest a role for FAK in mediating cell proliferation (Parsons et al., 2000; Schaller, 2010) (Figure 1.6A).

\subsubsection{Identification and characterization of proline rich tyrosine kinase 2 (Pyk2)}

Soon after the identification of FAK, three independent groups cloned and named the protein that would ultimately be recognized as proline rich tyrosine kinase 2 (PYK2). These initial studies are very informative in the context of where we now place Pyk2, both with respect to expression and in regulation of cell activity. In one report, the novel protein was identified as related adhesion 
Figure 1.6 The non-receptor tyrosine kinases FAK and Pyk2 display a high degree of sequence homology and mediate signaling through cell-surface receptors. (A) FAK is segregated into an amino terminal FERM domain, central catalytic domain (CAT), and carboxy terminal focal adhesion targeting domain (FAT). Auto-phosphorylation on tyrosine (Y) 397 generates a binding site for the SH2 domain of Src. The FAT domain harbors proline rich regions that can serve as docking sites for SH3 domain-containing proteins such as Cas. Phosphorylation on Y925 permits binding of Grb2. Activation of FAK can occur through various mechanisms, including those elicited through chemokine, integrin, and growth factor engagement. (B) Pyk2 shares high sequence homology with FAK. Src binding to Pyk2 can occur following autophosphorylation on Y402. Pyk2 mediates signaling through various receptor families as well. Figure adapted from Parsons, 2003; Gelman, 2003; Schaller, 2010. 
A.
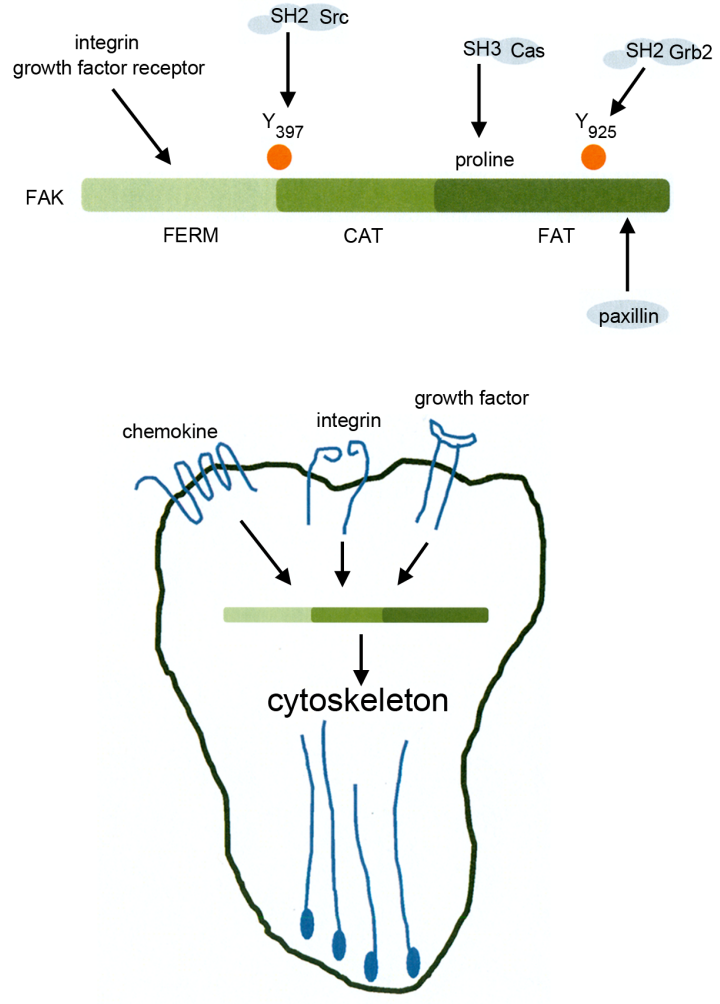

B.
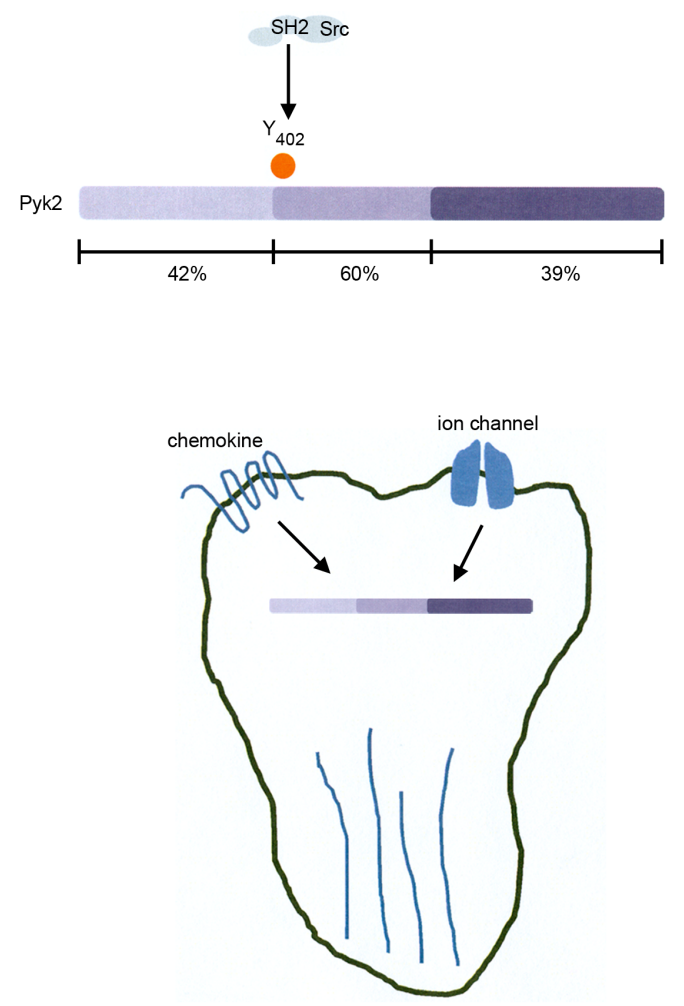
focal tyrosine kinase (RAFTK) given the high degree of sequence identity to FAK. RAFTK was cloned in this study from megakaryotic cells and phosphorylated in a megakaryoblastic leukemia cell line upon thrombin treatment (Avraham et al., 1995). Northern blot analysis of human fetal and adult tissues revealed a more restricted gene expression profile in the former, prompting the authors to conjecture that RAFTK appeared to be developmentally up-regulated. Regions of the brain displayed a comparatively high degree of expression. RAFTK and FAK transcript was detected at equivalent levels in human $\mathrm{CD}_{3} 4^{+}$bone marrow progenitor cells. In another study, this novel protein was designated as Pyk2, given its similarity to proline-rich tyrosine kinase 1 (Pyk1), which was named due to the proline-rich sequence that promoted binding to the SH3 domain of Grb2 (Lev et al., 1995). Treatment of PC12 cells isolated from a pheochromocytoma of the rat adrenal medulla with agonists that activated nicotinic acetylcholine receptors resulted in the rapid phosphorylation of Pyk2. This response was absent with the addition of a calcium chelator, indicating that the phosphorylation of Pyk2 was dependent upon $\mathrm{Ca}^{+}$. It was also revealed that activation of G-protein coupled receptors by bradykinin induced rapid Pyk2 phosphorylation. A consequence of G-protein coupled receptor activation is the release of intracellular calcium stores due to the activity of PKC. The presence of chelators was inconsequential in the activation of Pyk2 if bradykinin, which increases intracellular $\mathrm{Ca}^{+}$levels, was applied. This showed that cytosolic calcium was the critical regulatory factor. Pyk2 could in turn phosphorylate the $\mathrm{K}^{+}$channel Kv1.2, which resulted in the inhibition of channel current. Like FAK, Pyk2 was shown to directly interact with Grb2, and when overexpressed, displayed a dosedependent effect on increased MAPK activity. These results suggested that Pyk2 linked G-protein coupled receptors and calcium influx with activation of the MAPK pathway. In the third study, a novel protein was cloned from adult rat brain that exhibited a high degree of sequence identity to FAK (Sasaki et al., 1995). Named cell adhesion kinase $\beta(\mathrm{CAK} \beta)$, it did not exhibit the same subcellular localization pattern as FAK in COS-7 fibroblasts, as it was found primarily at cell-cell 
junctions rather than in focal adhesions. Additionally, unlike FAK, tyrosine phosphorylation of CAK $\beta$ in $3 Y 1$ fibroblasts did not increase following adhesion on fibronectin.

\subsubsection{FAK, Pyk2, and MPS origins}

Phylogenetic analysis has revealed that putative $F A K$ sequences exist in animals from hydra to humans and that $F A K$ is highly conserved across species (Corsi et al., 2006). Through sequence alignment in 16 species at multiple sites across the full length of the $F A K$ and $P y k 2$, it was revealed that a gene duplication event in deuterosomes subsequent to the echinoderm embranchment likely resulted in $P y k 2$. Thus $P y k 2$ appears to be present only in vertebrates. Comparative analyses suggest that $F A K$ genetic sequences in vertebrates and the common ancestor have been highly conserved, whereas $P y k 2$ appears to have been under less evolutionary constraint. Noted parallels exist when the phylogenies of FAK family kinases and the MPS are overlaid. Macrophage-like cells exist in all multicellular organisms, and their presence preceded monocytes (Takahashi 2000). It was not until primitive vertebrates evolved that monocytes came into existence, as they are not found in the blood of invertebrate animals. This suggests that the emergence of monocytes and macrophages was coincident with Pyk2 and FAK, respectively, and that perhaps the selective pressure that resulted in the generation of monocytes also drove the gene duplication event that resulted in Pyk2. This may indicate that Pyk2 regulates essential functions associated with monocyte biology.

\subsubsection{Functional relationship of FAK and Pyk2}

As the two exclusive members of the FAK family of non-receptor tyrosine kinases, Pyk2 and FAK have many similarities (Parsons et al. 2000; Gelman, 2003; Avraham et al., 2000). They share a high degree of amino acid sequence homology and protein domain structure. Autophosphorylation of tyrosine 397 and 402 in FAK and Pyk2, respectively, leads to the recruitment of Src family kinases (Figure 1.6B). Proline rich motifs in the carboxy terminal of both proteins 
bind SH3 domain-containing proteins. In broad perspective, both molecules are equipped with the necessary tools to localize in a regulated fashion to subcellular regions and influence cell activity through phosphorylation events. Significant differences between FAK and Pyk2 do exist, however. Most notably, the germline deletion of Pyk2 does not lead to embryonic lethality (Okigaki et al., 2003). In fact, no gross abnormalities were seen in these mice. In addition, Pyk2 is expressed predominantly in the nervous system and hematopoietic cells, whereas FAK expression is more universal. Given the similarities in protein structure, one might expect that FAK and Pyk2 could compensate for one another in cells that express both proteins. There is evidence that this occurs, although examples exist indicating that FAK and Pyk2 maintain unique functions. Using the CreLoxP system to induce the targeted deletion of FAK in endothelial cells (EC), it was shown that the loss of FAK resulted in multiple vascular abnormalities during embryogenesis (Shen et al., 2005). Isolated EC infected with adenovirus encoding Cre recombinase induced FAK (CFKO) deletion and were defective in forming capillary tubules on Matrigel. The CFKO displayed increased apoptosis, decreased survival, and reduced migration in response to fibronectin (FN) and vascular endothelial growth factor (VEGF) in a wound closure assay. Phosphorylation of paxillin, c-Jun $\mathrm{N}$ terminal kinase (JNK), and extra cellular signal regulated kinase (ERK1/2) was also reduced in CFKO, however Pyk2 expression was unchanged in these cells. These results indicate that Pyk2 cannot compensate for the loss of FAK in endothelial cells during embryonic development. A study published soon thereafter utilized a similar method to delete FAK and again showed that Pyk2 did not compensate for the loss of FAK in EC (Braren et al., 2006). FAK depletion in EC led to a loss in directional migration on FN, compromised cell spreading on FN and laminin, and the appearance of large, aggregating, abnormal focal adhesions. Severe vascular defects were also observed in vivo. These results conflicted with those from a study that used an inducible system to target EC FAK deletion in adult mice, where the loss of FAK resulted in increased Pyk2 expression and compensation during angiogenesis (Weis et al., 2008). The authors concluded Pyk2 signaling is suppressed in 
EC when FAK is present. It is difficult to reconcile such divergent results regarding the relationship of FAK and Pyk2 in EC. However, FAK was deleted by different methods, and the EC were studied at different stages of development. It is possible that EC utilize different regulatory pathways in the transition from embryo to adult. This is supported by the observation that $\mathrm{FAK}^{-/}$embryonic fibroblasts showed elevated Pyk2 expression as well when compared to fibroblasts from $\mathrm{FAK}^{+/+}$mice (Sieg et al., 1998). Pyk2 could mediate FN-induced ERK activation in $\mathrm{FAK}^{-/-}$fibroblasts, but the normal perinuclear localization of Pyk2 remained in $\mathrm{FAK}^{-/-}$ fibroblasts plated on FN. Moreover, overexpression of Pyk2 in $\mathrm{FAK}^{-/-}$fibroblasts could not rescue the migratory defect of these cells to the extent observed when FAK was re-expressed in these cells. Results from a study in rat smooth muscle cells (RSMC) further highlight divergent regulatory rolls for FAK and Pyk2 (Brinson et al., 1998). Treatment of serum-staved RSMC with either angiotensin II (Ang. II) or platelet-derived growth factor (PDGF) resulted in a significant increase in Pyk2, but not FAK, phosphorylation. This was dependent upon the levels of intracellular calcium, presumably due to the activation of the Ang. II receptor AT1 and PDGF receptor. Another study showed that FAK co-localizes with vinculin at focal adhesions in rat aortic smooth muscle cells plated on FN, whereas Pyk2 maintains a more diffuse and profile, suggesting that FAK and Pyk2 performed divergent functions under these conditions (Zheng et al., 1998).

The studies referenced above highlight the importance of discerning the normal expression patterns of FAK and Pyk2 protein in tissues and cells. FAK is more globally expressed, and in some experimental conditions, the loss of FAK results in the upregulation of Pyk2. There is no evidence to my knowledge of FAK upregulation following the loss of Pyk2. A number of early studies show that FAK is not expressed in monocytes (Lin et al., 1994; Li et al., 1998; Park et al., 2008; De Nichilo and Yamada, 1996; Kedzierska et al., 2001). In most cases, the monocytes utilized in these experiments were derived from peripheral blood and cultured for a short duration 
in growth factors. A potential explanation for the earlier reports suggesting that FAK is not expressed in monocytes is that the analysis was performed on too broad of a population, as there are clearly subsets of monocytes that do express FAK. In Chapter 2, I will present evidence indicating that both FAK and Pyk2 expression is regulated during monocyte differentiation, and that defined subsets of monocytes do in fact express FAK. In mature macrophages, FAK and Pyk2 are expressed and regulate migration and cytoskeletal dynamics (Owen et al., 2007; Okigaki et al., 2003). Deletion of either protein compromises migration in vitro and in vivo. Deletion of both does not result in a further migratory defect toward M-CSF, suggesting that FAK and Pyk2 play a similar role in signaling through the M-CSF receptor. The response to other chemoattractants under this scenario has not been addressed.

\subsection{Goals of this dissertation}

The MPS has been studied extensively during steady state and disease and from this hallmarks have been established regarding the linear progression of differentiation, phenotypic plasticity, migratory capabilities, and effector functions of monocytic cell populations. These fundamental properties have stemmed from observations made at the molecular level and systemically. The volume of literature attached to mononuclear phagocyte research is telling, and is suggestive of their perceived and verifiable importance in the regulation of human biological functions.

However, amidst this vast sum of acquired knowledge rests a field that continues to evolve. Manuscripts generated within the last three years have resulted in a need to reevaluate the derivation of tissue-resident macrophage populations. Arguments made in these studies suggest that under steady state, the MPS contributes minimally to this pool of cells. The majority of data also indicates that monocytes that are recruited into peripheral tissues in response to local production of chemotactic factors differentiate into effector macrophages or dendritic cells. Yet 
recent evidence suggests that monocytes can persist in tissues in an undifferentiated state. The finding that M-CSF may direct myeloid lineage commitment of hematopoietic progenitor cells in the BM through activation of the transcription factor PU.1 challenges the notion that this process occurs by chance. Additionally, the recent identification of IL-34, an alternative M-CSFR ligand, suggests that unappreciated factors exist that could have significant implications in MPS differentiation and behavior.

With these discoveries comes the need to better understand the regulatory mechanisms supporting MPS phenotype. The biological complexity of mononuclear phagocytes hints at a similarly complex network of regulation. Data indicates this to be true. Two fundamental properties govern function and offer a degree of simplicity on a broader scope. These cells have to move and they must be able to integrate an array of extracellular stimuli. Studies in the FAK field have been conducted primarily outside the domain of the MPS and have instead addressed migration and proliferation of mesenchymal and cancer cells. The few studies investigating FAK function in the hematopoietic system have focused on leukemias and less differentiated cells. This is likely due in part to the reported discrepancies in FAK expression in the MPS. Thus, to this point there has existed a pronounced gap in understanding how FAK family kinases contributes to MPS, particularly in undifferentiated lineage-committed monocytic cells. The research presented in this thesis addresses this question from two directions. First, it set out to determine the expression profile of FAK and Pyk2 in monocytes at different stages of differentiation, and to use this information to understand how FAK/Pyk2 regulate differentiation of cells within the MPS (Chapter 2). We demonstrate that FAK and Pyk2 expression increase as monocyte populations differentiate and that Pyk2 is expressed prior to FAK in these monocytes. In response to specific factors, Pyk2 functions to dampen differentiation, whereas FAK acts as an accelerator in this process. Given the established functional activities of FAK presented in this introductory chapter, the second goal of this research was to investigate the role of FAK in a tumor setting (Chapter 3). 
While data from these studies are preliminary, they suggest that FAK in myeloid lineage cells integrates stimuli in a tumor setting that contribute to breast tumor outgrowth (Figure 1.7). 
Figure 1.7 Potential FAK regulated interactions between the primary tumor microenvironment and macrophages. Soluble factors and extracellular matrix present in the tumor microenvironment can bind to cognate receptors on macrophages and activate FAK, leading to downstream activation of signaling cascades and cytoskeletal rearrangement. FAKregulated pathways in macrophages alter macrophage function, thus generating a feedback loop that influences tumor progression. 
Primary Tumor

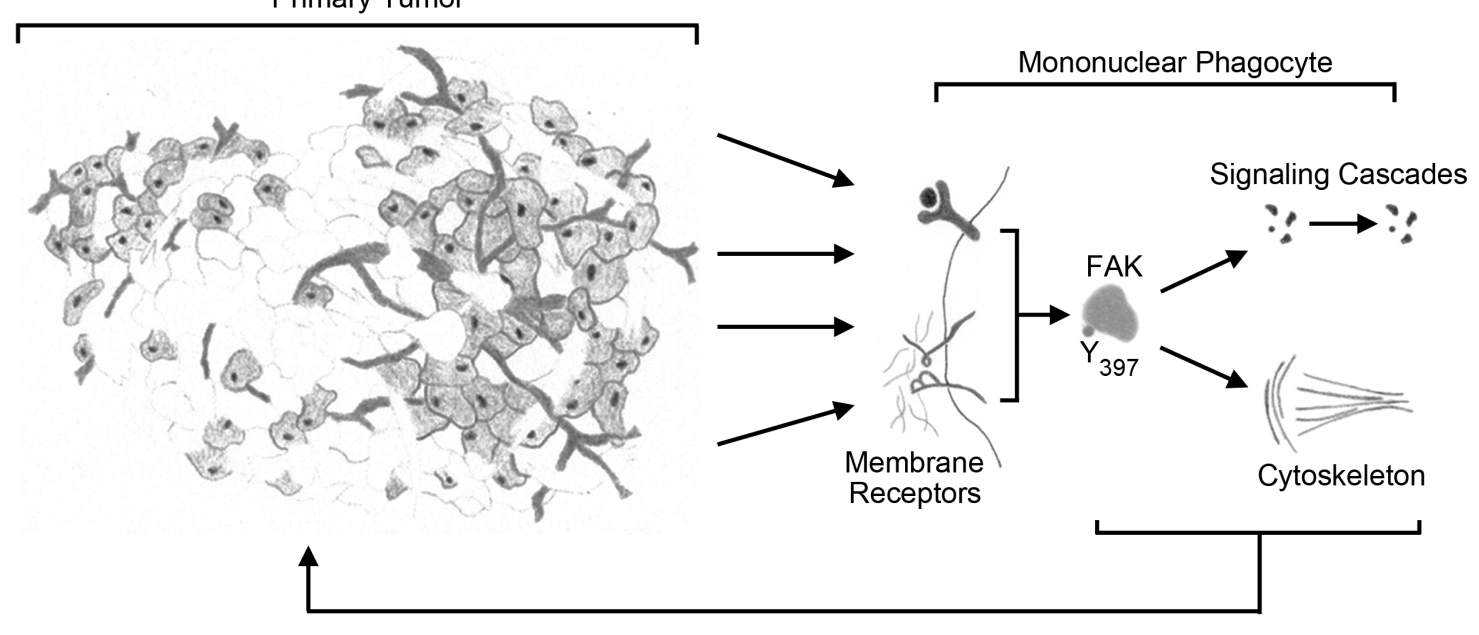




\section{Chapter 2}

The Regulated Expression of FAK and Pyk2 Modulates Monocyte Differentiation

\subsection{Introduction}

Monocytes and macrophages are dispersed throughout adult murine and human tissues and are essential contributors of protective immunity and homeostasis (Gordon and Taylor, 2005). Cells of this lineage originate from tissue resident populations established during embryonic development and in the bone marrow, where self-renewing hematopoietic stem cells (HSCs) differentiate and acquire lineage commitment through the tightly regulated expression of transcription factors (Schulz et al., 2013; Valledor et al., 1998). Subsequent egress of bone marrow monocytes to the vasculature results in two populations of blood monocytes that are classified based on the relative surface expression of Ly6C (Serbina and Pamer, 2006; Geissmann et al., 2003). Non-terminally differentiated Ly6 $\mathrm{C}^{\text {high }}$ monocytes localize to activated regions on the vascular endothelium in response to the expression of factors that promote chemotaxis and adhesion (Kamei and Carman, 2010). A sequential process of stronger monocyte adhesion and directed movement leads to the transmigration of stably bound cells across the endothelial cell layer, where migration through the subendothelial matrix and exposure to soluble factors within the tissue microenvironment provide the stimuli for macrophage terminal differentiation and effector function (Schenkel et al., 2004; Muller and Randolph 1999; Nahrendorf et al., 2007; Lin et al., 2009; Zigmond et al., 2012). The second population of cells, Ly6C ${ }^{\text {low }}$ monocytes, attach and migrate along the endothelium in a regulated process and function in a patrolling and scavenging capacity (Auffray et al., 2007). Experimental evidence suggests that egress of Ly $6 \mathrm{C}^{\text {low }}$ monocytes from the vasculature is significantly more limited compared to the efficient extravasation exhibited by Ly6 $\mathrm{C}^{\text {high }}$ monocytes during inflammation. 
Focal adhesion kinase (FAK) is a highly conserved, non-receptor tyrosine kinase that regulates outside-to-in signaling through integrin engagement and growth factor signaling (Parsons et al., 2000; Sieg et al., 2000). The ubiquitous expression of FAK combined with the embryonic lethality of FAK knockout mice are suggestive of a prominent role for FAK in fundamental cellular processes (Ilic et al., 1995). Proline-rich tyrosine kinase 2 (Pyk2), a FAK family member that shares significant amino acid sequence homology, is expressed in a more limited scope and can be activated through various mechanisms, including increased intracellular calcium and antigen receptor engagement on lymphocytes (Avraham et al., 2000). Pyk2 expression is relatively high in neuronal and hematopoietic cells. Experimental evidence indicates that FAK and Pyk2 can compensate for one another under certain conditions, although there are many examples where this does not occur (Schaller, 2010; Shen et al., 2005; Braren et al., 2006; Owen et al., 2007; Okigaki et al., 2003).

Previous work has addressed the role of FAK and Pyk2 in the early stages of monocyte differentiation and in mature macrophage function. Loss of FAK in $\mathrm{Sca}^{+}$progenitor cells and hematopoietic progenitor cells that had been treated with the colony-inducing stem cell factor (SCF) resulted in reduced migration to CXCL12 (SDF-1 $\alpha$ ) but did not impact the ability of these cells to proliferate and form colonies (Glodek et al., 2007). Additional studies have indicated that FAK and Pyk2 play critical roles in the regulation of macrophage migration to multiple chemokines, growth factors, and inflammatory signals in vivo (Owen et al., 2007; Okigaki et al., 2003). Another study, however, demonstrated that the loss of FAK in bone marrow (BM) cells results in reduced colony formation in response to treatment with several factors, including MCSF, IL-3+SCF, and G-CSF+IL-3, suggesting that FAK regulates proliferation, differentiation, and/or migration in a cytokine/growth factor dependent manner (Vemula et al., 2010).

While these studies have addressed FAK and Pyk2 function in early monocyte/macrophage differentiation, proliferation and migration, very little additional attention has been given to the 
role of FAK and Pyk2 in regulating the differentiation and migration of lineage-committed $\mathrm{CD}_{1} 1 \mathrm{~b}^{+}$monocyte populations. Found primarily in the BM, blood, and spleen, these cells have the capacity to exit the bone marrow, survive and differentiate in circulation, and migrate across the endothelial cell layer. Simultaneously, responses to stimuli that promote terminal differentiation and maturation are dampened until these cells establish residency in peripheral tissues. It has been shown that FAK and Pyk2 are expressed to variable levels during the early and later stages of monocyte differentiation, but their expression and function in non-terminally differentiated $\mathrm{CD} 11 \mathrm{~b}^{+}$monocytes, in which integrin and chemokine signaling play a prominent role, has not been addressed (Kume et al., 1997; Glodek et al., 2007; Owen et al., 2007; Okigaki et al., 2003).

In this study we demonstrate that FAK and Pyk2 expression increase as monocyte populations differentiate and that Pyk2 is expressed prior to FAK in these monocytes. Analysis of Ly6C expression with the differentiation/maturation marker F4/80 indicates that the expression of FAK, but not Pyk2, increases as monocytes lose Ly6C and that Pyk2 expression is concomitant with a gain in F4/80. In response to GM-CSF + IL-6, our data indicate that Pyk2 either suppresses differentiation to fully mature $\mathrm{Ly} 6 \mathrm{C}^{-} \mathrm{F} 4 / 80^{+}$macrophages and/or regulates the survival of Ly6C+F4/80+ monocytes; thus, in the absence of Pyk2, mature Ly6C ${ }^{-} \mathrm{F} 4 / 80^{+}$cells accumulate coincident with a decrease in the number of precursor $\mathrm{Ly}_{6 \mathrm{C}}^{+} \mathrm{F} 4 / 80^{+}$cells. FAK, in contrast, promotes the differentiation of $\mathrm{Ly} 6 \mathrm{C}^{+} \mathrm{F} 4 / 80^{+}$cells and/or enhances the survival of Ly6C-F4/80+ macrophages. Thus, with the loss of FAK, we observed a modest but significant decrease in the proportion of $\mathrm{Ly} 6 \mathrm{C}^{-} \mathrm{F} 4 / 80^{+}$macrophages in response to M-CSF treatment. Taken together, our data indicate that 1) FAK and Pyk2 have opposing roles during monocyte differentiation, with FAK promoting and Pyk2 providing a brake on differentiation and/or 2) FAK and Pyk2 regulate the survival of Ly6C-F4/80+ and Ly6C+F4/80+ monocytes, respectively. 


\subsection{Materials and Methods}

2.2.1 Mouse Models. A description of the $\mathrm{FAK}^{\Delta \text { myeloid }}$ and Pyk $2^{-/-}$mouse models can be found in previously published manuscripts (Owen et al., 2007; Yu et al., 2005).

2.2.2 Harvest and preparation of cell suspensions from mouse tissues. Mice were euthanized with carbon dioxide and tissues were harvested in the following order. Blood (500-700 $\mu 1 /$ mouse) was drawn through cardiac puncture, placed in $1 \mathrm{ml} 5 \mathrm{mM}$ EDTA/HBSS $(-\mathrm{Mg},-\mathrm{Ca})$, washed in HBSS, and washed in MACS buffer $(0.5 \% \mathrm{BSA} / 250 \mathrm{mM}$ EDTA/PBS). The spleen was placed in 10\% FBS/DMEM, homogenized between glass microscope slides, washed in MACS buffer, and filtered through a $30 \mu \mathrm{m}$ filter. BM was flushed from the femurs and tibias with MACS buffer, washed, and filtered through a $30 \mu \mathrm{m}$ filter. To remove erythrocytes, the tissues were incubated in ACK lysis buffer $\left(155 \mathrm{mMNH}_{4} \mathrm{Cl} / 10 \mathrm{mM} \mathrm{KHCO}_{3} / 0.1 \mathrm{mM} \mathrm{Na} \mathrm{KDTA}_{2} 2 \mathrm{H}_{2} \mathrm{O} / \mathrm{H}_{2} \mathrm{O}\right)$ for $5 \mathrm{~min}$. at room temperature, quenched with complete media, and washed in MACS buffer.

\subsubsection{Flow cytometry to distinguish $\operatorname{Lin}^{-} \mathrm{CD} 11 \mathrm{~b}^{+}$monocyte populations. Lin ${ }^{-} \mathrm{CD} 11 \mathrm{~b}^{+}$} monocyte populations were isolated from murine BM, blood, and spleen. Aliquots containing $0.2 \times 10^{6}$ cells from each tissue were transferred to a 96 well plate and incubated with the FC blocking antibody CD16/32 (eBioscience) [1:100] for $10 \mathrm{~min}$. The cells were then incubated with the following antibody panel for 25 minutes on ice: $\alpha$-F4/80AF488 (AbD serotec) [1:100], $\alpha-$ CD11bPE (Invitrogen) [1:400], $\alpha$-Ly6CPerCP/Cy5.5 (BioLegend) [1:400], $\alpha$-Ly6GAPC (Biolegend) [1:200], $\alpha$-CD3eAPC (eBioscience) [1:200], $\alpha$-CD19APC (eBioscience) [1:200], $\alpha$ CD49bAPC (eBioscience) [1:200], and live/dead violet (Invitrogen) [1:200]. Samples were stained with fluorescence minus one (FMO) antibody panels at the same time. Following a series of washes, the cells were fixed for 20 minutes in cytofix (BD Biosciences) and resuspended in MACS buffer. All samples were analyzed for fluorescence intensity on the CyAn ADP LX (Beckman Coulter). Analysis was completed using FlowJo software (Tree Star Inc., v.9.2). Lin ${ }^{-}$ 
$\mathrm{CD}_{1} 1 \mathrm{~b}^{+}$monocyte populations were isolated from in vitro derived monocytes/macrophages. Cell cultures were washed 3 times with PBS and adherent cells were collected following treatment with trypsin/EDTA. $0.1 \times 10^{6}$ cells/condition were aliquoted into 96 well plates for staining. Staining and analysis were completed as described above.

2.2.4 Immunomagnetic column separation of cell populations. Lin $^{-} \mathrm{CD} 11 \mathrm{~b}^{+}$monocytes were isolated from mouse BM, blood, and spleen. Tissues were processed and single cell suspensions were prepared as described above. BM (30 × $10^{6}$ cells $)$, blood $\left(3 \times 10^{6}\right.$ cells $)$, and spleen $\left(30 \times 10^{6}\right.$ cells) suspensions were washed in MACS buffer and FC receptors were blocked with $\alpha$-CD16/32 [1:10] for $10 \mathrm{~min}$. The cells were incubated with the following antibody panels for $25 \mathrm{~min}$. on ice to distinguish lineage positive cells $\left(\operatorname{Lin}^{+}\right)$: $\alpha$-Ly6GAPC (BM [1:10]; Blood, spleen [1:20]), $\alpha$ CD3eAPC (BM, blood [1:20]; spleen [1:10]), $\alpha$-CD19APC (BM, blood [1:20]; spleen [1:10]), $\alpha-$ CD49bAPC (BM, blood, spleen [1:10]). The suspensions were washed in 10ml MACS buffer two times before incubation with $\alpha$-APC conjugated magnetic beads (Miltenyi): BM (200 $\mu 1$ MACS + $50 \mu 1$ bead), blood $(80 \mu 1 \mathrm{MACS}+20 \mu 1$ bead $)$, spleen $(200 \mu 1 \mathrm{MACS}+50 \mu 1$ bead $)$ were incubated for $15 \mathrm{~min}$. on ice. The separation was performed over MS magnetic columns following the manufacturer's protocol. The negative fraction (flow-through) was collected, washed, and incubated with $\alpha$-CD11b conjugated beads $(80 \mu 1$ MACS $+20 \mu 1$ beads) for 15 min. on ice. The suspensions were separated by magnetic column and $\mathrm{CD} 11 \mathrm{~b}^{+}$cells were eluted in MACS buffer for further analysis. Lin ${ }^{-L} 6 \mathrm{C}^{\text {high }}$ and $\mathrm{Lin}^{-} \mathrm{Ly} 6 \mathrm{C}^{\text {low }}$ cells were isolated from BM, blood, and spleen. Tissue cell suspensions were generated as described above. $\operatorname{Lin}^{+}$cells from BM $\left(20 \times 10^{6}\right.$ cells), blood $\left(10 \times 10^{6}\right.$ cells $)$, and spleen $\left(20 \times 10^{6}\right.$ cells $)$ were removed from the cell suspensions by positive immunomagnetic column separation as previously described with modification. Incubation with $\alpha$-APC magnetic beads was completed in $160 \mu 1$ MACS $+40 \mu 1$ bead. The Lin ${ }^{-}$ flow through was washed and resuspended in $180 \mu 1 \mathrm{MACS}+20 \mu 1 \alpha-\mathrm{Ly} 6 \mathrm{CAPC} / \mathrm{Cy} 7$ (Biolegend) for $25 \mathrm{~min}$. on ice. The cells were washed two times and resuspended in $80 \mu 1$ MACS $+20 \mu 1 \alpha-$ 
APC bead for $15 \mathrm{~min}$. on ice. The eluate $\left(\operatorname{Lin}^{-} \mathrm{Ly} 6 \mathrm{C}^{\text {high }}\right)$ and flow-through (Lin-Ly6C $\left.{ }^{\text {low }}\right)$ were retained for further analysis. $\mathrm{Lin}^{-} \mathrm{CD} 11 \mathrm{~b}^{+}$monocytes were isolated from day 2 and day $4 \mathrm{M}-\mathrm{CSF}$ treated BM. Adherent cells were washed 3 times with PBS and incubated for 15 min. at $37^{\circ} \mathrm{C}$ with a trypsin/EDTA solution. The cells were quenched with $10 \%$ FBS/DMEM, washed in MACS buffer, and enumerated. Lin $^{+}$cells were removed as described above, with modification. The antibodies were used at the following concentrations: $\alpha$-Ly6GAPC [1:20], $\alpha$-CD3eAPC [1:40], $\alpha$-CD19APC [1:40], $\alpha$-CD49bAPC [1:40]. Following 2 washes, the cells were resuspended in $80 \mu 1 \mathrm{MACS}+20 \mu 1 \alpha$-APC magnetic bead and incubated for $15 \mathrm{~min}$. on ice. The Lin $^{-}$cells (flow-through) were retained following magnetic column separation and $\mathrm{CD}_{1} 1 \mathrm{~b}^{+}$cells from this fraction were selected as described above.

2.2.5 In vitro derived monocyte/macrophage populations from BM. Whole BM was collected as described above. 4-6 $\times 10^{6}$ cells were added to the following media preparations. For GM-CSF + IL-6 conditions, GM-CSF [40ng/ml] (Peprotech) and IL-6 [40ng/ml] (Peprotech) were added to RPMI1640 supplemented with 10\% FBS, 2mM L-glutamine, 10mM HEPES, penicillin/streptomycin, $20 \mu \mathrm{M} \beta$-mercaptoethanol. For M-CSF conditions, 50ml of L929 conditioned media was added to $\alpha$ MEM supplemented with $10 \%$ FBS and penicillin/streptomycin. At 2 or 4 days post-plating the adherent cells were washed 3 times with PBS incubated with trypsin/EDTA for $15 \mathrm{~min}$. at $37^{\circ} \mathrm{C}$, quenched with complete media, washed with MACS buffer, and enumerated for flow cytometry.

2.2.6 Separation of $\mathrm{Ly}^{\mathrm{h}} \mathrm{C}^{\text {high }}$ and $\mathrm{Ly6C}^{\text {low }}$ monocyte populations. Whole bone marrow was collected and cultured as described above. At day 3, adherent cells were collected as described above. Approximately $2 \times 10^{6}$ cells were washed in MACS buffer and incubated with $\alpha$-CD16/32 [1:10] for 10 min. on ice. The cell suspension was incubated with $\alpha$-Ly6GAPC [1:20] for 25 min. on ice followed by 2 washes. Ly $6 \mathrm{G}^{\text {high }}$ cells were removed by incubating the suspension with $\alpha$ APC conjugated magnetic beads $(80 \mu \mathrm{l}$ buffer $+20 \mu \mathrm{l}$ bead) for $15 \mathrm{~min}$. on ice followed by 
magnetic column separation. The flow-through was incubated with $\alpha$-Ly6CAPC/Cy7 [1:20] for 25 min. on ice, washed twice, and incubated with $\alpha$-APC beads $(80 \mu 1$ buffer $+20 \mu 1$ bead) for 15 min. on ice. Following magnetic column separation, the Ly6G-Ly6C $\mathrm{C}^{\text {high }}$ and $\mathrm{Ly} 6 \mathrm{G}^{-} \mathrm{Ly} 6 \mathrm{C}^{\text {low }}$ populations were retained for further analysis.

2.2.7 Immunoblot detection of FAK and Pyk2. Cell suspensions were washed in PBS, pelleted, and incubated in RIPA buffer for 10 min. on ice with intermittent vortex. Debris was pelleted by centrifugation (13,000 rpm x $15 \mathrm{~min}$.) and the supernatants were retained. Protein concentrations were determined by BCA method and equivalent amounts of protein were prepared in sample buffer + SDS. Proteins were separated on an $8 \%$ SDS/PAGE gel, after which they were transferred to nitrocellulose membranes. The membranes were blocked with 5\% milk for 20 min. prior to adding primary antibodies for overnight incubation at $4{ }^{\circ} \mathrm{C} . \alpha$-FAK (Santa Cruz), $\alpha$-Pyk2 (BD Biosciences), $\alpha$-ERK (Cell Signaling), and $\alpha$-AKT (Cell Signaling) were used at $[1: 1000]$ in 5\% milk. The next day, following 3 washes, secondary antibody (FAK, ERK, AKT [1:5000], Pyk2 $[1: 10,000])$ was added for $40 \mathrm{~min}$. at room temperature in $5 \%$ milk. The membranes were washed 5 times, incubated for $2 \mathrm{~min}$. in luminol/peroxidase reagents (Millipore), and exposed to film for detection by enhanced chemical luminescence. Bands in the linear range of detection were quantified by densitometry using Image Quant software. The relative expression of FAK and Pyk2 was determined by normalizing to ERK expression.

2.2.8 In vitro differentiation of FACS isolated $\mathrm{Ly}^{\mathrm{G}} \mathrm{C}^{\text {high }} \mathrm{BM}$ monocytes. A whole $\mathrm{BM}$ cell suspension from 4 WT mice was prepared as described above. Approximately $140 \times 10^{6}$ total cells were collected and aliquoted into 4 staining tubes. The cells were washed and resuspended in $250 \mu 1$ buffer $+\alpha-C D 16 / 32[1: 100]$ for $10 \mathrm{~min}$. on ice. The cells were incubated with the following antibody panel in a final volume of $500 \mu 1$ for 25 min. on ice: $\alpha$-CD11bPE [1:400], $\alpha-$ Ly6CPerCP/Cy5.5 [1:400], $\alpha$-Ly6GAPC [1:200], $\alpha$-CD3eAPC [1:200], $\alpha$-CD19APC [1:200], $\alpha-$ CD49bAPC [1:200], and live/dead violet [1:200]. FMO stains were prepared as controls. 
Following incubation, the cells were washed twice and $\mathrm{Lin}^{-\mathrm{CD}} 11 \mathrm{~b}^{+} \mathrm{Ly} 6 \mathrm{C}^{\text {high }}$ cells were isolated by FACS on the Reflection HAPS 2. Approximately $3 \times 10^{6}$ sorted Ly6C ${ }^{\text {high }}$ cells were aliquoted evenly into three $10 \mathrm{~cm}$ petri dishes containing either the base media (RPMI1640, 10\% FBS, 2mM L-glutamine, 10mM HEPES, penicillin/streptomycin, $20 \mu \mathrm{M} \beta$-mercaptoethanol) (one plate) or base media + GM-CSF [40ng/ml]+ IL-6 [40ng/ml] (duplicate plates). The cells in base media alone was pulsed with BrdU for three hours, harvested, and stained with the following panel for the detection of surface antigens: $\alpha$-Ly6CPerCP/Cy5.5 [1:400], $\alpha$-F4/80APC [1:100], live/dead [1:200]. The cells were subsequently stained for BrdU incorporation following manufacturers protocol (BD pharmingen). At two days post-plating, GM-CSF + IL-6 treated cells were pulsed with BrdU for three hours. Adherent cells were washed twice, harvested following trypsin/EDTA treatment, pooled from the two plates, and stained for surface antigens and BrdU using the identical protocol described for day 0 samples. Flow cytometry was run concurrently for all samples. FlowJo software was used for analysis.

2.2.9 Isolation of in vitro derived monocyte populations by FACS. A single cell suspension of whole BM from 5 WT mice was prepared following the above description. Approximately $6 \mathrm{x}$ $10^{6}$ cells $/ 10 \mathrm{~cm}$ petri dish (24 dishes) were plated in RPMI1640 supplemented with $10 \%$ FBS, 2mM L-glutamine, 10mM HEPES, penicillin/streptomycin, $20 \mu \mathrm{M} \beta$-mercaptoethanol, GM-CSF [40ng/ml], and IL-6 [40ng/ml]. At day 3, adherent cells were washed two times with PBS, incubated with trypsin/EDTA, quenched with complete media, and pooled. Approximately $9 x$ $10^{6}$ cells total were collected. The cells were equally divided into two staining tubes, blocked with $\alpha$-CD16/32 for $10 \mathrm{~min}$. on ice, and incubated for $25 \mathrm{~min}$. on ice with the following antibody panel $(500 \mu 1$ final volume): $\alpha$-F4/80AF488 [1:100], $\alpha$-CD11bPE [1:400], $\alpha$-Ly6CPerCP/Cy5.5 [1:400], $\alpha$-Ly6GAPC [1:200], $\alpha$-CD3eAPC [1:200], $\alpha$-CD19APC [1:200], $\alpha$-CD49bAPC [1:200], and live/dead violet (Invitrogen) [1:200]. FMO stains were prepared for all antibodies. 
Ly6C $\mathrm{C}^{+} \mathrm{F} 4 / 80^{-}, \mathrm{Ly}_{6 \mathrm{C}^{+} \mathrm{F} 4 / 80^{+}, \text {Ly6C }} \mathrm{F} 4 / 80^{+}$monocyte populations were isolated by FACS on the Reflection HAPS 2. The cells were washed in PBS and prepared for immunoblot.

2.2.10 Statistical Analysis. Comparisons between groups were made using the unpaired twotailed student $\mathrm{t}$ test, except for Figure 2.6F and 2.7B (paired two-tailed student $\mathrm{t}$ test). A p value $<$ $.05(*<.05)$ was considered statistically significant.

\subsection{Results}

\subsubsection{FAK and Pyk2 protein expression is elevated in more differentiated CD11 ${ }^{+}$monocyte populations in vivo.}

It has been established that monocytes are prevalent in the bone marrow (BM), blood, and spleen during steady state (Varol et al., 2009). CD11b ( $\alpha \mathrm{M} \beta 2$ ) expression is initiated during myelopoiesis as progenitor cells differentiate to monocytes (Rosmarin et al., 1989; Pahl et al., 1993; Hettinger et al., 2013). To measure FAK and Pyk2 protein expression in CD11b monocytes from these tissues, lineage-positive granulocytes $\left(\mathrm{Ly}_{6} \mathrm{G}^{+}\right), \mathrm{T}$ cells $\left(\mathrm{CD} 3 \mathrm{e}^{+}\right), \mathrm{B}$ cells $\left(\mathrm{CD} 19^{+}\right)$, and NK cells $\left(\mathrm{CD}_{\left.49 \mathrm{~b}^{+}\right)}\right.$were first removed from tissue suspensions by positive immunomagentic column separation. Monocytes were then isolated from the lineage negative (Lin') cell population using $\alpha$-CD11b-conjugated magnetic beads (Figure 2.1). Cell lysates were prepared and immunoblotted for FAK and Pyk2. Both molecules were more highly expressed in monocytes harvested from the spleen compared to bone marrow monocytes (Figure 2.2A). FAK was nearly undetectable in blood monocytes, whereas Pyk2 showed a similar level of expression to the amount present in the spleen. Flow cytometry was performed to determine the differentiation status of the $\operatorname{Lin}^{-} \mathrm{CD} 11 \mathrm{~b}^{+}$monocytes in these tissues (Figure 2.3). Monocyte populations were distinguished using surface expression of Ly6C, which is lost as monocytes differentiate and undergo maturation (Yona and Jung, 2009; Yona et al., 2013). Within the 
Figure 2.1 Isolation of $\mathrm{CD} 11 \mathrm{~b}^{+}$monocytes from murine bone marrow by immunomagnetic column separation. $\mathrm{Lin}^{+}\left(\mathrm{Ly}_{6 \mathrm{G}}{ }^{+}, \mathrm{CD} 3 \mathrm{e}^{+}, \mathrm{CD} 19^{+}, \mathrm{CD}_{9} \mathrm{~b}^{+}\right)$cells were removed from the $\mathrm{BM}$ suspension followed by positive selection of $\mathrm{CD} 11 \mathrm{~b}^{+}$monocytes from the flow-through. Cytospin preparations were made from the Lineage $^{+}$(eluate), Lineage ${ }^{-}$(flow-through), and CD11 $\mathrm{b}^{+}$ (monocytes) populations. Schematic and cytospin images illustrate the progression of isolated cell populations. 


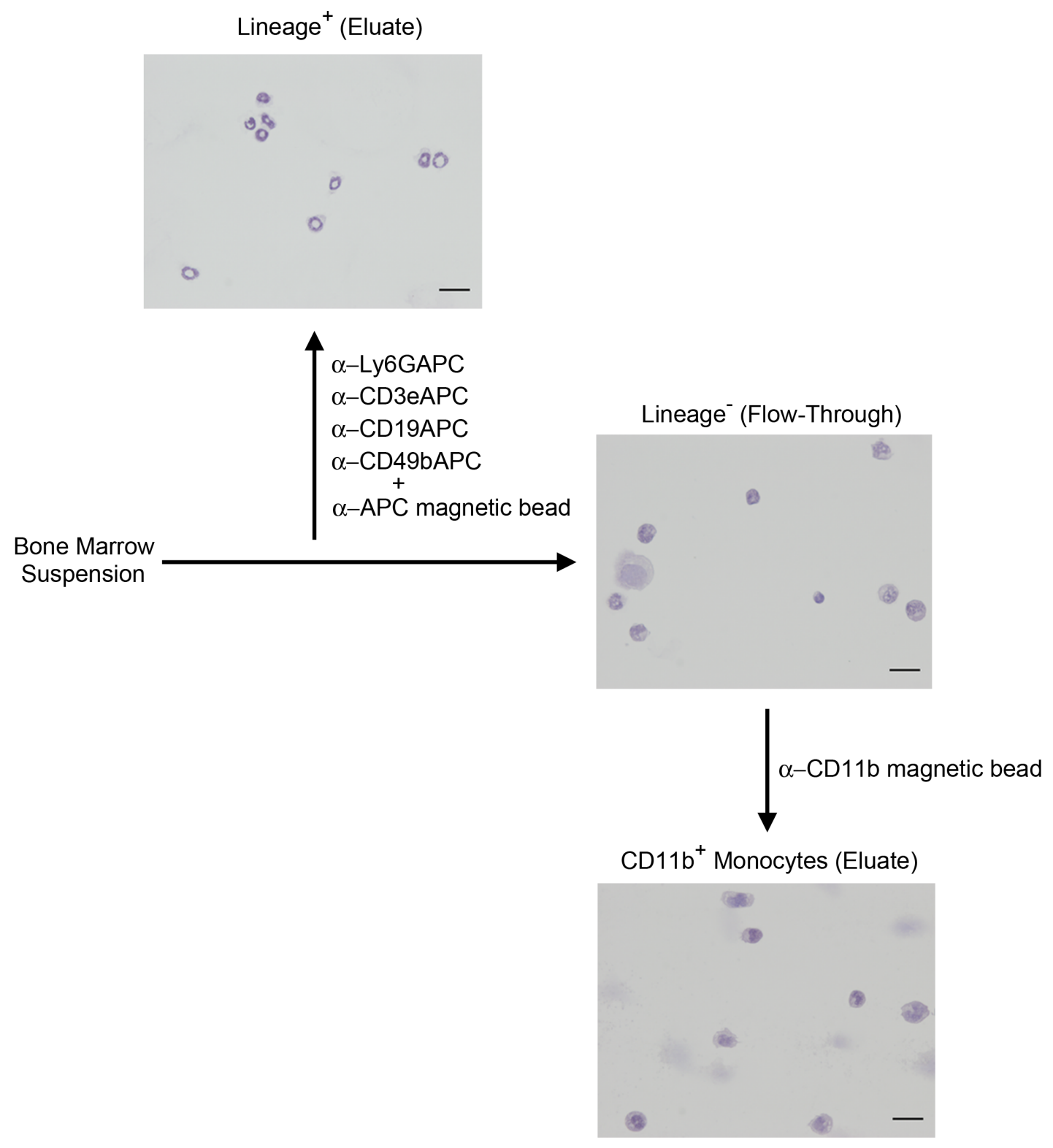


Figure 2.2 FAK and Pyk2 protein expression is regulated in BM, blood, and splenic monocytes. (A) Western blot of lineage (Lin) ${ }^{-}\left(\mathrm{Ly}_{6} \mathrm{G}^{-}, \mathrm{CD}^{-}, \mathrm{CD} 19^{-}, \mathrm{CD} 49 \mathrm{~b}^{-}\right) \mathrm{CD}^{-11 b^{+}}$ monocytes isolated from bone marrow (BM) (lane 1), blood (lane 2), and spleen (lane 3) of wild type (WT) mice. ERK is presented as a loading control. (B) Flow cytometry analysis of Ly6C surface expression on $\operatorname{Lin}^{-} \mathrm{CD} 11 \mathrm{~b}^{+}$monocytes from BM, blood, and spleen. The percentage of Ly6 $\mathrm{C}^{+}$monocytes was determined as events displaying greater fluorescence intensity than fluorescence minus one (FMO) controls out of the total number of $\operatorname{Lin}^{-} \mathrm{CD} 11 \mathrm{~b}^{+}$cells analyzed. Data are representative of seven tissue sets. SEM, ${ }^{*} \mathrm{P}<.05$. (C) Western blot analysis of Lin $^{-}$ $\mathrm{Ly}_{6 \mathrm{C}^{+}}$and $\mathrm{Lin}^{-} \mathrm{Ly}_{6} \mathrm{C}^{-}$cell populations isolated from BM (lanes 1,2), blood (lanes 3,4), and spleen (lanes 5,6) by immunomagnetic column. Cell populations were generated following the removal of $\mathrm{Lin}^{+}$cells from the tissue cell suspensions prior to Ly6C positive selection. ERK is presented as a loading control. (D) Identification of monocyte populations in BM, blood, and spleen by flow cytometry based on Ly6C and F4/80 surface expression. Gates indicate Ly6C ${ }^{+} \mathrm{F} 4 / 80^{-}$and $\mathrm{Ly}^{-} \mathrm{C}^{-}$ $\mathrm{F} 4 / 80^{+}$monocyte populations. FMO staining is represented in the bottom panel set. Bar graph displays the percentage of $\mathrm{Ly} 6 \mathrm{C}^{+} \mathrm{F} 4 / 80^{-}$and $\mathrm{Ly} 6 \mathrm{C}^{-} \mathrm{F} 4 / 80^{+}$monocyte populations. Data are representative of seven tissue sets. SEM, $*=\mathrm{P}<.05$. 
A.

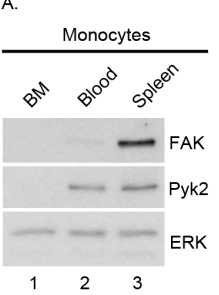

c.

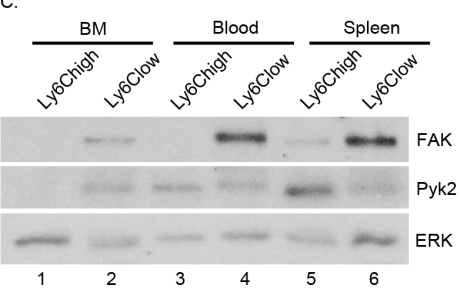

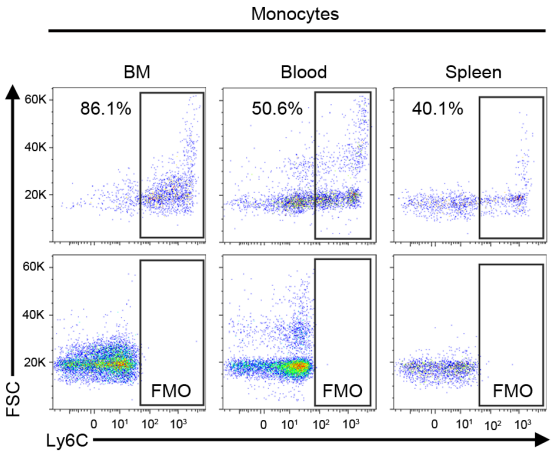
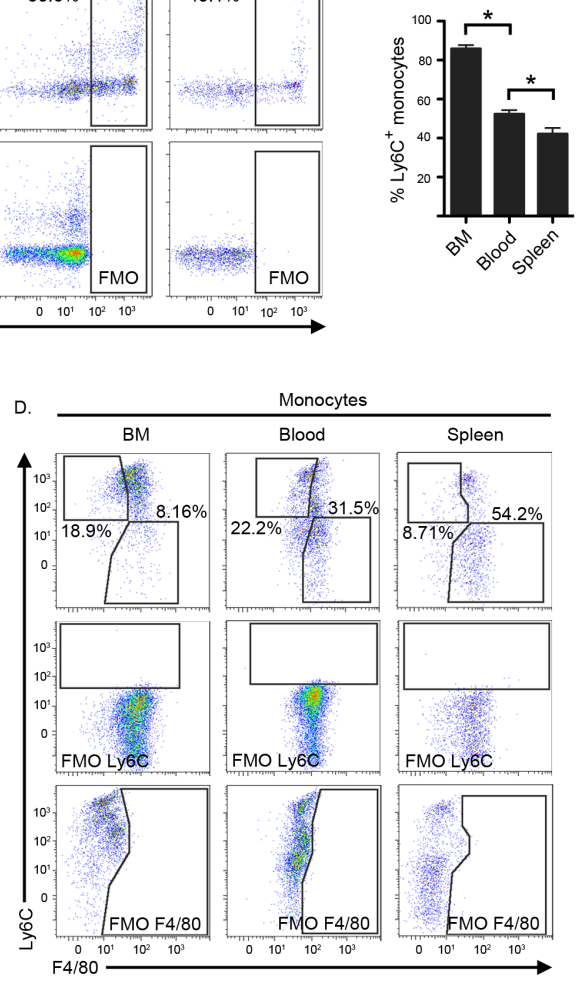

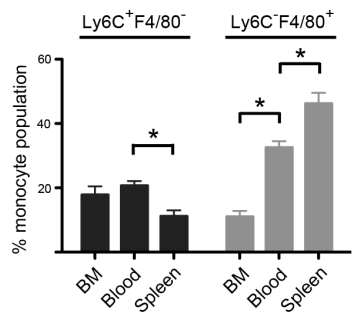


Figure 2.3 Identification of $\operatorname{Lin}^{-} \mathrm{CD}_{11 b^{+}}$monocytes in BM, blood, and spleen by flow cytometry. Flow cytometry dots plots indicate the percentage of cells within a defined gate for BM (A), blood (B), and spleen (C). The titles at the top of each plot indicate the population that is identified by each gate. The gating scheme proceeds from left to right. The Lin ${ }^{-}$cell population

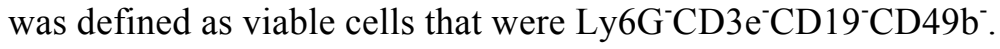


A.

Bone Marrow
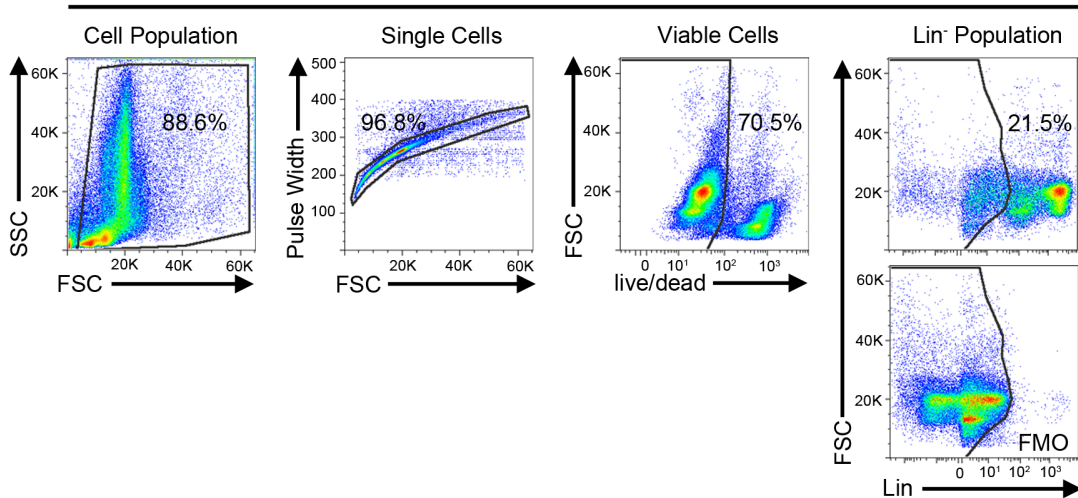

Monocytes
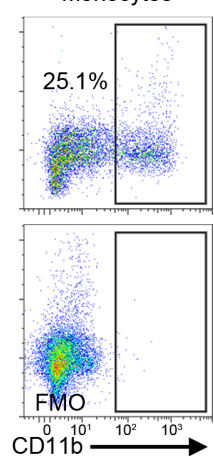

B.

Blood
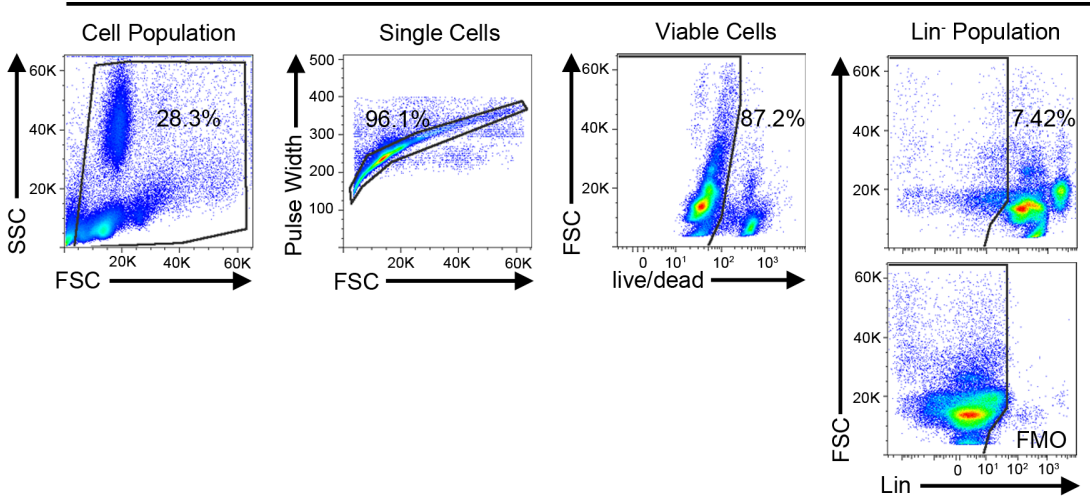

Monocytes
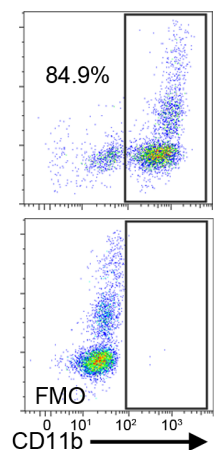

c.

Spleen
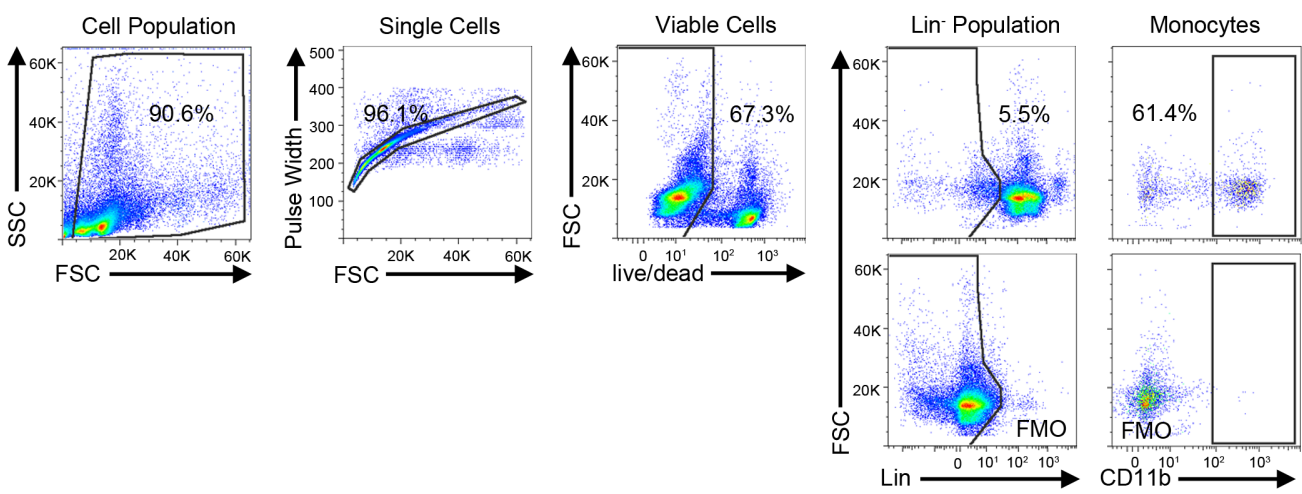
monocyte population, the percentage of cells expressing Ly6C was greatest in the $\mathrm{BM}$, less in the blood, and still less in the spleen (Figure 2.2B). Because FAK and Pyk2 protein expression correlated with an increased proportion of $\mathrm{Ly} 6 \mathrm{C}^{-}$monocytes, we next sought to determine whether expression would track with the loss of Ly6C. Following the removal of Lin $^{+}$cells by magnetic beads, we isolated $\mathrm{Ly} 6 \mathrm{C}^{\text {high }}$ cells from the remaining cell suspension by positive selection. Immunoblot of the lysates generated from this fraction (Lin ${ }^{-L} 6 \mathrm{C}^{\text {high }}$ ) and lysates from the unbound fraction ( $\mathrm{Lin}^{-\mathrm{Ly}} 6 \mathrm{C}^{\mathrm{low}}$ ) indicated that FAK is expressed only in Ly6C ${ }^{\text {low }}$ cells from the $\mathrm{BM}$ and blood, and predominantly in $\mathrm{Ly} 6 \mathrm{C}^{\text {low }}$ splenic cells (Figure 2.2C). Pyk2 expression was equivalent in the $\mathrm{Ly} 6 \mathrm{C}^{\text {high }}$ and $\mathrm{Ly} 6 \mathrm{C}^{\text {low }}$ populations from the blood and appeared slightly elevated in the Ly6 $\mathrm{C}^{\text {high }}$ splenic population. Both FAK and Pyk2 were undetectable in Ly6C ${ }^{\text {high }}$ BM cells.

A second marker of monocyte differentiation that is commonly used to identify mature macrophages is the glycoprotein F4/80 (Austyn and Gordon, 1981; Leenen et al., 1994). The surface expression of F4/80 increases during monocyte differentiation. Given that both Ly6C and F4/80 serve as markers of monocyte differentiation, we next examined monocyte differentiation as a function of Ly6C and F4/80 expression together on BM, blood, and splenic monocytes by flow cytometry. Monocytes expressing the highest level of Ly6C and lowest level of F4/80 $\left(\mathrm{Ly} 6 \mathrm{C}^{+} \mathrm{F} 4 / 80^{-}\right)$, which represent the least differentiated cells, decreased in proportion significantly between the blood and spleen (Figure 2.2D). At the same time, the splenic monocyte population had significantly more of the most differentiated Ly6C $\mathrm{F} 4 / 80^{+}$monocyte population, which is consistent with reports showing that the spleen harbors several distinct macrophage populations (Taylor et al., 2005). Together, these data indicate that 1) FAK and Pyk2 are more highly expressed in $\mathrm{CD}_{1} 1 \mathrm{~b}^{+}$monocyte populations that display a more differentiated phenotype, 2) FAK expression tracks with reduced surface expression of Ly6C, and 3) Pyk2 is expressed earlier in $\mathrm{CD}_{11} \mathrm{~b}^{+}$monocyte differentiation compared to FAK. 


\subsubsection{Pyk2 is required for the maintenance of $\mathrm{Ly}_{6} \mathrm{C}^{+} \mathrm{F} 4 / 80^{+}$mononuclear phagocytes in the BM, blood, and spleen}

Recent fate mapping studies in the BM and blood monocyte compartment have demonstrated that under steady state conditions, $\mathrm{Ly}_{6} \mathrm{C}^{-}$monocytes are derived from $\mathrm{Ly} 6 \mathrm{C}^{+}$monocytes (Yona et al., 2013). This indicates that only the $\mathrm{Ly}_{6 \mathrm{C}^{+}}$population traffics from the BM to blood. Splenic nonterminally differentiated $\mathrm{Ly}_{6} \mathrm{C}^{+}$and $\mathrm{Ly} 6 \mathrm{C}^{-}$monocyte populations are relatively abundant and share many characteristics with blood monocytes (Swirski et al., 2009). We utilized these inherent tissue properties to begin to examine the role of FAK and Pyk2 in monocyte differentiation and/or survival. BM, blood, and splenic monocytes in mice harboring the myeloidspecific deletion of FAK (LysM-Cre FAK ${ }^{\text {fx/fx }} ; F^{-1 \text { myeloid }}$ ) and the germline deletion of Pyk2 $\left(\operatorname{Pyk} 2^{\top}\right)$ ) were analyzed by flow cytometry for Ly6C and F4/80 expression (Owen et al., 2007; Yu et al., 2005). There were no statistical differences in any monocyte population due to the loss of FAK (Figure 2.4). In contrast, there was a significant decrease in the percentage of Ly $6 \mathrm{C}^{+} \mathrm{F} 4 / 80^{+}$monocytes in the $\mathrm{BM}$, blood, and spleen of Pyk $2 \%$ mice, and a concomitant, albeit small, statistically significant increase in the percentage of $\mathrm{Ly} 6 \mathrm{C}^{+} \mathrm{F} 4 / 80^{-}$cells in the BM.

One possibility for the decrease in $\mathrm{Ly} 6 \mathrm{C}^{+} \mathrm{F} 4 / 80^{+}$monocytes in $\mathrm{Pyk} 2^{-} \%^{-}$mice is that these cells may have a defect in proliferation. To determine if there were differences in proliferation between Ly $6 \mathrm{C}^{+}$cells in the $\mathrm{BM}$ of wildtype and $\mathrm{Pyk} 2^{--}$mice, BrdU incorporation was measured by flow cytometry. $\mathrm{BM} \mathrm{CD} 11 \mathrm{~b}^{+} \mathrm{Ly} 6 \mathrm{C}^{+}$monocytes isolated from wildtype, $\mathrm{FAK}^{\Delta \text { myeloid }}$, and $\mathrm{Pyk}^{\%^{-}}$mice all showed the same level of BrdU incorporation, suggesting that FAK and Pyk2 did not regulate proliferation in this population of cells (Figure 2.5). 
Figure 2.4 Loss of Pyk2 results in reduced proportion of $\mathrm{Ly6C}^{+} \mathrm{F4} / \mathrm{80}^{+}$monocytes in blood and spleen. BM (A), blood (B), and spleen (C) monocyte compartments of mice with the conditional deletion of FAK in myeloid lineage cells $\left(\mathrm{FAK}^{\Delta \text { myeloid }}\right)$ and germline deletion of Pyk2 $\left(\mathrm{Pyk}^{-} /\right)$were compared to WT mice. Four $\mathrm{Lin}^{-} \mathrm{CD} 11 \mathrm{~b}^{+}$monocyte populations $\left(\mathrm{Ly}^{-} \mathrm{C}^{+} \mathrm{F} 4 / 80^{-}\right.$, Ly6C $\mathrm{C}^{+} \mathrm{F} 4 / 80^{+}$, Ly6C $\mathrm{F} 4 / 80^{-}$, Ly6C $\mathrm{F} 4 / 80^{+}$) were identified by flow cytometry. Dot plots are representative of $7 \mathrm{WT}, 7 \mathrm{FAK}^{\Delta \text { myeloid }}$, and $4 \mathrm{Pyk}^{2 \%}$ mice. FMO stain for each replicate is identical to FMO panel in Figure 2.2D. Bar graphs display the percentage of each monocyte subset out of the total $\operatorname{Lin}^{-} \mathrm{CD} 11 \mathrm{~b}^{+}$monocyte population. SEM $*=\mathrm{P}<.05$. 

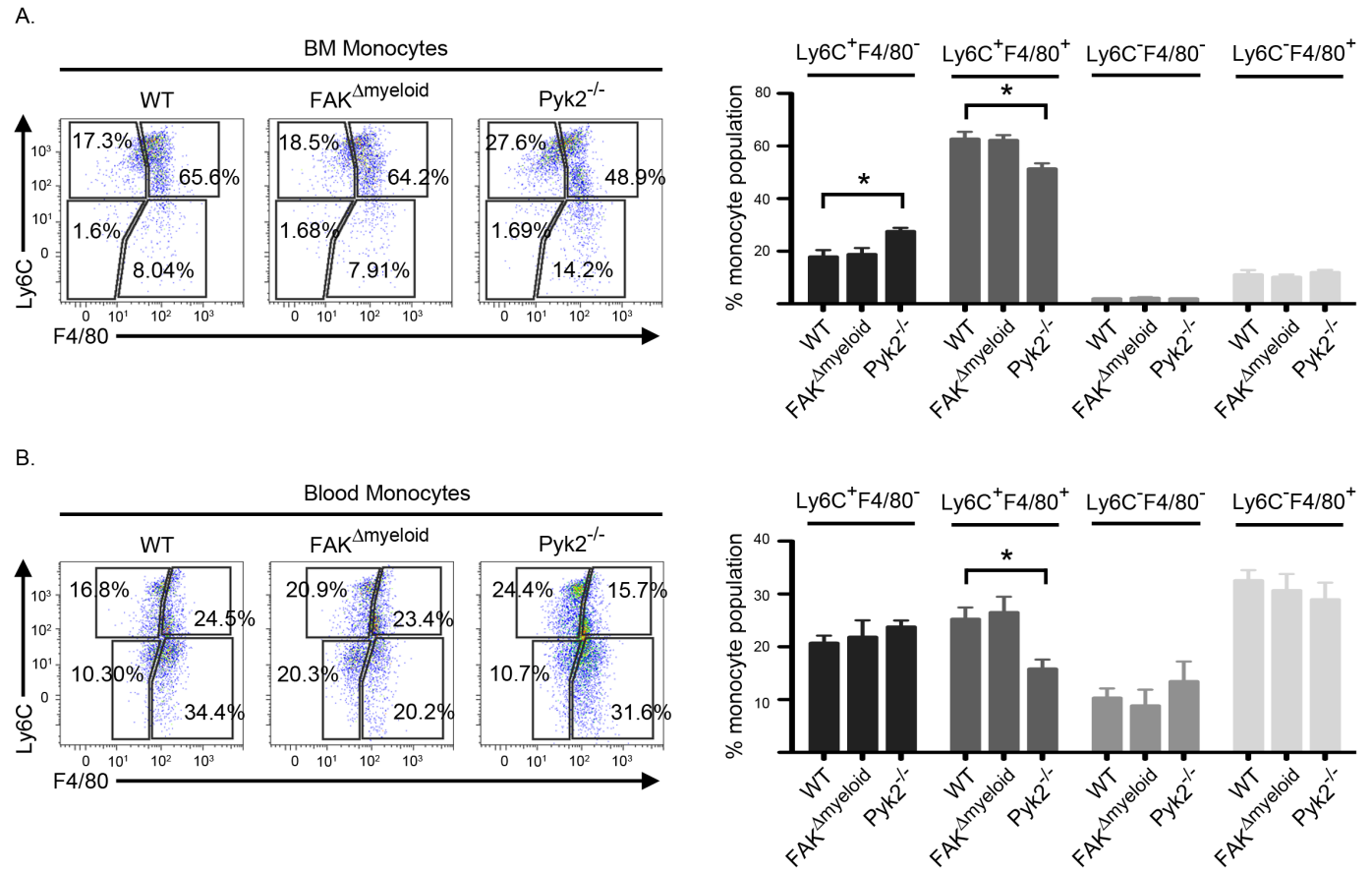

c.

Splenic Monocytes
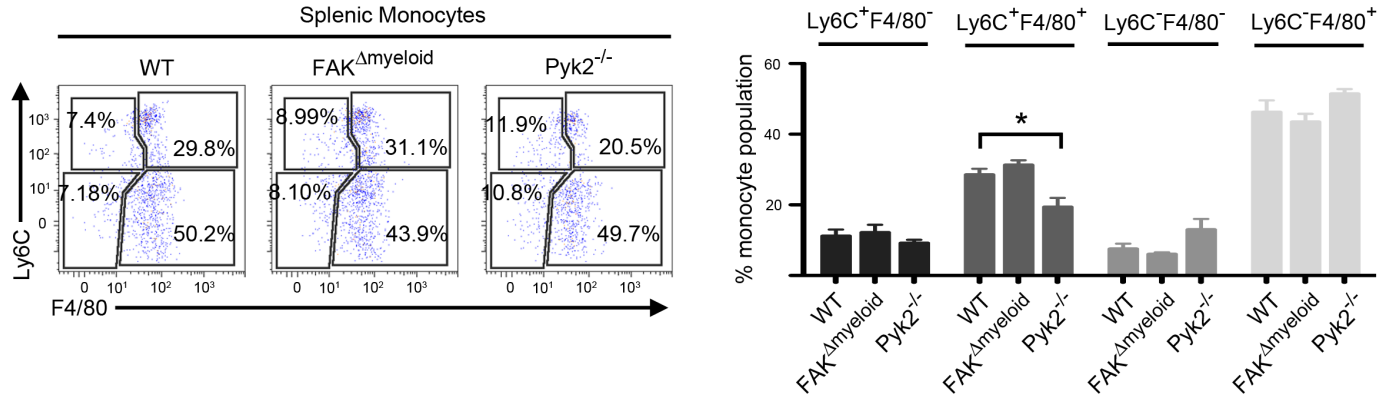
Figure 2.5 Ly6C $^{\text {high }}$ monocytes in the BM display equivalent levels of BrdU incorporation. Whole bone marrow was harvested from WT, $\mathrm{FAK}^{\Delta \text { myeloid }}$, and $\mathrm{Pyk} 2^{-/-}$mice and pulsed with BrdU for three hours, followed by flow cytometry staining to distinguish $\mathrm{BrdU}^{+}$cells. Dot plots indicate the percentage of $\operatorname{Lin}^{-} \mathrm{CD} 11 \mathrm{~b}^{+} \mathrm{Ly} 6 \mathrm{C}^{+} \mathrm{BrdU}^{+}$monocytes. 


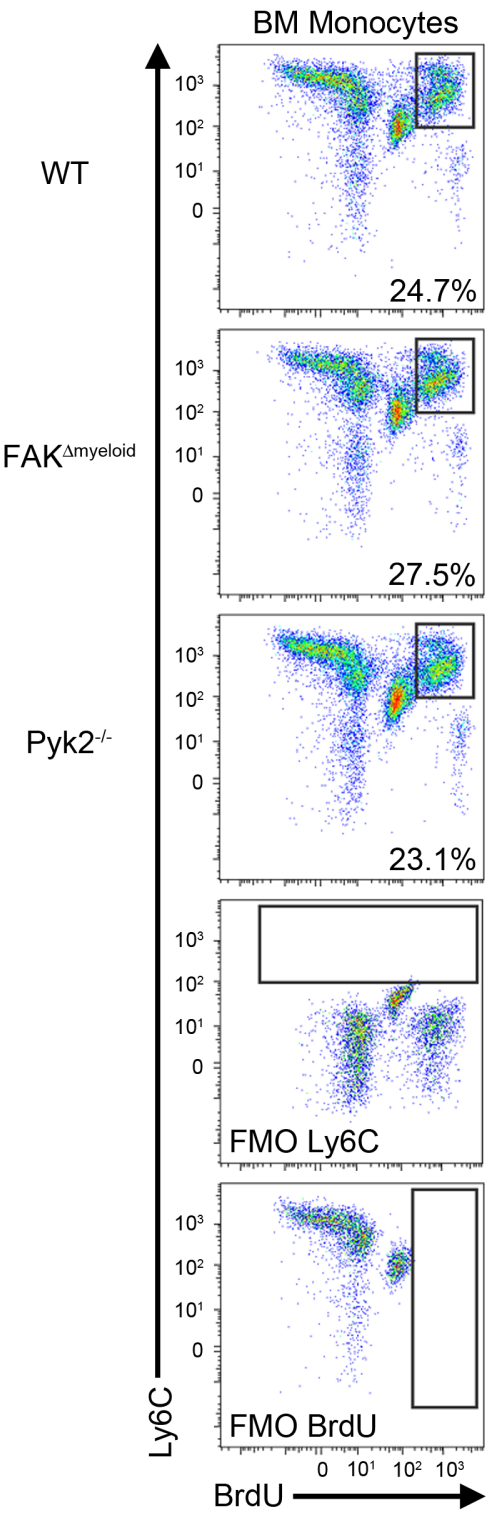




\subsubsection{FAK is expressed predominantly in $\mathrm{Ly}^{6} \mathrm{C}^{\text {low }}$ monocytes}

Our results in vivo led us to more directly define the stage at which FAK and Pyk2 are expressed during monocyte differentiation. Bone marrow differentiation was induced in vitro in the presence of macrophage colony stimulating factor (M-CSF). Over a 4-day period in M-CSF, the cells adopted phenotypic hallmarks of macrophages, as seen by the large size and single nuclei (Figure 2.6A). FAK and Pyk2 expression were elevated in the M-CSF treated monocyte populations (Figure 2.6B) compared to BM monocytes. Similar to what was seen in vivo, Pyk2 expression appeared to reach maximal levels earlier than did FAK (compare day 2 to day 4 samples). During this period, there was a concomitant decrease in the percentage of Ly6 $\mathrm{C}^{+}$monocytes compared to bone marrow monocytes (Figure 2.6C), consistent with the cells becoming more differentiated. The loss of Ly6C together with an increase in the percentage of $\mathrm{F} 4 / 80^{+}$monocytes between day 2 and day 4 (Figure 2.6D) indicated that the $\mathrm{CD}_{1} 1 \mathrm{~b}^{+}$monocytes progressively differentiated over a period of four days in M-CSF.

Because FAK and Pyk2 expression correlated with increased proportions of more differentiated monocytes in both tissues and in M-CSF treated bone marrow cells, we next sought to determine more directly whether differential expression of these proteins was a function of reduced Ly6C expression. Bone marrow cells cultured in vitro with GM-CSF + IL-6 produces several monocyte populations at different stages of differentiation (Marigo et al., 2010). Using magnetic bead selection, we isolated $\mathrm{CD} 11 \mathrm{~b}^{+} \mathrm{Ly} 6 \mathrm{C}^{\text {high }}$ and $\mathrm{CD} 11 \mathrm{~b}^{+} \mathrm{Ly} 6 \mathrm{C}^{\text {low }}$ adherent monocyte populations from BM cells cultured for 3 days in GM-CSF+IL-6 (Figure 2.6E). FAK expression was elevated in the Ly6 $\mathrm{C}^{\text {low }}$ monocytes compared to the Ly6 $\mathrm{C}^{\text {high }}$ population (Figure 2.6F). In contrast, Pyk2 expression was similar in both populations. Histological preparations displayed the presence of significantly more macrophage-like cells in the Ly6 $\mathrm{C}^{\text {low }}$ population (Figure 2.6G). An identical magnetic bead selection protocol was applied to day $3 \mathrm{M}-\mathrm{CSF}$ treated BM and resulted in a similar pattern of FAK and Pyk2 expression in Ly6 $\mathrm{C}^{\text {high }}$ and Ly6C $\mathrm{C}^{\text {low }}$ monocytes (Figure 2.7). 
Figure 2.6 FAK and Pyk2 expression increase during monocyte differentiation in vitro. FAK expression is elevated in monocytes that have reduced Ly6C surface expression. (A) Images display hematoxylin stained cytospin preparations of BM, day 2, and day $4 \mathrm{Lin}^{-} \mathrm{CD} 11 \mathrm{~b}^{+}$ monocytes. (B) Cell lysates of adherent $\operatorname{Lin}^{-C D} 11 \mathrm{~b}^{+}$monocytes after two (d2, lane 2) and four (d4, lane 3) days treatment of whole BM with M-CSF were compared to BM Lin CD11b monocytes (lane 1) by immunoblot. (C) Flow cytometry dot plots display Ly6C surface expression on representative examples of $\mathrm{BM}$, day 2 , and day $4 \mathrm{Lin}^{-} \mathrm{CD} 11 \mathrm{~b}^{+}$monocytes. Bar graph displays the percentage of monocytes that stained positive for Ly6C out of the total monocyte population. (D) F4/80 surface expression was determined by a similar staining method of BM, day two, and day four monocytes. Bar graph depicts the percentage of $\mathrm{F} 4 / 80^{+}$monocytes out of the total $\mathrm{Lin}^{-} \mathrm{CD} 11 \mathrm{~b}^{+}$monocyte population. Data in (C) and (D) are from 7 replicates per condition. SEM, * $=\mathrm{P}<.05$. (E) WT BM was treated in vitro with GM-CSF (40ng/ml) + IL-6 (40ng/ml) for three days. Adherent cells expressing Ly6G were removed by positive magnetic bead selection. The resulting flow-through, consisting of Ly6 $\mathrm{C}^{\text {high }}$ and Ly6Clow monocytes, was separated for $\mathrm{Ly} 6 \mathrm{C}^{\text {high }}$ cells by magnetic bead. Flow cytometry dot plots depicting Ly6C expression on the population of monocytes collected by positive selection (eluate, Ly6C ${ }^{\text {high }}$ ) and the population that was not retained on the magnetic column (flow-through, Ly6 $\mathrm{C}^{\text {low }}$ ). (F) Cell lysates from each fraction were immunoblotted for FAK and Pyk2. A representative immunoblot of FAK and Pyk2 expression in lysates generated from the Ly6C ${ }^{\text {high }}$ (lane 1) and Ly6C ${ }^{\text {low }}$ (lane 2) populations is presented. ERK serves as a loading control. (G) Images display representative hematoxylin stained cytospin preparations of the $\mathrm{Ly}_{6} \mathrm{C}^{\text {high }}$ and $\mathrm{Ly} 6 \mathrm{C}^{\text {low }}$ populations. Data presented in the bar graphs are from 4 independent experiments. SEM, $*=\mathrm{P}<.05$. 

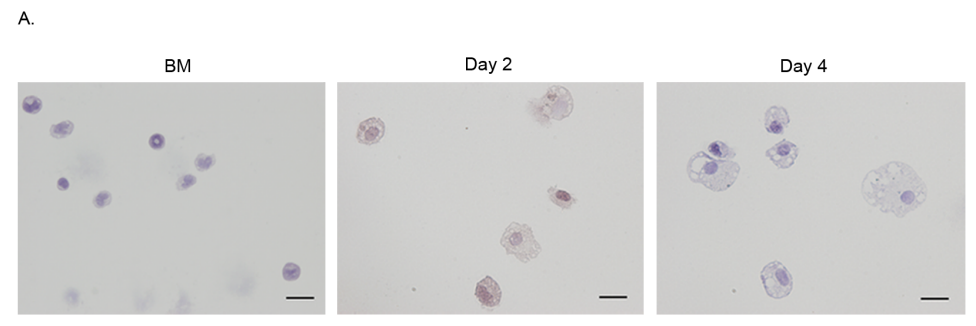

B.

c.

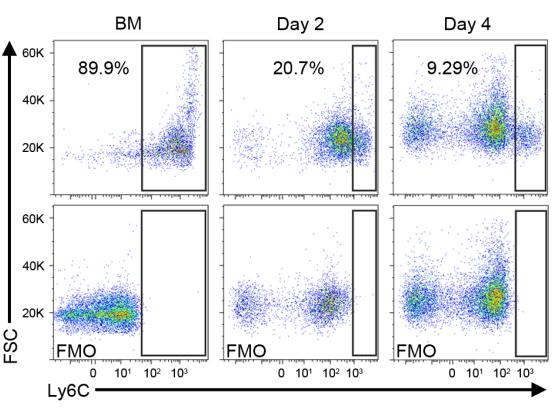

D.
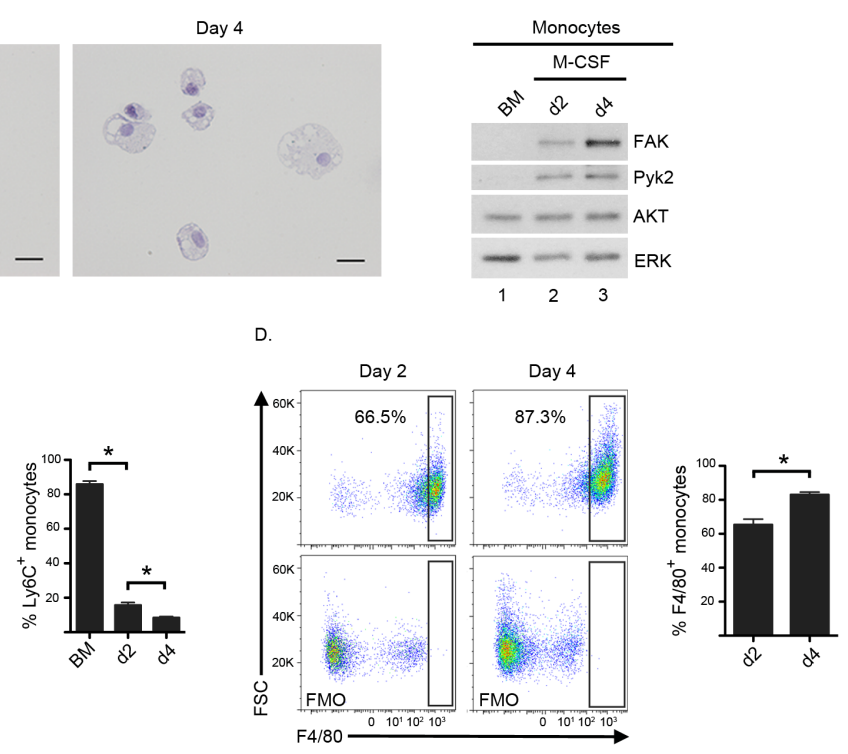

E.
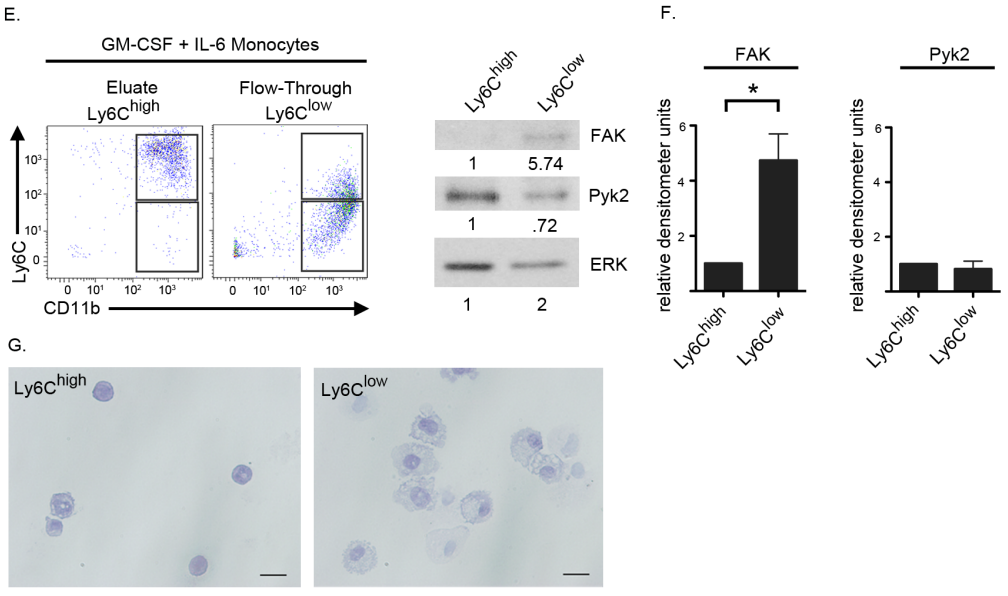
Figure 2.7 FAK expression is elevated in $\mathrm{Ly}^{\mathrm{C}} \mathrm{C}^{\mathrm{low}}$ monocytes derived from M-CSF treated BM. (A) WT BM was treated in vitro with M-CSF for three days. Adherent cells expressing Ly6G were removed by positive magnetic bead selection. The resulting flow-through, consisting of Ly6C $\mathrm{C}^{\text {high }}$ and Ly6C $\mathrm{C}^{\text {low }}$ monocytes, was separated for Ly6C $\mathrm{C}^{\text {high }}$ cells by magnetic bead. Flow cytometry dot plots depicting Ly6C expression on the population of monocytes collected by positive selection (eluate, Ly6 $\mathrm{C}^{\text {high }}$ ) and the population that was not retained on the magnetic column (flow-through, Ly6 $\mathrm{C}^{\text {low }}$ ). Images display representative hematoxylin stained cytospin preparations of the Ly6C $\mathrm{C}^{\text {high }}$ and $\mathrm{Ly} 6 \mathrm{C}^{\text {low }}$ populations. (B) Cell lysates from each fraction were immunoblotted for FAK and Pyk2. A representative immunoblot of FAK and Pyk2 expression in lysates generated from the Ly6 $\mathrm{C}^{\text {high }}$ (lane 1) and Ly6 $\mathrm{C}^{\text {low }}$ (lane 2) populations is presented. ERK serves as a loading control. Data presented in the bar graphs are from 4 independent experiments. 
A.
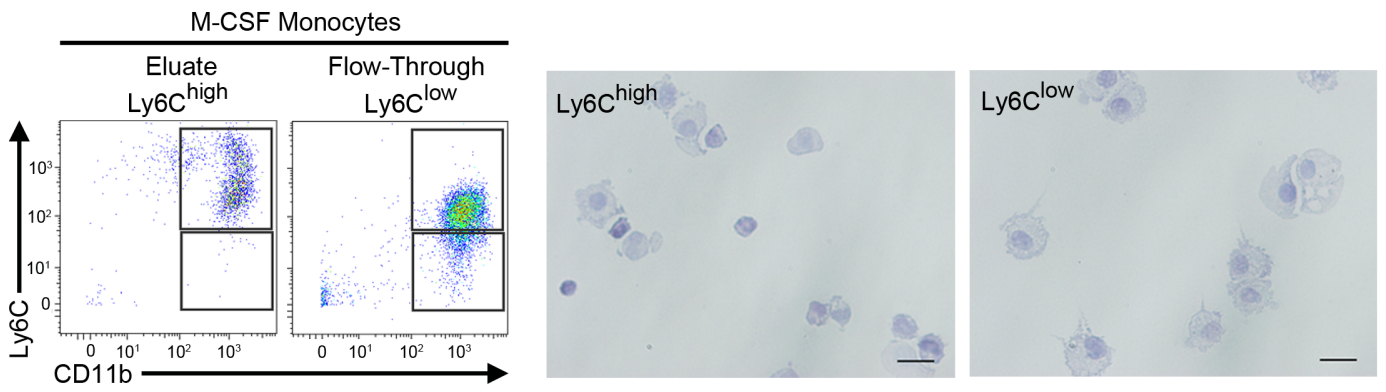

B.
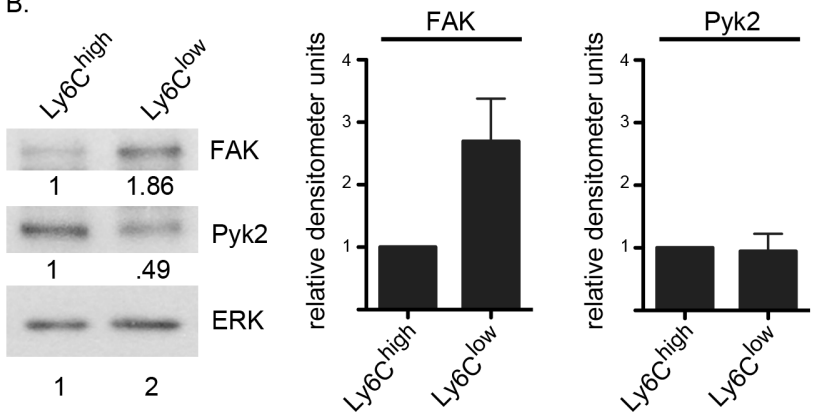
Together, these results demonstrate that, as was the case in tissues, FAK and Pyk2 protein expression is regulated during differentiation. Pyk2 is expressed in both $L y 6 C^{\text {high }}$ and Ly6C $\mathrm{C}^{\text {low }}$ cells, while FAK is expressed predominantly in Ly6C $\mathrm{C}^{\text {low }}$ monocytes. Regulation of FAK and Pyk2 protein levels may occur through several mechanisms, including at the level of transcription, through the stability of FAK and/or Pyk2 transcript, or through post-translational processes of FAK and/or Pyk2 that modify protein stability. Results from a recent study that compared gene expression profiles of $\mathrm{Gr} 1(\mathrm{Ly} 6 \mathrm{C} / \mathrm{G})^{\text {high }}$ and $\operatorname{Gr} 1(\mathrm{Ly} 6 \mathrm{C} / \mathrm{G})^{\text {low }}$ murine blood monocytes indicate that FAK and Pyk2 transcripts are present at comparable levels across the two subsets (Ingersoll et al., 2010), indicating that the increased level of FAK protein in Ly6C ${ }^{\text {low }}$ monocytes is most likely due to post-transcriptional events.

\subsubsection{FAK expression increases with the loss of Ly6C and Pyk2 expression increases with the gain of $\mathbf{F 4 / 8 0}$}

Both the loss of Ly6C and gain of F4/80 are markers of monocyte differentiation (Francke et al., 2011). We therefore wanted to characterize monocyte population dynamics in response to GMCSF + IL-6 and assess FAK and Pyk2 protein expression during differentiation as a function of Ly6C and F4/80. To determine whether Ly6 $\mathrm{C}^{\text {high }}$ monocytes from the bone marrow could differentiate into more mature cell populations upon treatment with GM-CSF + IL-6, Lin ${ }^{-}$ $\mathrm{CD} 11 \mathrm{~b}^{+} \mathrm{Ly} 6 \mathrm{C}^{\text {high }}$ monocytes were isolated by FACS from the bone marrow and cultured for two days in GM-CSF + IL-6. The starting population of cells was predominantly Ly6C ${ }^{\text {high }}$ and F4/80(Figure 2.8A), indicating that the cells were largely undifferentiated monocytes. After two days in GM-CSF + IL-6, these cells had undergone differentiation. Two populations predominated: a Ly $6 \mathrm{C}^{+} \mathrm{F} 4 / 80^{+}$population and a more differentiated $\mathrm{Ly} 6 \mathrm{C}^{-} \mathrm{F} 4 / 80^{+}$population. These data confirm that monocytes lose Ly6C and gain F4/80 during culture in GM-CSF + IL-6. 


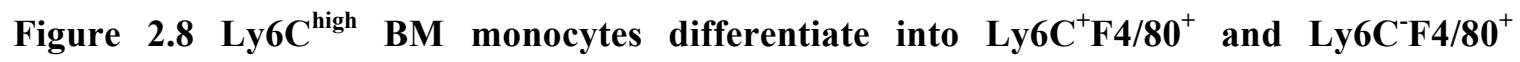
monocytes. FAK and Pyk2 expression are regulated during monocyte differentiation. (A) Lin $^{-} \mathrm{CD} 11 \mathrm{~b}^{+}$Ly6C $\mathrm{C}^{\text {high }}$ monocytes were sorted by FACS and cultured in GM-CSF (40ng/ml) + IL-6 (40ng/ml) for two days. Flow cytometry dot plots depicting Ly6C and F4/80 expression of the sorted Lin ${ }^{-} \mathrm{CD} 11 \mathrm{~b}^{+}$Ly $6 \mathrm{C}^{\text {high }} \mathrm{BM}$ monocytes at the time of plating and of adherent cells after two days treatment in GM-CSF + IL-6. (B) Proliferation of sorted Ly6C ${ }^{\text {high }}$ monocytes (left panel) and day 2 monocytes (right panel). Sorted Ly6C $\mathrm{C}^{\text {high }}$ monocytes were cultured for 3 hours in media containing BrdU prior to flow cytometry staining. The day 2 monocyte culture received a 3 hour BrdU pulse prior to cell harvest and staining. Gated populations indicate the percentage of cells from the monocyte populations identified in (A) that exhibited BrdU incorporation. FMO controls are presented for both time points. (C) WT BM was cultured in GM-CSF (40ng/ml) + IL-6 (40ng/ml) for 3 days. Adherent cells were harvested and stained with an antibody panel to distinguish $\mathrm{Lin}^{-\mathrm{CD}} 11 \mathrm{~b}^{+}$monocyte populations as a function of Ly6C and F4/80 expression. The populations were sorted by FACS and cell lysates from each population were immunoblotted for FAK and Pyk2 (Ly6C ${ }^{+} \mathrm{F} 4 / 80^{-}$, lane 1; Ly6C ${ }^{+} \mathrm{F} 4 / 80^{+}$, lane 2; Ly6C F4 $/ 80^{+}$, lane 3). AKT and ERK serve as loading controls. 
A.

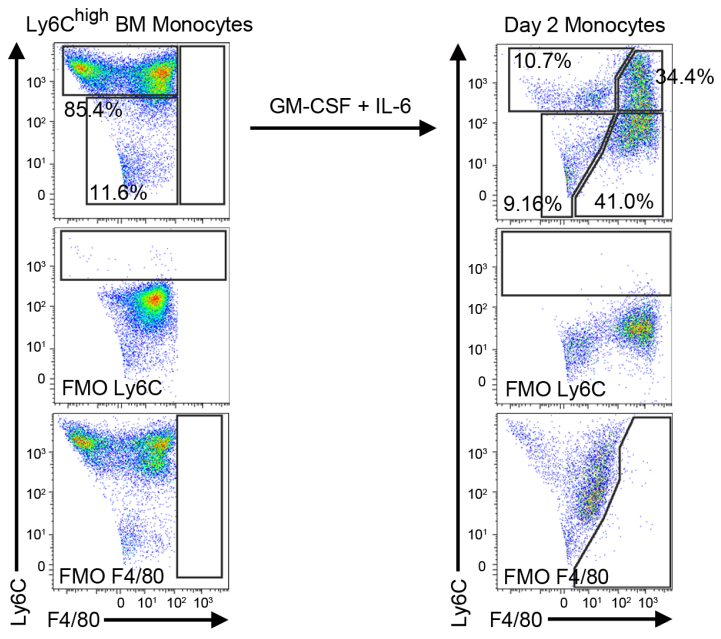

B.

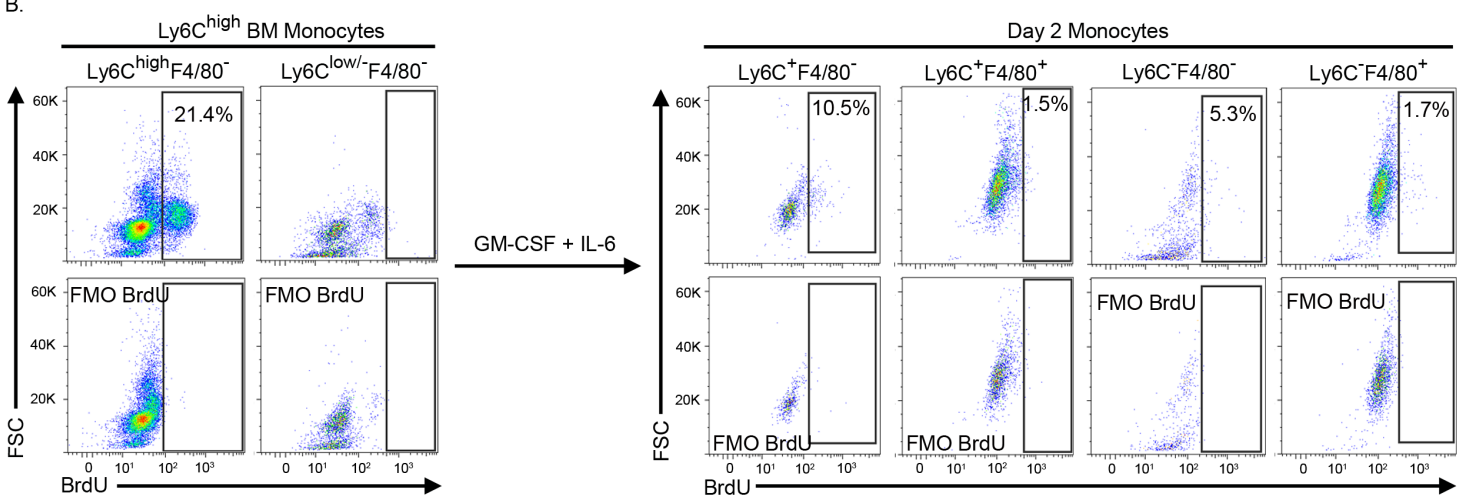


C.

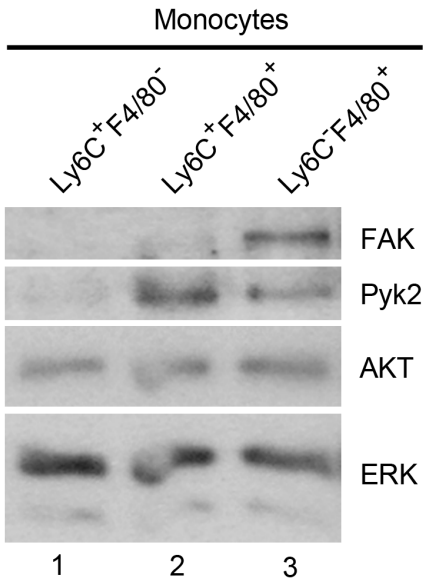


We next wanted to address if differentiation was the primary process driving the accumulation of the $\mathrm{Ly} 6 \mathrm{C}^{+} \mathrm{F} 4 / 80^{+}$and $\mathrm{Ly} 6 \mathrm{C}^{-} \mathrm{F} 4 / 80^{+}$populations, or if proliferation contributed to the accumulation of these cells. Analysis of BrdU incorporation in the enriched $\mathrm{Ly} 6 \mathrm{C}^{\text {high }} \mathrm{F} 4 / 80^{-}$population from the $\mathrm{BM}$ showed that $21 \%$ of the cells were proliferating (Figure $2.8 \mathrm{~B}$ ), consistent with what has been established as the self-renewing capacity of Ly6C $C^{\text {high }}$ BM monocytes (Hettinger et al., 2013). GM-CSF+IL-6 treatment resulted in a significant reduction in BrdU incorporation in these cells, suggesting that they had in large part lost their ability to proliferate. These results argue that the $\mathrm{Ly}_{6 \mathrm{C}} \mathrm{F}^{\mathrm{F}} 4 / 80^{+}$and $\mathrm{Ly}^{-} \mathrm{C}^{-} \mathrm{F} 4 / 80^{+}$populations present 2 days after GM-CSF+IL6 treatment were likely generated through a process of differentiation and not from proliferating cells within each population.

To determine the level of FAK and Pyk2 expression in these populations, BM was cultured for three days in GM-CSF+IL-6, after which $\operatorname{Lin}^{-} \mathrm{CD} 11 \mathrm{~b}^{+}$cells were sorted by FACS. FAK expression was undetectable in $\mathrm{Ly} 6 \mathrm{C}^{+} \mathrm{F} 4 / 80^{-}$monocytes, slightly elevated in $\mathrm{Ly} 6 \mathrm{C}^{+} \mathrm{F} 4 / 80^{+}$ monocytes, and expressed at high levels in the population that lost Ly6C (Figure 2.8C). In contrast, Pyk2 expression became significantly elevated coincident with the Ly6 $\mathrm{C}^{+}$monocytes gaining F4/80 surface expression. As was the case in tissues, these data demonstrate that Pyk2 is expressed earlier than FAK during monocyte differentiation.

\subsubsection{FAK and Pyk2 regulate monocyte differentiation and/or survival in response to GM- CSF + IL-6}

As was presented in Figure 2, the BM, blood, and spleen of Pyk2\% mice contained a reduced proportion of $\mathrm{Ly}_{6 \mathrm{C}} \mathrm{C}^{+} \mathrm{F} 4 / 80^{+}$monocytes. In vitro, $\mathrm{FAK}$ was expressed predominantly in the most differentiated monocytes after surface expression of Ly6C was lost $\left(\mathrm{Ly}^{-} \mathrm{C}^{-} \mathrm{F} 4 / 80^{+}\right)$, whereas Pyk2 expression was present in $\mathrm{Ly} 6 \mathrm{C}^{+}$cells coincident with the appearance of surface expression of F4/80. However, while there was a clear difference in the temporal regulation of these molecules, 
Figure 2.9 FAK and Pyk2 regulate monocyte differentiation. BM from WT, FAK ${ }^{\Delta \text { myeloid }}$, and Pyk2\% mice was cultured in the presence of GM-CSF (40ng/ml) + IL-6 (40ng/ml) (A) and (B) or M-CSF (C) and (D) for 2 and 4 days. (A) Adherent cells at each time point in GM-CSF + IL-6 cultures were stained by an antibody panel to distinguish $\mathrm{Ly}_{6 \mathrm{C}^{+}} \mathrm{F} 4 / 80^{-}, \mathrm{Ly}_{6 \mathrm{C}^{+}} \mathrm{F} 4 / 80^{+}$, and $\mathrm{Ly} 6 \mathrm{C}^{-}$ $\mathrm{F} 4 / 80^{+}$monocytes. Bar graphs display the percentage of cells present in the gated populations on the flow cytometry dot plots for each time point. Statistical significance was determined by comparing the percentage of Ly6 $\mathrm{C}^{+} \mathrm{F} 4 / 80^{-}$monocytes to Ly $6 \mathrm{C}^{+} \mathrm{F} 4 / 80^{+}$monocytes at each time point and the percentage of $\mathrm{LyC}^{-} \mathrm{F} 4 / 80^{+}$monocytes at day 2 compared to day 4 . (B) The percentage of cells within each gate from WT cultures was compared to the complementary gate from $\mathrm{FAK}^{\Delta \text { myeloid }}$ and Pyk2\% cultures. (C) Flow cytometry dot plots of day 2 and day 4 monocytes in M-CSF. (D) The percentage of cells within each gate from WT cultures was compared to the complementary gate from $\mathrm{FAK}^{\Delta \text { myeloid }}$ and $\mathrm{Pyk}^{\%} \%$ cultures. Data are representative of $7 \mathrm{WT}, 7$ $\mathrm{FAK}^{\Delta \text { myeloid }}$, and $4 \mathrm{Pyk}^{-\%}$ replicates for each condition. SEM, $*=\mathrm{P}<.05$. 

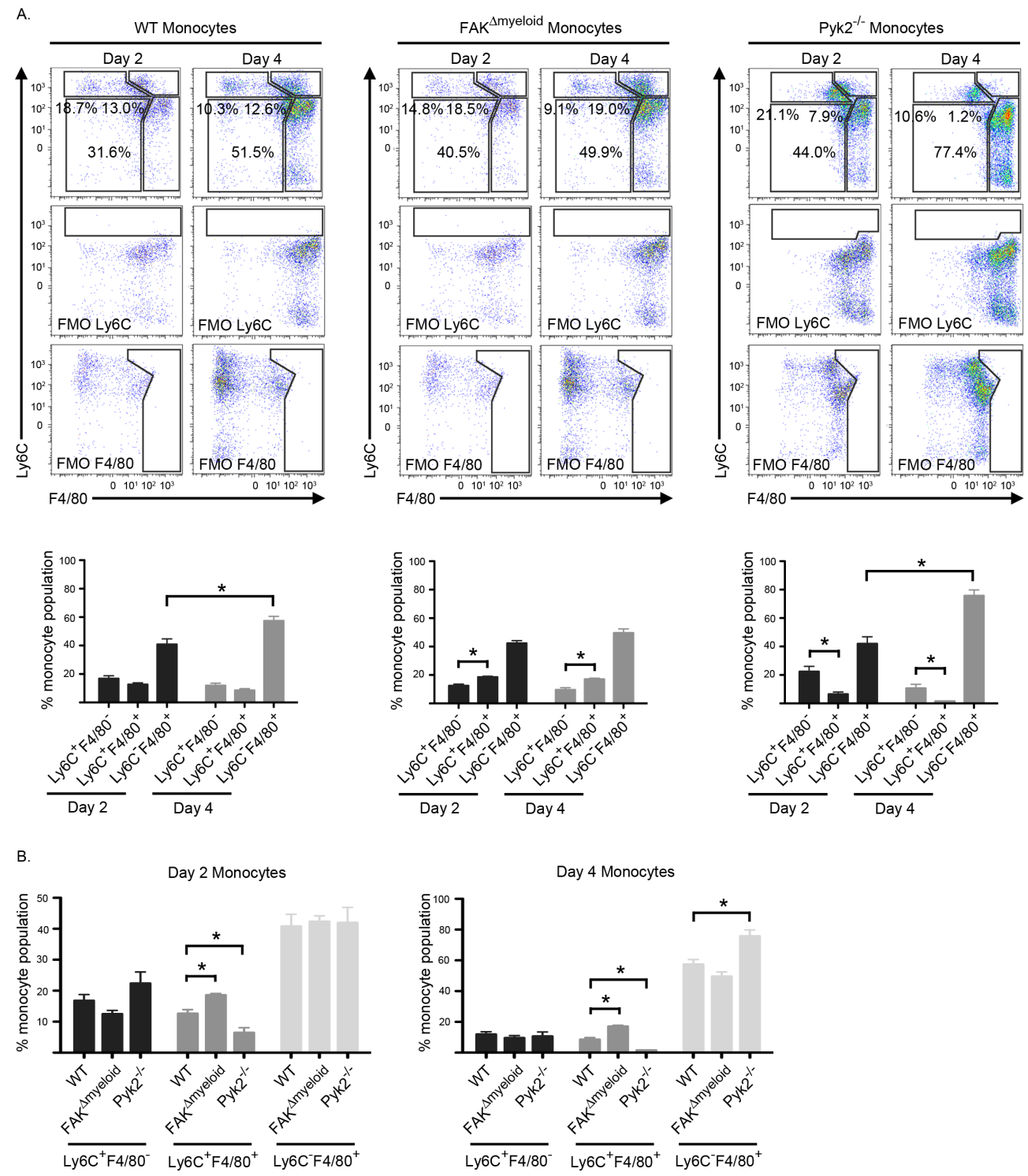
C.

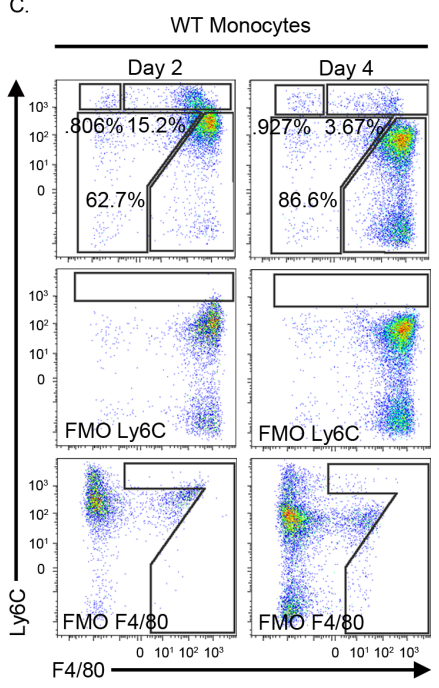

D.

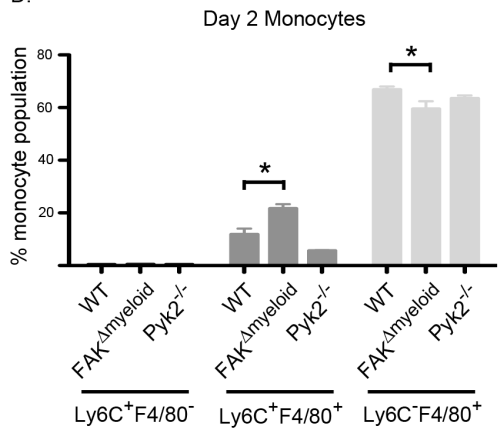

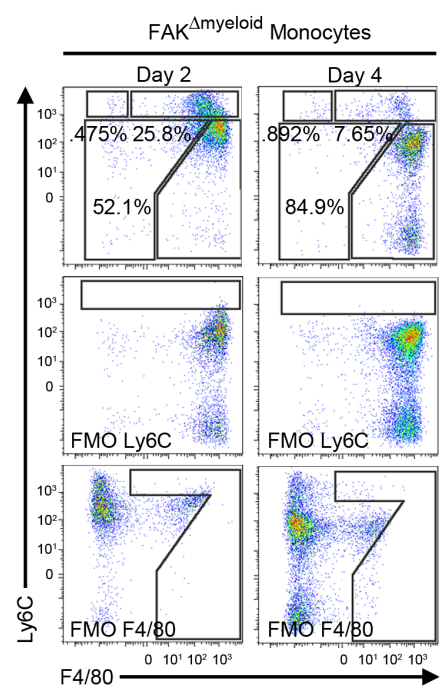

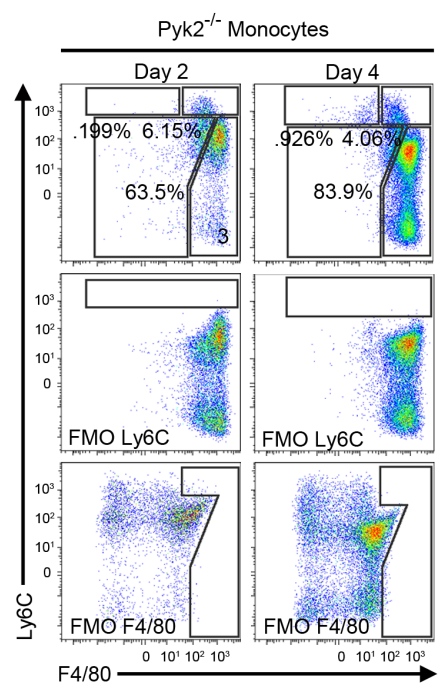

Day 4 Monocytes

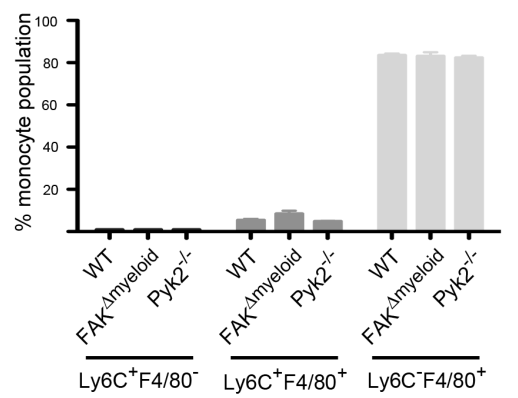


it is not clear from these data whether upregulation of FAK and Pyk2 expression is a cause or consequence of monocyte differentiation, and/or if FAK and Pyk2 regulate monocyte survival. To address this, we established an in vitro system using Ly6C and F4/80 as markers to track monocyte differentiation. BM from WT, $\mathrm{FAK}^{\Delta \text { myeloid }}$, and $\mathrm{Pyk}^{-\digamma}$ mice was cultured for two and four days in GM-CSF+IL-6. In the WT Lin $^{-} \mathrm{CD} 11 \mathrm{~b}^{+}$monocyte populations, Ly6C ${ }^{-} \mathrm{F} 4 / 80^{+}$cells were most highly represented (Figure 2.9A). Moreover, the percentage of these cells increased from Day 2 to Day 4, consistent with the generation of more highly differentiated cells over time. This population was also enriched in cultures from FAK-deficient mice, but in contrast to WT cells, it did not increase over time. This was coincident with a small but statistically significant increase in the percentage of the less differentiated $\mathrm{Ly} 6 \mathrm{C}^{+} \mathrm{F} 4 / 80^{+}$cells at both time points relative to WT cells (Figure 2.9B). The reverse was true of Pyk2\% cells. The most differentiated Ly6C ${ }^{-}$ F4/80 $0^{+}$cells exhibited a more significant increase from Day 2 to Day 4 than did WT cells (Figure 2.9A), while the less mature $\mathrm{Ly} 6 \mathrm{C}^{+} \mathrm{F} 4 / 80^{+}$cells were significantly depleted compared to WT cells (Figure 2.9B). Together, these results suggest that FAK functions to promote differentiation of Ly6 $\mathrm{C}^{+} \mathrm{F} 4 / 80^{+}$monocytes and/or survival of Ly6C-F4/80+ macrophages. Conversely, Pyk2 induces differentiation of $\mathrm{Ly} \mathrm{C}^{+} \mathrm{F} 4 / 80^{-}$monocyte, blocks the differentiation of Ly6 $\mathrm{C}^{+} \mathrm{F} 4 / 80^{+}$monocytes, and/or regulates survival of Ly6C+F4/80+ monocytes. The fact that there were no significant differences between the $\mathrm{Ly}_{6 \mathrm{C}^{+}} \mathrm{F} 4 / 80^{-}$populations generated from any of the mice, combined with the fact that neither Pyk2 nor FAK are expressed in these cells, suggests that FAK and Pyk2 are not likely to be involved in the differentiation or survival of the Ly6 $\mathrm{C}^{+} \mathrm{F} 4 / 80^{-}$monocyte subset. Moreover, BrdU incorporation in day $2 \mathrm{Ly} 6 \mathrm{C}^{+}$monocytes from WT, $\mathrm{FAK}^{\Delta \text { myeloid }}$, and $\mathrm{Pyk} 2 \%$ mice was similar, suggesting that proliferation was not responsible for the differences we observed in the proportion of monocyte populations across the three genotypes (Figure 2.10). 
Figure 2.10 BrdU incorporation is equivalent in $\mathrm{Ly}^{+} \mathrm{C}^{+}$monocytes derived from GM-CSF + IL-6 treated BM. Whole bone marrow was cultured in GM-CSF [40ng/ml]+IL-6 [40ng/ml] for two days, at which time the cultures were pulsed with BrdU for 3 hours. The adherent cells were harvested and stained for flow cytometry. BrdU incorporation in the $\operatorname{Lin}^{-} \mathrm{CD} 11 \mathrm{~b}^{+} \mathrm{Ly} 6 \mathrm{C}^{+}$ monocytes in indicated by gate. 


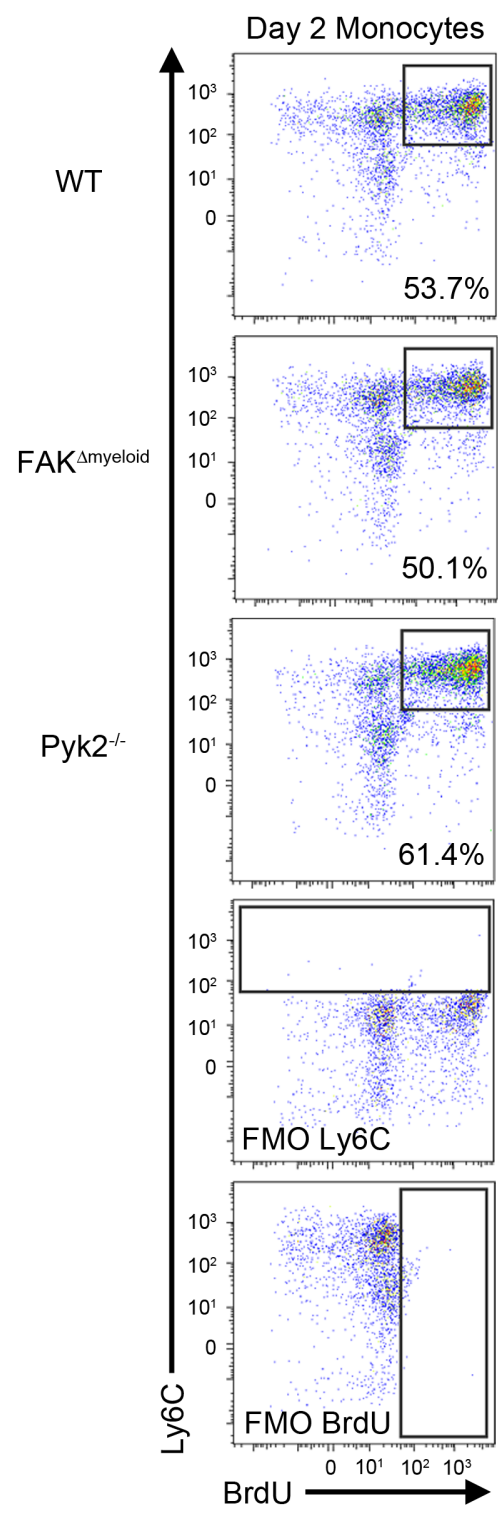


To determine if FAK and Pyk2 regulation of monocyte differentiation and/or survival was specific to GM-CSF + IL-6, we repeated the experiment above using M-CSF as a differentiationinducing factor. It has been demonstrated that sorted BM Ly6 $\mathrm{C}^{\text {high }}$ monocytes cultured in M-CSF differentiate predominantly into $\mathrm{LyC}^{+} \mathrm{F} 4 / 80^{+}$and $\mathrm{Ly} 6 \mathrm{C}^{-} \mathrm{F} 4 / 80^{+}$populations (Mazzon et al., 2012). Using this condition, over $60 \%$ of the WT monocytes were Ly $6 \mathrm{C}^{-} \mathrm{F} 4 / 80^{+}$by day 2 , and this increased to over $80 \%$ by day 4 (Figure $2.9 \mathrm{C}$ and $2.9 \mathrm{D}$ ). The least differentiated $\mathrm{Ly}_{6 \mathrm{C}^{+} \mathrm{F} 4 / 80^{-}}$ population was negligible at both time points, suggesting that monocyte differentiation had progressed more rapidly in M-CSF than in GM-CSF+IL-6 (compare panels 2.9A with 2.9C). As was the case for GM-CSF+IL-6, however, the $\mathrm{Ly} 6 \mathrm{C}^{+} \mathrm{F} 4 / 80^{+}$population was over-represented in the FAK-depleted cultures. There was a coincident modest decrease in the percentage of Ly6 $\mathrm{C}^{-}$ $\mathrm{F} 4 / 80^{+}$macrophages, indicating that $\mathrm{FAK}$ drives differentiation in response to M-CSF or enhances survival of $\mathrm{Ly}^{-} \mathrm{C}^{-} \mathrm{F} 4 / 80^{+}$macrophages. These differences were not seen at day 4 , perhaps because the number of cells in this population was so low.

\subsubsection{FAK regulates $\mathrm{Ly}^{\mathrm{low}} \mathrm{C}^{\mathrm{low}}$ monocyte migration}

FAK is expressed predominantly in $\mathrm{Ly} 6 \mathrm{C}^{-}$monocytes compared to $\mathrm{LyC}^{+}$monocytes. Since FAK was shown to be important for migration of bone marrow derived macrophages toward M-CSF and other factors, we next wanted to determine if $\mathrm{Ly} 6 \mathrm{C}^{\text {high }}$ and $\mathrm{Ly} 6 \mathrm{C}^{\mathrm{low}}$ monocytes derived from $\mathrm{FAK}^{\Delta \mathrm{myeloid}}$ mice migrated as efficiently as analogous populations derived from WT mice. BM from WT and FAK ${ }^{\Delta \text { myeloid }}$ mice was cultured in GM-CSF + IL-6 and immunomagnetic column separation was employed to isolate Ly6 $\mathrm{C}^{\text {high }}$ and Ly6 $\mathrm{C}^{\text {low }}$ monocytes. An equivalent number of cells from each population was plated in the upper chamber of transwell inserts and the cells were allowed to migrate toward M-CSF for 16 hours. WT Ly6 $\mathrm{C}^{\text {low }}$ monocytes displayed enhanced migration to M-CSF relative to WT Ly6 $\mathrm{C}^{\text {high }}$ monocytes (Figure 2.11A). This was of note given the comparatively elevated levels of FAK in this population of cells. We observed no difference in the migratory capability of WT and FAK ${ }^{\Delta \text { myeloid }}$ Ly6 $\mathrm{C}^{\text {high }}$ monocytes. However, the absence of 
Figure 2.11 FAK regulates $\mathrm{Ly} \mathrm{C}^{\text {low }}$ monocyte migration. BM from $\mathrm{WT}$ and $\mathrm{FAK}{ }^{\Delta \text { myeloid }}$ mice was cultured in GM-CSF + IL-6 and immunomagnetic column separation was employed to isolate Ly6 $\mathrm{C}^{\text {high }}$ and Ly6 $\mathrm{C}^{\text {low }}$ monocytes. (A) Equivalent numbers of Ly6C $\mathrm{C}^{\text {high }}$ and $\mathrm{Ly} 6 \mathrm{C}^{\text {low }}$ monocytes were plated in the upper chamber of transwell inserts and allowed to migrate to MCSF for 16 hours. Migration of cells in sister transwell inserts toward serum free media established the background, or random, migration. Data are representative of 3 independent experiments. SEM, * $=\mathrm{P}<.05$. (B) Ly6 $\mathrm{C}^{\text {low }}$ monocytes were analyzed by flow cytometry for surface expression of M-CSFR. Migration experiments conducted by Ryan Llewellyn; M-CSFR flow cytometry completed by MFG. 


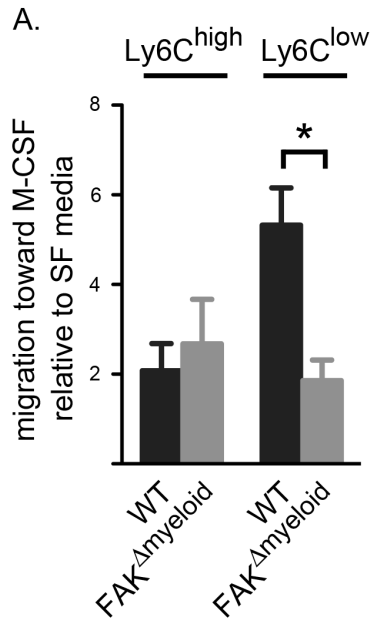

B.

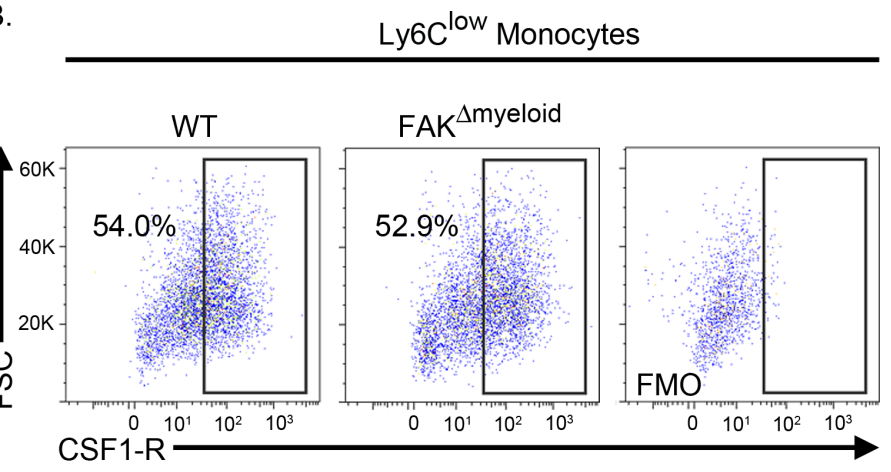


FAK in Ly6 $\mathrm{C}^{\text {low }}$ monocytes resulted in a significant reduction in the number of cells that migrated toward M-CSF compared to WT Ly6C ${ }^{\text {low }}$ cultures. Differential expression of the M-CSF receptor could not account for differences in migration of the WT and $\mathrm{FAK}^{\Delta \text { myeloid }} \mathrm{Ly} 6 \mathrm{C}^{\text {low }}$ cells, as the same percentage of cells expressed the receptor, and the receptor was expressed at equivalent levels in both populations (MFI CSF1-R; WT 97.2, FAK (myeloid $_{117)}$ (Figure 2.11B). These results demonstrated that FAK regulates Ly $6 \mathrm{C}^{\text {low }}$ but not Ly $6 \mathrm{C}^{\text {high }}$ monocyte migration toward MCSF.

\subsection{Discussion}

Cells of the mononuclear phagocyte system play prominent roles in steady state tissue homeostasis and disease prevention. The regulated expression of signaling molecules that orchestrate effector cell trafficking and activity must be carefully controlled to establish a balance between homeostasis and inflammation. Numerous examples illustrate how alterations to this process shift a system designed to promote development and protect self into one that potentiates disease (Murray and Wynn, 2011).

We demonstrate in this report that FAK and Pyk2 expression in lineage committed CD11b ${ }^{+}$ monocytes is regulated during monocyte differentiation. Our data indicate that protein expression of Pyk2 is initiated prior to FAK as monocytes differentiate, at the stage when surface expression of F4/80 is first evident (Figure 2.12A). FAK is not expressed until a later stage, after surface expression of Ly6C is lost. The parallels we observed in vivo and in vitro suggest that the regulated expression of FAK and Pyk2 in monocytes is fundamental, and argue that functional properties attributed to monocyte populations distinguished by Ly6C and F4/80 expression are modulated by FAK and Pyk2. 
Monocyte populations defined by the surface expression of Ly6C are uniquely regulated and functionally distinct. In the $\mathrm{BM}, \mathrm{Ly} 6 \mathrm{C}^{+}$monocytes retain proliferative potential and exist as precursors to $\mathrm{Ly}^{-} \mathrm{C}^{-} \mathrm{BM}$ monocytes as well as to $\mathrm{Ly}_{6} \mathrm{C}^{+}$monocytes in the blood (Yona et al., 2013). In the blood, cells expressing high levels of Ly6C are elevated in number under inflammatory conditions and display a dependence on CCL2 for migration (Kamei and Carman 2010). When these cells lose Ly6C, they gain CX3CR1 expression, utilize LFA-1 for migration, become longer-lived, and are charged with filling an entirely new functional role of blood vessel patrol (Auffray et al., 2007). In the spleen, monocytes generated through local myelopoiesis mobilize during inflammatory conditions in vivo in a similar manner to blood monocytes and differentiate to both macrophages and dendritic cells when cultured ex vivo (Swirski et al., 2009).

We show that Pyk2 is required to maintain the proportion of BM, blood, and splenic monocytes that express both Ly6C and F4/80 (Ly6C $\mathrm{F}^{+} 4 / 80^{+}$) (see Figure 2.4). Evidence indicated that the reduced $\mathrm{Ly} 6 \mathrm{C}^{+} \mathrm{F} 4 / 80^{+}$tissue populations in $\mathrm{Pyk} 2^{-/-}$mice are not due to proliferation or trafficking defects. Proliferation is limited to the $\mathrm{BM}$ and splenic $\mathrm{CD} 11 \mathrm{~b}{ }^{-} \mathrm{Ly} 6 \mathrm{C}^{-}$monocyte-macrophage dendritic cell progenitors (MDP) and $\mathrm{CD} 11 \mathrm{~b}^{-} \mathrm{Ly} 6 \mathrm{C}^{\text {high }}$ committed monocyte progenitors (cMoP), and to a much lesser degree $\mathrm{CD} 11 \mathrm{~b}^{+}$Ly6 $\mathrm{C}^{+}$monocytes in the bone marrow (Hettinger et al., 2013). Additionally, MDP, cMoP, and proliferating Ly6C ${ }^{+}$monocytes are not present in the blood. Our data showed that $\mathrm{Ly}_{6 \mathrm{C}}^{+}$monocyte proliferation in the bone marrow was equivalent in wildtype, $\mathrm{FAK}^{\Delta \text { myeloid }}$, and Pyk2 $2^{-/-}$mice. A second possible explanation for the reduced proportion of Ly6C $\mathrm{C}^{+} 4 / 80^{+}$blood and splenic monocytes in Pyk $2^{-/}$mice could be compromised trafficking due to the absence of Pyk2. This seemed unlikely for several reasons. First, the Ly $6 \mathrm{C}^{+} \mathrm{F} 4 / 80^{+}$ population was reduced in all three tissues, suggesting a broader phenomenon than impaired trafficking. Second, there was no reduction in the percentage of blood Ly $6 \mathrm{C}^{+} \mathrm{F} 4 / 80^{-}$monocytes, which might also have been expected if trafficking was impaired. Third, this analysis was conducted at steady state and not under conditions favoring a high rate of $\mathrm{Ly}_{6 \mathrm{C}} \mathrm{C}^{+}$monocyte egress 
from the BM. Based on these factors, the reduced proportion of $\mathrm{BM}$, blood, and splenic Ly6 $\mathrm{C}^{+} \mathrm{F} 4 / 80^{+}$monocytes in the Pyk2 $2^{-/}$mice was most likely the result of Pyk2 regulation of monocyte differentiation and/or survival, and not proliferation or trafficking. However, Pyk2 does become significantly upregulated in this population of cells, indicating that it does in fact play a functional or regulatory role in this population. Using an in vitro system of differentiation, we observed a decrease in the $\mathrm{Ly} 6 \mathrm{C}^{+} \mathrm{F} 4 / 80^{+}$population and a concomitant increase in the more differentiated Ly6C F4 $4 / 80^{+}$monocytes in the absence of Pyk2.

Our combined data indicate that FAK and Pyk2 may function in two capacities to regulate the accumulation of monocyte populations. One potential mechanism is through the modulation of monocyte differentiation, in which case Pyk2 would act as a brake on differentiation to restrict cells from becoming fully differentiated Ly6 $\mathrm{C}^{-} \mathrm{F} 4 / 80^{+}$macrophages (Figure $2.12 \mathrm{~A}$, bottom). In contrast to Pyk2, FAK expression becomes upregulated in the most highly differentiated Ly6C ${ }^{-}$ $\mathrm{F} 4 / 80^{+}$population. Deletion of $\mathrm{FAK}$ resulted in an accumulation of $\mathrm{Ly} 6 \mathrm{C}^{+} \mathrm{F} 4 / 80^{+}$monocytes in GM-CSF + IL-6 and M-CSF conditions, and a reduction in Ly6CF4 $/ 80^{+}$cells with M-CSF treatment. This suggests that FAK may function as an accelerator in the later stages of monocyte differentiation, pushing cells to become fully differentiated macrophages.

The proposed functions of Pyk2 as a "brake" and FAK as an "accelerator" of monocyte differentiation has important ramifications in the BM and blood (Figure 2.12B). In both tissues, maintaining a robust precursor population is important for being able to quickly adapt to the needs of peripheral tissues. The temporal regulation of first Pyk2 and then FAK expression allows for the brake to precede the accelerator, which could help maintain an appropriate balance between precursor monocyte and effector macrophage. In $\mathrm{CD} 34^{+}$progenitor cells, it was shown that Pyk2 mediates signaling through integrins to inhibit differentiation (Dylla et al., 2004). Our data indicate that a similar process may occur in the more differentiated BM and blood monocytes. 
Figure 2.12 Potential mechanisms of FAK and Pyk2 regulation of monocyte differentiation, survival, and/or function. (A) Pyk2 is expressed earlier than FAK during monocyte differentiation and functions as a brake in the differentiation of $\mathrm{Ly} 6 \mathrm{C}^{+} \mathrm{F} 4 / 80^{+}$monocytes or regulates survival. Pyk2 expression increases as monocytes increase surface expression of F4/80. FAK is expressed as monocytes lose Ly6C. FAK functions to drive the differentiation of $\mathrm{LyC}^{+} \mathrm{F} 4 / 80^{+}$monocytes or regulates survival of Ly6C $\mathrm{F}^{-} 4 / 80^{+}$macrophages. FAK and Pyk2 play opposing roles at this stage of differentiation. (B) FAK is expressed predominantly in Ly6C ${ }^{\text {low }}$ monocytes, whereas Pyk2 is expressed in both Ly6C $\mathrm{C}^{\text {high }}$ and Ly6C $\mathrm{C}^{\text {low }}$ monocytes. Pyk2 may regulate the differentiation of $\mathrm{Ly}^{+} \mathrm{C}^{+}$monocytes (steps 1 and 3), the trafficking of $\mathrm{Ly} 6 \mathrm{C}^{+}$ monocytes (steps 2 and 6), and/or the trafficking and function of Ly6 $\mathrm{C}^{-}$monocytes (steps 4,5, and 7). We would hypothesize that FAK is most likely to play a predominant role in the trafficking (steps 4 and 7) and migration (step 5) of $\mathrm{Ly}^{-} \mathrm{C}^{-}$monocytes. Both FAK and Pyk2 may regulate Ly6C $\mathrm{F}^{-} 4 / 80^{+}$macrophage function in peripheral tissues. 
A.

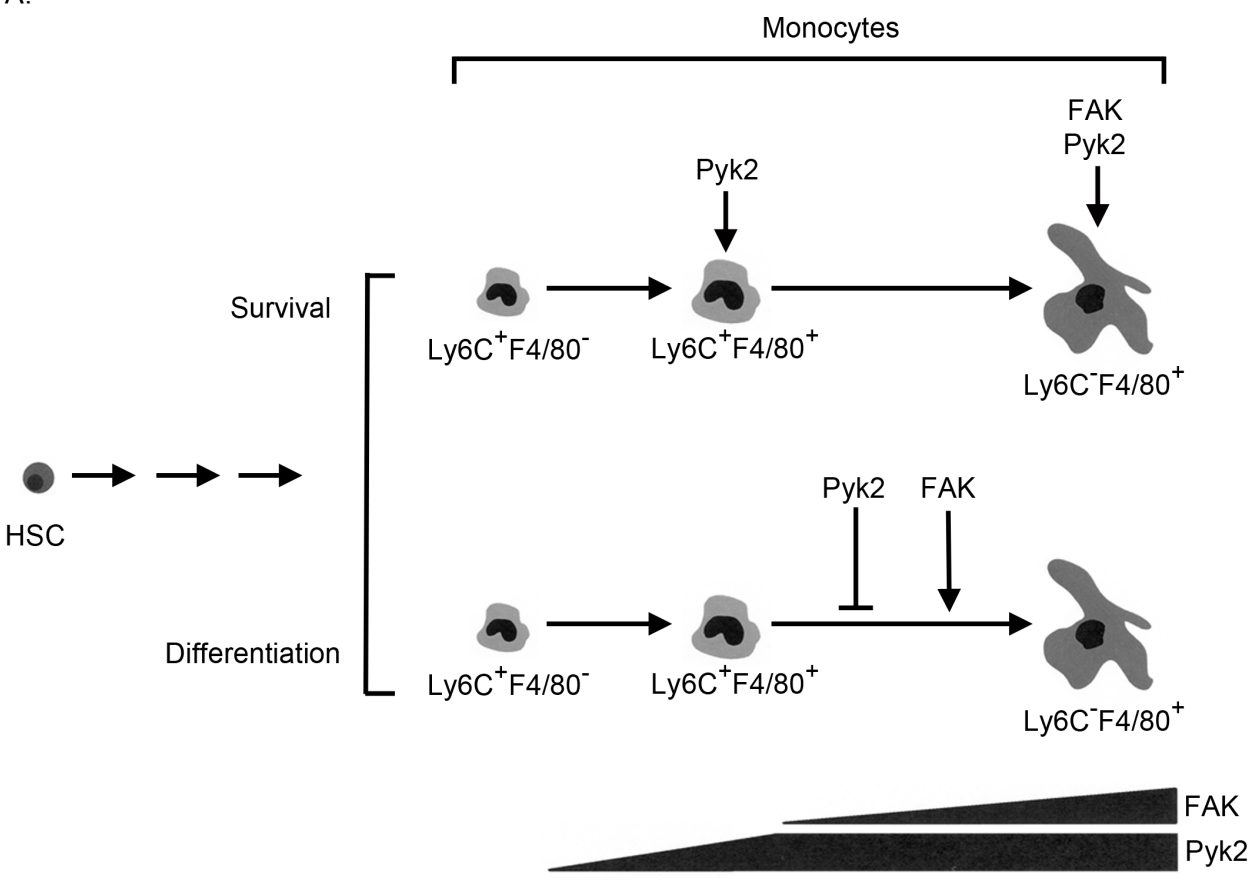

B.

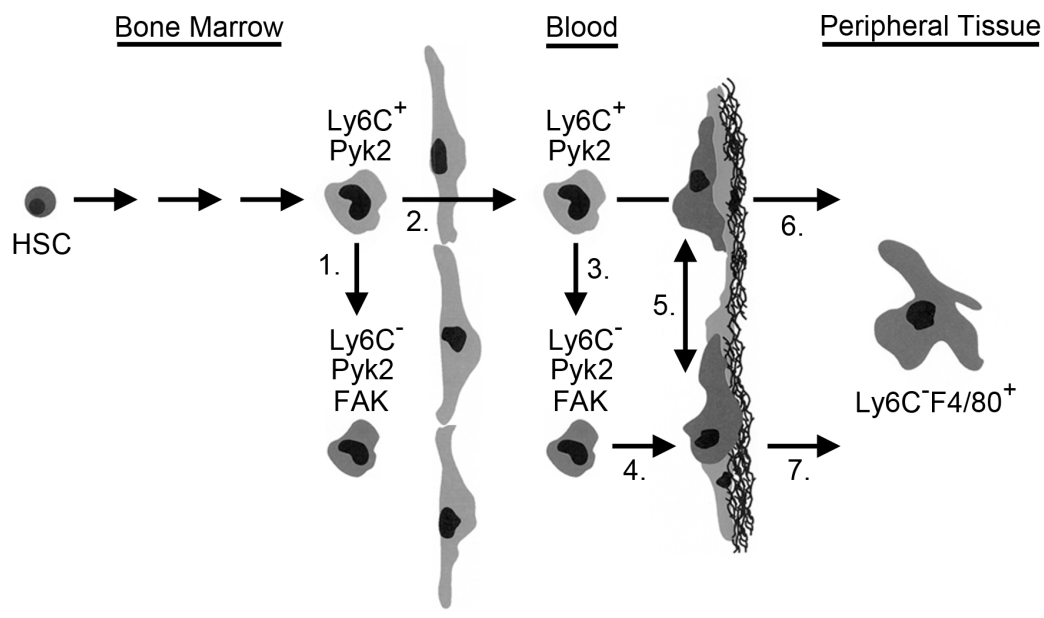


An alternative explanation for our results is that FAK and Pyk2 are involved in regulating monocyte survival (Figure 2.12A, top). Evidence exists in other cell types that both molecules regulate this process (Lim et al., 2008; Lim et al., 2010). Our finding that the ablation of Pyk2 resulted in a reduced proportion of $\mathrm{Ly} 6 \mathrm{C}^{+} \mathrm{F} 4 / 80^{+}$monocytes in vivo and in response to GM-CSF + IL-6 strongly suggests that Pyk2 may function in this capacity. FAK would be unable to compensate for the survival defect associated with the loss of Pyk2 given that it is not expressed in these cells. Our data indicate, however, that FAK may regulate survival of Ly6C $\mathrm{F}^{-} / 80^{+}$ macrophages, as we observed a reduced proportion of this population under M-CSF conditions when FAK was deleted. Future studies utilizing $\mathrm{FAK}^{\Delta \text { myeloid }}$ and $\mathrm{Pyk}^{-/-}$mice that incorporate analysis of pro-apoptotic proteins in the monocyte populations discussed above will help to elucidate the contributions of FAK and Pyk2 in regulating monocyte differentiation and survival.

There are also functional implications to consider beyond the regulation of monocyte differentiation and survival. Our data indicate that FAK regulation of mononuclear phagocyte activity is limited to $\mathrm{Ly} 6 \mathrm{C}^{-}$monocytes and macrophages. Ly6 $\mathrm{C}^{-}$monocytes comprise roughly $50 \%$ of circulating monocytes and exhibit a distinctive phenotype. They have been shown to patrol and scavenge particles on the endothelium, as well as extravasate rapidly in response to inflammatory signals. A high migratory capacity is a hallmark of these cells. We have previously shown that FAK regulates migration of bone marrow-derived macrophages and monocyte recruitment during peritoneal inflammation (Owen et al., 2007). We hypothesize that FAK expression in $\mathrm{Ly}_{6 \mathrm{C}}$ blood monocytes regulates the initial attachment, patrolling nature, and/or extravasation of these cells. One mechanism of regulation could be through the interaction of CX3CR1, which is highly expressed on Ly6C monocytes, fractalkine (FKN), the ligand for CX3CR1 expressed on endothelial cells, and $\alpha 4 \beta 1$ integrin, which signals through FAK. Disruption of FKN binding to $\alpha 4 \beta 1$ leads to the inhibition of CX3CR1-FKN- $\alpha 4 \beta 1$ ternary complex formation and reduced integrin activation (Fujita et al., 2012). Disruption of either 
CX3CR1-mediated signaling or $\alpha 4 \beta 1$ signaling through the loss of FAK could have implications in the transition of initial tethering events, mediated by FKN and CX3CR1, to monocyte rolling and firmer adhesion involving $\alpha 4 \beta 1$.

Once in tissues, FAK may regulate $\mathrm{Ly}^{6} \mathrm{C}^{-}$monocyte differentiation, survival, and/or function. We have shown previously that macrophages deficient in FAK display impaired migration to both chemokines and growth factors (M-CSF, MCP-1, SDF-1 $\alpha$ ), which suggests that FAK is involved in macrophage response to these stimuli in the tissue milieu (Owen et al., 2007) A potential mechanism of regulation is through altered IL-6 signaling. In addition to its role as a monocyte chemoattractant, IL-6 can promote macrophage differentiation through increased M-CSF receptor expression and enhanced autocrine M-CSF signaling (Clahsen and Schaper, 2008; Chomarat et al., 2000). The upregulation of FAK as monocytes differentiate may be coordinated with either or both of these responses in the tissue microenvironment.

While we have shown that Pyk2 maintains the Ly6C $\mathrm{C}^{+} \mathrm{F} / 80^{+}$monocyte population in steady state and in response to GM-CSF + IL-6, and that FAK modulates their accumulation during M-CSF treatment, we do not know if either molecule regulates the functional activity of $\mathrm{Ly} 6 \mathrm{C}^{+} \mathrm{F} 4 / 80^{+}$ monocytes. Defining this population by Ly6C and F4/80 alone does not address the functional status of these cells, as these markers have been used to denote both inflammatory monocytes and tumor-associated macrophages derived from myeloid derived suppressor cells (Movahedi et al., 2010; Corzo et al., 2010; Van Ginderachter et al., 2006). Animal models that promote inflammation or tumor growth, and thus harbor large proportions of Ly $6 \mathrm{C}^{+} \mathrm{F} 4 / 80^{+}$monocytes, may help to determine the functional significance of the regulated expression of FAK and Pyk2 in monocytes. 


\section{Chapter 3}

The regulation of murine breast tumor outgrowth and mononuclear phagocyte function by focal adhesion kinase (FAK)

\subsection{Introduction}

Tumor associated mononuclear phagocyte plasticity, effector function, and accumulation in the tumor microenvironment are governed by the ability of these cells to interpret extracellular stimuli. Focal adhesion kinase (FAK) is an established mediator of signaling cascades involving factors commonly associated with tumors, including growth factors, chemokines, and extracellular matrix. FAK has also been implicated in the coordinated crosstalk between pathways initiated through integrin engagement and growth factor receptor signaling, and it has been demonstrated repeatedly in several cell types that disruption of FAK activity affects cell migratory behavior (Sieg et al., 2000; Owen et al., 2007). We have also found that FAK expression is elevated in more differentiated monocytes and that it promotes differentiation in response to M-CSF (Chapter 2). Additionally, it has been shown that FAK mediates signaling through M-CSF in human monocytes (Kharbanda et al., 1995).

Mononuclear phagocyte populations are heterogeneous in number and phenotype within tumors. The populations arise through the recruitment of circulating monocytes and the differentiation of the recruited cells to factors in the tumor microenvironment. A prominent role for signaling pathways initiated through M-CSF receptor engagement has been established for regulation of primary tumor growth and metastasis (Lin et al., 2001). Given these qualities attributed to FAK and tumor associated mononuclear phagocytes, we set out to test the hypothesis that FAK regulates critical aspects of myeloid/macrophage behavior that allow these cells to support tumor progression and metastasis. 
The development of genetically engineered mouse models has added tremendously to our understanding of tumor biology (Frese et al., 2007 ). A common mechanism for tumor induction is through the expression of an oncogene or transforming element. In the initial study that characterized the MMTV-PyVmT model, primary tumors formed spontaneously in the mammary epithelium due to the directed expression of the polyoma middle $\mathrm{T}$ antigen under the control of the mouse mammary tumor virus (MMTV) long terminal repeat (LTR) (Guy et al., 1992). Outgrowth appeared at multiple foci within the mammary gland and lung tumors formed in the majority of older mice that expressed the PyVmT transgene. Transplant of primary tumors into the fat pad of normal syngeneic mice resulted in the formation of lung metastases, indicating that tumor growth in the lung was a result of a metastatic process. A study conducted a decade later addressed the early hyperplastic and mammary intraepithelial neoplasia (MIN) stages of disease (Maglione et al., 2001). Palpable masses were routinely detected by five weeks and displayed multifocal hyperplastic growth with atypia. The size and number of individual foci was heterogeneous across different zones of the inguinal mammary fat pad ductal tree. The transplant of different hyperplastic growths into the cleared mammary fat pad of syngeneic recipients resulted in differences in tumor latencies. This indicated that heterogeneity existed in the hyperplastic lesions. The model was further characterized as progressing through 4 distinct stages of disease, including hyperplasia, MIN, early carcinoma, and late carcinoma at approximately 5 , 8, 9, and 10 weeks, respectively (Lin et al., 2003). ER and PR were downregulated during this progression. Importantly, histological analysis combined with the regulated expression of cyclin $\mathrm{D} 1, \mathrm{ErbB} 2 / \mathrm{Neu}$, and $\beta 1$ integrin during these stages indicated that it served as a comparatively good model for the progression associated with human breast carcinomas.

To circumvent the problem of embryonic lethality in global FAK knockout animals, our lab utilized the Cre/LoxP system to generate a myeloid-specific conditional FAK knockout mouse in the C57B1/6 background (Clausen et al., 1999; Ye et al., 2003). The knockout results from tissue- 
specific recombination of the $F A K$ gene controlled by Cre recombinase, which is driven from the myeloid-specific promoter, LysM (FAK $\left.{ }^{\Delta \text { myeloid }}\right)$. Effective deletion in macrophages has been established at the genetic and protein levels (Figure 3.1). For my research, we generated two mouse models that allowed us to investigate whether FAK expression in myeloid linage cells regulates tumor progression. To test the role of FAK in an immune competent model, mice bearing the MMTV-PyVmT oncogene on the C57Bl/6 background were crossed with $\mathrm{FAK}^{\Delta \text { myeloid }}$ mice. To permit orthotopic implantation of human breast cancer cells, we crossed $\mathrm{FAK}^{\Delta \text { myeloid }}$ mice with severe combined immunodeficiency (SCID) mice (C57/B16 background). SCID mice lack $\mathrm{T}$ and $\mathrm{B}$ cells, but they retain normal differentiation and function of myeloid cells, antigen presenting cells, and natural killer cells (Czitrom et al., 1985; Dorshkind et al., 1985; NixonFulton et al., 1987; Phillips et al., 1989).

These mouse models were utilized in two broad capacities. First, we wanted to determine if FAK expression in myeloid lineage cells regulated tumor growth, and in the case of the PyVmT model, metastatic burden. Second, we tested FAK regulation of macrophage functions commonly attributed to TAMs.

\subsection{Materials and Methods}

3.2.1 Generation of mouse models. For the MDA-MB-231 and 231T1 orthotopic xenograft studies, WT $\left(\operatorname{LysM}^{\mathrm{wt} / \mathrm{wt}} \mathrm{FAK}^{\mathrm{fl} / \mathrm{fl}}\right)$ and $\mathrm{FAK}^{\Delta \text { myeloid }}\left(\mathrm{LysM}^{\mathrm{cre} / \mathrm{wt}} \mathrm{FAK}^{\mathrm{fl} / \mathrm{fl}}\right) \mathrm{C} 57 \mathrm{Bl} / 6$ mice were crossed with severe combined immunodeficiency (SCID) C57B1/6 mice. To confirm Cre knockin at the LysM locus, the following primers were utilized. Cre primer set: \#1

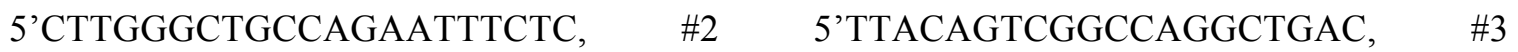
5'CCCAGAAATGCCAGATTACG (Operon). Expected products: WT LysM allele 350bp, LysM Cre allele 700bp. To confirm homozygous Prkdc (SCID) mutation, the following primers were 
Figure 3.1 Genetic recombination at the $F A K$ locus and deletion of FAK protein in myeloid lineage cells from FAK ${ }^{\Delta \text { myeloid }}$ mice. Myeloid lineage cells were immunomagnetically separated from whole bone marrow using $\alpha$-CD11b magnetic beads $\left(\mathrm{CD} 11 b^{+} \mathrm{BM}\right)$. For bone marrow derived macrophages (BMDM), whole bone marrow was cultured in media supplemented with macrophage colony stimulating factor (M-CSF) for 5 days. (A) DNA was isolated from CD11 $\mathrm{b}^{+}$ $\mathrm{BM}$ cells and BMDM from $\mathrm{WT}$ and $\mathrm{FAK}^{\Delta \text { myeloid }}$ mice and subjected to PCR using primers targeting the FAK locus. The reduction in size of the PCR fragment from $\mathrm{FAK}^{\Delta \text { myeloid }}$ mice indicates gene recombination. (B) Immunoblot of cell lysates from BMDM shows efficient loss of FAK protein in $\mathrm{FAK}^{\Delta \text { myeloid }}$ mice. 


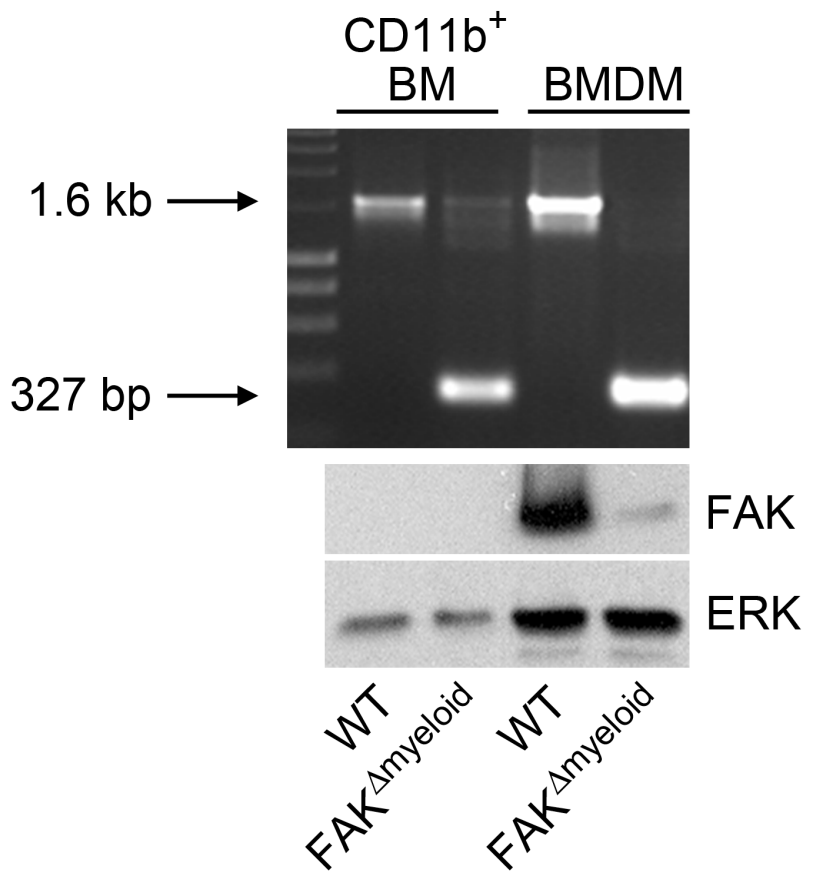


utilized. SCID primer set: \#1 5'GGAAAAGAATTGGTATCCAC, \#2 5'AGTTATAACAGCTGGGTTGGC (Operon). SCID PCR products were then subjected to restriction enzyme digest $(21 \mu \mathrm{l}$ SCID PCR product $+2.5 \mu 110 \mathrm{X}$ buffer $+1 \mu 1 \mathrm{BSA}+0.5 \mu 1$ AluI enzyme/reaction x $60 \mathrm{~min}$. at 37C) and products were resolved on 5\% agarose gel. Expected products: heterozygous SCID 38bp and 11bp, homozygous SCID $11 \mathrm{bp}$.

For the MMTV-PyVmT tumor study, WT and FAK ${ }^{\Delta \text { myeloid }}$ mice were crossed with MMTVPyVmT C57Bl/6 mice. After successful generation of WTMMTV-PyVmT $^{+/}$and $\mathrm{FAK}^{\Delta \text { myeloid }}{ }^{\mathrm{M} M T V-P y V m T}{ }^{+/-}$mice, female breeders were maintained as $\mathrm{PyVmT}^{-/-}$and males breeders as $\mathrm{PyVmT}^{+/}$.Primer set targeting PyVmT: \#1 5'TGTGCACAGCGTGTATAATCC, \#2 5'CAGAatAGgtCGGGTTGCTC (Operon). Expected product for $\mathrm{PyVmT}^{+/}$mice: $200 \mathrm{bp}$.

\subsubsection{Measurement of MMTV-PyVmT primary tumor outgrowth. Mammary glands \#1-4} (MG1-4) were palpated for tumor outgrowth. Tumor volume in MG1-4 at day 91 and d105 was determined by caliper measurement in two dimensions. Length (1) was defined as maximum attainable tumor measurement in one dimension. Width (w) was the measurement perpendicular to length. Tumor volume was derived using the formula $\left[1 \mathrm{x} \mathrm{w}^{2}\right] / 2$.

3.2.3 Tumor Histology. Immunohistochemistry (protocol from Lisa K. Gross). MG1 tumor specimens at d91 and d105 were surgically removed with forceps and scissors, fixed overnight (o/n) in $10 \%$ formalin, and transferred to $70 \%$ ethanol (ETOH). In the UVA histology core, the specimens were embedded in paraffin and sectioned onto glass microscope slides ( $5 \mu \mathrm{m}$ sections). Hematoxylin and eosin staining was completed by the core staff. For Ki67 detection by immunohistochemistry, slides were deparaffinized in xylene $(3 \times 15 \mathrm{~min}$.$) and rehydrated through$ a graded series of ethanol $\left(3 \times 30 \mathrm{sec}\right.$. in $100 \% \mathrm{ETOH}, 3 \times 30 \mathrm{sec}$. in 95\% ETOH) and $\mathrm{ddH}_{2} \mathrm{O}(3$ x $30 \mathrm{sec}$.) washes. Slides were incubated in peroxide solution $\left(0.3 \% \mathrm{v} / \mathrm{v} \mathrm{ddH}_{2} \mathrm{O}\right)$ x $30 \mathrm{~min}$. to block endogenous peroxidase activity. For antigen retrieval, the slides were placed in $10 \mathrm{mM}$ 
citrate buffer pH6.0 $\left(9 \mathrm{ml}\right.$ of $.1 \mathrm{M}$ citric acid $+41 \mathrm{ml} 0.1 \mathrm{M}$ sodium citrate $\left.+450 \mathrm{ml} \mathrm{ddH}_{2} \mathrm{O}\right)$ and heated in microwave $4 \times 5 \mathrm{~min}$. at high setting. After each $5 \mathrm{~min}$. pulse, the reservoir was q.s. with $\mathrm{ddH}_{2} \mathrm{O}$. The slides were cooled at RT ( $15-60 \mathrm{~min}$.), and transferred to PBS. A contiguous hydrophobic barrier was drawn around sections with an Elite PAP pen (DBS). To block endogenous biotin (Vector), sections were incubated with avidin for 10 min., washed with PBS, incubated with biotin for 10 min., and washed in PBS. For blocking and antigen detection, $\alpha$-rat IgG kit (Vector) was utilized. Blocking solution was made by mixing 3 drops of stock rabbit serum in $3 \mathrm{ml}$ PBS. Biotinylated secondary antibody solution was made by mixing 3 drops of rabbit serum with 1 drop $\alpha$-rat IgG biotinylated secondary antibody in $10 \mathrm{ml}$ PBS. Elite ABC reagent was prepared by mixing 2 drops of Reagent A with 2 drops of Reagent B in $5 \mathrm{ml}$ PBS. The sections were blocked for 10min., incubated at RT for 60 min. in $\alpha$-Ki67 [1:100 blocking solution] (DAKO), washed in PBS, incubated with secondary antibody (10 min. at RT), washed in PBS, incubated with $\mathrm{ABC}$ reagent (10 min. at $\mathrm{RT})$, and washed. $\mathrm{DAB}$ working solution (Vector SK-4100) was prepared following manufacturers protocol and applied to sections for 7 min. Following a $5 \mathrm{~min}$. wash in running tap water, sections were stained with hematoxylin for $\sim 45$ sec., washed again in tap water, and dehydrated through a series of washes $(3 \times 10$ sec. ddH $_{2} \mathrm{O}$ ), 3 X 10 sec. 95\% ETOH, 3 X 10sec. 100\% ETOH, 3 x 10 sec. xylene). The sections were then mounted with cytoseal and air dried. Images were acquired on the Olympus high magnification scope in the UVA Advanced Microscopy core facility. Immunofluorescence. Day 105 MG1 tumors were flash frozen in a slurry of dry ice $+100 \% \mathrm{ETOH}$, stored at $-80 \mathrm{C}$, and sectioned by the UVA histology core. Staining protocol was adapted from Engelhard Lab (Liz Thompson). Sections were fixed in acetone at -20C x 10 min., air dried, encircled with PAP pen, washed in PBS, blocked with $\alpha-C D 16 / 32$ (eBioscience 14-0161-85) [1/1000 PBS $+5 \%$ BSA] $x$ 15 min., washed in PBS, incubated with avidin [1 drop/200 $\mu 1$ PBS + 5\% BSA] x 15 min.,

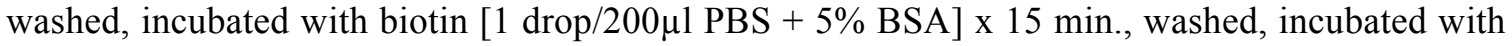
$\alpha$-CD31 (abcam) $[1: 100$ PBS $+5 \%$ BSA $]$ x 60min. at RT, washed 2 x PBS, incubated with $\alpha$-rat 
Texas Red (Jackson Lab) [1:300 in PBS $+5 \%$ BSA] x 30min. at RT, washed 3 x PBS, and mounted with ProlongGold + DAPI (Life technologies). Images were acquired on the Olympus high magnification microscope.

\subsubsection{Quantification of vessel density in day 105 tumor specimens. Images of $\alpha$-CD31} TexasRed stained tumor sections were converted in Image J software (NIH) to 8 bit black and white images. A threshold was set and applied to all images, and the percentage of each image that was positive for CD31 staining was determined by Image $J$. The values for all images from a given tumor specimen were compiled and averaged.

3.2.5 RTPCR quantification of PyVmT transcript in the lung. Lung specimens from day 105 $\mathrm{PyVmT}^{+/-}$mice were harvested and frozen at $-80 \mathrm{C}$ in $1 \mathrm{ml} \mathrm{RNA}$ later solution. At time of analysis, the specimens were thawed at RT and a sample $\sim .025-.03 \mathrm{mg}\left(\sim 3 \mathrm{~mm}^{3}\right)$ was removed and placed in RLT buffer + BME $(600 \mu 1)$. The unused portion was returned to $-80 \mathrm{C}$. The specimens were homogenized using a rotor-stator homogenizor and RNA was purified (RNeasy mini kit, Qiagen)

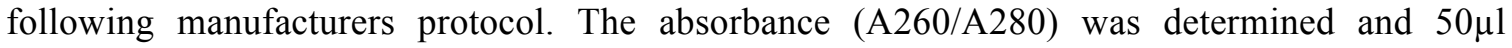
solutions [10ng RNA/5 $\mu 1 \mathrm{ddH}_{2} \mathrm{O}$ ] were prepared for RTPCR analysis. First strand synthesis was completed using random primers. Primer sets targeting GAPDH and PyVmT were utilized for RTPCR (Operon), and samples were run in duplicate. GAPDH \#1 5'GACCCCTTCATTGACCTCAAC， \#2 5'CTTCTCCATGGTGGTGAAGA; PyVmT \#1 5'GGGGAtATGCTGtCATCGGGCTCA， \#2 AGCTACCAGTCGCCGCCTAAGA. SYBR Green was utilized for detection. The RTPCR units for PyVmT transcript were normalized to the units obtained for GAPDH.

3.2.6 Determination of lung metastatic burden by histology. Lungs were harvested from WT and $\mathrm{FAK}^{\Delta \text { myeloid }}$ mice with forceps and scissors, fixed in $10 \%$ formalin $\mathrm{o} / \mathrm{n}$, transferred to $70 \%$ ETOH, paraffin embedded, sectioned $(5 \mu \mathrm{m})$, and stained with hematoxylin and eosin (H\&E). 
Photographs were compiled for the entire histological section from each specimen using the Olympus high magnification microscope ( $4 \mathrm{X}$ objective). The perimeter of tumor regions and whole lung region in each photograph were traced using Image $\mathbf{J}$ software. Metastatic tumor burden was defined as the percentage of tumor surface area relative to the total lung surface area. The number of metastatic foci for each sectioned was determined by counting.

3.2.7 Generation of 231T1 cell line. The protocol was adapted from Jessani et al. $1.0 \times 10^{6}$ MDA-MB-231 cells (Ameican Type Tissue Culture (ATCC)) were prepared in 100 $\mu 1$ Matrigel (BD Biosciences), implanted into MG4, and monitored for outgrowth. At day 92 (tumor size $7.56 \mathrm{~mm} \times 7.10 \mathrm{~mm}$ ), the tumor was removed with forceps and scissors, rinsed with PBS, diced with scissors, and plated in $10 \mathrm{~cm}$ tissue culture dish in $10 \mathrm{ml}$ complete media. The culture was washed at day 3 and media was replenished. By day 4, small colonies started to form. The media was replenished every several days until the first passage at day 25 post-tumor harvest. The new cells were designated $231 \mathrm{~T} 1$.

\subsubsection{In vitro induction of macrophage polarizarion. Day 4 BMDM were trypanized,} quenched, enumerated, and plated in $10 \mathrm{~cm}$ petri dishes at $1.0 \times 10^{6}$ cells/dish in BMDM complete media. The following day, the adherent cells were washed and replenished with either media alone, media + IFN- $\gamma$ [10ng/ml] + LPS [100ng/ml], or media + IL-4 [20ng/ml] for 24hrs. Griess reaction (M1 polarization). One $\mathrm{ml}$ conditioned media $(\mathrm{CM})$ was harvested and centrifuged (4000rpm x $7 \mathrm{~min}$.) to pool suspended debris. In a spectrophotometer cuvette, $500 \mu 1$ Griess reagent (Sigma) was added to $500 \mu \mathrm{l} \mathrm{CM}$. After a $15 \mathrm{~min}$. incubation at $\mathrm{RT}$, the absorbance at 540nm (A540) was determined for each sample. Solutions of known nitrite concentrations were run concurrently and a linear standard curve was established (A540 as a function of $\mu \mathrm{M}$ nitrite). The concentration of nitrite in the CM was calculated utilizing the slope of this line and the A540 measurements. The nitrite concentration of each sample was normalized to the protein concentration of the cell lysates, as determined by BCA method. Cell lysates were prepared in 
$150 \mu 1$ RIPA buffer and subjected to SDS/PAGE and immunoblot detection. CD206 expression (M2 polarization). For detection of CD206 by immunoblot, cell lysates from media alone and media + IL-4 treated BMDM were prepared in sample buffer without $\beta$-mercaptoethanol (BME), incubated o/n at $4{ }^{\circ} \mathrm{C}$ with $\alpha-\mathrm{CD} 206$ (AbD Serotec) $[1: 20005 \%$ milk $\}$, and incubated with $\alpha$-rat HRP [1:5000 5\% milk] x 45 min. For CD206 immunofluorescence, BMDM were plated on fibronectin (Sigma) coated glass coverslips and allowed to adhere o/n. The following day, the adherent cells were washed with PBS, fixed with 3\% paraformaldehyde x 20 min. at RT, washed, permeabilized with $0.4 \%$ Triton X100 x 6 min. at RT, washed, blocked with $10 \%$ BSA/PBS x 30min., incubated with $\alpha$-CD206 FITC (AbD Serotec), washed, and mounted with Prolong Gold + DAPI. Images were acquired on the Olympus high magnification microscope in the advanced microscopy core at UVA.

3.2.9 Analysis of BMDM for pro-MMP9 expression. $1.0 \times 10^{6}$ bone marrow derived macrophages (BMDM) from WT and $\mathrm{FAK}^{\Delta \text { myeloid }}$ mice were plated in a $10 \mathrm{~cm}$ tissue culture (t.c.) dish in BMDM media and cultured o/n. The following day (20 hrs. post-plating), the conditioned media $(\mathrm{CM})$ was harvested, centrifuged at $4000 \mathrm{rpm}$ to pool debris, and concentrated with $10 \mathrm{kDa}$ centrifugal filters (Amicon). The retentate volume was determined by pipet and an equivalent proportion of each sample was prepared for zymography. Samples were prepared in non-reducing sample buffer without bromophenol blue dye and resolved on $8 \%$ SDS/PAGE $+0.33 \mathrm{mg} / \mathrm{ml}$ type A porcine gelatin (Sigma). The gel was washed $2 \times 30 \mathrm{~min}$. in $2.5 \% \mathrm{TX} 100 / \mathrm{ddh}_{2} 0$ and placed in reaction buffer $(20 \mathrm{mM}$ Tris $5 \mathrm{mM} \mathrm{CaCl} 2 \mathrm{pH} 7.4) \mathrm{o} / \mathrm{n}$. The following day, the gel was stained in $0.25 \%$ Coomassie $/ 40 \%$ isopropanol for $60 \mathrm{~min}$, then destained in $7 \%$ acetic acid. The gel was imaged and the intensity of signal in each lane (of inverse image generated in Photoshop) was determined by densitometry. The signal was normalized to ERK expression as determined by immunoblot. The adherent cells were washed, $150 \mu 1.1 \%$ TX100 + protease inhibitors (PI) lysis buffer was added to the plate, and the cells were collected by scraping. The lysates were 
incubated for $60 \mathrm{~min}$. on ice (with intermittent vortex), centrifuged at $13000 \mathrm{rpm}$ x 1 5min., and the supernatant was retained and frozen. Equivalent volume of lysate as prepared for each sample and resolved on $8 \% \mathrm{SDS} / \mathrm{PAGE}$ under reducing conditions. The gel was transferred to nitrocellulose, blocked for 20 min. (5\%milk), and incubated with $\alpha$-MMP9 (abcam) 1:2000 and $\alpha$ ERK 1:2000 o/n $4^{\circ} \mathrm{C}$. The next day, the membranes were washed, incubated with $\alpha$-rabbitHRP (MMP9 1:10,000, ERK 1:5000) for 60 min., washed, incubated with ECL reagents, and exposed to film. Signal intensity was determined by densitometry.

3.2.10 MDA-MB-231 and 231T1 tumor outgrowth. Subconfluent MDA-MB-231 or 231T1 cells were trypsanized, quenched, and enumerated. Cell solutions were prepared at $4.0 \times 10^{6}$ cells/100 $\mu 1$ and loaded into $1 \mathrm{ml}$ syringes (27 gauge/1.25 inch needle). Mice were anesthetized with tri-bromoethanol $(\sim 250 \mathrm{mg} / \mathrm{Kg})$ and $50 \mu 1$ of cell solution was implanted into the right (R) and left (L) MG4. Mice were palpated for growth. Tumor volume was determined as described for the MMTV-PyVmT model.

\subsection{Results}

\subsubsection{FAK expression in myeloid lineage cells regulates early stages of tumor initiation/growth but not later stage growth or vascularity}

A hallmark of the MMTV-PyVmT model is $100 \%$ penetrance in primary tumor outgrowth (Guy et al., 1992). We utilized this quality to determine if the loss of FAK in myeloid lineage cells regulated tumor initiation. To accomplish this, mammary glands 1-4 in WT and FAK ${ }^{\Delta \text { myeloid }}$ mice were palpated to detect tumor growth (Figure 3.2A). Initiation was defined as the number of days post-birth that a mass was first detected by palpation. Our results indicated that FAK did not

regulate tumor initiation, as there was no difference between WT and $\mathrm{FAK}^{\Delta \text { myeloid }}$ mice in the time to appearance of the first detectable mass in any mammary gland (MG) (Figure 3.2B). However, 
Figure 3.2 Tumor initiation in the MMTV-PyVmT murine breast carcinoma model is not regulated by FAK in myeloid lineage cells. Initiation of tumor growth in mammary glands 2-4 (MG2-4) is significantly delayed relative to MG1. (A) Schematic of mouse indicating the location of mammary gland (MG)1 - MG4. (B) MG1-4 in WT and FAK $\Delta$ myeloid mice harboring the PyVmT oncogene were palpated for growth. Schematic illustrates genetic profile of WT and $\mathrm{FAK}^{\Delta \text { myeloid }}$ mice and range of first detectable tumor mass. Graph depicts the time to tumor initiation in the individual mammary glands. Data are representative of WT: 79 MG1, 74 MG2, 62 MG3, 66 MG4; FAK ${ }^{\text {myeloid: }} 118$ MG1, 119 MG2, 101 MG3, 100 MG4. SEM, * = P < .05. Mouse diagram adapted from Jackson Labs. 
A.

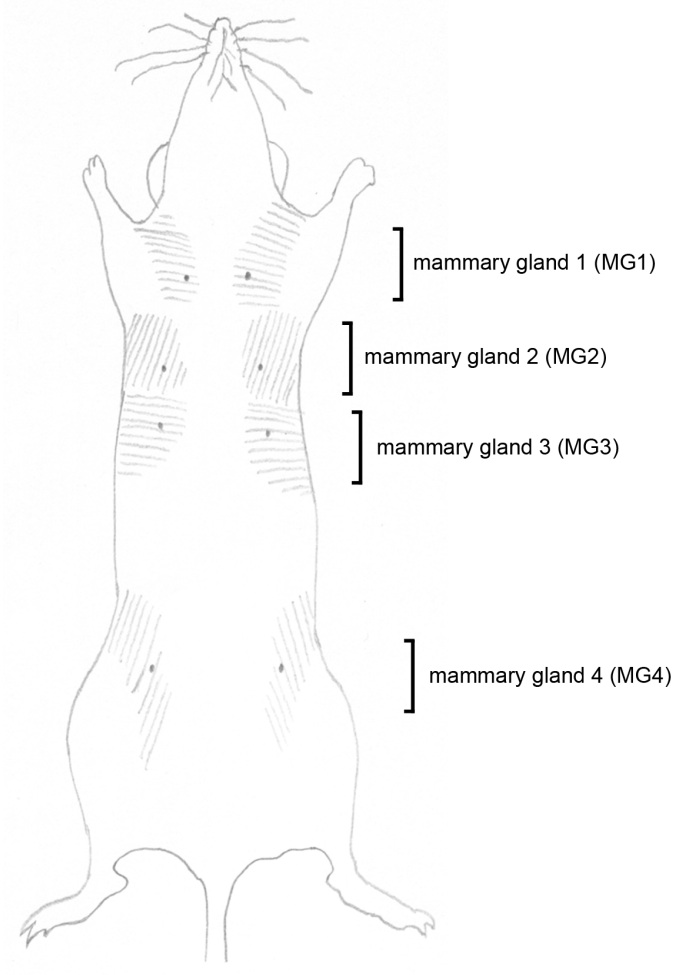

B.

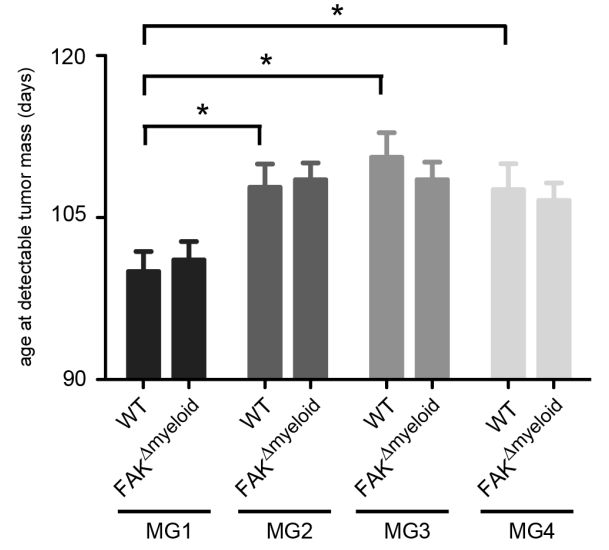


tumors in MG1 were detected significantly earlier relative to the other three MG. Previous reports showed that the mean tumor latency on the C57Bl/6 background was 92 days (Davie et al., 2007).

We next wanted to determine if FAK regulated the growth of primary tumors. Mammary gland tumors were measured by caliper in two dimensions (length ([1] and width [w]) at day 91 (week 13) and volume was assigned based on an established formula $\left(\left[1 \mathrm{x} \mathrm{w}^{2}\right] / 2\right)$. A comparison of burden in MG 2-4 showed no difference between WT and FAK ${ }^{\Delta \text { myeloid }}$ mice (Figure 3.3A). However, MG1 tumors in FAK ${ }^{\Delta \text { myeloid }}$ mice were significantly larger compared to WT mice. Histological analysis of tumor outgrowth within the mammary fat pad (Figure 3.3B) and the mixed peripheral and scattered location of $\mathrm{Ki}^{+} 7^{+}$cells in the tumor lesions (Figure 3.3C) indicated that day 91 tumors were at the MIN/early carcinoma stage. Analysis of primary tumors two weeks later (day 105) again revealed a roughly two-fold difference in MG1 burden (Figure 3.4A). Tumors from both cohorts showed an increased proportion of tumor mass at this time period that was highly disorganized, indicating that MG1 had progressed in stage (Figure 3.4B). When combined with our results at day 91, these data indicated that FAK expression in myeloid cells functioned to either inhibit the early stages of tumor progression or loss of FAK in these cells promoted this process. Once the tumors became established, however, FAK expression in myeloid cells appeared not to have any additional control over tumor growth.

Given that MG1 tumors at d105 were significantly larger in FAK ${ }^{\Delta \text { myeloid }}$ mice, and macrophages had been shown to regulate vessel density and the angiogenic switch in this model, we wanted to determine if vascularity was enhanced with the loss of FAK (Lin et al., 2006). MG1 tumors were harvested, frozen, and sectioned for immunofluorescence staining with antibody targeting CD31, a marker for endothelial cells. Regions of each section that showed a high degree of tumor mass, as indicated by concentrated nuclear DAPI staining, were analyzed (Figure 3.5A). The area of the tumor comprised of vasculature was determined by Image J software (NIH) relative to the total tumor area for each microscopic field. Multiple fields spanning the entire tumor section were 
Figure 3.3 MG1 tumors at day 91 are significantly larger in FAK ${ }^{\Delta \text { myeloid }}$ mice relative to WT mice and display characteristics indicative mammary intraepithelial neoplasia (MIN)/ early carcinoma tumor stage. (A) Tumor growth in MG1-4 at day 91 was determined by caliper measurement in two dimensions. Length (1) was defined as maximum attainable tumor measurement in one dimension. Width (w) was the measurement perpendicular to length. Tumor volume was derived using the formula $\left[1 \mathrm{x} \mathrm{w}^{2}\right] / 2$. Day $91 \mathrm{MG} 1$ tumors were surgically removed, fixed, embedded in paraffin, and sectioned. Sections from WT and FAK ${ }^{\Delta \text { myeloid }}$ mice were stained with hematoxylin \& eosin (H\&E) (B) or antibody recognizing Ki67 (C). H\&E stained sections highlight tumor foci intermixed with mammary gland tissue. Ki67 staining in tumor cells indicates proliferating tumor cells located throughout foci. H\&E magnification $4 \mathrm{X}$ top, $20 \mathrm{X}$ bottom. Ki67 magnification 20X top, 40X bottom. Scale bar $200 \mu \mathrm{m}(4 \mathrm{X}), 50 \mu \mathrm{m}(20 \mathrm{X}), 20 \mu \mathrm{m}$ (40X). SEM, $*=\mathrm{P}<.05$. 
A.

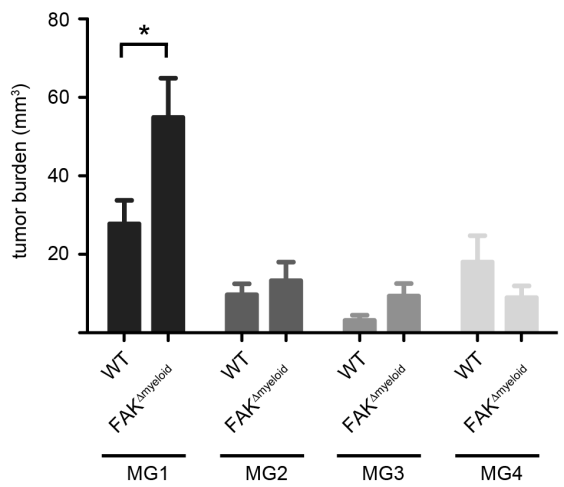

B.

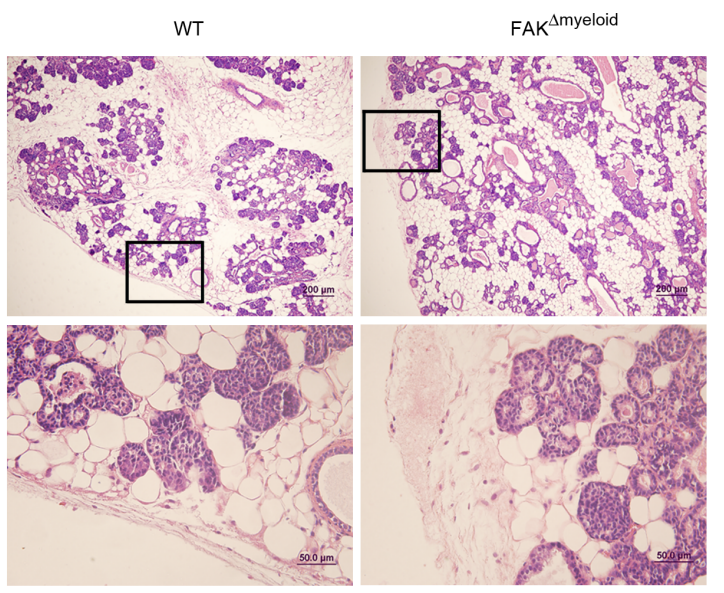

Hematoxylin \& Eosin
C.

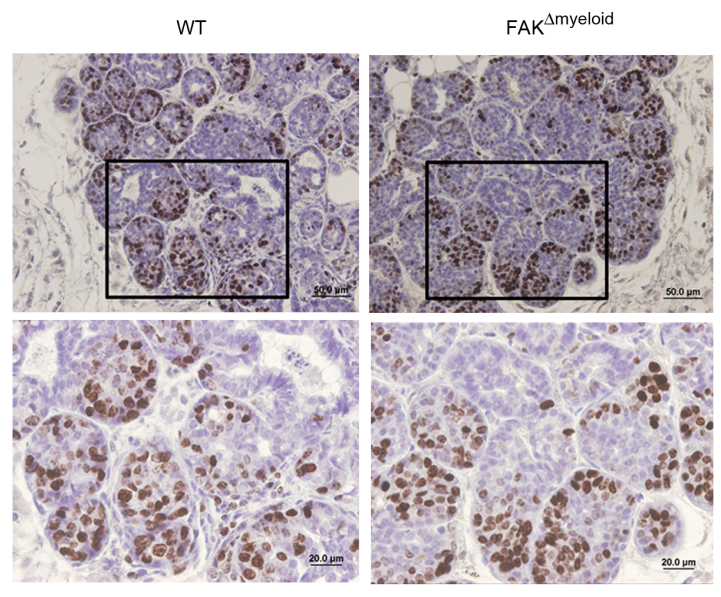

Ki67 
Figure 3.4 MG1 tumor burden at day 105 is significantly greater in FAK ${ }^{\text {Amyeloid }}$ mice relative to WT mice and displays hallmark features of advanced disease. (A) MG1-4 tumor volumes were determined by caliper measurement. (B) Day 105 tumor specimens were stained with H\&E. Extreme disorganization in tissue architecture and tumor growth are visible throughout mammary gland. Magnification 4X top, 20X bottom. Scale bar 200um (4X), 50um (20X). SEM, $*=P<.05$. 
A.

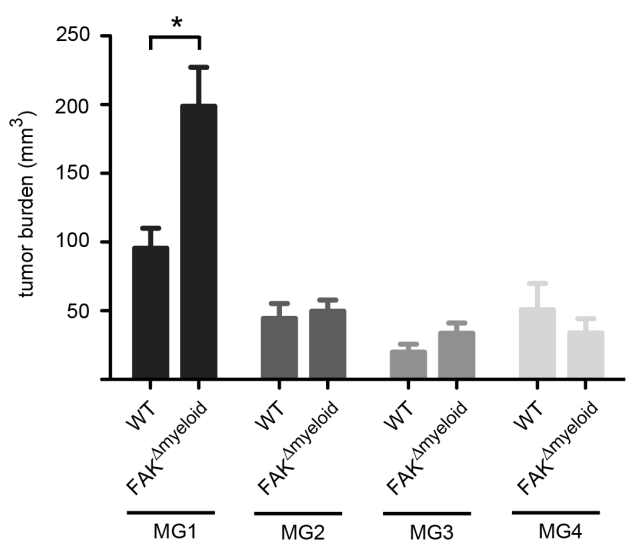

B.
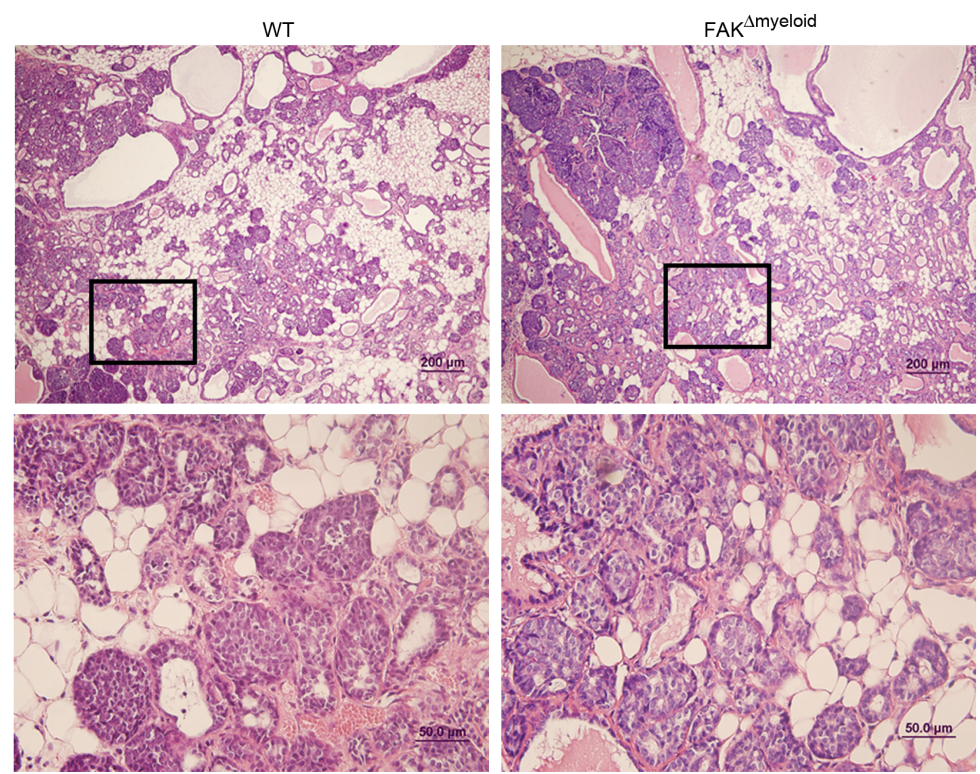

Hematoxylin \& Eosin 
Figure 3.5 Vessel density is equivalent in day 105 MG1 tumors from WT and FAK Mmyeloid $^{\text {A }}$ mice. (A) Tumors were surgically removed, frozen, and sectioned. Antibody targeting the endothelial cell antigen CD31 was utilized to delineate vasculature during immunofluorescent staining of the tumor specimens. DAPI was added during specimen mounting to identify nuclei of individual cells. Stained sections were photographed and then analyzed using Image J software (NIH). Photographs are representative examples of DAPI (left panel, blue) and CD31 (center, red) staining of a region within MG1 tumors. Far right panel illustrates CD31 positive staining (white) following conversion in Image $\mathrm{J}$. The percentage of $\mathrm{CD} 31^{+}$area of the total field was determined by Image J. Multiple fields were analyzed for each tumor sections. (B) Graph depicting the percentage of tumor area that is $\mathrm{CD} 31^{+}$. Individual bars represent the measurements of all fields analyzed for a given tumor specimen. Data are representative of 6 WT tumors and 14 FAK $^{\Delta \text { myeloid }}$ tumors. (C) The graph displays the SEM values of each tumor specimen (bar) from (B). 
A.

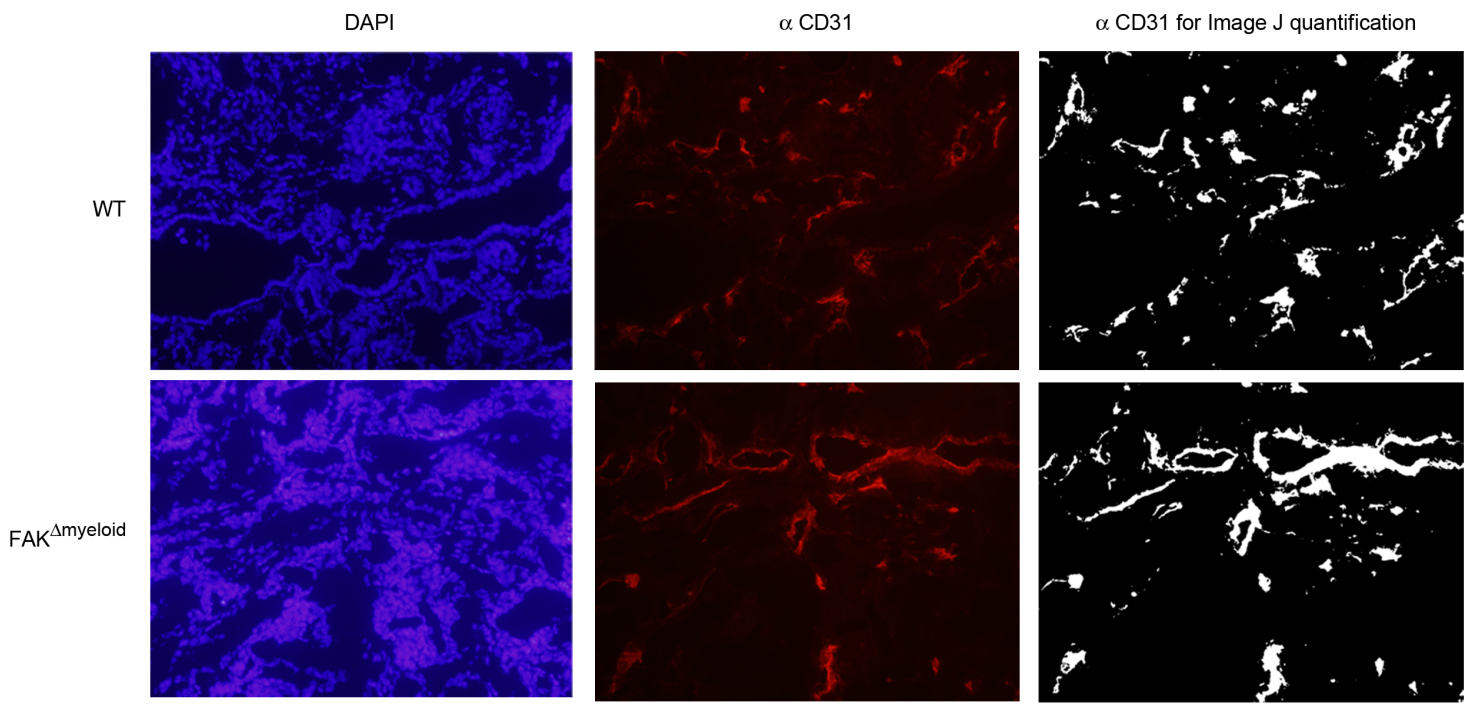

B.

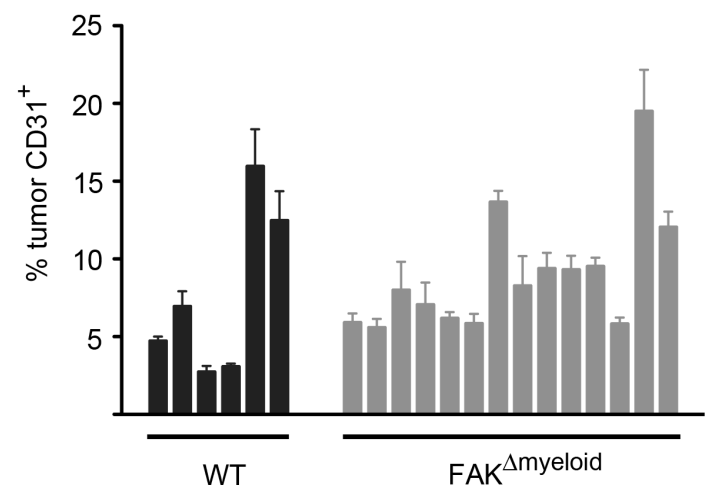

C.

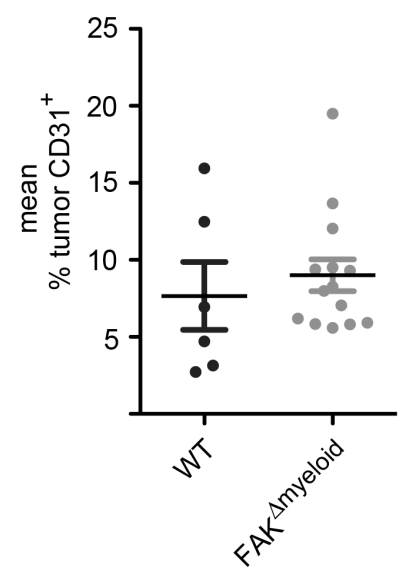


analyzed by this method and the values obtained for each image were compiled (Figure 3.5B and C). Our results indicated that vessel density in MG1 at day 105 was not regulated by FAK expression.

\subsubsection{FAK does not control macrophage behavior critical for lung metastasis}

In addition to regulating the angiogenic switch, macrophages are also required for progression to carcinoma and metastasis to the lung (Lin et al., 2001). Therefore, we wanted to determine if FAK regulated tumor metastasis to the lungs of MMTV-PyVmT mice. To accomplish this, we utilized complementary methods. First, lungs were harvested from a cohort of WT and $\mathrm{FAK}^{\Delta \text { myeloid }}$ mice (age 98-163), fixed, embedded in paraffin, sectioned, and stained with H\&E (Figure 3.6A). Tumor nodules on the lung were readily evident by microscopic examination of these sections (Figure 3.6B). Photographic images of the entire lung section were compiled. Tumor and lung surface areas were determined by tracing around the representative areas using Image $\mathrm{J}$ software. Metastatic tumor burden was defined as the percentage of tumor surface area relative to the total lung surface area for each section. As expected, there was a positive correlation between age and metastatic burden for WT mice, and $\mathrm{FAK}^{\Delta \text { myeloid }}$ mice displayed a positive correlation as well (Figure 3.6C). However, there was no difference in the percentage of tumor surface area between the two groups or the number of metastatic foci (Figure 3.6D).

Given that the age of mice varied considerably for both groups, we next analyzed metastatic burden in mice at day 105 by quantifying the level of PyV transcript in the lungs. Quantification of GAPDH transcript allowed normalization across samples. We first wanted to establish that our primer set specifically targeted $\mathrm{PyV}$ transcript. As expected, PyV transcript was detected in primary tumor and lung that contained metastatic growth, with the level in the primary tumor comparatively much greater (Figure 3.7A). Analysis of lung from $\mathrm{WT} \mathrm{PyV}^{-/}$mice showed negligible PyV transcript, indicating that the primer set targeted PyV. Using this method, we saw 
Figure 3.6 Metastatic tumor burden is equivalent in WT and FAK ${ }^{\Delta \text { myeloid }}$ mice. Lungs were harvested from WT and $\mathrm{FAK}^{\Delta \text { myeloid }}$ mice, fixed, paraffin embedded, sectioned, and stained with H\&E. (A) Schematic illustrates the range of age at lung harvest. Graph represents age of individual mice at harvest. Data are representative of $31 \mathrm{WT}, 36 \mathrm{FAK}^{\Delta \text { myeloid }}$. (B) Schematic of lung processing. Dotted line at top indicates plane of lung section, followed by pictorial of lung cross section. Dotted box on pictorial is depicted in photographs of representative WT and $\mathrm{FAK}^{\Delta \text { myeloid }}$ lung specimens. Arrows indicate metastatic tumor nodules. (C) Photographs were compiled for the entire histological section. The perimeter of tumor regions and whole lung region in each photograph were traced using Image $\mathbf{J}$ software. Metastatic tumor burden was defined as the percentage of tumor surface area relative to the total lung surface area. Dot plots represent lung tumor burden as a function of age. A best fit regression line is indicated on each graph $\left(\mathrm{R}^{2}=.12(\mathrm{WT}), .05\left(\mathrm{FAK}^{\Delta \text { myeloid }}\right)\right)$. (D) Graphs represent tumor burden as the percentage of lung with tumor growth (left) and the number of metastatic nodules per section (right). (E) Graphs represent metastatic tumor burden as a function of primary tumor burden. 
A.

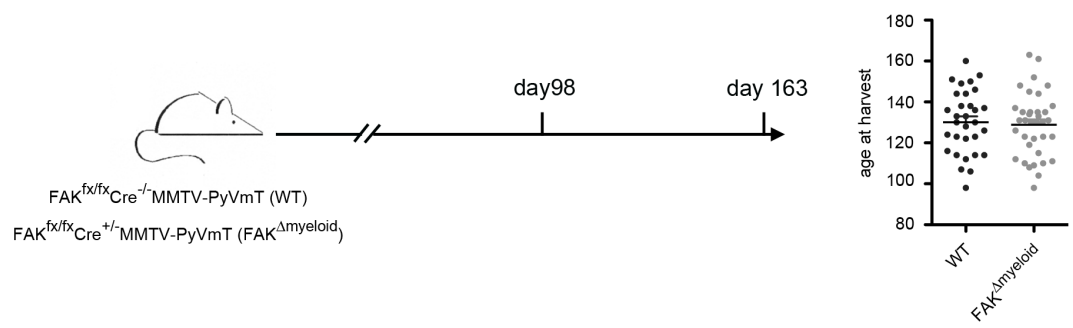

B.

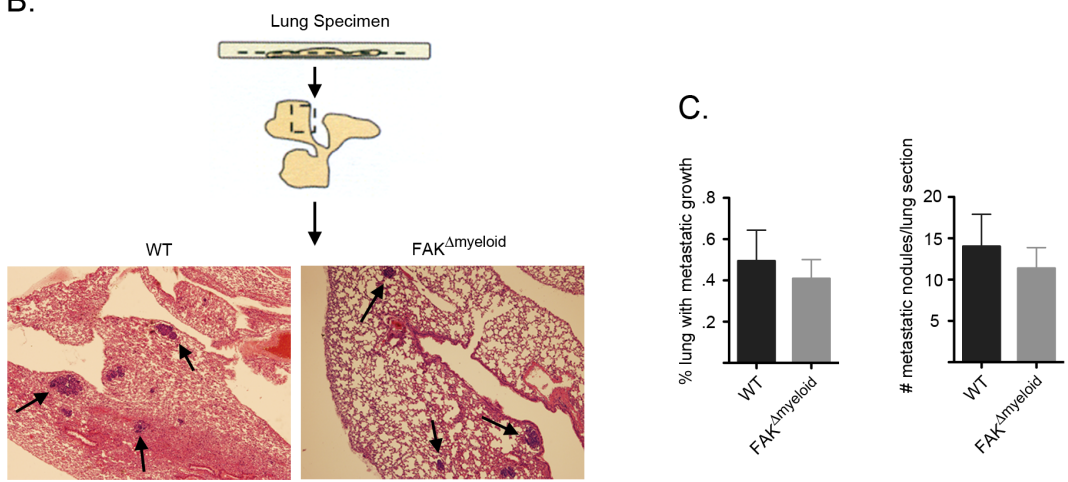

D.
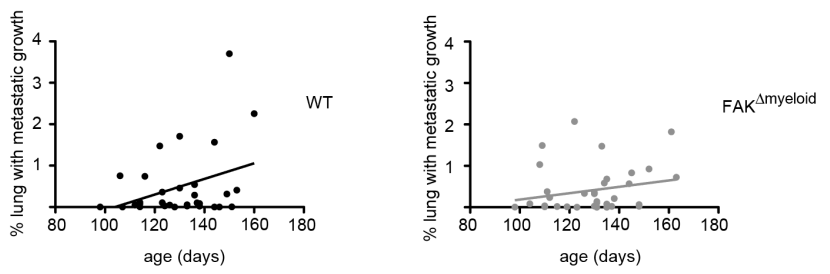

E.
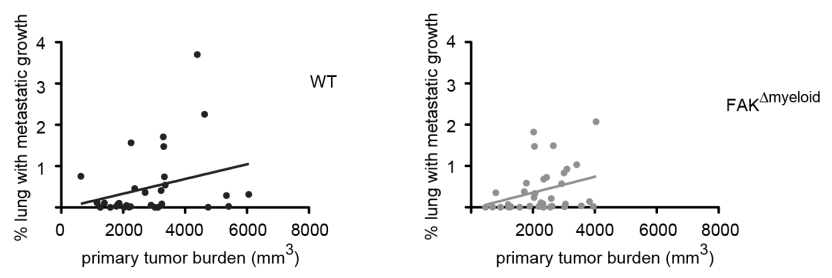
Figure 3.7 PyVmT transcript levels in the lungs of day $105 \mathrm{WT}$ and FAK ${ }^{\text {dmyeloid }}$ mice are equivalent. (A) RNA was extracted from three tissues, lung from $\mathrm{PyVmT}^{+/-}$mouse that exhibited metastatic growth (column 1), primary tumor from $\mathrm{PyVmT}^{+/-}$mouse (column 2), and lung from WT PyVmT ${ }^{--}$mouse (column 3), and subjected to RTPCR targeting PyVmT transcript. All values were normalized to transcript levels of GAPDH. (B) RNA was extracted from WT and FAK $^{\Delta \text { myeloid }}$ mice lung specimens of day 105 mice and analyzed for PyVmT transcript by RTPCR. All values were normalized to GAPDH transcript. Data are representative of 8 WT and 10 FAK $^{\Delta \text { myeloid }}$ mice. 
A.

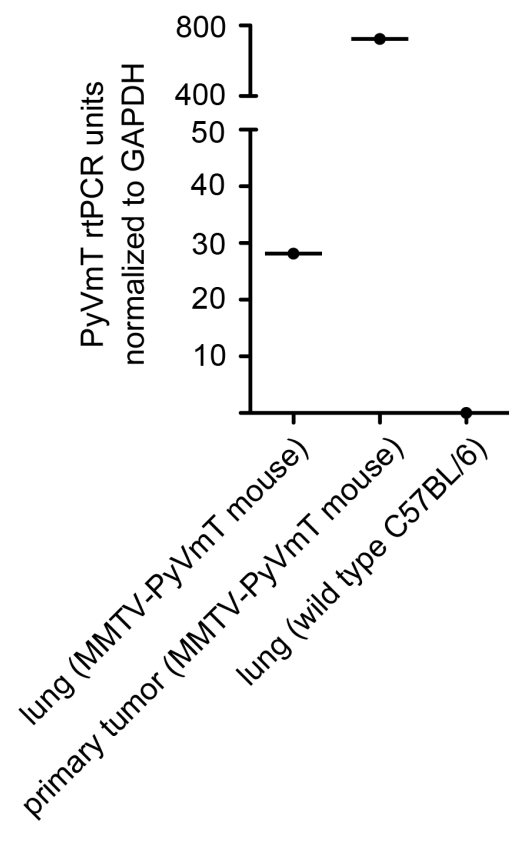

B.

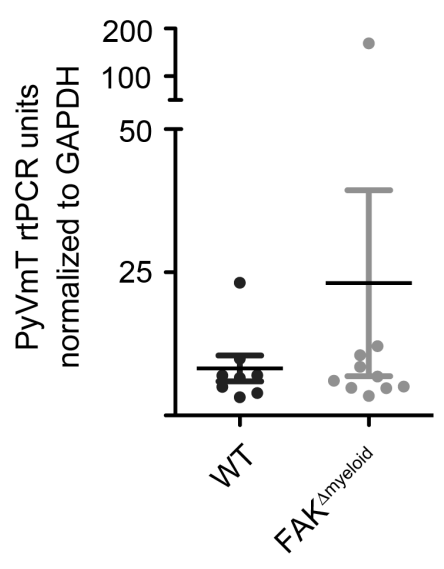


no difference in metastatic burden between WT and $\mathrm{FAK}^{\Delta \text { myeloid }}$ mice (Figure 3.7B). Our combined results from histological examination and RTPCR indicated that FAK expression in myeloid lineage cells does not regulate metastasis in the MMTV-PyVmT model.

\subsubsection{FAK does not regulate macrophage polarization in response to classical Th1 and Th2 cytokines}

To this point, our data indicated that the loss of FAK in myeloid lineage cells resulted in larger MG1 tumors but did not regulate events important for metastasis. This suggested that FAK was involved in potentiating a microenvironment that opposed tumor growth and/or blocking functions that contributed to tumor growth. This could be due to a myriad of factors considering the heterogeneity of the tumor microenvironment. It is well established that macrophages can polarize to an inflammatory (M1) or regenerative/growth (M2) activation state in response to stimuli associated with a Th1 or Th2 response, respectively (Gordon 2003). In the tumor microenvironment, the genetic signature and protein expression profile of M2 TAM are protumor. In the MMTV-PyVmT model, it was revealed that Th2 cytokines produced by $\mathrm{CD}^{+} \mathrm{T}$ lymphocytes drive TAM to an M2 activation state (DeNardo et al., 2009). Concomitant with this transition was an enhanced invasive phenotype. The presence of $\mathrm{CD}^{+}$cells did not impact primary tumor growth or the accumulation of $\mathrm{CD} 11 \mathrm{~b}^{+} \mathrm{Gr}-1^{-} \mathrm{F} 4 / 80^{+}$macrophages or $\mathrm{CD} 11 \mathrm{~b}^{+} \mathrm{Gr}-$

$1^{\text {high }} \mathrm{F} 4 / 80^{-}$immature myeloid cells. These data suggested that interplay between the adaptive and innate immune cells in the PyVmT model modifies TAM effector function through the production of factors by CD4 T lymphocytes.

To determine if FAK controlled the ability of macrophages to polarize to an antitumor/inflammatory (M1) and/or pro-tumor/growth (M2) state, we treated bone marrow-derived macrophages (BMDM) from WT and $\mathrm{FAK}^{\Delta \text { myeloid }}$ mice with the classical M1 (IFN- $\gamma+$ lipopolysaccharide (LPS)) or M2 (IL-4) polarizing factors. Enhanced nitric oxide (NO) 
production by macrophages in response to IFN- $\gamma+$ LPS is an indicatior of M1 polarity. The quantification of secreted nitrite using the Griess reaction serves as a surrogate measure for this response. Analysis of conditioned media in IFN- $\gamma+$ LPS-treated BMDM indicated that nitrite

levels were significantly greater than in untreated cells, however both WT and FAK ${ }^{\Delta \text { myeloid }}$ BMDM showed a similar degree of increased production (Figure 3.8). Levels of nitrite in IL-4 treated and untreated BMDM were comparable. This indicated that FAK did not regulate the ability of macrophages to polarize toward an M1 phenotype in vitro in response to IFN- $\gamma+$ LPS treatment. Expression of the scavenger receptor CD206 is upregulated on M2 macrophages. Utilizing immunofluorescence and immunoblot, we detected a significant increase in CD206 expression in IL-4 treated BMDM (Figure 3.9A and B). However, CD206 expression in WT and $\mathrm{FAK}^{\Delta \text { myeloid }} \mathrm{BMDM}$ appeared to be similar, suggesting that FAK also did not regulate macrophage polarization toward an M2 phenotype in response to IL-4.

\subsubsection{FAK controls the compartmentalization of pro-MMP-9}

Matrix metalloproteinases (MMP) have been implicated in the potentiation of tumor progression, primarily through enhancement of an invasive phenotype (Noel et al., 2008; Kessenbrock, et al., 2010; Egeblad et al., 2002). The MMP family is large, and production of MMP is attributed to numerous cell types, including fibroblasts, endothelial cells, lymphocytes, neutrophils, and macrophages among others. Enzymatic activity is regulated by gene expression, association with specific inhibitors, or proteolytic cleavage of the full length inactive pro-enzyme. In addition, the enzymes can be compartmentalized through integrin interactions. This confers regulation by localizing MMP activity to the pericellular microenvironment. Activation of MMP occurs extracellularly and subsequent to the secretion of the inactive pro-enzyme (Fridman et al., 2003). MMP-9 has received significant attention in the context of tumor growth and vessel development. In a murine breast carcinoma model, MMP-9 production from $\mathrm{CD} 11 \mathrm{~b}^{+}$myelomonocytic cells was essential for vasculogenesis and tumor growth (Ahn and Brown, 2008). It has been shown in 
Figure 3.8 Macrophage polarization by INF- $\gamma+$ LPS treatment is not regulated by FAK. Bone marrow derived macrophages were treated for 24 hours with IFN- $\gamma[10 \mathrm{ng} / \mathrm{ml}]+$ LPS $[100 \mathrm{ng} / \mathrm{ml}]$ or IL-4 $[20 \mathrm{ng} / \mathrm{ml}]$, at which time nitrite levels in the conditioned media were determined by the Griess reaction. M1 polarized macrophages display enhanced nitrite secretion. Data are representative of three independent experiments. 


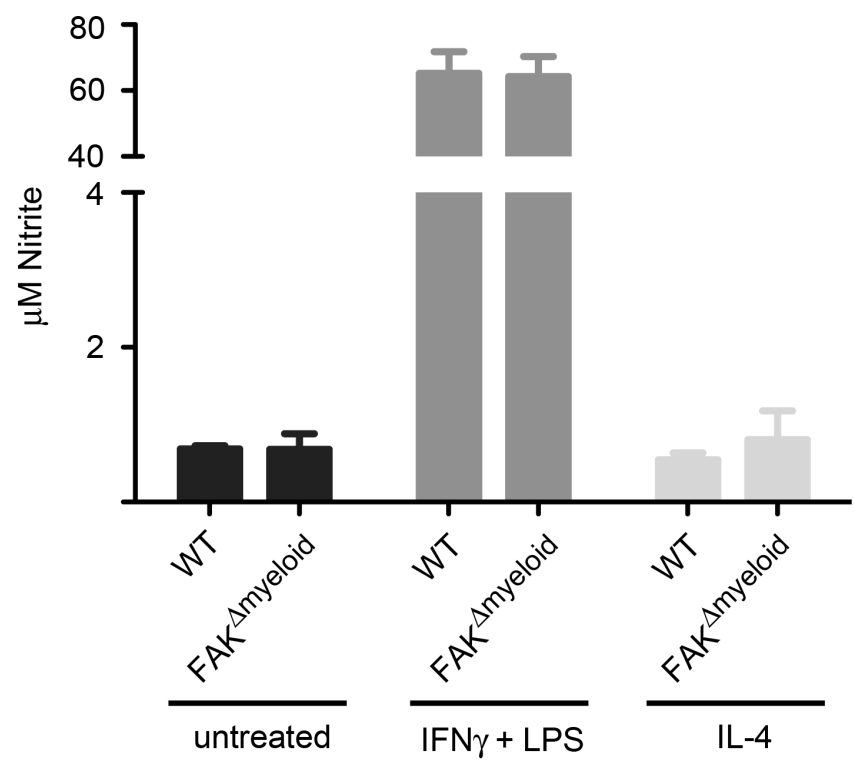


Figure 3.9 FAK does not regulate polarization to M2 activation state in response to IL-4 treatment. (A) BMDM were plated on fibronectin coated coverslips and allowed to adhere overnight. The cultures were washed and treated with media + IL-4 [20ng/ml] for 24 hours, followed by immunofluorsecent staining for the M2 marker CD206 and DAPI (cell nuclei). Representative images of WT and $\mathrm{FAK}^{\Delta \text { myeloid }}$ BMDM in untreated (top panel) and IL-4 (bottom panel) conditions. (B) Immunoblot of cell lysates prepared from WT and FAK ${ }^{\Delta \text { myeloid }}$ BMDM cultured in media alone (lanes 1 and 2) or media + IL-4 [20ng/ml] (lanes 3 and 4) for 24 hours. ERK serves as the loading control. 
A.

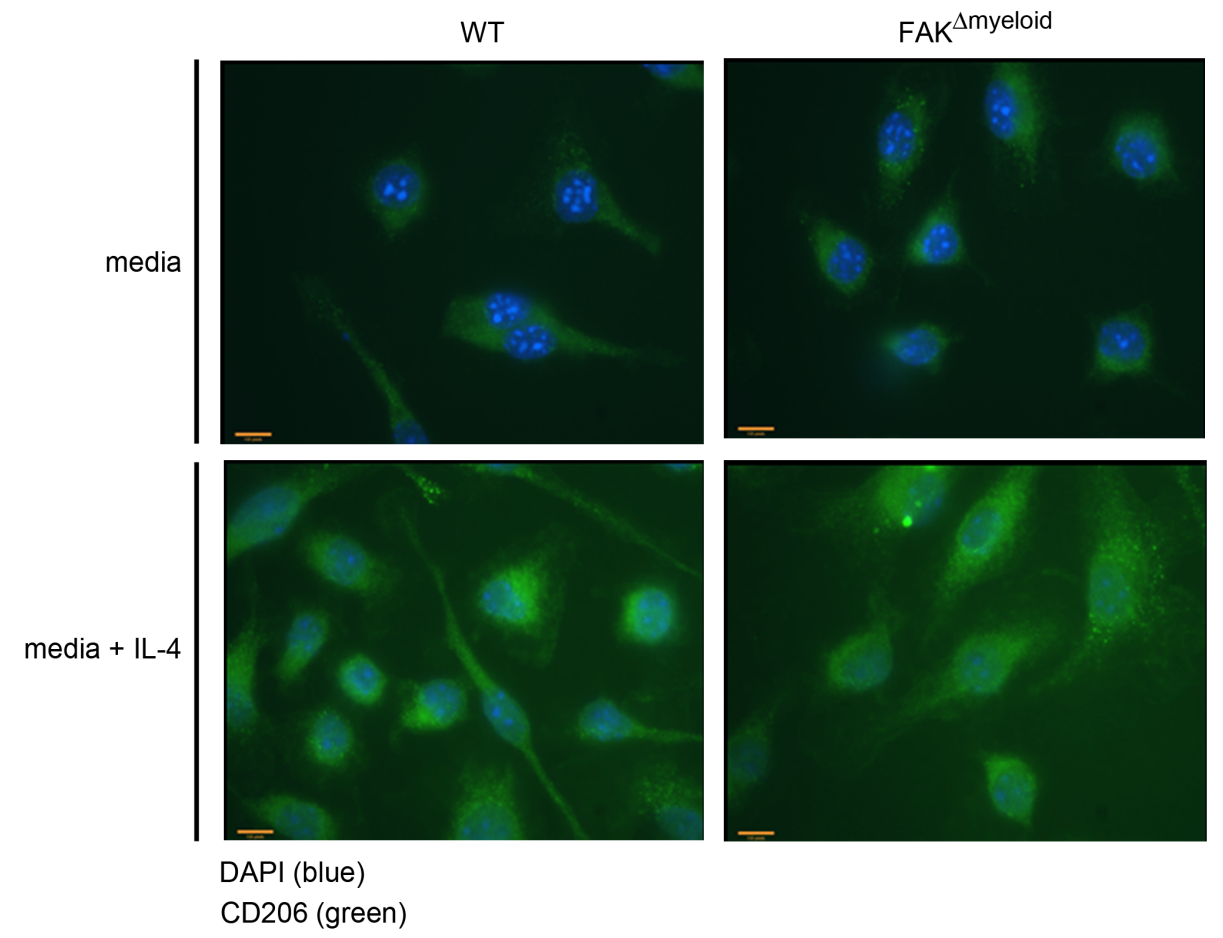

B.

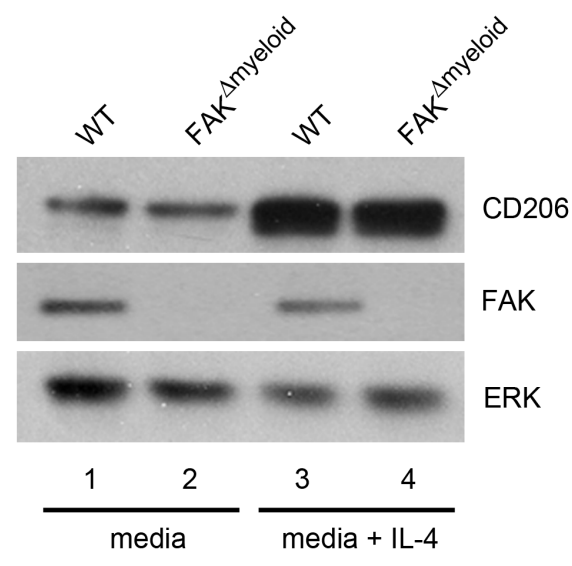


several cell types that FAK regulates the production of MMP-9. Treatment of fibroblasts with TNF- $\alpha$ induced FAK phosphorylation on Y397 and increased secretion of MMP-9 (Mon et al., 2006). Production of MMP-9 in the presence or absence of TNF- $\alpha$ was lost in FAK ${ }^{-/}$cells. In the human ovarian cancer cell line NOM1, FAK regulated the secretion of MMP-9 following treatment with fibronectin (Shibata et al., 1998).

Given that FAK regulates MMP-9 production in numerous cell types and MMP-9 has been shown to be a critical regulatory factor in the tumor microenvironment, we wanted to determine if FAK regulated MMP-9 production in macrophages. MMP-9 can be detected and quantified in the extracellular milieu by zymography. This process involves the resolution of samples harboring MMP on polyacrylamide gels supplemented with gelatin, or denatured collagen. Both the full length inactive and cleaved active forms of the enzyme retain the ability to digest gelatin following this process. Following incubation in reaction buffer and Coomassie stain, the regions on the gel where MMP reside appear clear against the blue stained, undigested gelatin. BMDM from WT and FAK ${ }^{\Delta \text { myeloid }}$ mice were cultured in serum-free conditions for 24 hours, at which time the conditioned media $(\mathrm{CM})$ was collected and concentrated by centrifugal filtration. Cell lysates were generated from the adherent BMDM, resolved on SDS/PAGE, and immunoblotted for proMMP-9, FAK, and ERK. Zymography was performed on the CM samples and quantified by densitometry. All values were normalized to ERK. Our results indicated that there was reduced pro-MMP-9 in the CM from FAK ${ }^{\Delta \text { myeloid }}$ BMDM compared to WT cultures (Figure 3.10A). Conversely, immunoblot revealed that cell-associated pro-MMP-9 was elevated in these cells (Figure 3.10B). These data suggested that FAK regulated secretion of pro-MMP-9 in macrophages. In a previous study conducted in $\mathrm{T}$ lymphoid cells, it was shown that FAK regulated both MMP-9 gene expression and secretion in response to integrin engagement (Segarra et al., 2005). Our data indicate that a similar mechanism may exist in macrophages. 
Figure 3.10 FAK regulates compartmentalization of pro-MMP-9 in BMDM. Adherent BMDM were washed and cultured in serum free media for 24 hours. Conditioned media was concentrated by centrifugal filtration and subjected to gelatin zymography. Cell lysates of adherent cells were prepared for immunoblot analysis. (A) Representative inverse image of zymography gel indicating levels of pro-MMP-9 in the conditioned media of WT (lane 1) and $\mathrm{FAK}^{\Delta \text { myeloid }}$ (lane 2) BMDM. (B) Immunoblot of WT (lane 1) and FAK ${ }^{\Delta \text { myeloid }}$ (lane 2) BMDM cell lysates indicating levels of cell-associated pro-MMP-9. Data are representative of 3 independent experiments. SEM, $*=\mathrm{P}<.05$. 
A.

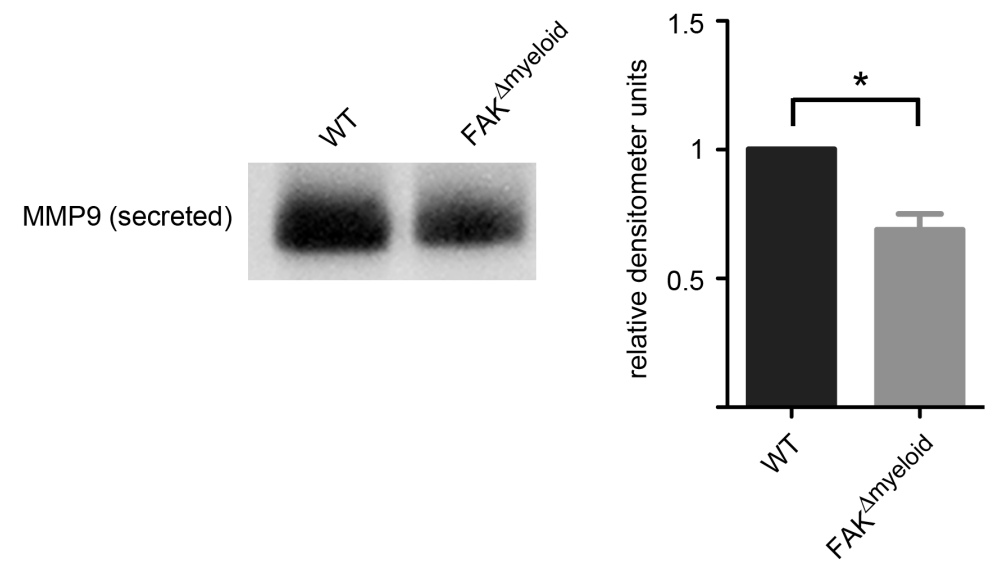

B.

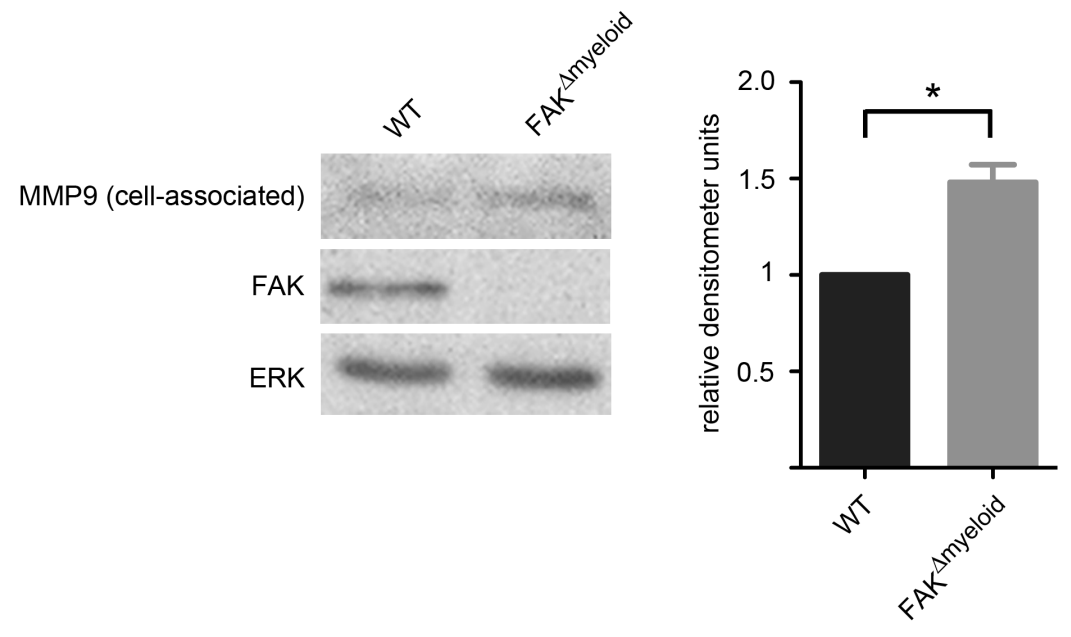




\subsubsection{FAK regulates tumor growth in the MDA-MB-231 orthotopic tumor model}

In parallel with the MMTV-PyVmT tumor study, we also investigated whether FAK in myeloid lineage cells regulated tumor outgrowth in a xenograft model of breast cancer. For this study, we used the MDA-MB-231 human breast cancer cell line, which was derived from the pleural effusion of a 51 year old female cancer patient (Cailleau et al., 1974). This $\mathrm{ER}^{-}$and highly invasive cell line is routinely used in xenograft studies due to its well characterized molecular profile and propensity to form tumors (Price et al., 1990). We implanted $2.0 \times 10^{6}$ MDA-MB-231 cells into the left and right MG4 fat pads and monitored tumor outgrowth. After analyzing numerous mice, $38 \mathrm{WT}$ and $41 \mathrm{FAK}^{\Delta \text { myeloid }}$, it became clear that both mouse strains consistently displayed inefficient tumor development over multiple implant dates (Figure 3.11A). Through personal correspondence with other labs that had used 231 cells in SCID mice, we understood this outcome was not unique. To address this issue, we adapted the protocol described by Jessani et al. in which primary MDA-MB-231 tumors are excised, minced, and cultured ex vivo to increase the numbers of tumor initiating cells (Jessani et al., 2004; Jessani et al., 2005). Consistent with the literature, tumor growth in our study following the orthotopic implantation of this first generation cell line, MDA-MB-231 T1, was dramatically enhanced compared to the parental 231 line (Figure 3.11B). The MDA-MB-231 T1 cells (right panel) were morphologically distinct and had the appearance of mesenchymal cells compared to parental MDA-MB-231 cells (left panel) when grown on fibronectin coated coverslips (Figure 3.11C). Tumor growth as a function of time in $231 \mathrm{~T} 1$ tumors that showed significant growth $\left(>150 \mathrm{~mm}^{3}\right)$ and exhibited exponential growth

(Figure 3.12A). It was determined that tumors in FAK ${ }^{\Delta \text { myeloid }}$ mice had a nearly two fold greater tumor doubling time than tumors grown in WT mice (Figure 3.12B). 
Figure 3.11 Tumorigenesis is enhanced 231T1 cells compared to parental MDA-MB-231 cells. (A) ATCC MDA-MB-231 were implanted into mammary gland \#4 (MG4) of WT and FAK $^{\Delta \text { myeloid }}$ SCID mice and monitored for tumor growth. 38 WT and $41 \mathrm{FAK}^{\Delta \text { myeloid }}$ MG4 were implanted. The number and percentage of MG that displayed no growth, palpable tumors (< $\left.13.5 \mathrm{~mm}^{3}\right)$, growth $\left(13.5 \mathrm{~mm}^{3}-150 \mathrm{~mm}^{3}\right)$, or significant growth $\left(>150 \mathrm{~mm}^{3}\right)$ are indicated. (B) Tumor outgrowth in $63 \mathrm{WT}$ and $63 \mathrm{FAK}^{\Delta \text { myeloid }} \mathrm{MG} 4$ implanted with $231 \mathrm{~T} 1$ cells. (C) Immunofluorescent staining of parental ATCC 231 cells (left panel) and 231T1 cells (right panel) plated on fibronectin. DAPI stain (blue) and Texas red conjugated $\alpha$-phalloidin antibody (red) indicate nuclei and actin filaments, respectively. 
A.

\begin{tabular}{rrrcr} 
& no growth & $\begin{array}{c}\text { palpable } \\
\left(<13.5 \mathrm{~mm}^{3}\right)\end{array}$ & $\begin{array}{c}\text { growth } \\
\left(13.5 \mathrm{~mm}^{3}-150 \mathrm{~mm}^{3}\right)\end{array}$ & $\begin{array}{c}\text { significant growth } \\
\left(>150 \mathrm{~mm}^{3}\right)\end{array}$ \\
\cline { 2 - 5 } WT & $12 / 38(31.6 \%)$ & $26 / 38(68.4 \%)$ & $10 / 38(26.3 \%)$ & $1 / 38(2.6 \%)$ \\
FAK & & & \\
Smyeloid & $27 / 41(65.9 \%)$ & $14 / 41(34.1 \%)$ & $1 / 41(2.4 \%)$ & $0 / 41$
\end{tabular}

B.

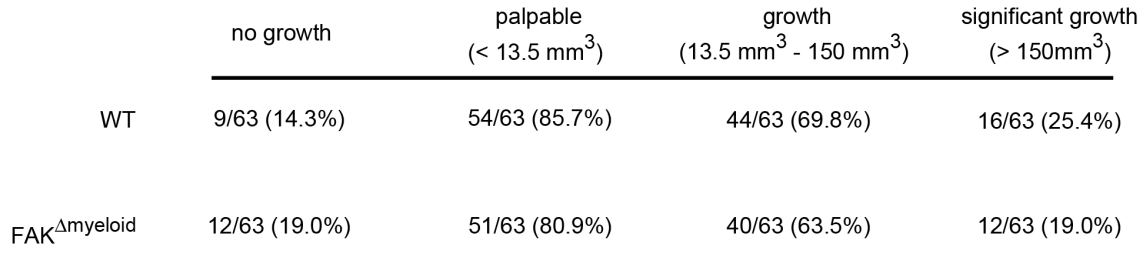

C.
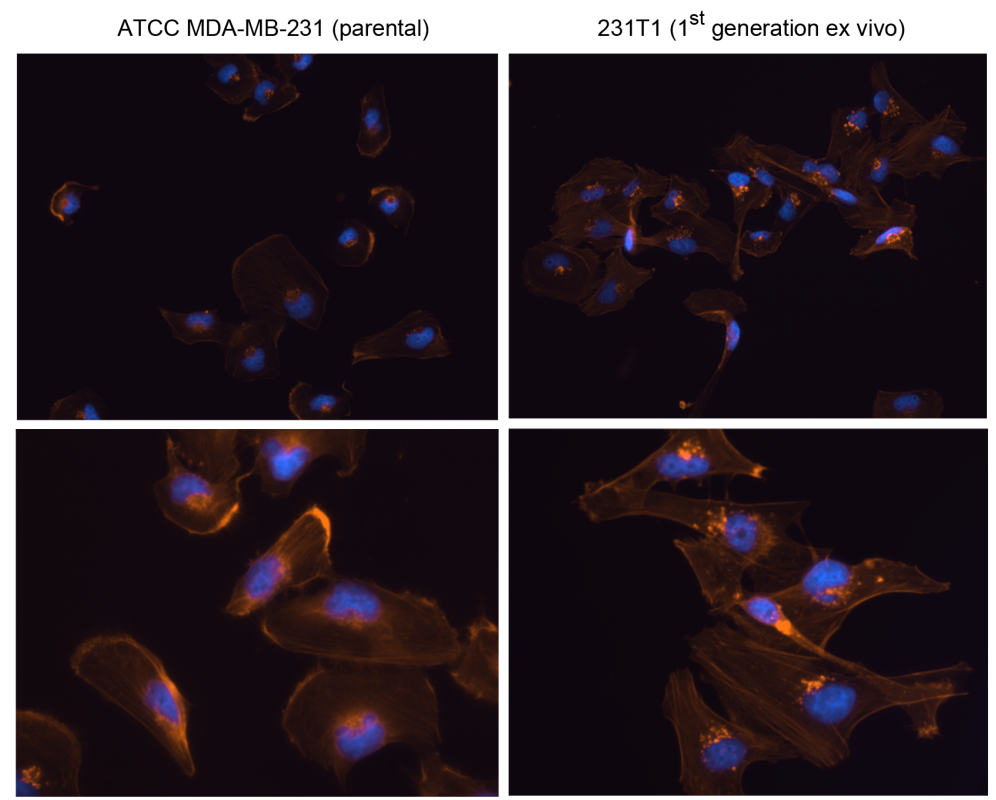

DAPI (blue)

Phalloidin (red) 
Figure 3.12 Tumor doubling time is greater in FAK ${ }^{\text {Amyeloid }}$ mice relative to WT mice. $2.0 \mathrm{x}$ $10^{6} 231 \mathrm{~T} 1$ cells were implanted into MG4 and monitored for growth. (A) Data are representative of all mice from each cohort that displayed significant tumor outgrowth $\left(>150 \mathrm{~mm}^{3}\right)$. (B) The doubling time of tumor growth was determined from the best fit exponential curve applied to each tumor using Prism software. Data points indicate the doubling time for individual tumors. N $=16 \mathrm{WT}, 12 \mathrm{FAK}^{\Delta \text { myeloid }}$. WT black, $\mathrm{FAK}^{\Delta \text { myeloid }}$ blue data points. 
A.

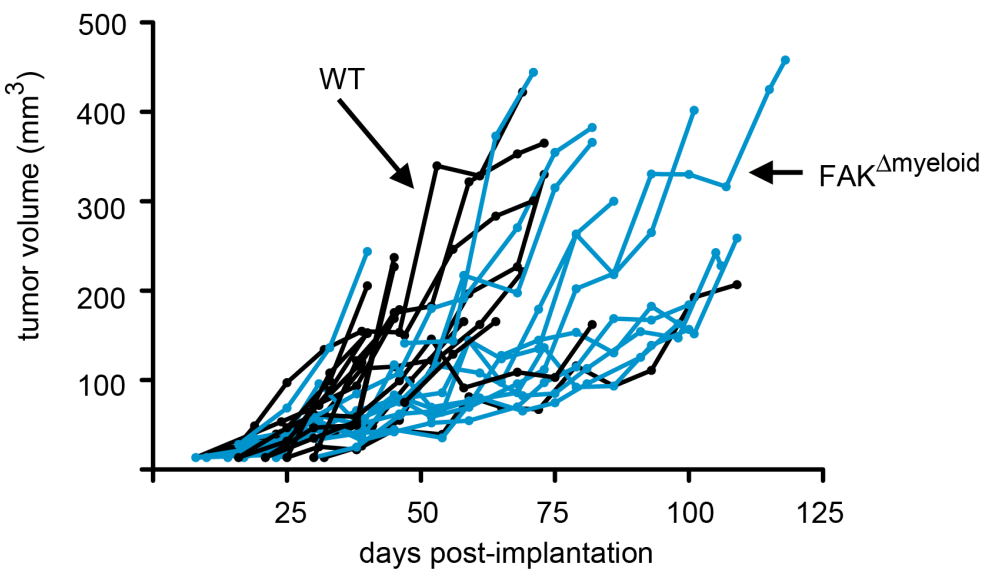

B.

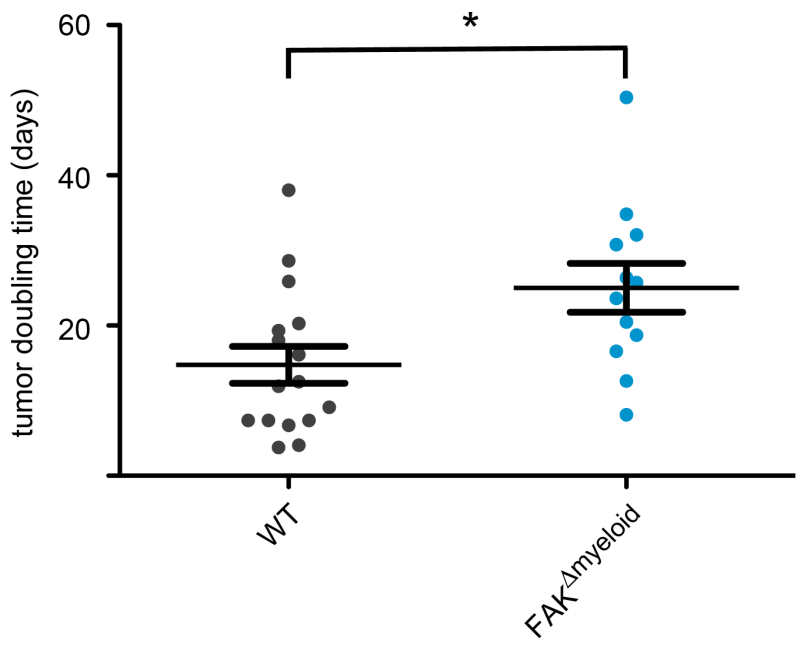




\subsection{Discussion}

The influence of the stromal compartment on tumor progression is well established and is manifested through numerous mechanisms (Tlsty et al., 2005). From a broad perspective, the conditions generated through the combined activities of all stromal elements is at times favorable to tumor growth and development and at times an impediment to this outcome. From a more restricted viewpoint, the cell to cell signaling web that comprises the tumor microenvironment is in constant flux due to changes in cell phenotype over time and movement of cells. The complexity associated with stromal and tumor interactions is amplified when one considers that these occur in a tissue-dependent manner. These associations have been well documented for mononuclear phagocytes on many levels and in many different tumor settings. Tumors consistently display stratified regions delineated by necrosis, vasculature, and extracellular matrix, and it has been shown that macrophage localization in the tumor bed is not uniform (Pollard 2008; Murdoch and Lewis 2005). For example, the tripartite arrangement of cells termed tumor microenvironment of metastasis (TMEM), consisting of an invasive mammary carcinoma cell, a macrophage, and an endothelial cell, has been shown to be a prognostic indicator of systemic metastases in human breast cancer patients (Robinson et al., 2009). In the MMTVPyVmT model, intravital microscopy revealed that macrophage activation state and rate of intratumoral migration are dictated by where the macrophages reside in the tumor (Egeblad et al., 2008). Significant differences existed between the population that localized to the stroma and those cells interspersed alongside carcinoma cells. Numerous examples from immunohistochemical analysis of tumor specimens have substantiated the concept of stromal influence on macrophage phenotype (Lee et al., 2013; Lewis and Pollard 2006).

In this study, we have utilized both in vivo tumor modeling and in vitro cell culture to examine the role of FAK in myeloid lineage cells on tumor progression and macrophage function. Data derived from the MMTV-PyVmT model indicated that early stage primary tumor growth was 
accelerated in mice lacking FAK in myeloid lineage cells. The latency period prior to the appearance of detectable tumor was not influenced by FAK expression in this lineage. Metastasis to the lung, which is a prominent feature of this model, was also not regulated by FAK in these cells. To provide insight into what could be contributing to the observed differences in tumor size, we examined characteristics commonly attributed to tumor associated macrophages that impact tumor growth and invasive potential. FAK did not regulate the ability of BMDM to polarize to either an M1 or M2 activation state in response to IFN- $\gamma+$ LPS or IL-4, respectively. The ability of tumors to form vessels appeared to be a FAK-independent process as well. MMP-9 compartmentalization in macrophages, however, was regulated by FAK. Our data showed that the loss of FAK led to less pro-MMP-9 in the extracellular milieu but enhanced levels associated with the cells. This suggested that FAK modulated the secretion of pro-MMP-9.

An inverse tumor outgrowth phenotype was observed in the orthotopic xenograft MDA-MB-231 tumor model. In the cohort of mice that displayed significant growth $\left(>150 \mathrm{~mm}^{3}\right)$, the doubling time was significantly greater in $\mathrm{FAK}^{\Delta \text { myeloid }}$ mice, meaning that it took longer for tumors in these mice to double in size. It has yet to be determined if differences in TAM activation state exist in the tumor microenvironment.

The systems utilized in our studies varied significantly and thus must be thoroughly considered in our analysis. In the MMTV-PyVmT model, transformation and hyperplasia occurred de novo due to expression of a potent oncogene, tumors progressed through defined stages, and metastases formed in the lung due to conditions in the mammary epithelium that promoted invasion. Prior studies conducted in mice that have a near complete ablation of macrophages due to the deletion of M-CSF (op/op mice) indicated that macrophages play a prominent role primarily in regulating the angiogenic switch required for progression to carcinoma and interacting with tumor cells and promoting their migration during invasion (Lin et al., 2001; Lin et al., 2006). FAK does not appear to be involved in these late stage events directed by macrophages, as metastasis and vessel 
density were similar in WT and FAK ${ }^{\Delta \text { myeloid }}$ mice. However, the absence of FAK resulted in enhanced tumor growth during the early stages of progression, a result very different to what was observed in the op/op mouse where complete macrophage loss had no impact on early stage growth. This suggested to us that FAK played an active role in macrophage functions that either blocked pro-tumor activities and/or promoted anti-tumor activities. Future studies will be needed to determine the mechanism(s) orchestrating the phenotype we observed, but there are several possibilities that should be considered at this time. DeNardo et al. made a very convincing argument that macrophage activation state in the later stages of progression was critical to the invasion and metastatic process (DeNardo et al., 2009). They illustrated that secretion of IL-4 by tumor-infiltrating $\mathrm{CD} 4^{+} \mathrm{Th} 2$ cells polarized macrophages to the tumor-promoting M2 activation state. The polarized macrophages in turn regulated the invasive behavior of the carcinoma cells, due in part to macrophage secretion of EGF. They also found that TAM in CD4-deficient mice expressed enhanced levels of cytokines associated with an M1 activation state and reduced M2 cytokines. Our results placed in context with these data suggest that FAK may function to block the early stage accumulation of $\mathrm{CD} 4{ }^{+} \mathrm{Th} 2$ cells in the tumor microenvironment. With the loss of FAK, enhanced levels of Th2 cells may increase M2-associated factors in the milieu that drive tumor growth. Notably, the levels of VEGF-A, a potent angiogenic factor, were independent of $\mathrm{CD}^{+} \mathrm{T}$ cell accumulation, and thus could help explain normal vessel density in $\mathrm{FAK}^{\Delta \text { myeloid }}$ mice. However, this model would not account for the equivalent levels of metastatic growth observed in the $\mathrm{FAK}^{\Delta \text { myeloid }}$ mice relative to WT mice. If loss of FAK led to more Th2 cells and more IL-4, we would have expected enhanced metastatic burden in these mice. Our in vitro data indicated that IL-4 mediated-macrophage polarization was FAK-independent. This could, however, highlight a second role for FAK in the tumor microenvironment. We have shown previously that FAK regulates macrophage migration to soluble factors commonly associated with the tumor microenvironment, including M-CSF. In our model, perhaps the skew toward a more invasive phenotype due to enhanced IL-4 signaling in $\mathrm{FAK}^{\Delta \text { myeloid }}$ mice would be balanced by a reduced 
capacity of FAK ${ }^{\Delta \text { myeloid }}$ TAM to mediate tumor cell invasion. These are all speculative scenarios, given that we do not know if the proportion, activation state, and/or localization of mononuclear phagocytes in tumors from $\mathrm{FAK}^{\Delta \text { myeloid }}$ mice are altered relative to WT tumors. Additional studies will need to address these topics, in addition to the number and phenotype of adaptive immune cells in the tumor microenvironment as a function of FAK expression in myeloid lineage cells.

Our results from the SCID model suggest that FAK regulates tumor associated myeloid cell function independent of adaptive immune influence. It is difficult to make comparisons across the two models given the differences in tumor cells involved, the methods utilized to induce tumor formation, and the parameters of tumor progression. The data in this model are very speculative as well, as we currently do not know the proportion or phenotype of mononuclear phagocyte populations at the tumor site. We could speculate that the interaction of mononuclear phagocytes with $231 \mathrm{~T} 1$ cells in the tumor may induce an M2 state, and that FAK modulates this response. This would result in reduced production of pro-tumor factors in $\mathrm{FAK}^{\Delta \text { myeloid }}$ tumors relative to WT tumors, leading to a reduced rate of tumor growth in these mice. Future studies will need to address this, as it has been established that MDA-MB-231 cells secrete significant levels of the differentiating factors M-CSF and GM-CSF (Pederson et al., 1999). Additionally, the development or acquisition of metastatic MDA-MB-231 variants could be very helpful in determining if FAK plays a role in macrophage processes responsible for tumor cell invasion. 


\section{Chapter 4}

Future Directions

\subsection{Introduction}

The goals of my dissertation research were to characterize the regulated expression of FAK and Pyk2 in myeloid lineage cell populations, determine if monocyte differentiation is influenced by FAK and Pyk2, and utilize murine cancer models to ascertain if FAK regulates macrophage functions critical for progression of murine breast carcinomas. Our results indicate that FAK and Pyk2 are differentially regulated during monocyte differentiation and under specific conditions control this process. We also found that tumor growth is regulated by FAK expression in myeloid lineage cells. Given the characteristics of heterogeneity and functional plasticity associated with mononuclear phagocytes, the direction of future study in the area of FAK and Pyk2 regulation of myeloid cell function should incorporate approaches to better characterize FAK and Pyk2 expression in different myeloid populations and test the functional properties of specific cell populations.

\subsection{What mononuclear phagocyte populations express FAK and Pyk2?}

The literature indicates that FAK and Pyk2 protein expression are not uniform in myeloid lineage cells. FAK was detected in progenitor cells from the bone marrow, cell populations that were derived from the in vitro treatment of $\mathrm{BM}$ with differentiation factors, and $\mathrm{CD} 11 \mathrm{~b}^{+}$cells from the peritoneum under steady state and inflammatory conditions (Glodek et al., 2007; Kume et al., 1997; Owen et al., 2007). Pyk2 has also been detected in mature macrophages and less differentiated cells (Okigaki et al., 2003). The work presented in this thesis has expanded our 
understanding of FAK and Pyk2 expression in this lineage by showing that FAK increases during differentiation in a process that tracks with the loss of Ly6C. Pyk2 expression correlates with differentiation as well, although our data show that it is expressed earlier than FAK and concomitant with F4/80 upregulation.

These studies raise the question of why FAK and Pyk2 are so tightly regulated in different cell populations. Differentiation of myeloid lineage cells in the bone marrow occurs in a series of tightly regulated stages that results in increasingly more committed lineage related cell populations (Figure 1.2). The surface antigens that distinguish these populations are well characterized and permit isolation of cells, yet this methodology has not yet been applied to the study of FAK and Pyk2. Moreover, it is unknown if the proportion of these populations in the bone marrow are altered in $\mathrm{FAK}^{\Delta \text { myeloid }}$ and $\mathrm{Pyk}^{-/-}$mice. As illustrated in Figures 1.3 and 1.4, committed monocytes express other well characterized markers that could be exploited for selective purposes. We focused on Ly6C and F4/80 in our studies, as they are prominent markers of differentiation, yet other molecular markers shown in these figures provide the opportunity to measure FAK and Pyk2 expression in cell populations that have unique distinguishing and functional qualities. At this time, it is not known if any correlation exists between FAK and Pyk2 with these markers. Given the heterogeneity of mononuclear phagocyte function associated with these various populations, future experiments on defined subsets of cells should be regularly accompanied with parallel analyses of the expression profile of FAK and Pyk2. Isolation of these populations by FACS based on established surface antigen profiles would permit comparative analyses at both the genetic and protein levels. Not only would FAK and Pyk2 expression be characterized in these populations, but we would learn about other proteins that might be regulated coincidentally with these molecules. Finally, this approach could be applied to a tumor setting, where heterogeneity of myeloid cell populations is commonplace. Differentiation and functional plasticity contribute to monocyte heterogeneity in the tumor microenvironment, yet we 
have not determined FAK and Pyk2 expression in, or their regulation of, these populations in situ. Isolation of distinct populations based on surface antigen in a hypothesis-driven approach would allow one to not only test the biochemical properties of these cells, but utilize them in controlled ex vivo assays.

We have utilized flow cytometry to track monocytes populations during differentiation. The commercially available FAK antibodies have been tested extensively by our lab and are not applicable in this format due to a high degree of non-specific antibody binding. An alternative approach to the methodology described above would be to develop a FAK (or Pyk2) reporter mouse, in a similar vein to the $\mathrm{CX} 3 \mathrm{CR} 1^{\mathrm{GFP} /+}$ mouse, which would permit determination of FAK (or Pyk2) expression in mononuclear phagocytes utilizing fluorescence as a surrogate marker. Given that FAK and Pyk2 are significantly upregulated with the loss and Ly6C and gain in F4/80, respectively, clear distinctions between monocyte populations by fluorescence intensity are likely achievable. This approach could be beneficial in the elucidation of when FAK and Pyk2 are turned on during myelopoiesis, as well as observing the differentiation of monocyte populations in vivo. One burning unanswered question is at what point is differentiation initiated in the transendothelial/subendothelial migration of $\mathrm{Ly} 6 \mathrm{C}^{+}$monocytes at peripheral sites, and relatedly, at what point does FAK get upregulated? The adoptive transfer of Ly6C ${ }^{\text {high }}$ monocytes (which express minimal detectable $\mathrm{FAK}$ ) from $\mathrm{FAK}^{\mathrm{GFP} /+}$ mice and subsequent analysis of GFP expression would address this. Of particular interest would be histology on tissue samples that captured monocytes in circulation, adherent to the endothelial wall, and within the tissue stroma. 


\subsection{How does FAK coordinate with other molecules that regulate differentiation from Ly6C $^{\text {high }}$ to Ly6C ${ }^{\text {low }}$ monocytes?}

Through FACS and immunomagnetic column separation, we were able to segregate monocyte populations showing high or low Ly6C expression under various conditions in vitro and in vivo. In all populations analyzed, increased FAK expression correlated with the loss of Ly6C. Under conditions that showed the greatest level of distinction between $L y 6 C^{\text {high }}$ and Ly6C $\mathrm{C}^{\text {low }}$ populations, FAK was essentially undetectable in the Ly6 $\mathrm{C}^{\text {high }}$ monocyte population. These data indicate that FAK is expressed only in the later stages of monocyte differentiation. We also showed that FAK was not absolutely required for bone marrow progenitor cells to differentiate into terminally differentiated macrophages if given adequate time and an appropriate stimulus. In vitro, this requires approximately four days under M-CSF conditions. At earlier times (two days), we saw a modest but significant decrease in the proportion of differentiated $\mathrm{Ly}^{-} \mathrm{C}^{-} \mathrm{F} 4 / 80^{+}$ monocytes in the absence of FAK (Figure 2.9D). Combined with our results showing that FAK expression is initiated during the differentiation of $\mathrm{Ly} 6 \mathrm{C}^{+} \mathrm{F} 4 / 80^{+}$monocytes to $\mathrm{Ly} 6 \mathrm{C}^{-} \mathrm{F} 4 / 80^{+}$ monocytes (Figure 2.8C), we concluded that FAK functions to stimulate monocyte differentiation during the later stages of this process, but that it was not absolutely required for this process.

In addition, given the rapid kinetics of monocyte differentiation during M-CSF treatment, it would be advantageous to study FAK-regulated aspects of late stage monocyte differentiation under conditions in which the transition between $\mathrm{Ly} 6 \mathrm{C}^{+} \mathrm{F} 4 / 80^{+}$and $\mathrm{Ly} 6 \mathrm{C}^{-} \mathrm{F} 4 / 80^{+}$cells is slow. Under these conditions, it might be possible to better track the accumulation of Ly6C $\mathrm{F}^{-} / 80^{+}$cells over time. Alternatively, monocyte populations at different stages of differentiation and distinguished by relative levels of FAK and Pyk2 could be isolated by FACS, cultured under various conditions that drive differentiation, and analyzed by flow cytometry for differentiation markers. This would allow a more direct examination of the ability of one population to differentiate into another in the presence or absence of FAK and/or Pyk2. It would also provide 
insight into how different monocyte populations respond to stimuli as a function of FAK/Pyk2 expression. For example, our data indicate that the proportion of $\mathrm{Ly} 6 \mathrm{C}^{+} \mathrm{F} 4 / 80^{+}$monocytes is reduced with the loss of Pyk2. It is not known if this population has functional significance, and if so, whether the function of Pyk2 is regulated at this stage. This could be addressed through ex vivo studies of isolated $\mathrm{Ly} 6 \mathrm{C}^{+} \mathrm{F} 4 / 80^{+}$monocytes.

A second factor to take into account is that our in vitro studies were performed on untreated polystyrene plastic. The literature on matrix regulation of monocyte differentiation is limited, but there is some evidence that suggests that factors such as fibronectin and collagen influence monocyte differentiation (Kamoshida et al., 2012; Jacob et al., 2002; Jacob et al., 2001; Sudhakaran et al., 2007; Kaplan and Gaudernack, 1982). Given the prominent role of FAK as a regulator of signaling through integrins, future in vitro studies should also be conducted on various extracellular matrix components. These conditions may enhance integrin engagement and FAK activation, and thus illuminate FAK'dependent events during differentiation. The results from these studies could be particularly applicable to tissue settings that have high stromal content. An example would be in tumors, where myeloid lineage cells have been shown to localize in stromal regions.

\subsection{What is the role of FAK and Pyk2 in the effector function of mononuclear phagocyte populations?}

Our data indicate that the only committed monocytic cells that express significant levels of FAK are those in the late stages of differentiation. These cells are characterized by low levels of Ly6C and high F4/80 expression. This indicates that any function of FAK in committed monocytes will be restricted to highly differentiated Ly6C $\mathrm{C}^{-}$cells. Pyk2 is expressed during earlier stages of differentiation than is FAK, and persists in more differentiated cells. Given that it is expressed in 
Ly6 $\mathrm{C}^{\text {high }}$ and $\mathrm{Ly} 6 \mathrm{C}^{\text {low }}$ populations from in vitro-derived and tissue monocytes, Pyk2 should be considered as having the potential to influence both differentiation and function of all monocyte populations.

What then are the possible implications of this dichotomy in relation to mononuclear phagocyte function? Previous studies have shown that both FAK and Pyk2 regulate migration of mature macrophages in vitro (Owen et al., 2007; Okigaki et al., 2003). Myeloid lineage cells that are depleted of FAK and Pyk2 also display reduced accumulation at sites of inflammation in vivo relative to WT cells. However, it is not clear if this defect is due to altered migration into the inflammatory site or if another mechanism could account for the reduced proportion of these cells. Our data would argue that the migration of $\mathrm{Ly}^{+} \mathrm{C}^{+}$monocytes into tissues could be regulated by Pyk2, but not by FAK. It is established that under inflammatory conditions, Ly6C ${ }^{+}$ monocytes are mobilized in the bone marrow and account for the vast majority of cells that ultimately extravasate from the circulation into tissues (Kamei et al., 2010; Shi et al., 2011). These cells have to cross two endothelial cell barriers for this to occur. Our experiments, which were conducted at steady state, indicated a very modest decrease in the proportion of Ly $6 \mathrm{C}^{+} \mathrm{F} 4 / 80^{+}$monocytes in the blood of Pyk $2^{-/-}$mice. While we concluded that this was due to Pyk2 -regulated enhanced differentiation of this population, we could not rule out the possibility that it was due to altered transendothelial migration of these cells from the bone marrow. A complete analysis of $\mathrm{Ly}_{6 \mathrm{C}} \mathrm{F}^{+} / 80^{-}, \mathrm{Ly} 6 \mathrm{C}^{+} \mathrm{F} 4 / 80^{+}$, and $\mathrm{LyC}-\mathrm{F} 4 / 80^{+}$monocyte populations in the $\mathrm{BM}$, blood, and tissue of Pyk $2^{-/-}$mice under inflammatory conditions, which would stimulate the production of $\mathrm{Ly}_{6} \mathrm{C}^{+}$monocytes and their trafficking, would help to resolve this issue by identifying potential Pyk2-dependent steps in Ly6 $\mathrm{C}^{+}$trafficking in vivo.

Conversely, FAK regulation of monoctye trafficking is likely limited to Ly6C $\mathrm{C}^{\text {low }}$ mononuclear phagocytes since these are the only cells that express FAK. Evidence indicates that the Ly6C $\mathrm{C}^{\text {low }}$ monocyte population does not emigrate from the $\mathrm{BM}$ and is instead derived from the 
differentiation of Ly6C $\mathrm{C}^{\text {high }}$ blood monocytes (Yona et al., 2013). Emigration of this population from the blood into tissues is thought to be very minimal during steady state compared to Ly6 $\mathrm{C}^{+}$ monocytes. Instead, Ly6 $\mathrm{C}^{\text {low }}$ cells in the blood have acquired significant attention due to their apparent function as patrollers of the vascular endothelium in peripheral tissues, which can then result in the induction of rapid transendothelial migration into tissues during inflammation (see Chapter 1 and Auffray et al., 2007). We showed in vitro that Ly6C $\mathrm{C}^{\text {low }}$ monocytes lacking FAK display reduced migration toward M-CSF. This defect was not observed in Ly6C ${ }^{\text {high }}$ monocytes, indicating that FAK may play an important role in regulating Ly6 $\mathrm{C}^{\text {low }}$ monocyte migration in vivo. We suggest that the migratory defect exhibited by $\mathrm{FAK}^{-/}$Ly $6 \mathrm{C}^{\text {low }}$ monocytes in vitro may be even more pronounced under inflammatory conditions that require the rapid emigration of Ly6 $\mathrm{C}^{\text {low }}$ monocytes into tissues. This could be measured by adoptively transferring fluorescently labeled monocytes from WT and $\mathrm{FAK}^{\Delta \text { myeloid }}$ mice into mice under inflammatory conditions followed by tissue analysis for infiltrated Ly6C $\mathrm{C}^{\text {low }}$ cells. The same methodology could be applied toLy6 $\mathrm{C}^{\text {high }}$ and $\mathrm{Ly} 6 \mathrm{C}^{\text {low }}$ monocytes from $\mathrm{Pyk} 2^{-/-}$mice.

Recent evidence indicates that monocyte differentiation has evolved to maintain both the Ly6 $\mathrm{C}^{\text {high }}$ and the Ly6C $\mathrm{C}^{\text {low }}$ populations (Ingersoll et al., 2010). The transcription factor NR4A1 (Nur77), a member of the NR4A subfamily of orphan nuclear receptors, has received significant interest recently as it appears to be required for the generation of Ly6C' monocytes (Hanna et al., 2012). It was shown that $\mathrm{Nr} 4 a 1$ mRNA was expressed at significantly higher levels in BM Ly6C monocytes compared to $\mathrm{Ly} 6 \mathrm{C}^{+}$, MDP, and CMP populations. Protein levels were also higher in Ly6 $\mathrm{C}^{-}$monocytes relative to the Ly $6 \mathrm{C}^{+}$cohort. $\mathrm{Nr} 4 a 1^{--}$mice were found to harbor significantly lower levels of Ly6C $\mathrm{C}^{-}$monocytes in the $\mathrm{BM}$, blood, and spleen relative to WT mice, while the number of Ly6 $\mathrm{C}^{+}$monocytes was unchanged in these tissues. These data indicated that NR4A1 was a critical regulator of monocyte differentiation. A study published soon thereafter showed that deletion of NR4A1 resulted in enhanced M1 polarization in macrophages. The critical 
regulatory role of NR4A1in monocyte differentiation and effector function, combined with a clear distinction between NR4A 1 expression in Ly6 $\mathrm{C}^{\text {high }}$ and Ly6 $\mathrm{C}^{\text {low }}$ monocytes, suggest that consideration should be given to include it as a marker of Ly6C ${ }^{\text {low }}$ monocytes. The literature indicates that this is starting to occur (Carlin et al., 2013).

The similar expression profiles between FAK and NR4A1 as a function of differentiation state raise the possibility that a relationship might exist between FAK and NR4A1, and that FAK may also serve as an identification marker of Ly6 $\mathrm{C}^{\text {low }}$ monocytes. However, our studies would suggest that the loss of FAK would appear to be less impactful than is the loss of NR4A1. It is not currently known whether there is any biochemical relationship between NR4A1, FAK, and/or Pyk2, or if expression of one species is contingent upon the presence of the others. One possibility is that NR4A1 expression or activity is regulated by FAK in addition to other factors. NR4A1 has been shown to function as an immediate early gene in other cell types given its rapid induction in response to growth factors and cytokines (Martinez-Gonzalez et al., 2005). A second possibility is that FAK and/or Pyk2 may be involved in the regulation of NR4A1 activity. Evidence indicates that transcriptional activity of members of the NR4A subfamily is not regulated by ligand binding but instead by posttranslational modification. For example, phosphorylation events induced by the ligand-mediated binding of cell surface receptors can negatively and positively regulate NR4A1 expression and activity. In one possible scenario, FAK and/or Pyk2 may regulate NR4A1 function through activation of Akt, which could then phosphorylate Ser-350 on NR4A1, inhibit its DNA binding capabilities, and negatively regulate genes that might otherwise block differentiation or control effector functions. Analysis of NR4A1 expression and activity in isolated monocyte populations that have established FAK and Pyk2 expression profiles would provide insight into their relationship with one another.

NR4A1 ${ }^{+}$Ly $6 \mathrm{C}^{\text {low }}$ monocytes display distinctive migratory behavior on vascular endothelium, leading to their description as patrolling monocytes, and respond to inflammatory cues that 
trigger rapid extravasation. A role for FAK in transendothelial migration of Ly6 $\mathrm{C}^{\text {low }}$ cells toward an inflammatory signal could help to explain our results from the MMTV-PyVmT tumor study. Our working model is that FAK expression promotes anti-tumor and/or blocks pro-tumor functions of mononuclear phagocytes during the early stages of tumor outgrowth. One hypothesis would be that FAK is required for the patrolling behavior of $\mathrm{Ly} 6 \mathrm{C}^{-}$monocytes. It has been shown that activation of patrolling $\mathrm{Ly} 6 \mathrm{C}^{-}$monocytes through toll-like receptor 7 (TLR7) ligation increases dwell time on the endothelium, resulting in the accumulation of neutrophils to the site and killing of endothelial cells by the neutrophils (Carlin et al., 2013). The Ly6C ${ }^{-}$monocytes then function to remove the cellular debris from the area. Although it is speculative, this process may occur in the tumor microenvironment and help to inhibit tumor growth. One possibility is that an anti-tumor response is mediated through the enhanced accumulation of neutrophils at the site. Interestingly, we have found by Luminex that more granulocyte colony stimulating factor (G$\mathrm{CSF}$ ) is associated with tumors in WT mice compared to those in $\mathrm{FAK}^{\Delta \text { myeloid }}$ mice at day 105 (data not shown). G-CSF promotes the proliferation and differentiation of neutrophils (Hamilton, 2008). We have not yet determined, however, if neutrophils are more prevalent in WT tumors. An alternative hypothesis would be that the increased presence of $\mathrm{Ly} 6 \mathrm{C}^{-}$monocytes on the vascular endothelium in tumors potentiates tumor growth. Under steady state, the dwell time of Ly6C ${ }^{-}$ monocytes prior to release was reported to be 9 min., which was increased to $25 \mathrm{~min}$. following TLR7 activation (Carlin et al., 2013). Preliminary evidence from the study indicated that this process entailed specificity, as activation with LPS did not induce a similar response in the monocytes. Perhaps in the tumor, enhanced accumulation of $\mathrm{Ly}^{-} \mathrm{C}^{-}$monocytes is exacerbated when FAK is absent in these cells. Given that FAK regulates cytoskeletal rearrangements critical for tail retraction, it is possible that the loss of FAK would increase dwell time once the Ly6 $\mathrm{C}^{-}$ monocytes become adherent. This in turn could cause increased neutrophil recruitment, excessive necrosis, and increased inflammation which, in several instances, has been shown to promote tumor growth (Elinav et al., 2013). 
While work that showed macrophages regulate the angiogenic switch and metastasis in this model emphasized the capabilities of macrophages, it also shifted the focus to later stage events, as macrophage ablation has very modest impact on primary growth. Our results demonstrate that FAK in mononuclear phagocytes plays a role to keep tumors smaller, however its regulatory function remains elusive given that this phase is comparatively less well studied and consequently the mechanisms driving growth are not well delineated. Potential mechanisms are illustrated in Figure 4.1 and incorporate what is known about the interactions of the MPS with cellular and non-cellular factors in the tumor microenvironment. FAK may be necessary to drive differentiation of M-MDSC to TAM in response to tumor associated factors (1). Results from our work showed that differentiation of $\mathrm{Ly} 6 \mathrm{C}^{+} \mathrm{F} 4 / 80^{+}$to $\mathrm{Ly} 6 \mathrm{C}^{-} \mathrm{F} 4 / 80^{+}$monocytes was modestly reduced in response to M-CSF in monocyte cultures from $\mathrm{FAK}^{\Delta \text { myeloid }}$ mice (Chapter 2). A similar process may occur in vivo, and could potentially be magnified due to the heterogeneity of tumorassociated stimuli. It is not known, however, if increased accumulation of M-MDSC would result in enhanced tumor formation. Studies underway are aimed to characterize monocyte populations, both phenotypically and functionally, that comprise the primary tumor mass in WT and $\mathrm{FAK}^{\Delta \text { myeloid }}$ tumors. As addressed earlier, FAK may also regulate the migration of monocytes that have lost Ly6C expression, which could manifest at several stages of trafficking, including initial adhesion (2), migration along the endothelium (3), release from the endothelium (4), and transmigration across the endothelium (5). Earlier reports have indicated that this is a CX3CR1 and LFA-1 regulated process, and there is literature indicating that loss FAK may impede signaling mediated through CX3CR1 (Fujita et al., 2012). Data from Carlin et al. implicated signaling through TLR7 as a critical and specific regulator of Ly6C function, however it remains to be determined if FAK regulates signaling through TLR7 in monocytes. In conjunction with this is the ability of tumor associated mononuclear phagocytes to migrate within the tumor bed and regulate the localized production of soluble factors (6). Our data that showed reduced secreted 
Figure 4.1 Potential FAK-dependent mechanisms utilized by tumor associated mononuclear phagocytes to regulate primary tumor outgrowth. The schematic illustrates yet to be determined FAK-regulated monocyte/macrophage processes or functions in the tumor microenvironment, including (1) M-MDSC differentiation to TAM, (2) adherence to endothelial cells, (3) migration along the endothelium, (4) release from the endothelium, (5) transmigration, (6) secretion of soluble factors, and (7) recruitment/blocked recruitment of adaptive immune cells. All of these activities could be involved in regulating the association of Ly6C ${ }^{\text {high }}$, Ly $6 C^{\text {low }}$, and adaptive immune cells (8). 


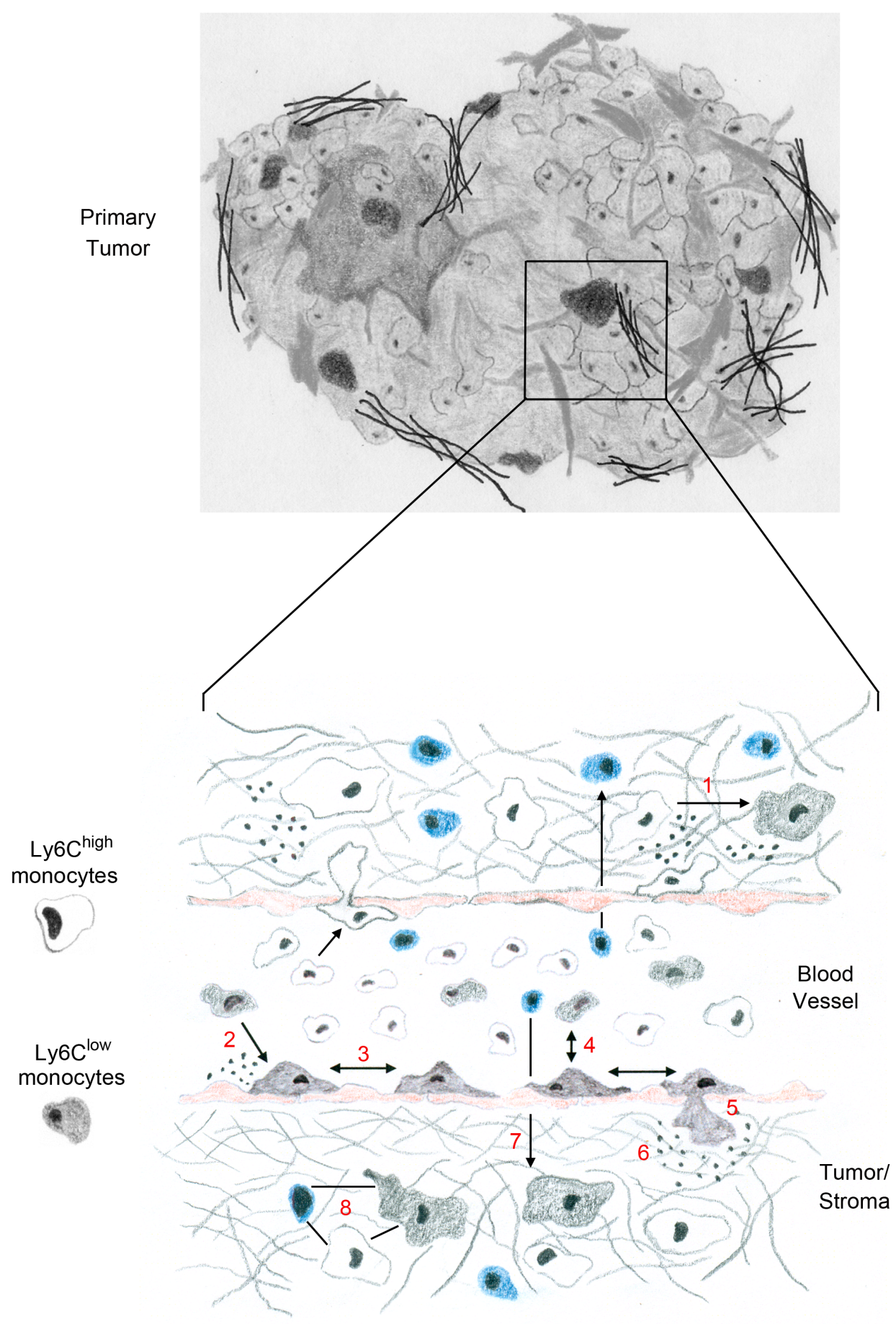


pro-MMP9 in BMDM with the loss of FAK suggest that FAK may regulate the production of other mediators (Chapter 3).

Interestingly, conditional knockout of VEGF-A in myeloid lineage cells resulted in enhanced primary tumor progression and larger primary tumors (Stockmann et al., 2008). A potentially significant yet unexplored area of research is FAK-regulated accumulation and function of adaptive immune cells (7). Perhaps FAK in mononuclear phagocytes is involved in recruitment of cytotoxic $\mathrm{T}$ cells or NK cells, or the inhibition of $\mathrm{T}$ regulatory cell accumulation. This area is unexplored, but determination of tumor infiltrating lymphocyte populations in WT and $\mathrm{FAK}^{\Delta \text { myeloid }}$ mice would be a first step. It would also lead to a more refined picture of how Ly6C ${ }^{\text {high }}$, Ly6C ${ }^{\text {low }}$, and adaptive immune cells associate (8). All future analyses must bear in mind that it is the accumulative effect of FAK-regulated MPS function that is responsible for our tumor phenotype, and this could potentially involve a deregulated balance of both pro and anti-tumor mononuclear phagocyte activities.

To conduct future experiments that involve monocyte trafficking and analyses of Ly6 $\mathrm{C}^{\text {low }}$ monocyte behavior, it would be beneficial to be able to observe Ly6 $\mathrm{C}^{\text {low }}$ monocytes on the vasculature. This has been achieved in the past through the use of $\mathrm{CX} 3 \mathrm{CR} 1^{\mathrm{GFP} /+}$ mice. As indicated in Chapter 1 , Ly6C $\mathrm{C}^{\text {high }}$ and $\mathrm{Ly} 6 \mathrm{C}^{\text {low }}$ monocyte populations in this model can be distinguished by GFP intensity (Auffray et al., 2007). Ly6C ${ }^{\text {low }}$ monocytes, which express relatively high levels of $\mathrm{CX} 3 \mathrm{CR}$, are highlighted on the endothelium by bright green fluorescence. Therefore, crossing the WT and $\mathrm{FAK}^{\Delta \text { myeloid }}$ mice onto a $\mathrm{CX} 3 \mathrm{CR} 1^{\mathrm{GFP} /+}$ strain would allow one to observe Ly6C ${ }^{\text {low }}$ monocytes in the tumor vasculature. Potential FAK dependentactivities such as emigration from the vasculature into the tumor bed could be tested. Beyond this application, the ability to observe monocytes by fluorescence would be beneficial in experiments that incorporated flow cytometry or immunofluorescence. This also has applications for competitive adoptive transfer experiments. WTCX $3 \mathrm{CR} 1^{\mathrm{GFP} /+}$ and $\mathrm{FAK}^{\Delta \text { myeloid }} \mathrm{CX} 3 \mathrm{CR} 1^{\mathrm{GFP} /+}$ mice 
could be crossed onto different CD45 allelic variants, which would permit adoptively transferred monocytes to be distinguished by flow cytometry (Carlin et al., 2013).

\subsection{Perspective on FAK and Pyk2 in mononuclear phagocytes}

We remain in the early stages of understanding when FAK and Pyk2 are expressed during mononuclear phagocyte differentiation and maturation and the roles they play in the regulation of cell function. Evidence from this thesis raises a lot of questions about the ability of FAK and Pyk2 to shape the proportion and distribution of monocyte populations, and how they are incorporated into the signaling cascades that permit the MPS to operate effectively in steady state and disease. We now have the ability to selectively isolate monocyte populations, and future studies should be aimed at investigating the functional consequences of FAK and Pyk2 in these cells, while keeping in mind how the expression profile of FAK and Pyk2 in different monocyte subsets could be reflective of a given task that the cell is required to perform. In a broader sense, it is revealing that cells of the monocyte lineage, during their continuum of differentiation, have acquired the requisite tools and checkpoints that permit the formation of distinct cell populations that maintain in some instances a high level of functional plasticity. It remains to be seen how FAK and Pyk2 fit into this scheme. 


\section{Literature Cited}

Ahn, G.O., Brown, J.M. (2008) Matrix metalloproteinase-9 is required for tumor vasculogenesis but not for angiogenesis: role of bone marrow-derived myelomonocytic cells. Cancer Cell $13,193-205$.

Akashi, K., Traver, D., Miyamoto, T., Weissman, I.L. (2000) A clonogenic common myeloid progenitor that gives rise to all myeloid lineages. Nature 404, 193-197.

Allavena, P., Sica, A., Solinas, G., Porta, C., Mantovani, A. (2008) The inflammatory microenvironment in tumor progression: the role of tumor-asociated macrophages. Critical Reviews in Oncology/Hematology 66(1), 1-9.

Almand, B., Clark, J.I., Nikitina, E., van Beynen, J., English, N.R., Knight, S.C., Carbone, D.P., Gabrilovich, D.I. (2001) Increased production of immature myeloid cells in cancer patients: a mechanism of immunosuppression in cancer. The Journal of Immunology 166, 678-689.

Alon, R., Dustin, M.L. (2007) Force as a facilitator of integrin conformational changes during leukocyte arrest on blood vessels and antigen-presenting cells. Immunity 26, 17-27.

Auffray, C., Fogg, D., Garfa, M., Elain, G., Join-Lambert, O., Kayal, S., Sarnacki, S., Cumano, A., Lauvau, G., Geissmann, F. (2007) Monitoring of blood vessels and tissues by a population of monocytes with patrolling behavior. Science 317, 666-670.

Auffray, C., Sieweke, M.H., Geissmann, F. (2009) Blood monocytes: development, heterogeneity, and relationship with dendritic cells. Annu. Rev. Immunol. 27, 669-692. 
Augier, S., Ciucci, T., Luci, C., Carle, G.F., Blin-Wakkach, C., Wakkach, A. (2010) Inflammatory blood monocytes contribute to tumor development and represent a privileged target to improve host imunosurveillance. The Journal of Immunology 185, $7165-7173$.

Austyn, J.M., Gordon, S. (1981) F4/80, a monoclonal antibody directed specifically against the mouse macrophage. Eur. J. Immunol. 11, 805-815.

Avraham, S., London, R., Fu, Y., Ota, S., Hiregowdara, D., Li, J., Jiang, S., Pasztor, L.M., White, R.A., Groopman, J.E., Avraham, H. (1995) Identification and characterization of a novel related adhesion focal tyrosine kinase (RAFTK) from megakaryocytes and brain. The Journal of Biological Chemistry 270(46), 27742-27751.

Avraham, H., Park, S.Y., Schinkmann, K., Avraham, S. (2000) RAFTK/Pyk2-mediated cellular signalling. Cellular Signalling 12, 123-133.

Balkwill, F., Mantovani, A. (2001) Inflammation and cancer: back to Virchow? The Lancet 357, 539-545.

Balkwill, F. (2004) Cancer and the chemokine network. Nature Reviews Cancer 4, 540-550.

Barreda, D.R., Hanington, P.C., Belosevic, M. (2004) Regulation of myeloid development and function by colony stimulating factors. Developmental and Comparative Immunology 28 , 509-554.

Beekhuizen, H., van Furth, R. (1993) Monocyte adherence to human vascular endothelium. Journal of Leukocyte Biology 54, 363-378. 
Bingle, L., Brown, N.J., Lewis, C.E. (2002) The role of tumor-associated macrophages in tumor progression: implications for new anticancer therapies. Journal of Pathology 196, 254265.

Braren, R., Hu, H., Kim, Y.H., Beggs, H.E., Reichardt, L.F., Wang, R. (2006) Endothelial FAK is essential for vascular network stability, cell survival, and lamellipodial formation. The Journal of Cell Biology 172(1), 151-162.

Brinson, A.E., Harding, T., Diliberto, P.A., He, Y., Li, X., Hunter, D., Herman, B., Earp, S., Graves, L.M. (1998) Regulation of a calcium-dependent tyrosine kinase in vascular smooth muscle cells by angiotensin II and platelet-derived growth factor. The Journal of Biological Chemistry 273(3), 1711-1718.

Bronte, V., Apolloni, E., Cabrelle, A., Ronca, R., Serafini, P., Zamboni, P., Restifo, N.P., Zanovello, P. (2000) Identification of a CD $11 \mathrm{~b}^{+} / \mathrm{Gr}-1^{+} / \mathrm{CD} 31^{+}$myeloid progenitor capable of activating or suppressing $\mathrm{CD}^{+} \mathrm{T}$ cells. Blood 96(12), 3838-3846.

Cailleau, R., Young, R., Olive, M., Reeves, Jr., W.J. (1974) Breast tumor cell lines from pleural effusions. The Journal of the National Cancer Institute 53(3), 661-674.

Cambien, B., Pomeranz, M., Millet, M.A., Rossi, B., Schmid-Alliana, A. (2001) Signal transduction involved in MCP-1-mediated monocytic transendothelial migration. Blood 97(2), 359-366.

Carlin, L.M., Stamatiades, E.G., Auffray, C., Hanna, R.N., Glover, L., Vizcay-Barrena, G., Hedrick, C.C., Cook, H.T., Diebold, S., Geissmann, F. (2013a) Nr4a1-dependent Ly6C ${ }^{\text {low }}$ monocytes monitor endothelial cells and orchestrate their disposal. Cell 153, 362-375. 
Carlin, L.M., Auffray, C., Geissmann, F. (2013b) Measuring intravascular migration of mouse Ly6 $\mathrm{C}^{\text {low }}$ monocytes in vivo using intravital microscopy. Current Protocols in Immunology, 14.33.1-14.33.16.

Chioda, M., Peranzoni, E., Desantis, G., Papalini, F., Falisi, E., Samantha, S., Mandruzzato, S., Bronte, V. (2011) Myeloid cell diversification and complexity: an old concept with new turns in oncology. Cancer Metastasis Rev. 30, 27-43.

Chomarat, P., Banchereau, J., Davoust, J., Palucka, A.K. (2000) IL-6 switches the differentiation of monocytes from dendritic cells to macrophages. Nature Immunology 1, 510-514.

Chow, A., Brown, B.D., Merad, M. (2011) Studying the mononuclear phagocyte system in the molecular age. Nature Reviews Immunology 11, 788-798.

Clahsen, T., Schaper, F. (2008) Interleukin-6 acts in the fashion of a classical chemokine on monocytic cells by inducing integrin activation, cell adhesion, actin polymerization, chemotaxis, and transmigration. Journal of Leukocyte Biology 84, 1521-1529.

Clausen, B.E., Burkhardt, C., Reith, W., Renkawitz, R., Forster, I. (1999) Conditional gene targeting in macrophages and granulocytes using LysMcre mice. Transgenic Research 8, $265-277$.

Corsi, J.M., Rouer, E., Girault, J.A., Enslen, H. (2006) Organization and post-transcriptional processing of focal adhesion kinase gene. BMC Genomics 7(198), 1-21.

Cortez-Retamozo, V., Etzrodt, M., Newton, A., Rauch, P.J., Chudnovskiy, A., Berger, C., Ryan, R.J.H., Iwamoto, Y., Marinelli, B., Gorbatov, R., Forghani, R., Novobrantseva, T.I., Koteliansky, V., Figueiredo, J.L., Chen, J.W., Anderson, D.G., Nahrendorf, M., Swirski, F.K., Weissleder, R., Pittet, M.J. (2012) Origins of tumor-associated macrophages and neutrophils. PNAS 109(7), 2491-2496. 
Corzo, C.A., Condamine, T., Lu, L., Cotter, M.J., Youn, J.I., Cheng, P., Cho, H.I., Celis, E., Quiceno, D.G., Padhya, T., McCaffrey, T.V., McCaffrey, J.C., Gabrilovich, D.I. (2010) HIF-1 $\alpha$ regulates function and differentiation of myeloid-derived suppressor cells in the tumor microenvironment. Journal of Experimental Medicine 207, 2439-2453.

Czitrom, A.A., Edwards, S., Phillips, R.A., Bosma, M.J., Marrack, P., Kappler, J.W. (1985) The function of antigen-presenting cells in mice with severe combined immunodeficiency. The Journal of Immunology 134, 2276-2280.

Dai, X.M., Ryan, G.R., Hapel, A.J., Dominguez, M.G., Russell, R.G., Kapp, S., Sylvestre, V., Stanley, E.R. (2002) Targeted disruption of the mouse colony-stimulating factor 1 receptor gene results in osteopetrosis, mononuclear phagocyte deficiency, increased primitive progenitor cell frequencies, and reproductive defects. Blood 99(1), 111-120.

Davie, S.A., Maglione, J.E., Manner, C.K., Young, D., Cardiff, R.D., MacLeod, C.L., Ellies, L.G. (2007) Effects of $\mathrm{FVB} / \mathrm{NJ}$ and $\mathrm{C} 57 \mathrm{Bl} / 6 \mathrm{~J}$ strain backgrounds on mammary tumor phenotype in inducible nitric oxide synthase deficient mice. Transgenic Res. 16, 193-201.

DeKoter, R.P., Walsh, J.C., Singh, H. (1998) PU.1 regulates both cytokine-dependent proliferation and differentiation of granulocyte/macrophage progenitors. The EMBO Journal 17(15), 4456-4468.

DeNardo, D.G., Barreto, J.B., Andreu, P., Vasquez, L., Tawfik, D., Kolhatkar, N., Coussens, L.M. (2009) $\mathrm{CD}^{+} \mathrm{T}$ cells regulate pulmonary metastasis of mammary carcinomas by enhancing protumor properties of macrophages. Cancer Cell 16, 91-102.

De Nichilo, M.O., Yamada, K.M. (1996) Integrin $\alpha v \beta 5$-dependent serine phosphorylation of paxillin in cultured human macrophages adherent to vitronectin. The Journal of Biological Chemistry 271(18), 11016-11022. 
De Palma, M., Venneri, M.A., Galli, R., Sergi, L.S., Politi, L.S., Sampaolesi, M., Naldini, L. (2005) Tie2 identifies a hematopoietic lineage of proangiogenic monocytes required for tumor vessel formation and a mesenchymal population of pericyte progenitors. Cancer Cell 8, 211-226.

De Palma, M., Murdoch, C., Venneri, M.A., Naldini, L., Lewis, C.E. (2007) Tie2-exprssing monocytes: regulation of tumor angiogenesis and therapeutic implications. TRENDS in Immunology 28(12), 519-524.

Diaz-Montero, C.M., Salem, M.L., Nishimura, M.I., Garrett-Mayer, E., Cole, D.J., Montero, A.J. (2009) Increased circulating myeloid-derived suppressor cells correlate with clinical cancer stage, metastatic tumor burden, and doxorubicin-cyclophosphamide chemotherapy. Cancer Immunol. Immunother. 58, 49-59.

Doedens, A.L., Stockmann, C., Rubinstein, M.P., Liao, D., Zhang, N., DeNardo, D.G., Coussens, L.M., Karin, M., Goldrath, A.W., Johnson, R.S. (2010) Macrophage expression of hypoxia-inducible factor-1 $\alpha$ suppresses T-cell function and promotes tumor progression. Cancer Research 70(19), 7465-7475.

Dolcetti, L., Peranzoni, E., Ugel, S., Marigo, I., Gomez, A.F., Mesa, C., Geilich, M., Winkels, G., Traggiai, E., Casati, A., Grassi, F., Bronte, V. (2010) Hierarchy of immunosuppressive strength among myeloid-derived suppressor cell subsets is determined by GM-CSF. Eur. J. Immunol. 40, 22-35.

Dorshkind, K., Pollack, S.B., Bosma, M.J., Phillips, R.A. (1985) Natiral killer (NK) cells are present in mice with severe combined immunodeficiency (scid). The Journal of Immunology 134, 3798-3801. 
Dylla, S.J., Deyle, D.R., Theunissen, K., Padurean, A.M., Verfaillie, C.M. (2004) Integrin engagement-induced inhibition of human myelopoiesis is mediated by proline-rich tyrosine kinase 2 gene products. Experimental Hematology 32, 365-374.

Egeblad, M., Werb, Z. (2002) New functions for the matrix metalloproteinases in cancer progression. Nature Reviews Cancer 2, 161-174.

Egeblad, M., Ewald, A., Askautrud, H.A., Truitt, M.L., Welm, B.E., Bainbridge, E., Peeters, G., Krummel, M.F., Werb, Z. (2008) Visualizing stromal cell dynamics in different tumor microenvironments by spinning disk confocal microscopy. Disease Models \& Mechanisms 1, 155-167.

Egeblad, M., Nakasone, E.S., Werb, Z. (2010) Tumors as organs: complex tissues that interface with the entire organism. Dev. Cell. 18(6), 884-901.

Elinav, E., Nowarski, R., Thaiss, C.A., Hu, B., Jin, C., Flavell, R.A. (2013) Inflammationinduced cancer: crosstalk between tumors, immune cells and microorganisms. Nature Reviews Cancer 13, 759-771.

Enver, T., Pera, M., Peterson, C., Andrews, P.W. (2009) Stem cell states, fates, and the rules of attraction. Cell Stem Cell 4, 387-397.

Eulenfeld, R., Dittrich, A., Khouri, C., Muller, P.J., Mutze, B., Wolf, A., Schaper, F. (2012) Interleukin-6 signalling: More than Jaks and STATs. European Journal of Cell Biology 91, 486-495.

Fleetwood, A.J., Lawrence, T., Hamilton, J.A., Cook, A.D. (2007) Granulocyte-macrophage colony-stimulating factor (CSF) and macrophage CSF-dependent macrophage phenotypes display differences in cytokine profiles and transcription factor activities: 
implications for CSF blockade in inflammation. The Journal of Immunology 178, 52455252.

Fogg, D.K., Sibon, C., Miled, C., Jung, S., Aucouturier, P., Littman, D.R., Cumano, A., Geissmann. (2006) A clonogenic bone marrow progenitor specific for macrophages and dendritic cells. Science 311, 83-87.

Francke, A., Herold, J., Weinert, S., Strasser, R.H., Braun-Dullaeus, R.C. (2011) Generation of mature murine monocytes from heterogeneous bone marrow and description of their properties. J. of Histochem. \& Cytochem. 59, 813-825.

Frese, K.K., Tuveson, D.A. (2007) Maximizing mouse cancer models. Nature Reviews Cancer 7, 645-658.

Fridman, R., Toth, M., Chvyrkova, I., Meroueh, S.O., Mobashery, S. (2003) Cell surface association of matrix metalloproteinase-9 (gelatinase B). Cancer and Metastasis Reviews 22, 153-166.

Fujita, M., Takada, Y.K., Takada, Y. (2012) Integrins $\quad \alpha v \beta 3$ and $\alpha 4 \beta 1$ act as coreceptors for fractalkine, and the integrin-binding defective mutant of fractalkine is an antagonist of CX3CR1. The Journal of Immunology 189, 5809-5819.

Gabrilovich, D.I., Nagaraj, S. (2009) Myeloid-derived suppressor cells as regulators of the immune system. Nature Reviews Immunology 9, 162-174.

Gabrilovich, D., Ostrand-Rosenberg, S., Bronte, V. (2012) Coordinated regulation of myeloid cells by tumors. Nature Reviews Immunology 12, 253-268. 
Galli, S.J., Borregaard, N., Wynn, T.A. (2011) Phenotypic and functional plasticity of cells of innate immunity: macrophages, mast cells, and neutrophils. Nature Immunology 12(11), $1035-1044$.

Geissmann, F., Jung, S., Littman, D.R. (2003) Blood monocytes consist of two principal subsets with distinct migratory properties. Immunity 19, 71-82.

Gelman, I.H. (2003) Pyk 2 FAKs, any two FAKs. Cell Biology International 27, 507-510.

Gerszten, R.E., Garcia-Zepeda, E.A., Lim, Y.C., Yoshida, M., Ding, H.A., Gimbrone Jr., M.A., Luster, A.D., Luscinskas, F.W., Rosenzweig, A. (1999) MCP-1 and IL-8 trigger firm adhesion of monocytes to vascular endothelium under flow conditions. Nature 398, 718723.

Ginhoux, F., Greter, M., Leboeuf, M., Nandi, S., See, P., Gokhan, S., Mehler, M.F., Conway, S.J., Ng, L.G., Stanley, E.R., Samokhvalov, I.M., Merad, M. (2010) Fate mapping analysis reveals that adult microglia derive from primitive macrophages. Science 330, $841-845$

Glodek, A.M., Le, Y., Dykxhoorn, D.M., Park, S.Y., Mostoslavsky, G., Mulligan, R., Lieberman, J., Beggs, H.E., Honczarenko, M., Silberstein, L.E. (2007) Focal adhesion kinase is required for CXCL-12-induced chemotactic and pro-adhesive responses in hematopoietic precursor cells. Leukemia 21, 1723-1732.

Goda, S., Imai, T., Yoshie, O., Yoneda, O., Inoue, H., Nagano, Y., Okazaki, T., Imai, H., Bloom, E.T., Domae, N., Umehara, H. (2000) CX3C-chemokine, fractalkine-enhanced adhesion of THP-1 cells to endothelial cells through integrin-dependent and -independent mechanisms. The Journal of Immunology 164, 4313-4320.

Gordon, S. (2003) Alternative activation of macrophages. Nature Reviews Immunology 3, 23-35. 
Gordon, S., Taylor, P.R. (2005) Monocyte and macrophage heterogeneity. Nature Reviews Immunology 5, 953-964.

Gordon, S. (2008) Elie Metchnikoff: Father of natural immunity. Eur. J. Immunol. 38, 32573264.

Gordon, S. (2007) The macrophage: Past, present, and future. European Journal of Immunology, 37, S9-17.

Goswami, S., Sahai, E., Wyckoff, J.B., Cammer, M., Cox, D., Pixley, F.J., Stanley, E.R., Segall, J.E., Condeelis, J.S. (2005) Macrophages promote the invasion of breast carcinoma cells via a colony-stimulating factor-1/epidermal growth factor paracrine loop. Cancer Research 65(12), 5278-5283.

Guy, C.T., Cardiff, R.D., Muller, W.J. (1992) Induction of mammary tumors by expression of polyomavirus middle $\mathrm{T}$ oncogene: a transgenic mouse model for metastatic disease. Molecular and Cellular Biology 12(3), 954-961.

Hamilton, J.A. (2008) Colony-stimulating factors in inflammation and autoimmunity. Nature Reviews Immunology 8, 533-544.

Hamilton, J.A., Achuthan, A. (2013) Colony stimulating factors and myeloid cell biology in health and disease. Trends in Immunology 34(2), 81-89.

Hanna, R.N., Carlin, L.M., Hubbeling, H.G., Nackiewicz, D., Green, A.M., Punt, J.A., Geissmann, F., Hedrick, C.C. (2011) The transcription factor NR4A1 (Nur77) controls bone marrow differentiation and the survival of Ly6 $6 \mathrm{C}^{-}$monocytes. Nature Immunology 12(8), 778-785. 
Hanna, R.N., Shaked, I., Hubbeling, H.G., Punt, J.A., Wu, R., Herrley, E., Zaugg, C., Pei, H., Geissmann, F., Ley, K., Hedrick, C.C. (2012) NR4A1 (Nur77) deletion polarizes macrophages toward an inflammatory phenotype and increases atherosclerosis. Circulation Research 110, 416-427.

Hanninen, A., Jaakkola, I., Salmi, M., Simell, O., Jalkanen, S. (1997) Ly-6C regulates endothelial adhesion and homing of $\mathrm{CD}^{+} \mathrm{T}$ cells by activating integrin-dependent adhesion pathways. PNAS 94, 6898-6903.

Hashimoto, D., Chow, A., Noizat, C., Teo, P., Beasley, Leboeuf, M., Becker, C.D., See, P., Prics, J., Lucas, D., Greter, M., Mortha, A., Boyer, S.W., Forsberg, E.C., Tanaka, M., van Rooijen, N., Garcia-Sastre, A., Stanley, E.R., Ginhoux, F., Frenette, P.S., Merad, M. (2013) Tissue-resident macrophages self-maintain locally throughout adult life with minimal contribution from circulating monocytes. Immunity 38, 792-804.

Herter, J., Zarbock, A. (2013) Integrin regulation during leukocyte recruitment. The Journal of Immunology 190, 4451-4457.

Hettinger, J., Richards, D.M., Hansson, J., Barra, M.M., Joschko, A.C., Krijgsveld, J., Feuerer, M. (2013) Origin of monocytes and macrophages in a committed progenitor. Nature Immunology 14(8), 821-830.

Hildebrand, J.D., Schaller, M.D., Parsons, J.T. (1993) Identification of sequences required for the efficient localization of the focal adhesion kinase, $\mathrm{pp} 125^{\mathrm{FAK}}$, to cellular focal adhesions. The Journal of Cell Biology 123(4), 993-1005. 
Hoeffel, G., Wang, Y., Greter, M., See, P., Teo, P., Malleret, B., Leboeuf, M., Low, D., Oller, G., Almeida, F., Choy, S.H.Y., Grisotto, M., Renia, L., Conway, S.J., Stanley, E.R., Chan, J.K.Y., Ng, L.G., Samokhvalov, I.M., Merad, M., Ginhoux, F. (2012) Adult Langerhans cells derive predominantly from embryonic fetal liver monocytes with a minor contribution of yolk sac-derived macrophages. Journal of Experimental Medicine 209(6), $1167-1181$

Hoffmann, J.A., Kafatos, F.C., Janeway, C.A., Ezekowitz, R.A.B. (1999) Phylogenetic persepctives in innate immunity. Science 284, 1313-1318.

Hotchin, N.A., Hall, A. (1995) The assembly of integrin adhesion complexes requires both extracellular matrix and intracellular rho/rac GTPases. The Journal of Cell Biology 131(6), 1857-1865.

Hume, D.A., Ross, I.L., Himes, S.R., Sasmono, R.T., Wells, C.A., Ravasi, T. (2002) The mononuclear phagocyte system revisited. Journal of Leukocyte Biology 72, 621-627.

Hume, D.A. (2006) The mononuclear phagocyte system. Current Opinion in Immunology 18, 4953.

Hume, D.A. (2008) Differentiation and heterogeneity in the mononuclear phagocyte system. Mucosal Immunology 1(6), 432-441.

Ikebuchi, K., Wong, G.G., Clark, S.C., Ihle, J.N., Hirai, Y., Ogawa, M. (1987) Interleukin 6 enhancement of interleukin 3-dependent proliferation of multipotential hemopoietic progenitors. PNAS 84, 9035-9039.

Ilic, D., Furuta, Y., Kanazawa, S., Takeda, N., Sobue, K., Nakatsuji, N., Nomura, S., Fujimoto, J., Okada, M., Yamamoto, T., Aizawa, S. (1995) Reduced cell motility and enhanced focal adhesion contact formation in cells from FAK-deficient mice. Nature 377, 539-544. 
Imai, T., Hieshima, K., Haskell, C., Baba, M., Nagira, M., Nishimura, M., Kakizaki, M., Takagi, S., Nomiyama, H., Schall,T., Yoshie, O. (1997) Identification and molecular characterization of fractalkine receptor $\mathrm{CX} 3 \mathrm{CR} 1$, which mediates both leukocyte migration and adhesion. Cell 91, 521-530.

Imhof, B.A., Aurrand-Lions, M. (2004) Adhesion mechamisms regulating the migration of monocytes. Nature Reviews Immunology 4, 432-444.

Ingersoll, M.A., Spanbroek, R., Lottaz, C., Gautier, E.L., Frankenberger, M., Hoffmann, R., Lang, R., Haniffa, M., Collin, M., Tacke, F., Habenicht, A.J.R., Ziegler-Heitbrock, L., Randolph, G.J. (2010) Comparison of gene expression profiles between human and mouse monocyte subsets. Blood 115(3), e10-e19.

Iwasaki, H., Akashi, K. (2007) Myeloid lineage commitment from the hematopoietic stem cell. Immunity 26, 726-740.

Jacob, S.S., Sudhakaran, P.R. (2001) Monocyte-macrophage differentiation in three dimensional collagen lattice. Biochimica et Biophysica Acta 1540, 50-58.

Jacob, S.S., Shastry, P., Sudhakaran, P.R. (2002) Monocyte-macrophage differentiation in vitro: modulation by extracellular matrix protein substratum. Molecular and Cellular Biochemistry 233, 9-17.

Jakubzick, C., Gautier, E.L., Gibbings, S.L., Sojka, D.K., Schlitzer, A., Johnson, T.E., Ivanov, S., Duan, Q., Bala, S., Condon, T., van Rooijen, N., Grainger, J.R., Belkaid, Y., Ma'ayan, A., Riches, D.W.H., Yokoyama, W.M., Ginhoux, F., Henson, P.M., Randolph, G.J. (2013) Minimal differentiation of classical monocytes as they survey steady-state tissues and transport antigen to lymph nodes. Immunity 39, 599-610. 
Jessani, N., Humphrey, M., McDonald, W.H., Niessen, S., Masuda, K., Gangadharan, B., Yates III, J.R., Mueller, B.M., Cravatt, B.F. (2004) Carcinoma and stromal enzyme activity profiles associated with breast tumor growth in vivo. PNAS 101(38), 13756-13761.

Jessani, N., Niessen, S., Mueller, B.M., Cravatt, B.F. (2005) Breast cancer cell lines grown in vivo. Cell Cycle 4(2), 253-255.

Jutila, M.A., Kroese, F.G.M., Jutila, K.L., Stall, A.M., Fiering, S., Herzenberg, L.A., Berg, E.L., Butcher, E.C. (1988) Ly-6C is a monocyte/macrophage and endothelial cell differentiation antigen regulated by interferon-gamma. Eur. J. Immunol. 18, 1819-1826.

Kamei, M., Carman, C.V. (2010) New observations on the trafficking and diapedesis of monocytes. Current Opinion in Hematology 17, 43-52.

Kamoshida, G., Matsuda, A., Sekine, W., Mizuno, H., Oku, T., Itoh, S., Irimura, T., Tsuji, T. (2012) Monocyte differentiation induced by co-culture with tumor cells involves RGDdependent cell adhesion to extracellular matrix. Cancer Letters 315(2), 145-152.

Kanner, S.B., Reynolds, A.B., Vines, R.R., Parsons, J.T. (1990) Monoclonal antibodies to individual tyrosine-phosphorylated protein substrates of oncogene-encoded tyrosine kinases. PNAS 87, 3328-3332.

Kaplan, G., Gaudernack, G. (1982) In vitro differentiation of human monocytes. Journal of Experimental Medicine 156, 1101-1114.

Kedzierska, K., Vardaxis, N.J., Jaworowski, A., Crowe, S.M. (2001) FcyR-mediated phagocytosis by human macrophages involves Hck, Syk, and Pyk2 and is augumented by GM-CSF. Journal of Leukocyte Biology 70, 322-328. 
Kessenbrock, K., Plaks, V., Werb, Z. (2010) Matrix metalloproteinases: regulators of the tumor microenvironment. Cell 141, 52-67.

Kharbanda, S., Saleem, A., Yuan, Z., Emoto, Y., Prasad, K.V.S., Kufe, D. (1995) Stimulation of human monocytes with macrophage colony-stimulating factor induces a Grb2-mediated association of the focal adhesion kinase pp $125^{\mathrm{FAK}}$ and dynamin. PNAS 92, 6132-6136.

Kornberg, L., Earp, H.S., Parsons, J.T., Schaller, M., Juliano, R.L. (1992) Cell adhesion or integrin clustering increases phosphorylation of a focal adhesion-associated tyrosine kinase. The Journal of Biological Chemistry 267(33), 23439-23442.

Kume, A., Nishiura, H., Suda, J., Suda, T. (1997) Focal adhesion kinase upregulated by granulocyte-macrophage colony-stimulating factor but not by interleukin-3 in differentiating myeloid cells. Blood 89, 3434-3442.

Kusmartsev, S., Gabrilovich, D.I. (2002) Immature myeloid cells and cancer-associated immune suppression. Cancer Immunol. Immunother. 51, 293-298.

Kusmartsev, S., Gabrilovich, D.I. (2005) STAT1 signaling regulates tumor-associated macrophage-mediated T cell deletion. The Journal of Immunology 174, 4880-4891.

Landsman, L., Varol, C., Jung, S. (2007) Distinct differentiation potential of blood monocyte subsets in the lung. The Journal of Immunology 178, 2000-2007.

Laoui, D., Van Overmeire, E., Movahedi, K., Van den Bossche, J., Schouppe, E., Mommer, C., Nikolaou, A., Morias, Y., De Baetselier, P., Van Ginderachter, J. (2011) Mononuclear phagocyte heterogeneity in cancer: Different subsets and activation states reaching out at the tumor site. Immunobiology 216, 1192-1202. 
Laudanna, C., Kim, J.Y., Constantin, G., Butcher, E. (2002) Rapid leukocyte integrin activation by chemokines. Immunological Reviews 186, 37-46.

Lee, H.W., Choi, H.J., Ha, S.J., Lee, K.T., Kwon, Y.G. (2013) Recruitment of monocytes/macrophages in different tumor microenvironments. Biochimica et Biophysica Acta 1835, 170-179.

Leenen, P.J.M., Melis, M., Slieker, W.A.T., Van Ewijk, W. (1990) Murine macrophage precursor characterization II. Monoclonal antibodies against macrophage precursor antigens. Eur. J. Immunol. 20, 27-34.

Leenen, P.J.M., de Bruijn, M.F.T.R., Voerman, J.S.A., Campbell, P.A., van Ewijk, W. (1994) Markers of mouse macrophage development detected by monoclonal antibodies. J. of Immun. Methods 174, 5-19.

Lenny, N., Westendorf, J.J., Hiebert S.W. (1997) Transcriptional regulation during myelopoiesis. Molecular Biology Reports 24, 157-168.

Lenzo, J.C., Turner, A.L., Cook, A.D., Vlahos, R., Anderson, G.P., Reynolds, E.C., Hamilton, J.A. (2012) Control of macrophage lineage populations by CSF-1 receptor and GM-CSF in homeostasis and inflammation. Immunology and Cell Biology 90, 429-440.

Lev, S., Moreno, H., Martinez, R., Canoll, P., Peles, E., Musacchio, J.M., Plowman, G.D., Rudy, B., Schlessinger, J. (1995) Protein tyrosine kinase PYK2 involved in $\mathrm{Ca}^{+}$-induced regulation of ion channel and MAP kinase functions. Nature 376, 737-745.

Lewis, C.E., Pollard, J.W. (2006) Distinct role of macrophages in different tumor microenvironments. Cancer Research 66(2), 605-612. 
Ley, K., Miller, Y.I., Hedrick, C.C. (2011) Monocyte and macrophage dynamics during atherogenesis. Arterioscler. Thromb. Vasc. Biol. 31, 1506-1516.

Ley, K. (2003) The role of selectins in inflammation and disease. TRENDS in Molecular Medicine 9(6), 263-268.

Li, X., Hunter, D., Moris, J., Haskill, J.S., Earp, H.S. (1998) A calcium-dependent tyrosine kinase splice variant in human monocytes. The Journal of Biological Chemistry 273(16), 93619364.

Lim, S.T., Chen, X.L., Lim, Y., Hanson, D.A., Vo, T.T., Howerton, K., Larocque, N., Fisher, S.J., Schlaepfer, D.D., Ilic, D. (2008) Nuclear FAK promotes cell proliferation and survival through FERM-enhanced p53 degradation. Molecular Cell 29, 9-22.

Lim, S.T., Miller, N.L.G., Nam, J.O., Chen, X.L., Lim, Y., Schlaepfer, D.D. (2010) Pyk2 inhibition of p53 as an adaptive and intrinsic mechanism facilitating cell proliferation and survival. Journal of Biological Chemistry 285(3), 1743-1753.

Lin, E.Y., Nguyen, A.V., Russell, R.G., Pollard, J.W. (2001) Colony-stimulating factor 1 promotes progression of mammary tumors to malignancy. Journal of Experimental Medicine 193(6), 727-739.

Lin, E.Y., Jones, J.G., Li, P., Zhu, L., Whitney, K.D., Muller, W.J., Pollard, J.W. (2003) Progression to malignancy in the polyoma middle $\mathrm{T}$ oncoprotein mouse breast cancer model provides a reliable model for human diseases. American Journal of Pathology 163(5), 2113-2126.

Lin, E.Y., Li, J.F., Gnatovskiy, L., Deng, Y., Zhu, L., Grzesik, D.A., Qian, H., Xue, X., Pollard, J.W. (2006) Macrophages regulate the angiogenic switch in a mouse model of breast cancer. Cancer Research 66(23), 11238-11246. 
Lin, H., Lee, E., Hestir, K., Leo, C., Huang, M., Bosch, E., Halenbeck, R., Wu, G., Zhou, A., Behrens, D., Hollenbaugh, D., Linnemann, T., Qin, M., Wong, J., Chu, K., Doberstein, S.K., Williams, L.T. (2008) Discovery of a cytokine and its receptor by finctional screening of the extracellular proteome. Science 320, 807-811.

Lin, S.L., Castano, A.P., Nowlin, B.T., Lupher, M.L., Duffield, J.S. (2009) Bone marrow Ly6 $\mathrm{C}^{\text {high }}$ monocytes are selectively recruited to injured kidney and differentiate into functionally distinct populations. The Journal of Immunology 183, 6733-6743.

Lin, T.H., Yurochko, A., Kornberg, L., Morris, J. Walker, J.J., Haskill, S. (1994) The role of protein tyrosine phosphorylation in integrin-mediated gene induction in monocytes. The Journal of Cell Biology 126(6), 1585-1593.

Ma, G., Pan, P.Y., Eisenstein, S., Divino, C.M., Lowell, C.A., Takai, T., Chen, S.H. (2011) Paired immunoglobin-like receptor-B regulates the suppressive function and fate of myeloid-derived suppressor cells. Immunity 34, 385-395.

MacDonald, K.P.A., Palmer, J.S., Cronau, S., Seppanen, E., Olver, S., Raffelt, N.C., Kuns, R., Pettit, A.R., Clouston, A., Wainwright, B., Branstetter, D., Smith, J., Paxton, R.J., Cerretti, D.P., Bonham, L., Hill, G.R., Hume, D.A. (2010) An antibody against the colony-stimulating factor 1 receptor depletes the resident subset of monocytes and tissue-and tumor-associated macrophages but does not inhibit inflammation. Blood 116(19), 3955-3963.

Maglione, J.E., Moghanaki, D., Young, L.J.T., Manner, C.K., Ellies, L.G., Joseph, S.O., Nicholson, B., Cardiff, R.D., MacLeod, C.L. (2001) Transgenic polyoma middle-T mice model premaglinant mammary disease. Cancer Research 61, 8298-8305. 
Mantovani, A., Sica, A., Locati, M. (2005) Macrophage polarization comes of age. Immunity 23(4), 344-346.

Mantovani, A., Sica, A., Locati, M. (2007) New vistas on macrophage differentiation and activation. Eur. J. Immunol. 37, 14-16.

Marigo, I., Bosio, E., Solito, S., Mesa, C., Fernandez, A., Dolcetti, L., Ugel, S., Sonda, N., Bicciato, S., Falisi, E., Calabrese, F., Basso, G., Zanovello, P., Cozzi, E., Mandruzzato, S., Bronte, V. (2010) Tumor-induced tolerance and immune suppression depend on the C/EBP $\beta$ Transcription Factor. Immunity 32, 790-802.

Martinez, F.O., Gordon, s., Locati, M., Mantovani, A. (2006) Transcriptional profiling of the human monocyte-to-macrophage differentiation and polarization: New molecules and patterns of gene expression. The Journal of Immunology 177, 7303-7311.

Martinez-Gonzalez, J., Badimon, L. (2005) The NR4A subfamily of nuclear receptors: new early genes regulated by growth factors in vascular cells. Cardiovascular Research 65, 609618.

Mazzon, C., Anselmo, A., Soldani, C., Cibella, J., Ploia, C., Moalli, F., Burden, S.J., Dustin, M.L., Sarukhan, A., Viola, A. (2012) Agrin is required for survival and function of monocytic cells. Blood 119, 5502-5511.

McKercher, S.R., Torbett, B.E., Anderson, K.L., Henkel, G.W., Vestal, D.J., Baribault, H., Klemsz, M., Feeney, A.J., Wu, G.E., Paige, C.J., Maki, R.A. (1996) Targeted disruption of the PU.1 gene results in multiple hematopoietic abnormalities. The EMBO Journal $15(20), 5647-5658$. 
Melani, C., Chiodoni, C., Forni, G., Colombo, M.P. (2003) Myeloid cell expansion elicited by the progression of spontaneous mammary carcinomas in c-erB-2 transgenic BALB/c mice suppresses immune reactivity. Blood 102(6), 2138-2145.

Metcalf, D. (1989) The molecular control of cell division, differentiation commitment and maturation in haemopoietic cells. Nature 339, 27-30.

Miranti, C.K., Brugge, J.S. (2002) Sensing the environment: a historical perspective on integrin signal transduction. Nature Cell Biology 4, 83-90.

Modlin, R.L., Cheng, G. (2004) From plankton to pathogen recognition. Nature Medicine 10(11), $1173-1174$

Mon, N.N., Hasegawa, H., Thant, A.A., Huang, P., Tanimura, Y., Senga, T., Hamaguchi, M. (2006) A role for focal adhesion kinase signaling in tumor necrosis factor- $\alpha$-dependent matrix metalloproteinase-9 production in a cholangiocarcinoma cell line, CCKS1. Cancer Research 66(13), 6778-6784.

Mossadegh-Keller, N., Sarrazin, S., Kandalla, P.K., Espinosa, L., Stanley, E.R., Nutt, S.L., Moore, J., Sieweke, M.H. (2013) M-CSF instructs myeloid lineage fate in single haematopoietic stem cells. Nature 497, 239-244.

Mosser, D.M., Edwards, J.P. (2008) Exploring the full spectrum of macrophage activation. Nature Reviews Immunology 8, 958-969.

Movahedi, K., Guilliams, M., Van den Bossche, J., Van den Bergh, R., Gysemans, C., Beschin, A., De Baetselier, P., Van Ginderachter, J. (2008) Identification of discrete tumorinduced myeloid-derived suppressor cell subpopulations with distinct $\mathrm{T}$ cell-suppressive activity. Blood 111(8), 4233-4244. 
Movahedi, K., Laoui, D., Gysemans, C., Baeten, M., Stange,G., Van den Bossche, J., Mack, M., Pipeleers, D., Veld, P.I., De Baetselier, P., Van Ginderachter, J.A. (2010) Different tumor microenvironments contain functionally distinct subsets of macrophages derived from Ly6C(high) monocytes. Cancer Research 70(14), 5728-5739.

Muller, W.A., Randolph, G.J. (1999) Migration of leukocytes across endothelium and beyond: molecules involved in the transmigration and fate of monocytes. Journal of Leukocyte Biology 66(5), 698-704.

Murdoch, C., Lewis, C.E. (2005) Macrophage migration and gene expression in response to tumor hypoxia. Int. J. Cancer 117, 701-708.

Murray, P.J., Wynn, T.A. (2011) Protective and pathogenic functions of macrophage subsets. Nature Reviews Immunology 11, 723-737.

Nahrendorf, M., Swirski, F.K., Aikawa, E., Stangenberg, L., Wurdinger, T., Figueiredo, J.L., Libby, P., Weissleder, R., Pittet, M.J. (2007) The healing myocardium sequentially mobilizes two monocyte subsets with divergent and complementary functions. Journal of Experimental Medicine 204, 3037-3047.

Nahrendorf, M., Swirski, F.K. (2013) Monocyte and macrophage heterogeneity in the heart. Circ. Res. 112, 1624-1633.

Nerlov, C., Graf, T. (1998) PU.1 induces myeloid lineage commitment in multipotent hematopoietic progenitors. Genes \& Development 12, 2403-2412.

Nixon-Fulton, J.L., Witte, P.L., Tigelaar, R.E., Bergstresser, P.R., Kumar, V. (1987) Lack of dendritic Thy $-1^{+}$epidermal cells in mice with severe combined immunodeficiency disease. The Journal of Immunology 138, 2902-2905. 
Noel, A., Jost, M., Maquoi, E. (2008) Matrix metalloproteinases at cancer tumor-host interface. Seminars in Cell \& Developmental Biology 19, 52-60.

Novosyadlyy, R., Vijayakumar, A., Lann, D., Fierz, Y., Kurshan, N., LeRoith. (2009) Physical and functional interaction between polyoma virus middle $\mathrm{T}$ antigen and insulin and IGFI receptors is required for oncogene activation and tumor initiation. Oncogene 28(39), $3477-3486$.

Ojalvo, L.S., King, W., Cox, D., Pollard, J.W. (2009) High-density gene expression analysis of tumor-associated macrophages from mouse mammary tumors. Am. J. Pathol. 174(3), $1048-1064$.

Okigaki, M., Davis, C., Falasca, M., Harroch, S., Felsenfeld, D.P., Sheetz, M.P., Schlessinger, J. (2003) Pyk2 reglates multiple signaling events crucial for macrophage morphology and migration. PNAS 100, 10740-10745.

Orkin, S.H., Zon, L.I. (2008) Hematopoiesis: An evolving paradigm for stem cell biology. Cell $132,631-644$.

Owen, K., Pixley, F.J., Thomas, K.S., Vicente-Manzanares, M., Ray, B.J., Horwitz, A.F., Parsons, J.T., Beggs, H.E., Stanley, E.R., Bouton, A.H. (2007) Regulation of lamellipodial persistence, adhesion turnover, and motility in macrophages by focal adhesion kinase. The Journal of Cell Biology 179, 1275-1287.

Pahl, H.L., Scheibe, R.J., Zhang, D.E., Chen, H.M., Galson, D.L., Maki, R.A., Tenen, D.G. (1993) The proto-oncogene PU.1 regulates expression of the myeloid-specific CD11b promoter. The Journal of Biological Chemistry 268(7), 5014-5020. 
Park, M.H., Park, S.Y., Kim, Y. (2008) Induction of proline-rich tyrosine knase2 (Pyk2) through $\mathrm{C} / \mathrm{EBP} \beta$ is involved in PMA-induced monocyte differentiation. FEBS Letters 582, 415 422.

Parsons, J.T., Martin, K.H., Slack, J.K., Taylor, J.M., Weed, S.A. (2000) Focal adhesion kinase: a regulator of focal adhesion dynamics and cell movement. Oncogene 19, 5606-5613.

Parsons, J.T. (2003) Focal adhesion kinase: the first ten years. Journal of Cell Science 116, 14091416.

Pastor-Pareja, J.C., Wu, M., Xu, T. (2008) An innate immune response of blood cells to tumors and tissue damage in Drosophila. Disease Models \& Mechanisms 1, 144-154.

Pederson, L., Winding, B., Foged, N.T., Spelsberg, T.C., Oursler, M.J. (1999) Identification of breast cancer cell line-derived paracrine factors that stimulate osteoclast activity. Cancer Research 59, 5849-5855.

Phillips, R.A., Jewett, M.A., Gaille, B.L. (1989) Growth of human tumors in immune-deficient scid mice and nude mice. Curr. Top. Microbiol. Immunol. 152, 259-263.

Pollard, J.W. (2004) Tumor-educated macrophages promote tumor progression and metastasis. Nature Reviews Cancer 4, 71-78.

Pollard, J.W. (2008) Macrophages define the invasive microenvironment in breast cancer. Journal of Leukocyte Biology 84(3), 623-630.

Pollard, J.W. (2009) Trophic macrophages in development and disease. Nature Reviews Immunology 9, 259-270.

Pommier, A., Audemard, A., Durand, A., Lengagne, R., Delpoux, A., Martin, B., Douguet, L., Le Campion, A., Kato, M., Avril, M.F., Auffray, C., Lucas, B., Prevost-Blondel, A. (2013) 
Inflammatory monocytes are potent antitumor effectors controlled by regulatory $\mathrm{CD} 4^{+} \mathrm{T}$ cells. PNAS 110(32), 13085-13090.

Porcheray, F., Viaud, S., Rimaniol, A.C., Leone, C., Samah, B., Dereuddre-Bosquet, N., Dormont, D., Gras, G. (2005) Macrophage activation switching: an asset for the resolution of inflammation. Clincal and Experimental Immunology 142, 481-489.

Price, J.E., Polyzos, A., Zhang, R.D., Daniels, L.M. (1990) Tumorigenicity and metastasis of human breast carcinoma cell lines in nude mice. Cancer Research 50, 717-721.

Pucci, F., Venneri, M.A., Biziato, D., Nonis, A., Moi, D., Sica, A., Di Serio, C., Naldani, L., De Palma, M. (2009) A distinguishing gene signature shared by tumor-infiltrating Tie2expressing monocytes, blood "resident" monocytes, and embryonic macrophages suggests common functions and developmental relationships. Blood 114(4), 901-914.

Qian, B.Z., Pollard, J.W. (2010) Macrophage diversity enhances tumor progression and metastasis. Cell 141, 39-51.

Robert, J. (2010) Comparative study of tumorigenesis and tumor immunity in invertebrates and nonmammalian vertebrates. Dev. Comp. Immunol. 34(9), 915-925.

Robinson, B.D., Sica, G.L., Liu, Y.F., Rohan, T.E., Gertler, F.B., Condeelis, J.S., Jones, J.G. (2009) Tumor microenvironment of metastasis in human breast carcinoma: a potential prognostic marker linked to hematogenous dissemination. Clinical Cancer Research $15(7), 2433-2441$.

Rosmarin, A.G., Weil, S.C., Rosner, G.L., Griffin, J.D., Amaout, M.A., Tenen, D.G. (1989) Differential expression of $\mathrm{CD} 11 \mathrm{~b} / \mathrm{CD} 18(\mathrm{Mo} 1)$ and myeloperoxidase genes during monocyte differentiation. Blood 73(1), 131-136. 
Sanford, D.E., Belt, B.A., Panni, R.Z., Mayer, A., Deshpande, A.D., Carpenter, D., Mitchem, J.B., Plambeck-Suess, S.M., Worley, L.A., Goetz, B.D., Wang-Gillam, A., Eberlein, T.J., Denardo, D.G., Goedegebuure, S.P., Linehan, D.C. (2013) Inflammatory monocyte mobilization decreases patient survival inpancreatic cancer: a role for targeting the CCL2/CCR2 axis. Clin. Cancer Res. 19(13), 3404-3415.

Sasaki, H., Nagura, K., Ishino, M., Tobioka, H., Kotani, K., Sasaki, T. (1995) Cloning and characterization of cell adhesion kinase beta, a novel protein-tyrosine kinase of the focal adhesion kinase subfamily. The Journal of Biological Chemistry 270(21), 21206-21219.

Sasmono, R.T., Oceandy, D., Pollard, J.W., Tong, W., Pavil, P., Wainwright, B.J., Ostrowski, M.C., Himes, S.R., Hume, D.A. (2003) A macrophage colony-stimulating factor receptor-green fluorescent protein transgene is expressed throughput the mononuclear phagocyte system of the mouse. Blood 101(3), 1155-1163.

Sasmono, R.T., Ehrnsperger, A., Cronau, S.L., Ravasi, T., Kandane, R., Hickey, M.J., Cook, A.D., Himes, S.R., Hamilton, J.A., Hume, D.A. (2007) Mouse neutrophilic granulocytes express mRNA encoding the macrophage colony-stimulating factor receptor (CSF-1R) as well as many other macrophage-specific transcripts and can transdifferentiate into macrophages in vitro in response to CSF-1. Journal of Leukocyte Biology 82, 111-123.

Schaller, M.D., Borgman, C.A., Cobb, B.S., Vines, R.R., Reynolds, A.B., Parsons, J.T. (1992) pp $125^{\mathrm{FAK}}$, a structurally distinctive protein-tyrosine kinase associated with focal adhesions. PNAS 89, 5192-5196.

Schaller, M.D., Hildebrand, J.D., Shannon, J.D., Fox, J.W., Vines, R.R., Parsons, J.T. (1994) Autophosphorylation of the focal adhesion kinase, pp125 $5^{\mathrm{FAK}}$, directs $\mathrm{SH} 2$-dependent binding of pp60 $0^{\mathrm{src}}$. Molecular and Cellular Biology 14(3), 1680-1688. 
Schaller, M.D. (2010) Cellular functions of FAK kinases: insight into molecular mechanisms and novel functions. Journal of Cell Science 123, 1007-1013.

Schenkel, A.R., Mamdouh, Z., Muller, W.A. (2004) Locomotion of monocytes on endothelium is a critical step during extravasation. Nature Immunology 5(4), 393-400.

Schulz, C., Perdiguero, E.G., Chorro, L., Szabo-Rogers, H., Cagnard, N., Kierdorf, K., Prinz, M., Wu, B., Jacobsen, S.E.W., Pollard, J.W., Frampton, J., Liu, K., Geissmann, F. (2012) A lineage of myeloid cells independent of Myb and hematopoietic stem cells. Science 336, $86-90$.

Segarra, M., Vilardell, C., Matsumoto, K., Esparza, J., Lozano, E., Serra-Pages, C., UrbanoMarquez, A., Yamada, K.M., Cid, M.C. (2005) Dual function of focal adhesion kinase in regulating integrin-induced MMP-2 and MMP-9 release by human T lymphoid cells. The FASEB Journal 19, 1875-1877.

Serbina, N.V., Pamer, E.G. (2006) Monocyte emigration from bone marrow during bacterial infection requires signals mediated by chemokine receptor CCR2. Nature Immunology 7(3), 311-317.

Shen, T.L., Park, A.Y.J., Alcaraz, A., Peng, X., Jang, I., Koni, P., Flavell, R.A., Gu, H., Guan, J.L. (2005) Conditional knockout of focal adhesion kinase in endothelial cells reveals its role in angiogenesis and vascular development in late embryogenesis. The Journal of Cell Biology 169(6), 941-952.

Shi, C., Simon, D.I. (2006) Integrin signals, transcription factors, and monocyte differentiation. Trends Cardiovasc Med 16(5), 146-152.

Shi, C., Sakuma, M., Mooroka, T., Liscoe, A., Gao, H., Croce, K.J., Sharma, A., Kaplan, D., Greaves, D.R., Wang, Y., Simon, D.I. (2008) Down-regulation of the forkhead 
transcription factor Foxpl is required for monocyte differentiation and macrophage function. Blood 112(12), 4699-4711.

Shi, C., Pamer, E.G. (2011) Monocyte recruitment during infection and inflammation. Nature Reviews Immunology 11, 762-774.

Shiao, S.L., Ganesan, A.P., Rugo, H.S., Coussens, L.M. (2011) Immune microenvironments in solid tumors: new targets for therapy. Genes \& Development 25, 2559-2572.

Shibata, K., Kikkawa, F., Nawa, A., Thant, A.A., Naruse, K., Mizutani, S., Hamaguchi, M. (1998) Both focal adhesion kinase and c-ras are required for the enhanced matrix metalloproteinase 9 secretion by fibronectin in ovarian cancer cells. Cancer Research $58,900-903$.

Sica, A., Bronte, V. (2007) Altered macrophage differentiation and immune dysfunction in tumor development. The Journal of Clinical Investigation 117(5), 1155-1166.

Sidani, M., Wyckoff, J., Xue, C., Segall, J.E., Condeelis, J. (2006) Probing the microenvironment of mammary tumors using multiphoton microscopy. J Mammary Gland Biol Neoplasia $11,151-163$.

Sieg, D.J., Ilic, D., Jones, K.C., Damsky, C.H., Hunter, T., Schlaepfer, D.D. (1998) Pyk2 and Srcfamily protein-tyrosine kinases compensate for the loss of FAK in fibronectinstimulated signaling events but Pyk2 does not fully function to enhance FAK- cell migration. The EMBO Journal 17(20), 5933-5947.

Sieg, D.J., Hauck, C.R., Illic, D., Klingbeil, C.K., Schaefer, E., Damsky, C.H., Schlaepfer, D.D. (2000) FAK integrates growth-factor and integrin signals to promote cell migration. Nature Cell Biology 2, 249-256. 
Spertini, O., Cordey, A.S., Monai, N., Giuffre, L., Schapira, M. (1996) P-selectin glycoprotein ligand 1 is a ligand for L-selectin on neutrophils, monocytes, and $\mathrm{CD} 34^{+}$hematopoietic progenitor cells. The Journal of Cell Biology 135(2), 523-531.

Stockmann, C., Doedens, A., Weidemann, A., Zhang, N., Takeda,N., Greenberg, J.I., Cheresh, D.A., Johnson, R.S. (2008) Deletion of vascular endothelial growth factor in myeloid cells accelerates tumorigenesis. Nature 456(7223), 814-818.

Stout, R.D., Suttles, J. (2004) Functional plasticity of macrophages: reversible adaptation to changing microenvironments. Journal of Leukocyte Biology 76, 509-513.

Stout, R.D., Jiang, C., Matta, B., Tietzel, I., Watkins, S.K., Suttles, J. (2005) Macrophages sequentially change their functional phenotype in response to changes in microenvironmental influences. The Journal of Immunology 175, 342-349.

Sudhakaran, P.R., Radhika, A., Jacob, S.S. (2007) Monocyte macrophage differentiation in vitro: fibronectin-dependent upregulation of certain macrophage-specific activities. Glycoconj. J. 24, 49-55.

Sunderkotter, C., Nikolic, T., Dillon, M.J., van Rooijen, N., Stehling, M., Drevets, D.A., Leenen, P.J.M. (2004) Subpopulations of mouse blood monocytes differ in maturation stage and inflammatory response. The Journal of Immunology 172, 4410-4417.

Swirski, F.K., Nahrendorf, M., Etzrodt, M., Wildgruber, M., Cortez-Retamozo, V., Panizzi, P., Figueiredo, J.L., Kohler, R.H., Chudnovskiy, A., Waterman, P., Aikawa, E., Mempel, T.R., Libby, P., Weissleder, R., Pittet, M.J. (2009) Identification of splenic reservoir monocytes and their deployment to inflammatory sites. Science 325, 612-616.

Tacke, F., Randolph, G.J. (2006) Migratory fate and differentiation of blood monocyte subsets. Immunobiology 211, 609-618. 
Takahashi, K. (2000) Development and differentiation of macrophages and related cells: Historical review and current concepts. Journal of Clinical and Experimental Hematopathology 41(1), 1-33.

Taylor, P.R., Martinez-Pomares, L., Stacey, M., Lin, H.H., Brown, G.D., Gordon, S. (2005) Macrophage receptors and immune recognition. Annu. Rev. Immunol. 23, 901-944.

Tlsty, T.D., Coussens, L.M. (2005) Tumor stroma and regulation of cancer development. Annu. Rev. Pathol. Mech. Dis. 1, 119-150.

Valledor, A.F., Borras, F.E., Cullell-Young, M., Celada, A. (1998) Transcription factors that regulate monocyte/macrophage differentiation. Journal of Leukocyte Biology 63, 405417.

Van Furth, R., Cohn, Z.A. (1968) The origin and kinetics of mononuclear phagocytes. Journal of Experimental Medicine 128(3), 415-435.

Van Ginderachter, J.A., Meerschaut, S., Liu, Y., Brys, L., De Groeve, K., Ghassabeh, G.H., Raes, G., De Baetselier, P. (2006) Peroxisome proliferator-activated receptor $\gamma$ (PPAR $\gamma$ ) ligands reverse CTL suppression by alternatively activated (M2) macrophages in cancer. Blood 108(2), 525-535.

Varol, C., Yona, S., Jung, S. (2009) Origins and tissue-context-dpendent fates of blood monocytes. Immunology and Cell Biology 87, 30-38.

Vemula, S., Ramdas, B., Hanneman, P., Martin, J., Beggs, H., Kapur, R. (2010) Essential role for focal adhesion kinase in regulating stress hematopoiesis. Blood 116(20), 4103-4115. 
Weber, C., Alon, R., Moser, B., Springer, T.A. (1996) Sequential regulation of $\alpha 4 \beta 1$ and $\alpha 5 \beta 1$ integrin avidity by $\mathrm{CC}$ chemokines in monocytes: implications for transendothelial chemotaxis. The Journal of Cell Biology 134(4), 1063-1073.

Weber, C., Springer, T.A. (1998) Interaction of very late antigen-4 with VCAM-1 supports transendothelial chemotaxis of monocytes by facilitating lateral migration. The Journal of Immunology 161, 6825-6834.

Weis, S.M., Lim, S.T., Lutu-Fuga, K.M., Barnes, L.A., Chen, X.L., Gothert, J.R., Shen, T.L., Guan, J.L., Schlaepfer, D.D., Cheresh, D.A. (2008) Compensatory role for Pyk2 during angiogenesis in adult mice lacking endothelial cell FAK. The Journal of Cell Biology 181(1), 43-50.

Wiktor-Jedrzejczak, W., Bartocci, A., Ferrante Jr., A.W., Ahmed-Ansari, A., Sell, K.W., Pollard, J.W., Stanley, E.R. (1990) Total absence of colony-stimulating factor 1 in the macrophage-deficient osteopetrotic (op/op) mouse. PNAS 87, 4828-4832.

Wojciak-Stothard, B., Williams, L., Ridley, A. (1999) Monocyte adhesion and spreading on human endothelial cells is dependent on Rho-regulated receptor clustering. The Journal of Cell Biology 145(6), 1293-1307.

Wyckoff, J.B., Wang, Y., Lin, E., Li, J., Goswami, S., Stanley, E.R., Segall, J.E., Pollard, J.W., Condeelis, J. (2007) Direct visualization of macrophage-assisted tumor cell intravasation in mammary tumors. Cancer Research 67(6), 2649-2656.

Wynn, T.A., Chawla, A., Pollard, J.W. (2013) Macrophage biology in development, homeostasis and disease. Nature 496, 445-455. 
Xu, H., Manivannan, A., Crane, I., Dawson, R., Liversidge, J. (2008) Critical but divergent roles for CD62L and CD44 in directing blood monocyte trafficking in vivo during inflammation. Blood 112(4), 1166-1174.

Ye, M., Iwasaki, H., Laiosa, C.V., Stadtfeld, M., Xie, H., Heck, S., Clausen, B., Akashi, K., Graf, T. (2003) Hematopoietic stem cells expressing the myeloid lysozyme gene retain longterm, multilineage repopulation potential. Immunity 19, 689-699.

Yona, S., Jung, S. (2010) Monocytes: subsets, origins, fates and functions. Current Opinion in Hematology 17(1), 53-59.

Yona, S., Kim, K.W., Wolf, Y., Mildner, A., Varol, D., Breker, M., Strauss-Ayali, D., Viukov, S., Guilliams, M., Misharin, A., Hume, D.A., Perlman, H., Malissen, B., Zelzer, E., Jung, S. (2013) Fate mapping reveals origins and dynamics of monocytes and tissue macrophages under homeostasis. Immunity 38, 79-91.

Youn, J.I., Nagaraj, S., Collazo, M., Gabrilovich, D.I. (2008) Subsets of myeloid-derived suppressor cells in tumor-bering mice. The Journal of Immunology 181, 5791-5802.

Youn, J.I., Gabrilovich, D.I. (2010) The biology of myeloid-derived suppressor cells: the blessing and the curse of morphological heterogeneity. Eur. J. Immunol. 40, 2969-2975.

Yu, Y., Ross, S.A., Halseth, A.E., Hollenbach, P.W., Hill, R.J., Gulve, E.A., Bond, B.R. (2005) Role of Pyk2 in the development of obesity and insulin resistance. Biochem. and Biophys. Res. Comm. 334, 1085-1091.

Zheng, C., Xing, Z., Bian, C., Guo, C., Akbay, A., Warner, L., Guan, J.L. (1998) Differential regulation of Pyk2 and focal adhesion kinase (FAK). The Journal of Biological Chemistry 273(4), 2384-2389. 
Zigmond, E., Varol, C., Farache, J., Elmaliah, E., Satpathy, A.T., Friedlander, G., Mack, M., Shpigel, N., Boneca, I.G., Murphy, K.M., Shakhar, G., Halpern,Z., Jung, S. (2012) Ly $6 \mathrm{C}^{\text {hi }}$ monocytes in the inflamed colon give rise to proinflammatory effector cells and migratory antigen-presenting cells. Immunity 37, 1076-1090.

Zigmond, E., Jung, S. (2013) Intestinal macrophages: well educated exceptions from the rule. Trends in Immunology 34(4), 162-168. 